
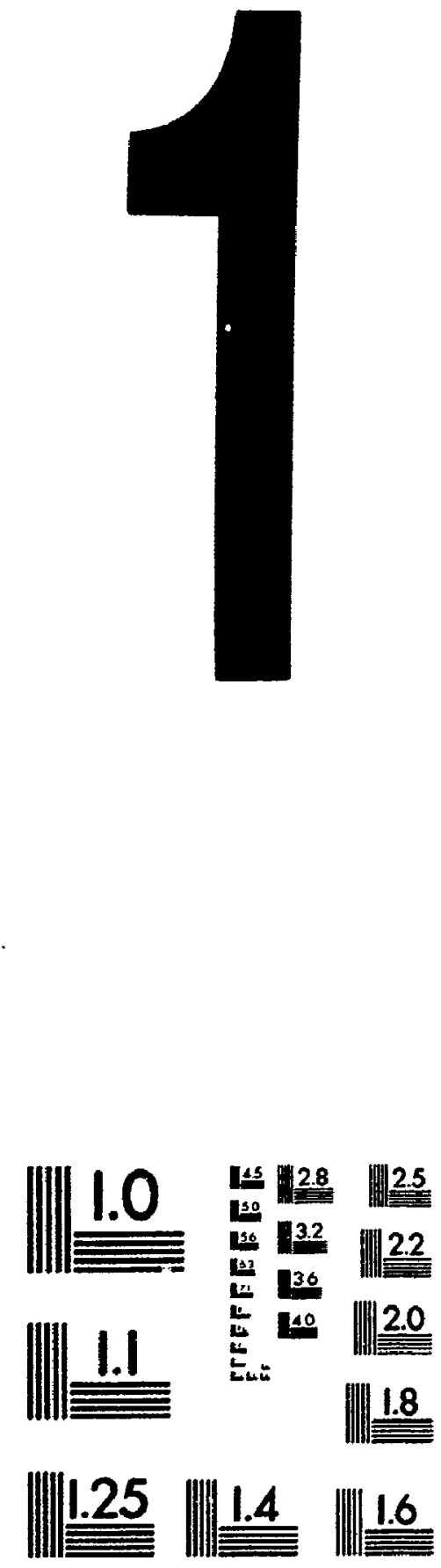

MICROCOPY RESOLUTION TEST CHART

NATIONAL BUREAU OF STANDARDS

STANDARD REFERENCE MATERIAL 1010a

(ANSI and ISO TEST CHART No. 2) 
Bibliothèque nationale

of Canada

du Canada

Canadian Theses Service Service des thèses canadiennes

Ottawa, Canada

KIA ON4

\begin{abstract}
NOTICE
The quality of this microform is heavily dependent upon the quality of the original thesis submitted for microfilming. Every effort has been made to ensure the highest quality of reproduction possible.
\end{abstract}

It pages are missing, contact the university which granted the degree.

Some pages may have indistinct print especially if the original pages were typed with a poor typewriter ribbon or if the university sent us an inferior pholocopy.

Reproduction in full or in pan of this microform is governed by the Canadian Copyright ACt, R.S.C. 1970, c. C-30, and subsequent amendments.
AVIS

La nualité de cette microforme dépend grandement de la qualité de la thèse soumise au microtilmage. Nous avons tout fait pour assurer une qualité supérieure de reproduction.

S'il manque des pages, veuillez communiquer avec l'université qui a conféré le grade.

La qualité d'impression de certaines pages peut laissẹ aे désirer, surtout si les pages originales ont élé daclylogra phiées à l'aide d'un ruban usé ou si l'université nous a lat parvenir une photocopie de qualité intérieure.

La reproduction, même partielle, de celte microforme est soumise à la Loi canadienne sur le droit d'auteur, SRC 1970, c. C-30, ef ses amendements subséquents. 


\title{
FREQUENCY STABILITY CHARACTERIZATION OF HOPPING SOURCES AND MICROPROCESSOR-CONTROLLED FREQUENCY SYNTHESIS
}

by

Grigorios A. Kalivas, Dipl.Eng., M.Eng.

\author{
A thesis submitted to the \\ Faculty of Graduate Studies and Research \\ in partial fulfilment of the requirements \\ for the degree of \\ Doctor of Philosophy
}

Ottawa-Carleton Institute for Electrical Engineering

Faculty of Engineering

Department of Electronics

Carleton University

May 1990

(C)G.A. Kalivas, 1990 
Ottawa. Canada

KIA ON4

\section{NOTICE}

Thequality of this microform is heavily dependent upon the quality of the original thesis submitted for microfilming. Every effort has been made to ensure the highest quality of reproduction possible.

\&f pages are missing. contact the university which granted the degree.

Some pages may have indistinct print especially if the original pages were typed with a poor typewriter ribbon or if the university sent us an inferior photocopy.

Reproduction in full or in pant of this microform is governed by the Canadian Copyright Act. R.S.C. 1970. C.C-30, and subsequent amendments.

\begin{abstract}
AVIS
La qualite de celte microforme dépend grandement de la qualite de la these soumise au microfilmage. Nous avons tout fait pour assurer une qualité supérieure de reproduction.

Sil manque des pages, veuillez communiquer avec tuniversité qui a contéré le grade.

La qualite d'impression de certaines pages peut laisser à désirer. surtout si les pages originates ont été dadylogra. phiées à raide d'un nuban usé ou si funiversité nous a fait parvenir une photocopie de qualité inférieure.
\end{abstract}

La reproduction, méme partielle, de cette microforme est soumise à la Loi canadienne sur le droit dauteur. SRC 1970. c. C-30, et ses amendements subséquents. 
The undersigned hereby recommend to

the Faculty of Graduate Studies and Research

acceptance of the thesis,

FREQUENCY STABILITY CHARACTERIZATION OF HOPPING

SOURCES AND MICROPROCESSOR-CONTROLLED

FREQUENCY SYNTHESIS

submitted by Grigorios Athanasios Kalivas,

in partial fulfilment of the requirements for the degree of

Doctor of Philosophy

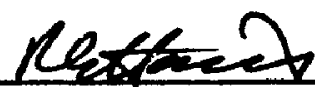

Thesis Supervisor
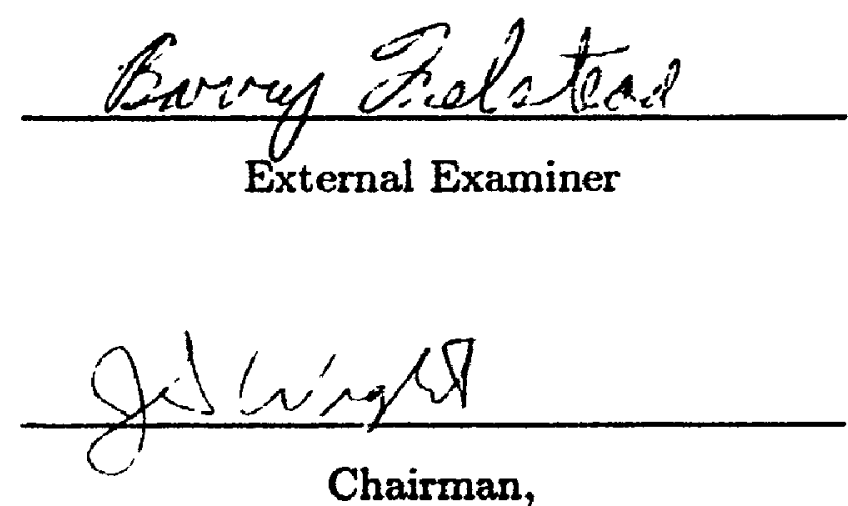

Department of Electronics

Faculty of Engineering

Department of Electronics

Carleton University

June, 1990 


\section{ABSTRACT}

The continuing evolution of microprocessors, digital signal processors and high frequency counters increases the importance of frequency stability characterization of oscillators in the time domain. Although research on the subject has been active for the last 20 years, new time domain (TD) measures continue to evolve. Furthermore, the increasing application of frequency-agile sources for obtaining pulsed signals such as frequency-hopping and radar signals requires the use of this type of stability characterization for fast switching frequency sources.

This thesis is a study of the TD stability of frequency-agile sources such as VCOs under frequency-hopping conditions. A procedure leading to short-term noise characterization is presented and is followed by a novel approach for obtaining expressions for stability measures. Because of its generality, this approach can treat all types of noise (flicker, random walk, etc.) in the same way. Unlike earlier methods, no knowledge of the autocorrelation function of the phase noise is required. As an application of the stability measurement procedure a frequency-synthesis method incorporating these ideas is presented. The corresponding system is a test system and therefore it does not constitute a full scale state of the art synthesizer design. However, it embodies the recent trends towards computer-control and digital processing in frequency synthesis. The synthesizer is operated in a closed-loop mode in which a frequency counter detects departures of the frequency from the desired value and correction is done under computer control. Time domain adaptive algorithms are used in this control procedure. They include a novel modified LMS algorithm which exhibits faster convergence than the simple LMS algorithm. This system was built and tested. Experiment shows that regardless of the number of frequencies or the frequency-hopping pattern, there always exists a flat horizontal portion on the Barues-Allan variance estimator curves for values of averaging intervals in the region of $100 \mu \mathrm{sec}$. The implemented LMS, adaptive algorithm was shown to work as theoretically expected. It is shown that after a cumulative frequency offset of 
$2 \mathrm{MHz}$, the system adjusts its output such that it returns to the expected value within approximately $40 \mathrm{KHz}$. 


\section{ACKNOWLEDGEMENTS}

I would like to express my deepest appreciation to my thesis supervisor Professor Robert G. Harrison, for the continuous guidance, support and encouragement throughout this work. I also wish to thank Professor Mohammed El-Tanany for his interest and helpful discussions. The graduate students and staff of the Department of Electronics also deserve credit for creating a friendly atmosphere where research can be done under conditions suited to an academic institution.

Special thanks belong to the Hardware Manager, Nagui Mikhail, who provided substantial help in practical problems appearing throughout the actual implementation of the system. Diane Dodds deserves special credit for the fast and efficient typing of this thesis. I would like also to thank Heather Roberts for helping me and actually drawing some of the most complicated figures.

I thank also the Department of Electronics as well as the Faculty of Graduate Studies and Research for providing me with financial assistance throughout this work.

I am deeply indebted to my parents, Athanasios and Stella, for their emotional support and financial assistance.

Finally, I would like to thank all my good friends (most of them fellow scientists und engineers) present and gone, for sharing the same passion of getting involved in the front line of knowledge regardless of the diversity of the fields under investigation. 
This thesis is dedicated to

M.A. 


\section{Contents}

ABSTRACT $\ldots \ldots \ldots \ldots \ldots \ldots \ldots \ldots \ldots \ldots \ldots \ldots \ldots$

ACKNOWLEDGEMENTS $\ldots \ldots \ldots \ldots \ldots \ldots \ldots$

DEDICATION $\ldots \ldots \ldots \ldots \ldots \ldots \ldots \ldots \ldots \ldots \ldots$

TABLE OF CONTENTS $\ldots \ldots \ldots \ldots \ldots \ldots$ vii

LIST OF FIGURES $\ldots \ldots \ldots \ldots \ldots \ldots \ldots \ldots \ldots \ldots$ xiii

LIST OF TABLES $\ldots \ldots \ldots \ldots \ldots \ldots \ldots \ldots \ldots \ldots \ldots$

LIST OF SYMBOLS $\ldots \ldots \ldots \ldots \ldots \ldots \ldots \ldots \ldots \ldots$

1 STABILITY GH' OSCILLATORS AND FREQUENCY SYNTHESIS

1.1 INTRODUCTION $\ldots \ldots \ldots \ldots \ldots \ldots \ldots \ldots \ldots$

1.2 FREQUENCY STABILITY OF OSCILLATORS . . . . . . . . . 3

1.3 FREQUENCY SYNTHESIS TECHNIQUES AND HOPPING SYNTHESIZERS $\ldots \ldots \ldots \ldots \ldots \ldots \ldots \ldots$

1.3.1 Iterated Direct Synthesis $\ldots \ldots \ldots \ldots \ldots \ldots$ 
1.3.2 Indirect Phase-Locked Synthesis $\ldots \ldots \ldots \ldots \ldots$

1.3.3 All-Digital Frequency Synthesizers . . . . . . . . . 15

1.3 .4 Hybrid Systems $\ldots \ldots \ldots \ldots \ldots \ldots \ldots$

1.4 APPLICATION OF HOPPING SOURCES IN SPREAD SPECTRUM COMMUNICATION .................. 18

1.4 .1 Definition ..................... 18

1.4.2 Basic Spreading Techniques and Applications . . . . . . 20

1.5 FREQUENCY SYNTHESIS BY A MICROPROCESSOR-CONTROLLED VCO . . . . . . . . . . . . . . . . . . . 26

2 TIME DOMAIN CHARACTERIZATION OF THE SHORT-TERM FREQUENCY STABILITY OF MICROWAVE VCO'S WITH APPLICATION TO HOPPING SOURCES

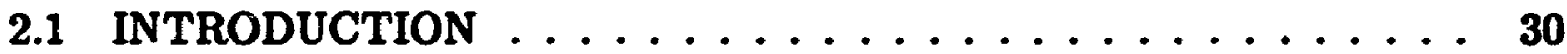

2.2 VARIANCE DEFINITION AND IMPORTANCE, AND THE ROLE OF THE COUNTER IN ITS MEASUREMENT . . . . . . . . . 32

2.3 VARIANCE ESTMMATES AND THEIR EQUIVALENT FILTER REPRESENTATION .................... 38

2.3.1 Barnes-Allan Variance . . . . . . . . . . . . . . 39

2.3.2 Boileau-Picinbono Variance and Generalization of Variance Estimators .....................443

2.3.3 Criterion for the Choice of a Suitable Variance Estimator . . 46 
2.4 A NOVEL APPROACH TO DERIVING CLOSED-FORM EXPRESSIONS FOR FREQUENCY STABILITY VARIANCE MEASURES

2.4.1 Formulation and General Rule ............. 54

2.4.2 Applications $\ldots \ldots \ldots \ldots \ldots \ldots \ldots$

2.5 DESCRIPTION OF THE MEASUREMENT SYSTEM AND THE DEPENDANCE OF THE VARIANCE ESTIMATORS ON $T_{1}, p, r .67$

2.6 SIGNIFICANCE AND APPLICABILITY OF TIME DOMAIN STABILITY MEASURES WITH RESPECT TO FREQUENCY DOMANN MEASURES . . . . . . . . . . . . . 70

Appendix 2.1 - Calculation of Integral Terms of the Form

Appendix 2.2 - Calculation of Summations of Binomial Products ....................... 78

Appendix 2.3 - Relationship to Structure Functions . . . . 84

3 DEVELOPMENT OF THE MCMH SYNTHESIZER AND ITS MAIN CHARACTERISTICS

3.1 INTRODUCTION $\ldots \ldots \ldots \ldots \ldots \ldots$

3.2 REQUIREMENTS OF A HOPPING SYNTHESIZER EMPLOYED IN A SPREAD-SPECTRUM COMMUNICATION SYSTEM $\ldots \ldots 87$

3.3 FREQUENCY SYNTHESIS BY A MICROPROCESSOR CONTROLLED vCO . . . . . . . . . . . . . . . . . . . . 89 
3.4 THE COUNTER AS A LINEAR SYSTEM AND ITS IMPORTANCE AS A CRITICAL ELEMENT IN THE SYSTEM . . . . . . . . . 93

3.5 ADAPTIVE TECHNIQUES USING TIME-DOMAIN MEASURES TO OBTAIN AN ACCEPTABLE ERROR FOR VCO DRIFT CANCELLATION ....................... 97

3.6 COMPARISON OF MCMH WITH THE OTHER SYNTHESIS TECHNIQUES IN THE CONTEXT OF FREQUENCY HOPPING . . . . 104

4 FREQUENCY CORRECTION USING ADAPTIVE FILTERING TECHNIQUES

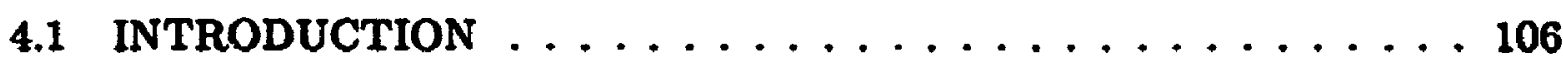

4.2 THE MODIFIED LMS ALGORITHM (MDLMS) . . . . . . . . 107

4.2.1 Mathematical Formulation ............. 107

4.2.2 Convergence Stability . . . . . . . . . . . . 120

4.2.3 Speed of Adaptation . . . . . . . . . . . . 122

4.3 EXPERIMENTAL RESULTS . . . . . . . . . . . 123

Appendix 4.1 - Calculation of Maximum Likelihood Estimates for $\omega_{0}, \omega_{1} \ldots \ldots \ldots \ldots \ldots$

Appendix 4.2 - Reasons for Using $[E\{\hat{R}(n)\}]^{-1}$ Instead of $[\hat{R}(n)]^{-1}$ in Equation (4.5) $\ldots \ldots \ldots \ldots . \ldots 140$ Appendix 4.3 - Derivation of the Elements of $[E\{\hat{R}(k)\}]^{-1} 142$ Appendix 4.4 - Calculation of the Sums $S_{1}(n), S_{2}(n), S_{3}(n) 151$ 


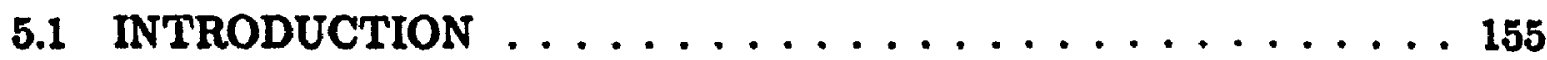

5.2 THE VOLTAGE-CONTROLLED OSCILLATOR . . . . . . . 156

5.3 THE DIGITAL-TO-ANALOG CONVERSION SUBSYSTEM . . . 159

5.4 THE COUNTER $\ldots \ldots \ldots \ldots \ldots \ldots \ldots \ldots$

5.5 THE MICROPROCESSOR AND ITS INTERFACE TO THE REST OF THE SYSTEM . . . . . . . . . . . . 166

5.6 SOFTWARE DEVELOPMENT $\ldots \ldots \ldots \ldots$

5.7 THE OVERALL SYSTEM: TESTING AND MEASUREMENTS . . 175

5.7.1 Hardware Verification . . . . . . . . . . . 175

5.7.2 Selected Hopping Patterns . . . . . . . . . . . . 180

5.7.3 Short-Term Frequency Stability Measurements . . . . . . 181

5.7.4 Real-Time System Adaptation Measurements . . . . . . . 185

Appendix 5.1 - Typical VCO Characteristics Suitable for a $500 \mathrm{MHz}$ Frequency Synthesizer . . . . . . . . . 191 Appendix $5.2 \ldots \ldots \ldots \ldots \ldots \ldots$ Appendix $5.3 \ldots \ldots \ldots \ldots \ldots$ Appendix 5.4 - The I/O Microprocessor Interface . . . . . 197 
6.1 Conclusions . . . . . . . . . . . . . . . . . . 199

6.2 Recommendations . . . . . . . . . . . . . . . 201

REFERENCES . . . . . . . . . . . . . . 203 


\section{List of Figures}

1.1 Phase-noise spectrum of a stable-frequency source. [Manassewitsch 76] 5

1.2 Noise spectrum of a of signal. [Manassewitsch 76] . . . . . . . 5

1.3 An example of a triple-mix synthesis [Manassewitsch 76] . . . . . 9

1.4 Double-mix-divide synthesizer . . . . . . . . . . . . 11

1.5 (a): Digitally controlled PLL synthesizer; (b): Direct digital synthesizer [Phillips 78] . . . . . . . . . . . . . . . . 13

1.6 (a): L-band fine frequency step PLL synthesizer. [Andricos 80]; (b): Fast hopping synthesizer. [Phillips 78] . . . . . . . . . . . 19

1.7 (a): A general model for a spread spectrum communication system; (b): dashed line box for DS systems; (c): Dashed line box for FH

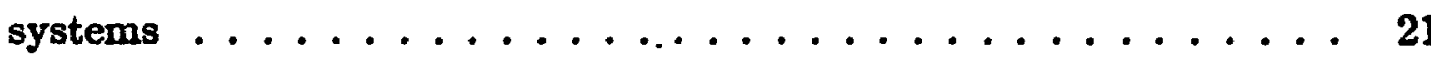

1.8 A hybrid DS/FH transmitter receiver system $\ldots \ldots \ldots \ldots$

1.9 Basic frequency lock synthesizer (courtesy of Dr. R.G. Harrison) . . 27 
2.1 (a): Graphical representation of measurement procedure. [Kalivas 88]; (b): autocorrelation function $R_{\nu}\left(\tau^{\prime}\right)$ compared to the $1-\frac{\left|\tau^{\prime}\right|}{\tau}$ function. [Kalivas 89] .................. 34

2.2 Relationship between IFF $y(t)$ and short term average $\bar{y}_{r}(t) \ldots 36$

2.3 (a): Filter gains $\left|G_{i}\right|^{2}, i=1,2$, obtained with the procedures $P_{1}$ and $P_{2}$; (b): the ideal transfer function representing the optimum $G_{3}(\nu) \quad 45$

2.4 Behaviour of $\hat{\sigma}_{i}^{2}$ as a function of $p \ldots \ldots \ldots \ldots \ldots$

2.5 Interrelation of the various approaches to the study of frequency stability in the time domain (thick arrow represents contribution of this thesis) ......................... 51

2.6 System implementation $\ldots \ldots \ldots \ldots$

2.7 (a): System hopping between two frequencies; (b): typical spectrum of the frequency fluctuations $\ldots \ldots \ldots \ldots \ldots 71$

3.1 Block diagram of the MCMH synthesizer . . . . . . . . . . . . 91

3.2 (a): The counter as a black box; (b): the counter and the "function generator"; (c): the shaded area represents the equivalent ideal filter 94

3.3 General form of adaptive linear prediction filter . . . . . . . . . 102

4.1 Typical measured frequency data (MFD) versus time curve . . . . 111

4.2 Subdivision of MFD into separate regions $\ldots \ldots \ldots \ldots$

4.3 Original and new curves (SMFD) obtained as indicated in Section 4.2.3124 
4.4 (a): MLMS with MFD-straight line and noise; (b): SLMS with MFDstraight line and noise $\ldots \ldots \ldots \ldots \ldots \ldots \ldots$

4.5 (a): MLMS with MFD=straight line and noise; (b): SLMS with MFD=straight line and noise . . . . . . . . . . 129

4.6 (a): "Var505" real MFD; (b): "Var175" real MFD . . . . . . . . . 131

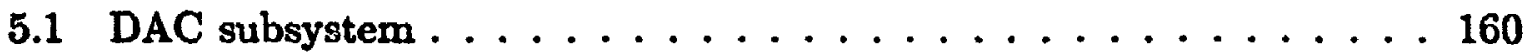

5.2 (a): Step response for linear $6 \mathrm{~dB} /$ octave amplifier; (b): typical settling time characteristics . . . . . . . . . . . . 162

5.3 Schematic of $1 / 0$ interface with system . . . . . . . . 168

5.4 Software generation and interaction with the system $\ldots \ldots \ldots 170$

5.5 Main instructions interfacing FCP to ASCP . . . . . . . 171

5.6 Assembly commanding program (ASCP) structure $\ldots \ldots \ldots 173$

5.7 Counter output waveforms for an IF of $200 \mathrm{MHz} \ldots \ldots \ldots \ldots$

5.8 (a): Algorithm timing (high section of scope trace) (Horizontal: 50 $\mu \mathrm{sec} / \mathrm{div}$, Vertical: $2 \mathrm{~V} / \mathrm{div}$ ); (b): VCO output while it continuously issues one frequency (CTR $=1.0239 \mathrm{GHz}$, Horizontal: $2 \mathrm{MHz} / \mathrm{div}$, Vertical: $\left.10 \mathrm{~dB} / \mathrm{dir}, P_{\text {ref }}=18 \mathrm{dBm}\right) \ldots \ldots \ldots \ldots \ldots \ldots$ 
5.9 (a): VCO hopping from 1017 to $1021 \mathrm{MHz}$; (b): VCO hopping from 1021 to $1017 \mathrm{MHz}$; (c): VCO hopping from 1068 to $1024 \mathrm{MHz}$; (d): VCO hopping from 1024 to $1068 \mathrm{MHz}$. For (a) and (b): Horizontal: $0.2 \mu \mathrm{sec} / \mathrm{div}$, Vertical: $20 \mathrm{mV} / \mathrm{div}$; For (c) and (d): Horizontal: 0.2 $\mu \mathrm{sec} / \mathrm{div}$, Vertical: $50 \mathrm{mV} / \mathrm{div} \ldots \ldots \ldots \ldots$

5.10 (a): MCMH synthesizer hopping between 4 frequencies; (b): Pseudorandom pattern frequency hopping (100 frequencies) . . . . . . 182

5.11 (a): $\hat{\sigma}^{2}$-versus- $p$ for system hopping between two frequencies: $\Delta f=$ $5.5 \mathrm{MHz}$; (b): $\hat{\sigma}^{2}$-versus-p curve for system hopping between two frequencies: $\Delta f=50 \mathrm{MHz} \ldots \ldots \ldots \ldots \ldots$

$5.12 \hat{\sigma}^{2}$-versus-p curves for system hopping between 4 frequencies with $\Delta f=5 \mathrm{MHz}$; (a) Data from $f_{1}=1540 \mathrm{MHz}$ were used; (b) Data from $f_{2}=1550 \mathrm{MHz}$ were used.

5.13 (a): $\hat{\sigma}^{2}$-versus-p curves with and without external disturbance to the V'O' (b) $\hat{\sigma}^{2}$-versus-p for PN frequency hopping (75 frequencies) . . 186

5.14 (a): Initial and final frequency using adaptation (linear offset); (CTR=1.5368 GHz Horizontal $=500 \mathrm{KHz} / \mathrm{div}$ Vertical $=10 \mathrm{~dB} / \mathrm{div}$ ); (b): Initial and final frequency using adaptation (sinusoidal offset) (CTR $=1.5364$ $\mathrm{GHz}$, Horizontal $=200 \mathrm{KHz} /$ div, Vertical $=10 \mathrm{~dB} / \mathrm{div}) \ldots \ldots 187$

5.15 (a): Error between predicted and actual counts from LMS ( $\mu=$ $\left.8 \times 10^{-6}\right)$; (b) Error between predicted and actual counts from LMS $\left(\mu=1 \times 10^{-6}\right)$

5.16 Error when $\mu=1.3 \times 10^{-5}$, creating instability $\ldots \ldots \ldots 190$

5.17 Typical performance of WJ-V801 . . . . . . . . . . . . . . 192 
5.18 Typical performance of VTO-8090 . . . . . . . . . . 193

5.19 The DAC subsystem $\ldots \ldots \ldots \ldots . \ldots \ldots$. . . . . . . . . . . . .

5.20 The high frequency counter (dashed line separates TTL from ECL) . 195

5.21 Parallel terminating scheme . . . . . . . . . . . . . 196

5.22 Detailed diagram of the $\mathrm{I} / \mathrm{O}$ interface $\ldots \ldots \ldots$. . . . . . 198 


\section{List of Tables}

3.1 SYSTEM SPECIFICATIONS . . . . . . . . . . . . . 89 


\section{LIST OF SYMBOLS AND ABBREVIATIONS}

\begin{tabular}{|c|c|}
\hline$A_{0}, A_{1}$ & Expected values of $\hat{\omega}_{0}, \hat{\omega}_{1}$ respectively \\
\hline$A_{00}, A_{11}$ & Expected values of $\hat{\omega}_{0}^{2}, \hat{\omega}_{1}^{2}$ respectively \\
\hline ASCP & Assembly system commanding program \\
\hline ADPLL & All digital PLL \\
\hline$B_{L}$ & Loop bandwidth (Hz) \\
\hline BPF & Band pass filter \\
\hline BF SK & Binary phase-shift keying \\
\hline$B_{\text {ays }}$ & System bandwidth (Hz) \\
\hline BFSK & Binary fr-quency shift keying \\
\hline$B_{H}$ & Hopping bandwidth \\
\hline CTR & Centre of the chart \\
\hline CR & Code rate (symbols/sec) \\
\hline$C_{n}$ & Filter gain coefficients of $G_{3}(f)$ \\
\hline CLB & Closed loop bandwith (Hz) \\
\hline CS & Chip select \\
\hline DDS & Dirtct digital synthesizers \\
\hline DS & Direct sequence \\
\hline DAC & Digital-to-analog converter \\
\hline
\end{tabular}




\begin{tabular}{|c|c|}
\hline$D_{\psi}^{(M)}(\tau)$ & $\begin{array}{l}\text { Mth order structure function of phase instability } \\
\psi(t)\end{array}$ \\
\hline$D_{i j}$ & Minors of $E\{\hat{R}(k)\}$ \\
\hline$D_{0}$ & Determinant of $E\{\hat{R}(k)\}$ \\
\hline$d_{f_{h}}$ & Distance between hopping frequencies \\
\hline$d_{f L O}$ & Distance between the frequencies and the LO \\
\hline ECL & Emitter-coupled logic \\
\hline$E\{\}$ & Expected value operator \\
\hline$f_{\text {in }}$ & Input frequency $(\mathrm{Hz})$ \\
\hline$f_{\text {out }}$ & Output frequency $(\mathrm{Hz})$ \\
\hline$f_{\boldsymbol{r}}$ & $\begin{array}{l}\text { Reference frequency of a PLL; or nominal fre- } \\
\text { quency }(\mathrm{Hz})\end{array}$ \\
\hline FM & Frequency modulation \\
\hline FH & Frequency hopping \\
\hline$f_{v c o}$ & The output frequency of the VCO in a PLL ( $\mathrm{Hz})$ \\
\hline FHO & Frequency hopping oscillators \\
\hline FFT & Fast Fourier transform \\
\hline FLL & Frequency locked loop \\
\hline$F(n)$ & Real function of $\omega_{0}, \omega_{1}, T, \lambda, n$ \\
\hline FCP & Fortran controlling program \\
\hline
\end{tabular}




\begin{tabular}{|c|c|}
\hline$G_{i}(f)$ & Filter gain term \\
\hline$H(f)$ & System transfer function \\
\hline$h(t)$ & Impulse response of a system \\
\hline$h_{j}$ & Oscillator noise coefficients \\
\hline $1 / 0$ & Input-output ports \\
\hline inf & Infimum \\
\hline ITERN & Iteration number \\
\hline IFF & Instantaneous frequency fluctuations \\
\hline IR & Information rate \\
\hline IF & Intermediate frequency \\
\hline$k$ & Number of data points used to calculate $\hat{\omega}_{0}, \hat{\omega}_{1}$ \\
\hline LPF & Low pass filter \\
\hline $\mathbf{k b} / \mathbf{s}$ & Kilobits per second \\
\hline LO & Local oscillator \\
\hline LMS & Least mean square \\
\hline $\mathbf{L}$ & $\begin{array}{l}\text { Number of previous samples used in the adaptive } \\
\text { algorithm }\end{array}$ \\
\hline LSX & The length of stationarity of $X$ \\
\hline$L / D$ & Limiter-Discriminator \\
\hline MLMS & Simplified form of modified LMS algorithm \\
\hline
\end{tabular}


MDLMS

$M S_{0}$

MFD

MCMHS

MS

MSE

$\mathrm{Mb} / \mathrm{s}$

MFSK

NCO

op-amp

PSD

PLL

PD

PCM

PN

PTD

PPI

PC
Modified LMS algorithm

Optimum modulation sensitivity

Measured frequency data

Microprocessor controlled microwave hopping synthesizer

Modulation sensitivity (MHz/V)

Mean square error

Megabits per second

Multiple FSK

Numerically controlled oscillators

Operational amplifier

Power spectral density $(\mathrm{dB} / \mathrm{Hz})$

Phase locked loop

Phase detector

Pulse code modulation

Pseudo-noise

Post tuning drift $(\mathrm{MHz} / \mathrm{sec})$

Programmable peripheral interface

Printed circuit 
$q_{i j}(n)$

QPSK

RF

$R_{y}(\tau)$

ROM

RAM

$R_{\nu}(\tau)$

$\boldsymbol{R}_{\boldsymbol{n}}$

$R_{\phi}(\tau)$

$R_{\Delta v}(k r)$

$\boldsymbol{r}$

RX

$\mathbf{R}$

$\hat{\boldsymbol{R}}(\boldsymbol{k})$

$\hat{r}_{i j}(r)$

$\tilde{\boldsymbol{R}}(\boldsymbol{n})$

$\boldsymbol{R}_{\boldsymbol{f}}$
Total number of frequency measuremer's neecied to characterize the variance

Statistical averages of $\hat{r}_{i j}(n)$

Quadriphase phase-shift keying

Radio frequency

Correlation function of $y(t)$

Read only memory

Random access memory

Autocorrelation of $\nu(t)$

Filter gain coefficients of $G_{3}(f)$

Autocorrelation function of phase noise

Autocorrelation function of $\Delta \psi(t ; \tau)$

Dead time ratio $\left(T_{1} / \tau\right)$

Receiver

Autocorrelation matrix

Estimate of the autocorrelation matrix at kth time instant

Elements of the estimated autocorrelation matrix at the nth time instant

A perturbation matrix

A feedback resistor 
Slew rate $(v / \mathrm{sec})$

SE

Sensitivity

$S_{i}(n), i=1,2,3$

$n$ terms sums as functions of $\lambda$

SER

Sequential regression

STFS

Short-term frequency stability

$S_{\bar{v}_{r}}(f)$

PSD of $\bar{y}_{\tau}(t)(1 / H z)$

SS

Spread spectrum

$S_{v}(f)$

Power spectral density of $y(t)(1 / \mathrm{Hz})$

$S_{\triangleleft}(f)$

Power spectral density of $\phi(f)\left(\mathrm{rad}^{2} / \mathrm{Hz}\right)$

$S_{g}(f)$

Power spectral density of the if signal $(\mathrm{dB} / \mathrm{Hz})$

$T_{\text {acq }}$

Acquisition time for all digital PLL (sec)

$\boldsymbol{T}_{\boldsymbol{p}}$

Pull-in time (sec)

$t_{\text {chip }}$

Frequency chip duration or dwell time (sec)

TD

$T_{1}$

TX

TH

$t_{r}$

VCO

Time domain

Time period between two successive measurements (sec)

Transmitter

Testing hardware

Rise time (sec)

Voltage-controlled oscillator

xxiv 
$V_{t}$

TTL

$W^{*}$

$W(k)$

$\omega_{i}(k)$

$x(t)$

$\hat{x}(k)$

$x(k)$

$X(k)$

$Z_{0}$

$y(t)$

$\bar{y}_{\tau}(t)$

$\bar{y}_{n,} \bar{y}_{\boldsymbol{\tau}}(n)$

$\boldsymbol{Z}_{k, i}$

$x_{i s, i}=1,2,3$

$\boldsymbol{\alpha}_{\boldsymbol{i}}$

$\alpha_{i j}$
Input tuning voltage to the VCO (volts)

Transistor-transistor logic

Optimum weight vector

Weight vector at $\mathbf{k t h}$ time instant

Weight vector component at kth time instant

Continuous signal to the predictor

Predicted value for $x$ at kth time instant

Sampled input signal at kth time instant

Input signal vector at $\mathrm{kth}$ time instant

Characteristic impedance (Ohms)

Instantaneous fractional frequency deviation

Short term average of $y(t)$

Short term average of $y(t)$ at nth interval

Summation factor on variance estimate

Input signal components

Weight factors of the "function generator"

Elements of $[E\{\hat{R}(k)\}]^{-1}$ 


\begin{tabular}{|c|c|}
\hline$\alpha\left(f_{m}\right)$ & $\begin{array}{l}\text { Ratio of power in one noise sideband to the total } \\
\text { signal power }(\mathrm{dB} / \mathrm{Hz})\end{array}$ \\
\hline$\delta(t)$ & Kronecker's delta function \\
\hline$\Delta \omega_{p 0}$ & Pull-out range (rad/sec) \\
\hline$\hat{\nabla}_{k}$ & Estimate of the gradient at kth time instant \\
\hline$\epsilon(k)$ & The error at kth time instant \\
\hline$\Delta f_{r m s}$ & rms frequency deviation $(\mathrm{Hz})$ \\
\hline$\zeta$ & Damping factor associated with PLL \\
\hline$\mu$ & Adaptation constant of the LMS algorithm \\
\hline$\mu_{\text {eq }}$ & Equivalent adaptation constant \\
\hline$\mu^{+}$ & $\begin{array}{l}\text { Adaptation constant of the orthogonalized LMS } \\
\text { algorithm }\end{array}$ \\
\hline$\mu \mathbf{P}$ & Microprocessor \\
\hline$\mu \mathrm{sec}$ & Microsecond \\
\hline$\nu(t)$ & Noise term with zero mean \\
\hline $\boldsymbol{\xi}$ & Mean square error \\
\hline$\sigma_{y}^{2}(\tau, N, T), \sigma_{1}^{2}\left(\tau, p, T_{1}\right)$ & Sample variance \\
\hline$\sigma_{\bar{y}_{r}}^{2}$ & Variance of $\bar{y}_{\tau}(t)$ \\
\hline$\hat{\sigma}_{i}^{2}\left(\tau, p, T_{1}\right)$ & Variance measures $(i=1,2,3)$ \\
\hline$\sigma_{i}\left(\tau, p, T_{1}\right)$ & Estimates of the variance $(i=1,2,3)$ \\
\hline
\end{tabular}


$\hat{\sigma}_{i, j}^{2}\left(\tau, p, T_{1}\right)$

$\boldsymbol{\tau}$

$\phi(t)$

$\phi_{i}$

$\omega_{n}$

$\omega_{0}$

$\omega_{1}$

$\hat{\omega}_{0}$

$\hat{\omega}_{1}$
Generalized variance estimate

Averaging or counting interval (sec)

Phase noise term

Mixing constants

Loop natural frequency $(\mathrm{Yz})$

Constant term of the measured frequency data (Hz)

Slope of the measured frequency data $(\mathrm{Hz} / \mathrm{sec})$

Estimate of $\omega_{0}$

Estimate of $\omega_{1}$

xxvii 


\section{Chapter 1}

\section{STABILITY OF OSCILLATORS AND FREQUENCY SYNTHESIS}

\subsection{INTRODUCTION}

As microprocessors $(\mu P)$ and digital signal processors (DSP) become considerably faster every year, time domain digital processing becomes increasingly advantageous for the design of systems that include feedback loops as their principal elements.

This thesis examines the behaviour of the stability of fast frequency hopping oscillators (FHO) and a resulting new approach to the design of frequency hopping synthesizers using time domain signal processing in a closed frequency locked loop (FLL) configuration. Suitable stability characterization permits the elimination of severe systematic frequency offsets in the new FLL systems when they operate in a closed-loop mode.

In accord with the above general description, this thesis is concerned with three different fields:

1. Measures for frequency stability characterization, 
2. Frequency synthesizers, and

3. Adaptive control.

This chapter briefly considers oscillator frequency stability and measures for the characterization of this quantity. Next, existing methods of frequency synthesis are outlined with emphasis on fast-hopping systems. Potential applications of these systems are briefly presented in the sequel, with emphasis on spread spectrum systems. A new implementation approach, which was first introduced by [Barsalou 82], is presented at the end of this chapter.

The second chapter, perhaps the most important in this thesis, examines frequency stability measures in the time domain and their applicability to FHOs. A particularly useful measure is then p esented in more detail and closed form expressions are derived for it. Its applicability in FHO stability characterization is also discussed.

In Chapter 3, the overall system is presented as a natural application of the theory and measurement process of the previous chapter. The most important elements of this system are discussed. Techniques for frequency correction are also considered.

In Chapter 4, a modified version of a well-known adaptive algorithm is outlined and its resulting faster convergence is demonstrated by simulations.

In Chapter 5 the implementation of the system is discussed in detail and representative measured results are given regarding mainly the time-domain variance characterization. Experimental results concerning the adaptive frequency correction of the system are given as well.

Finally, some conclusions and recommendations for further research are given in the last chapter. 
Before proceeding it should be noted that the appendices appear at the end of the corresponding chapters.

\subsection{FREQUENCY STABILITY OF OSCILLA- TORS}

If the amplitude noise is negligible, output signal of an oscillator is given by:

$$
g(t)=(A+\epsilon(t)) \cos \left(\omega_{0} t+\varphi(t)\right)
$$

where $A$ and $\omega_{0}$ are the nominal amplitude and angular frequency respectively [Rutman 78]. $\epsilon(t)$ is a random quantity denoting amplitude variations around $A$ (amplitude noise). Neglecting amplitude noise for the reasons outlined in [Rutman 78] we define the instantaneous fractional frequency deviation as:

$$
y(t)=\frac{1}{\omega_{0}} \frac{d \varphi(t)}{d t}
$$

The normalization (division by $\omega_{0}$ ) is needed so that the parameter remains unchanged under frequency multiplication and division.

\section{(a) Fourier Frequency Domain}

It has been proposed by the IEEE Subcommittee [Barnes 71] that the Fourier transform $S_{\nu}(f)$ of the correlation function $R_{\nu}(\tau)$ be used as a measure of frequency stability in the frequency domain:

$$
R_{y}(\tau)=<y(t) \cdot y(t-\tau)>
$$




$$
S_{y}(f)=\int_{-\infty}^{\infty} R_{y}(\tau) \exp (-i 2 \pi f \tau) d \tau
$$

$<>$ denotes statistical averages. $S_{y}(f)$ is called the power spectral density (PSD) of $y(t)$. If $S_{\varphi}(f)$ is the spectral density of the phase noise $\varphi(t)$, then from (1.2) and Fourier transform rules we get:

$$
S_{y}(f)=\left(\frac{f}{f_{0}}\right)^{2} S_{\varphi}(f)
$$

where $f_{0}=\frac{\omega_{0}}{2 \pi}$. Following [Rutman 78] we may note that spectral densities are theoretical concepts involving infinite-duration random processes and consenuently true averages. In practice we only have finite-duration phenomena and hence ineasured spectral densities do not accurately represent the actual ones. However the PSD concept provides an important tool for identifying noise processes in actual oscillators and therefore for characterizing their stability. The relationship between the RF spectrum and the phase noise PSD is given by [Rutman 78]:

$$
\begin{aligned}
S_{g}(f) & =\frac{A^{2}}{2} e^{-\left\langle\varphi^{2}\right\rangle}\left\{\delta\left(f-f_{0}\right)+S_{\varphi}\left(f-f_{0}\right)+\right. \\
& \left.+\sum_{n=2}^{\infty} \frac{1}{n !}\left[S_{\varphi}(f) \otimes^{n-1} S_{\varphi}(f)\right]_{f_{0}}\right\}
\end{aligned}
$$

where $\left\langle\varphi^{2}\right\rangle$ is the mean square value of $\varphi(t) .\left[S_{\varphi}(t) \otimes^{n-1} S_{\varphi}(f)\right]_{f_{0}}$ represents $n-1$ convolutions of $S \varphi(f)$ with itself, followed by a translation around the carrier frequency $f_{0}$.

Figures 1.1 and 1.2 show respectively, the phase noise PSD and the RF spectrum of a typical oscillator. In agreement with (1.6) it can be seen that $S_{g}(f)$ shows a decrease (with respect to $S_{\varphi}(f)$ ) of carrier-to-noise power ratio and decrease of 


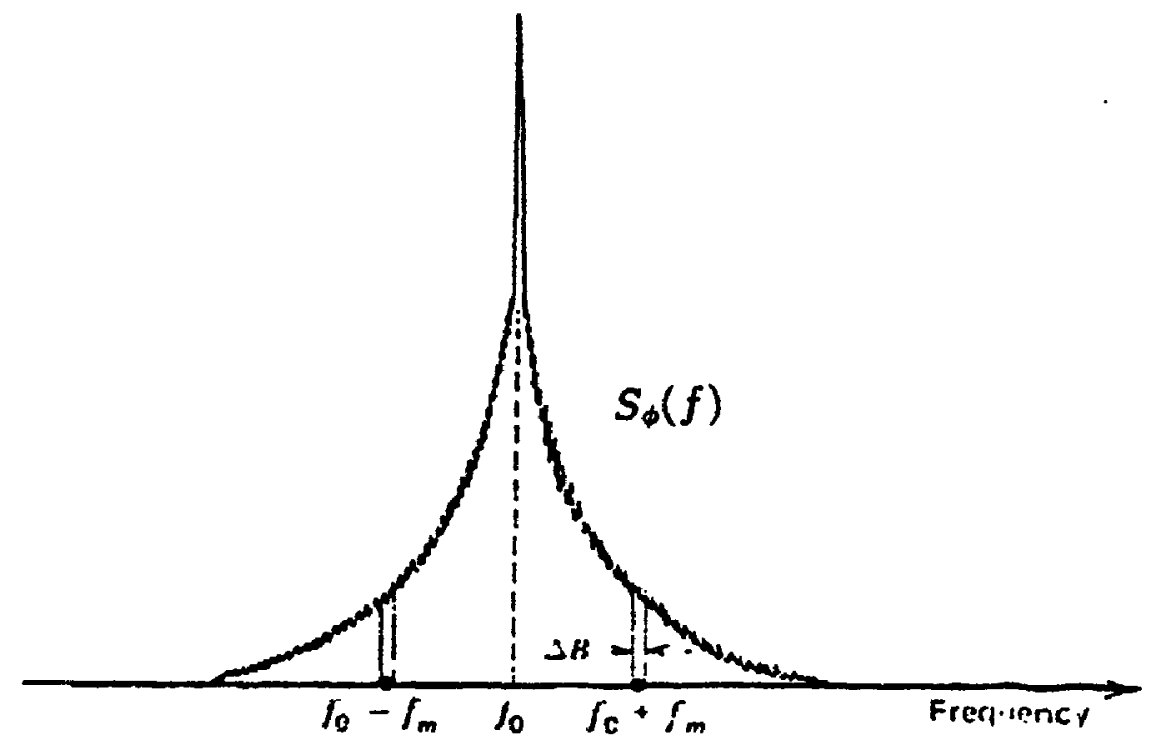

Figure 1.1: Phase-noise spectrum of a stable-frequency source. [Manassewitsch 76]

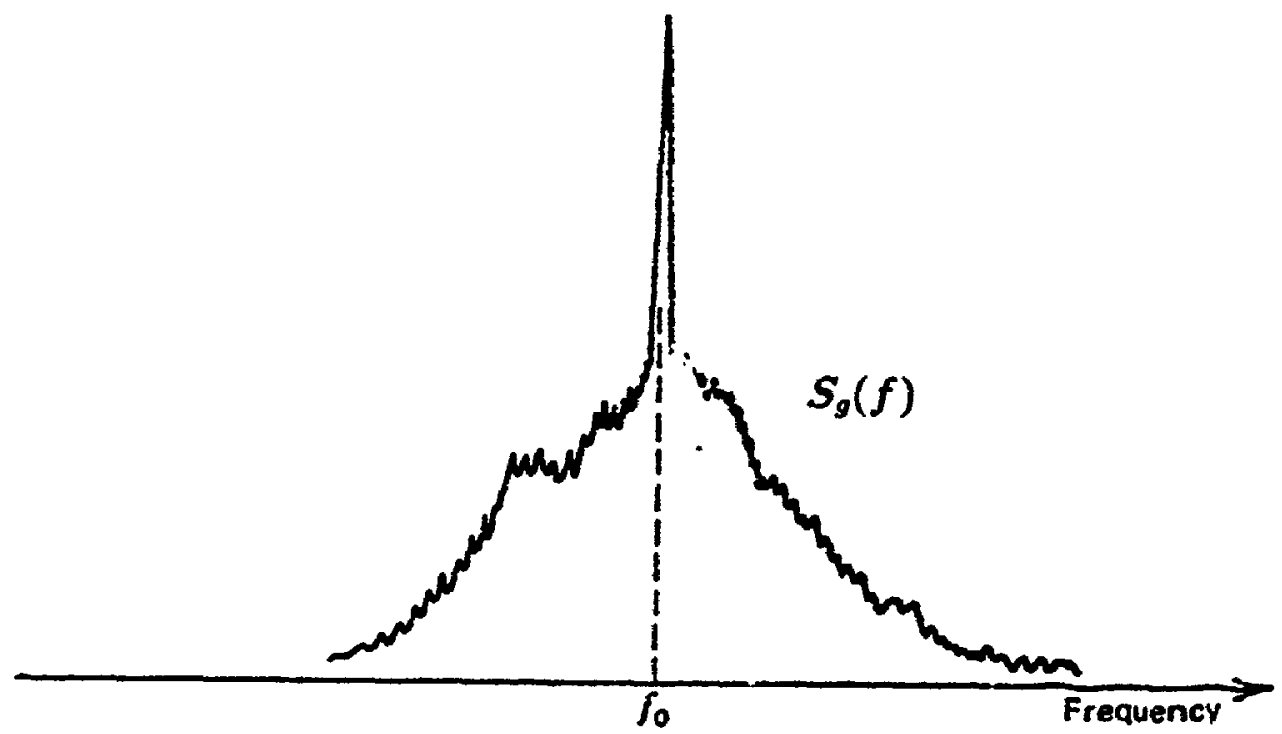

Figure 1.2: Noise spectrum of a rf signal. [Manassewitsch 76] 
the carrier itself due to the $e^{-\left\langle\varphi^{2}\right\rangle}$ term. The noise sideband shape is also changed due to the $n-1$ convolutions.

It is usual to characterize the noise performance of a signal source as the ratio $\alpha\left(f_{m}\right)$ of power in one noise sideband component (located $f_{m}$ away from the carrier) to the total signal power. This implies the assumption that a continuous noise spectrum consists of a very large number of sinusoidal FM sideband components. In this way if $\alpha\left(f_{m}\right)$ is known, one can estimate an equivalent peak rms frequency deviation $\Delta f_{r m s}$ using the following formula:

$$
\alpha\left(f_{m}\right) \simeq 20 \log \left(\frac{\Delta f_{r m s}}{\sqrt{2} f_{m}}\right) d B / H z
$$

\section{(b) Time Domain}

We define $\bar{y}_{k}$, the average fractional frequency offset obtained during the $k$ th measurement interval [Manassewitsch 76, Barnes 71], as:

$$
\bar{y}_{k} \triangleq \frac{1}{\tau} \int_{t_{k}}^{t_{k}+\tau} y(t) d t
$$

where $t_{k+1}=t_{k}+T$, and $T$ is the repetition interval for measurement of the duration $\tau$. The quantity $\bar{y}_{k}$ is related to $M$, the number of zero level crossing points within an interval $\tau$, as measured by a conventional frequency counter:

$$
M=f_{0} \tau\left(1+\bar{y}_{k}\right)
$$

$\tau$ is called the averaging or counting interval. By using the sample variance of the sample of $N$ values: 


$$
\sigma_{y}^{2}(r, N, T)=\frac{1}{N-1} \sum_{n=1}^{N}\left(\bar{y}_{n}-\frac{1}{N} \sum_{k=1}^{N} \bar{y}_{k}\right)^{2}
$$

Allan and Barnes [Allan 66], [Barnes 71] have shown that for limited values of $N, T$ and $\tau$ the limit

$$
<\sigma_{y}^{2}(\tau, N, T)>=\lim _{M \rightarrow \infty} \frac{1}{M} \sum_{i=1}^{M} \sigma_{y_{t}}^{2}(N, T, \tau)
$$

exists in many cases whereas the limit

$$
\lim _{N \rightarrow \infty} \sigma_{y}^{2}(\tau, N, T)
$$

does not exist. Equation (1.11) is considered to be the general form of the Allan variance. As will be proved in Chapter 2 , a relation between $S_{y}(f)$ and the general form of Allan variance is the following:

$$
<\sigma_{y}^{2}(\tau, N, T)>=\frac{N}{N-1} \int_{0}^{\infty} \frac{\sin ^{2}(\pi f \tau)}{(\pi f \tau)^{2}}\left(1-\frac{\sin ^{2}(\pi r f N \tau)}{N^{2} \sin ^{2} \pi r f \tau}\right) S_{y}(f) d f
$$

where $r=\frac{T}{r}$ is called the dead time ratio. This relation has been derived in [Barnes 71] and [Boileau 76] but a slightly different proof is given in Chapter 2 following the steps in [Boileau 76]. The important property of this integral (1.13) is that it converges even for spectral densities exhibiting infinite-power properties. This is mainly the reason why $\left\langle\sigma_{y}^{2}(\tau, N, T)\right\rangle$ is the most useful measure for the characterization of frẹuency stability in the time domain.

Finally, it should be emphasized that there is an argument in the literature [Barnes 71], [Boileau 76] about the stationarity of random processes such as $y(t)$. 
What concerns us here is not the validity of the assumption of stationarity of the statistical model [Kartaschoff 78] but whether the integrals used in the expressions of variance and spectral density functions (such as (1.13)) do in fact converge for limited time intervals of observation and for limited frequency ranges.

\subsection{FREQUENCY SYNTHESIS TECHNIQUES AND HOPPING SYNTHESIZERS}

In the context of frequency hopping synthesis it should be stated here that the most stringent limitation for a synthesizer is its switching speed bearing in mind that a large number of frequencies and a wide bandwidth are also required. When the synthesizer is changing frequencies in a pseudorandom manner, the time needed to switch and settle to the new frequency should be in the range of several $\mu$ secs. In criticial applications for frequency hopping synthesizers we also need a method for characterizing their frequency instability under various frequency hopping patterns. But before we discuss it further, the main available techniques for building frequency synthesizers will be outlined. The three main approaches are the Iterated Direct, the Indirect Phase-Locked and the Direct Digital Frequency synthesis techniques . They will be presented briefly. After that hybrid techniques which have been used during the last 10 years to improve on the two main approaches will be outlined.

\subsubsection{Iterated Direct Synthesis}

The two most important methods are the double- or triple-mix and the double-mixdivide approaches [Manassewitsch 76] which use a structure of standard building blocks repeatedly. The basic module of the double-mix approach is indicated by a dashed line at the bottom of Figure 1.3, which illustrates an example of triple-mix synthesis. A series of frequencies is downconverted by mixing with an IF, passed 


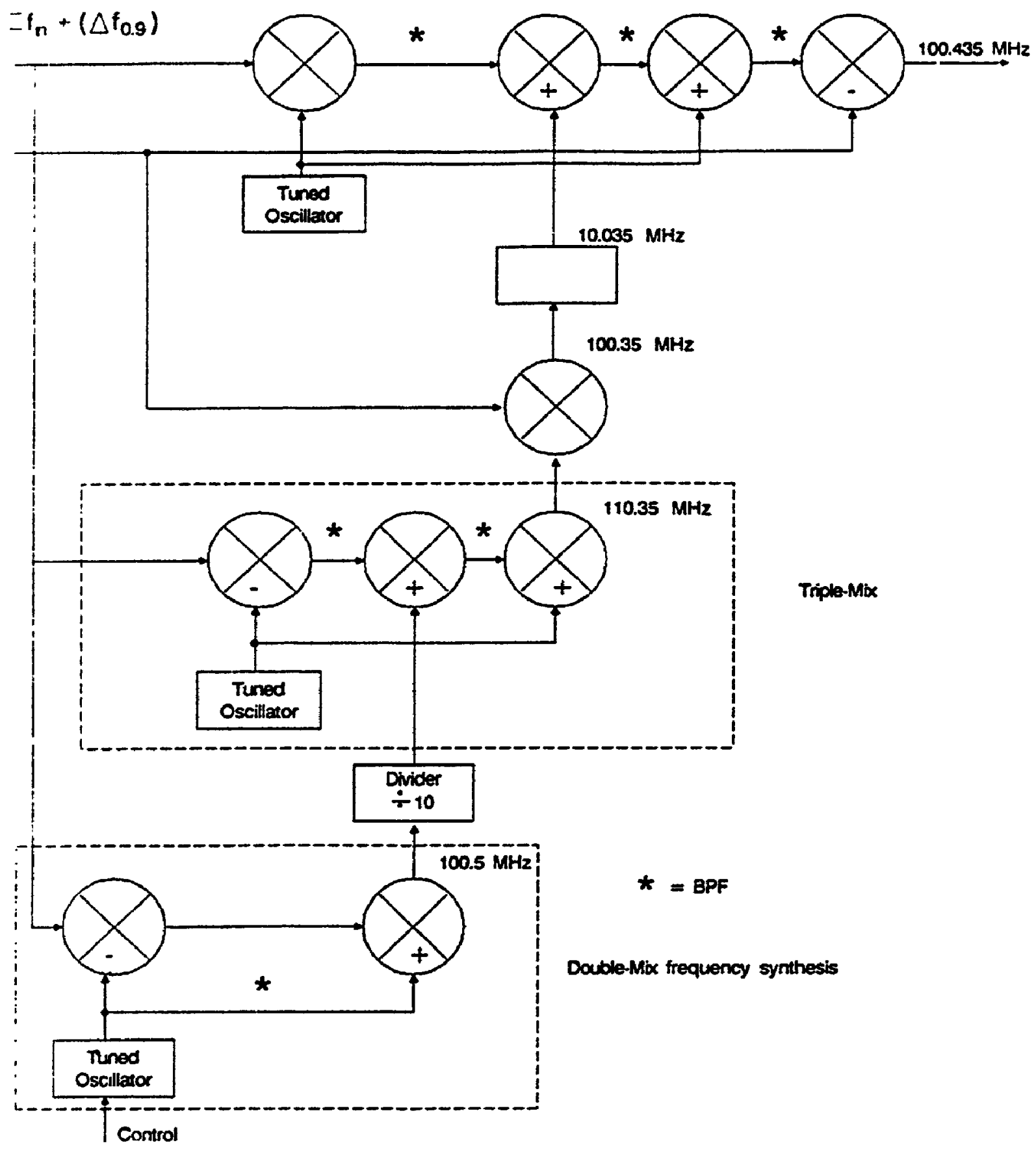

Figure 1.3: An example of a triple-mix synthesis [Manassewitsch 76] 
through a narrow bandpass filter (BPF) which selects one particular frequency and then it is upconverted again by mixing with the same IF to obtain the desired frequency out of the input group of frequencies. In our example this input group is $\sum f_{\text {in }}+\left(\Delta f_{0-9}\right)_{1}$, which means that $f_{\text {in }}+\left(\Delta f_{0}\right)_{1}, f_{\text {in }}+\left(\Delta f_{1}\right)_{1}, \ldots f_{\text {in }}+\left(\Delta f_{9}\right)_{1}$ are all present at the input simultaneously. In the way described above drift cancellation of the IF is achieved. The BPF attenuates all but one input frequency. By down conversion of the input frequencies the effective spacing (which is anticipated as the ratio of the spacing of adjacent input frequencies to the output frequency of the BPF) is increased and therefore the filter is easily realizable. By introducing an additional mixer after the BPF, smaller frequency increments can be achieved. This represents the triple-mixing technique, an example of which is illustrated in Figure 1.3, where 1000 frequencies can be synthesized.

In the double-mix-divide approach, one can see how, by using two sum mixers and a divider, the concept of repeatability can be used to its full potential. Figure 1.4 depicts a synthesizer built using this approach. Dashed lines indicate similar building blocks. There is an input signal $f_{\text {in }}$ and two mixing frequencies $f_{1}, f_{2}$ one of which $\left(f_{2}\right)$ carries 10 frequency increments $f_{2}+\Delta f_{0-9}$. One of these increments is selected at a time instant by a matrix switch. The following equation should be satisfied:

$$
f_{\text {in }}=\frac{f_{\text {in }}+f_{1}+f_{2}}{10}=\frac{f_{1}+f_{2}}{9}
$$

Then the output of the second mixer is divided by 10. The output frequency of each building block is then:

$$
f_{\text {out }}=f_{\text {in }}+\frac{\Delta f_{0-9}}{10}
$$

It should be noted that a divisor other than 10 can also be selected. 


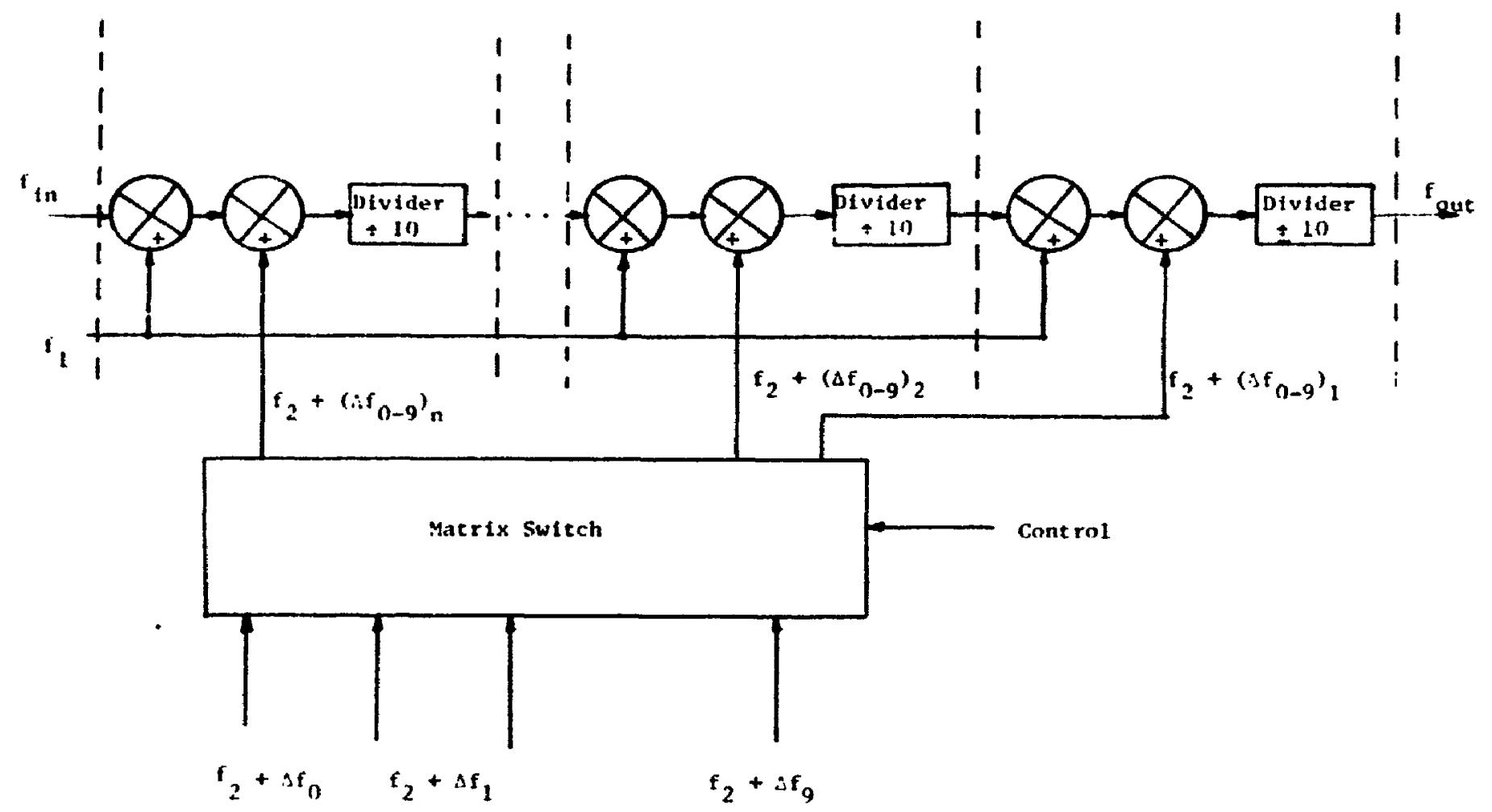

Figure 1.4: Double-mix-divide synthesizer 
The main advantage of the double-mix-divide synthesis over the triple-mix approach is that frequency increments are selected by RF switching and not by the remainder of the various tuned oscillators as is done in the latter method.

\subsubsection{Indirect Phase-Locked Synthesis}

In this type of synthesis a feedback loop is used to lock the output frequency to a reference frequency. To do this, the output frequency of a VCO is downconverted by mixing and dividing. Then it is compared (by multiplication) with an incoming reference signal by means of a phase detector (PD). When the frequency difference of the two inputs of the PD is small, then the PD produces an error voltage in the form of a slowly-varying AC voltage which is low-pass filtered and used to control the VCO by pulling it in lock. A typical block diagram of a phase locked loop (PLL) synthesizer is shown in Figure 1.5(a). When the difference between the frequencies applied to the PD inputs is large, the PD output is a fast-varying AC signal. This signal is attenuated by the LPF, preventing locking.

A PLL is also commonly referred to as a phase-noise filter. It acts as a LPF with respect to noise of the reference signal $f_{r}$ and as a HPF with respect to noise of the VCO (equations (1.10), (1.11) in [Manassewitsch 76]). For locking to occur in the circuit of Figure 1.5(a), the following equation should be satisfied:

$$
N \cdot f_{r}=\frac{f_{V C O}}{N_{0}}-f
$$

By omitting for the moment the divider $\div N_{0}$ and the mixer $\left(N_{0}=1, f=0\right)$ in the feedback path, the above equation indicates that the smallest frequency increment produced by the loop is equal to the reference frequency $f_{r}$ applied to the PD input. For this reason, the VCO frequency $f_{V C O}$ is generally much higher than $f_{r}$. On the other hand, faster acquisition times require higher $f_{r}$. To ensure that the signal will 


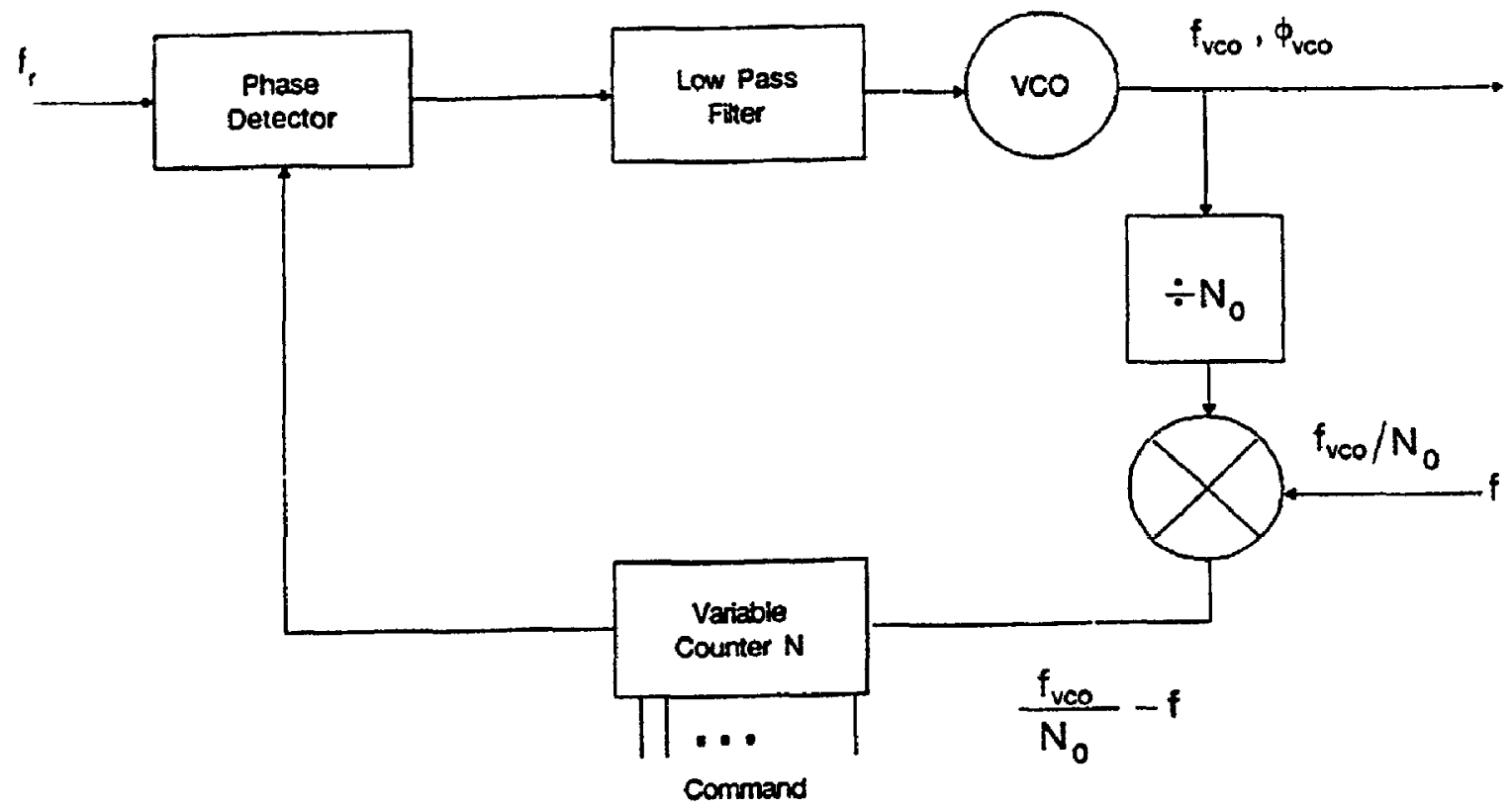

(a)

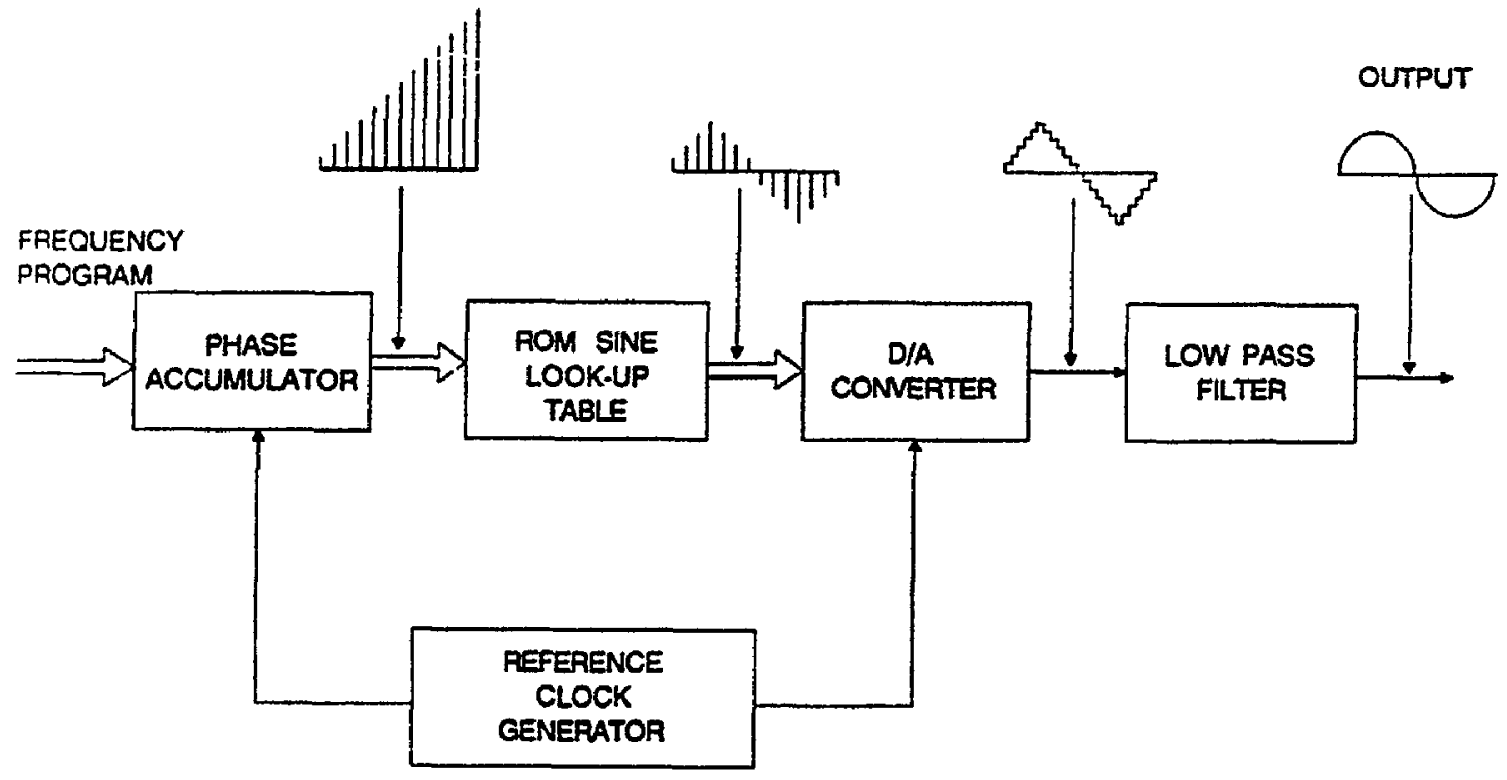

(b)

Figure 1.5: (a): Digitally controlled PLL synthesizer; (b): Direct digital synthesizer [Phillips 78] 
come into the locking range, the locking range can be expanded while at the same time satisfying the above requirements for $f_{r}$, the divider $N_{0}$ and the mixer. The necessary components are included in the circuit of Figure 1.5(a). The difference mixer converts the VCO output frequency down to an acceptable input frequency range for the variable ratio divider $(\div N)$. The $\div N_{0}$ divider is inserted into the loop to increase the operating frequency (locking) range. Notice that now the smaller frequency increment is not $f_{r}$ but $N_{0} \cdot f_{r}$. Therefore it is desirable to restrict $N_{0}$ to small values. If a larger number of frequency increments and a decrease in acquisition time are needed, multiple loops must be used.

Several formulas are given below which interrelate the loop bandwidth, the natural frequency and the pull-in time ([Best 84], [Gardner 79]) for an analog PLL synthesizer.

The maximum practical loop bandwidth is:

$$
B_{L}(\max )=(0.1 \text { to } 0.2) f_{r}
$$

where

$$
B_{L} \simeq \omega_{n}\left(\zeta+\frac{1}{4 \zeta}\right)
$$

in which $\zeta$ is the damping factor. The pull-in time is

$$
T_{P}=\frac{\left(\Delta \omega_{0}\right)^{2}}{2 \zeta \omega_{n}^{3}}
$$

The pull-out range is 


$$
\Delta \omega_{p 0} \simeq 1.8 \omega_{n}(\zeta+1)
$$

Setting $\max \left(\Delta \omega_{0}\right) \simeq \Delta \omega_{p 0}$, eq.(1.19) produces a pull-in time which also represents the major part of the switching time which is equal to:

$$
T_{\text {switch }} \simeq \frac{3}{B_{L}}
$$

These equations can be used to estimate practical limitations concerning the speed and loop bandwidth of a single-loop systhesizer. In indirect one-loop synthesizers there is a trade-off between switching time and frequency resolution (minimum frequency increments). Improved frequency resolution is achieved if $f_{r}$ is reduced since the output frequencies are located $f_{r}$ apart. However because of eq.(1.17), reducing $f_{r}$ will increase the frequency switching time. Even for coarse frequency increments of the order of $200 \mathrm{KHz}\left(f_{r}=200 \mathrm{KHz} B_{L} \simeq 40 \mathrm{KHz}\right)$ the switching time will be approximately $75 \mu \mathrm{sec}$. By switching between two separate PLL synthesizers (ping-ponging) the speed can be considerably increased.

\subsubsection{All-Digital Frequency Synthesizers}

This category consists mainly of the numerically controlled oscillators (NCO) which are referred to as direct digital synthesizers (DDS) in the literature [Phillips 78, Bjerede 76]. Another device which belongs to this category is the all-digital PLL (ADPLL).

A DDS system is shown in Figure 1.5(b). A binary number is incremented by the accumulator with each clock pulse while the size of the increment is dictated by the frequency control input. Frequency resolution depends on the number of bits $N$ in the phase accumulator, since the smallest frequency input is $f_{c} / 2^{N}$. The output 
of the accumulator is used as an input to a sine look-up table contained in a ROM. This digitized sinewave is D/A converted and filtered to get a smooth, noise-free sinewave at the output.

The tuning time of a DDS is mainly determined by the filter. This approach exhibits limitations in terms of bandwidth and output frequencies. The output frequency range can go as high as $100 \mathrm{MHz}$, the main limitation being the $\mathrm{D} / \mathrm{A}$ converter speed. Hotve ser, these devices can be tuned very fast (in 100-200 nsec). Data sheets from recent designs [Stanford Telecommunications 89] demonstrate system settling times of less than 70 nsec operating in the $66-74 \mathrm{MHz}$ range with a resolution of $14 \times 10^{-3} \mathrm{~Hz}$ !

From the above short discussion one can conclude that although DDS are not suitable for microwave frequency synthesis they can be used as the fine-frequencystep portion of a multiloop microwave synthesizer.

The all digital PLL (ADPLL) is a device studied within the last 10 years. It represents the digital counterpart of an analog PLL: the phase detector is replaced by a sampler, the filter becomes digital and the VCO a digitally controlled oscillator [Lindsey 81]. The acquisition time $T_{\text {acq }}$ can be approximated by [Lindsey 78]:

$$
T_{\text {acq }} \simeq N_{\text {acq }} \cdot T
$$

where $N_{\text {acq }}$ is the number of samplings before acquisition. This is at least 10 . The sampling period $T$ is usually taken to be equal to the iv. grse of the bandwidth of the IF filter placed before the sampler.

From the above it can be concluded that the ADPLL apprcach has limitations in terms of maximum output frequency and bandwidth. Usually $100 \mathrm{MHz}$ is an upper limit for the output frequency of these devices [Lindsey 81]. 


\subsubsection{Hybrid Systems}

Although direct microwave synthesizers can be very fast, with switching speeds of the order of a few $\mu$ secs, the existence at the output of many frequencies simultaneously makes it difficult to maintain spurious-free output. The spectral purity depends on many factors such as the oscillator characteristics, RF switch isolation, filter rejection performance and mixer inter-modulation. Phase noise is also considerably increased at the output mainly because of switches' isolation properties and multiplication effects imposed by frequency multipliers. This is especially true for microwave synthesizers.

For all the above reasons the multiple-loop PLL synthesizer approach combined with the DSS approach has become the method of choice curing the last 10 years. By incorporating more than one loop it is passible to relax the stringent limitations due to the trade-off between speed and resolution in PLL systems. It is possible for example to build multiple loop wideband ( $\sim 500 \mathrm{MHz}$ ) microwave synthesizers with $f_{r}$ equal to $1 \mathrm{MHz}\left(B_{L}=200 \mathrm{KHz}\right)$ that will provide settling times of approximately $40 \mu \mathrm{sec}$ [Rhodes 80, Andricos 80]. However the resolution can be increased since the whole system can be driven by a low-frequency direct synthesizer and thus achieve resolution in the range of $1 \mathrm{KHz}$. Alternatively it can be driven by a fine-tuning PLL with resolution of around $500 \mathrm{KHz}$ depending on the minimum resolution required. Figure 1.6(a) is an example of a system synthesizing 350 frequencies with a 517 $\mathrm{KHz}$ resolution and $60 \mu \mathrm{sec}$ settling time. However it should be established here that for any kind of loop the maximum $B_{L}$ is $200 \mathrm{KHz}$ since experience shows that this is the intersection point between the noise spectrum of the VCO and the noise spectrum due to $f_{r}$, the PD and programmable counters. For a synthesizer centered around $1 \mathrm{GHz}$ it is found [Andricos 80 , Rhodes 80] that this point lies at $200 \mathrm{KHz}$ away from the carrier on the phase noise diagram. For smaller offsets, the VCO noise prevails but since the PLL behaves as a HPF with respect to the VCO noise, 


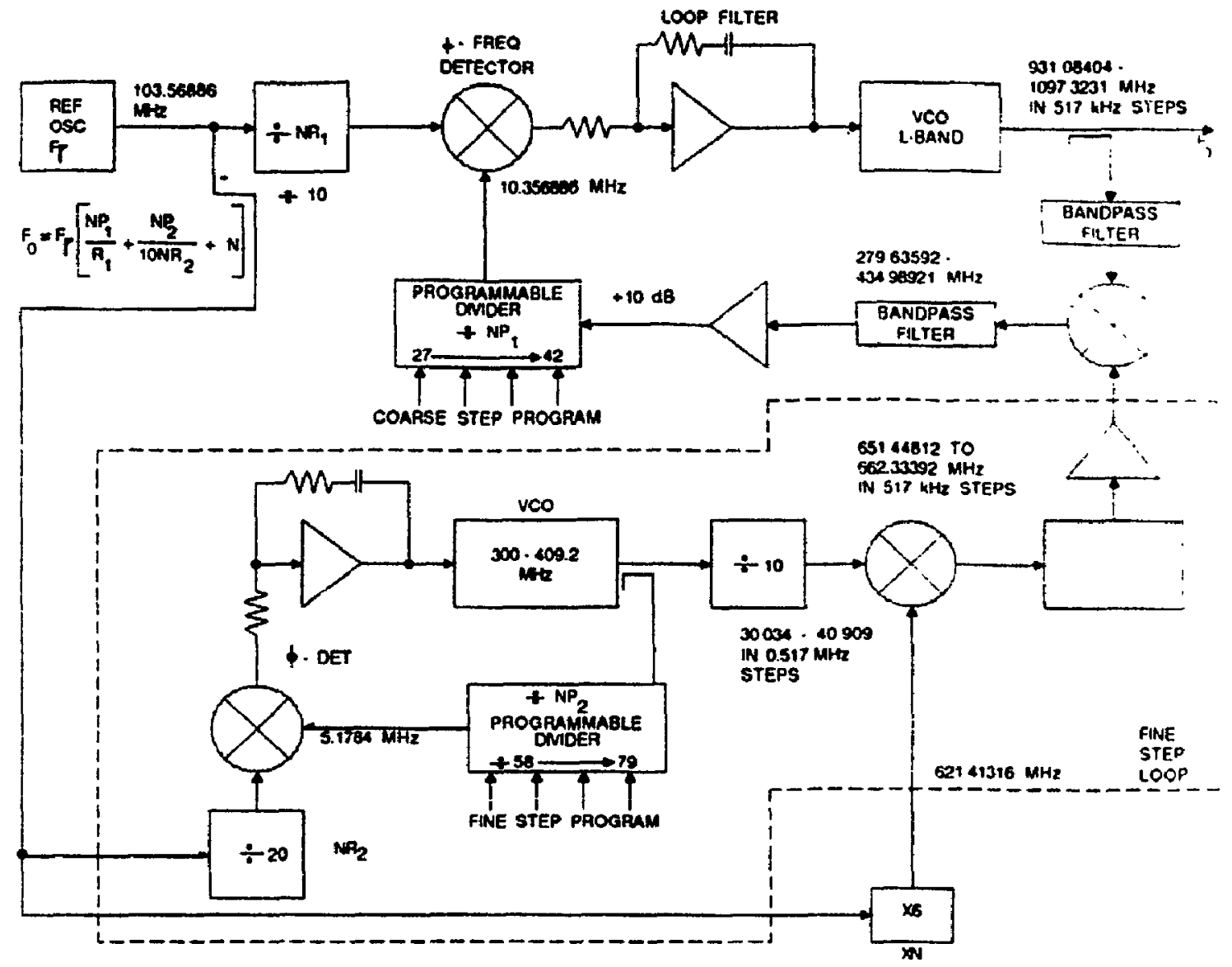

(a)

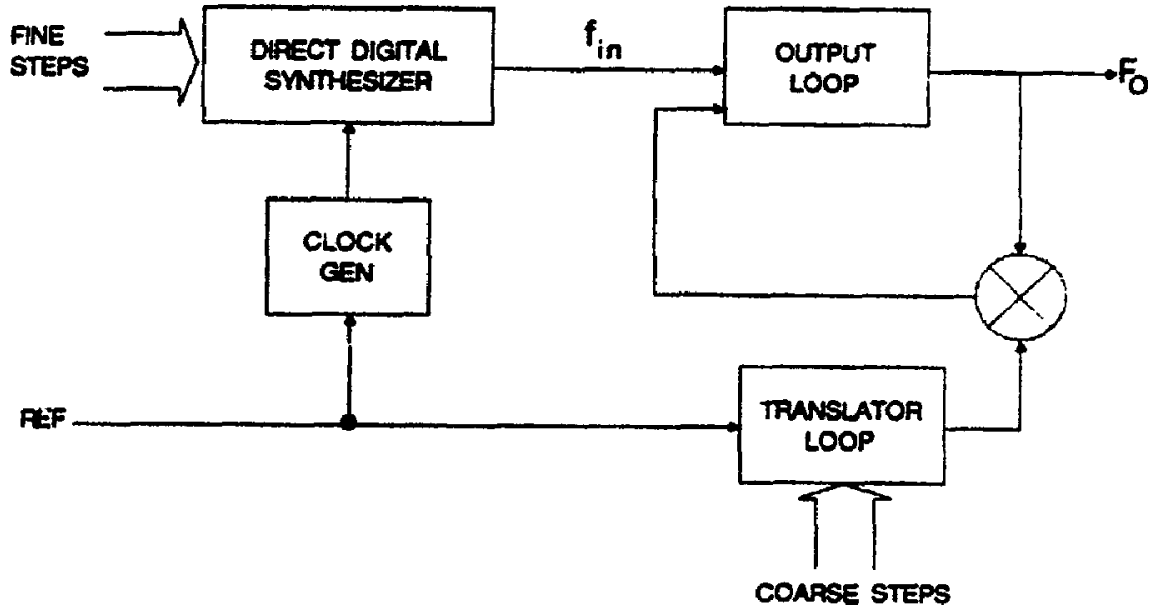

(b)

Figure 1.6: (a): L-band fine frequency step PLL synthesizer. [Andricos 80]; (b): Fast hopping synthesizer. [Phillips 78] 
this noise is eliminated when the PLL is locked. The same thing happens for the PD and counter noise which would prevail above $200 \mathrm{KHz}$ away from carrier but is eliminated due to the operation of the PLL as a LPF with respect to $f_{r}$, PD and programmable counters. Hence we can have a maximum allowaole loop bandwidth $B_{L}$ of $200 \mathrm{KHz}$. Therefore, according to eq.(1.21) the minimum possible $T_{\text {switch }}$ is equal to $15 \mu \mathrm{sec}$ for a frequency step of approximately $1 \mathrm{MHz}$.

Another example of a fast-hopping synthesizer is shown in Figure 1.6(b) where there is a HF band synthesizer ( 2.5 to $29.5 \mathrm{MHz}$ ) in $100 \mathrm{KHz}$ band steps and $10 \mu \mathrm{sec}$ tuning time over any $1 \mathrm{MHz}$ band. Here the system is driven by a low frequency DDS. The frequency resolution is $100 \mathrm{~Hz}$.

\subsection{APPLICATION OF HOPPING SOURCES IN SPREAD SPECTRUM COMMUNICA- TION}

\subsubsection{Definition}

A spread-spectrum (SS) system is a communication system for which the transmitted signal satisfies the following two criteria:

(a) The bandwidth of the transmitted signal (RF bandwidth) must be much greater than the information bandwidth.

(b) The RF bandwidth must be determined by a function which is independent of the information and is known at the receiver.

FM and PCM systems may have greater bandwidths than the information bandwidth. This is the reason why the first criterion only is not sufficient. 


\subsubsection{Basic Spreading Techniques and Applications}

The basic spreading techniques are direct sequence (DS) and frequency hopping (FH). We consider them in turn:

\section{(a) Direct Sequence:}

This uses a code sequence with a much higher bit rate than that of the information signal. Figure 1.7(a) represents a general model of a SS communication system, while Figure 1.7(b) shows the transmitter (dashed line box in Figure 1.7(a)) of a DS-SS system. The modulator is usually of binary phase-shift key (BPSK) or quadriphase phase-shift key (QPSK) type which suppresses the carrier and produces a constant-amplitude envelope. The suppression of the carrier makes it difficult to detect the transmitted signal. The constant envelope ensures that the power is transmitted at its maximum efficiency for the bandwidth used. The RF bandwidth of DS systems is usually [Dixon 84], [Cooper 86] taken as the null-to-null bandwidth of the main lobe in the power spectral density (PSD) function. The PSD has the form of a $(\sin x / x)^{2}$ function. For this reason, in order to have adequate spreading, the PN code rate (CR) must be much higher than the information signal rate (IR). Hence the system bandwidth is

$$
B_{\text {sye }} \simeq 2 \cdot(C R)(H z)
$$

For example in a particular system (discussed later in the chapter) with a $500 \mathrm{MHz}$ bandwidth a code rate of $250 \mathrm{Mb} / \mathrm{s}$ is required. However, this is not acceptable because the clock rates of DS systems are usually for practical purposes limited to $100 \mathrm{Mb} / \mathrm{s}$. Hence, in order to obtain more than $200 \mathrm{MHz}$ bandwidth, a DS system is not adequate.

At the receiver there is a similar PN generator which should be synchronized 


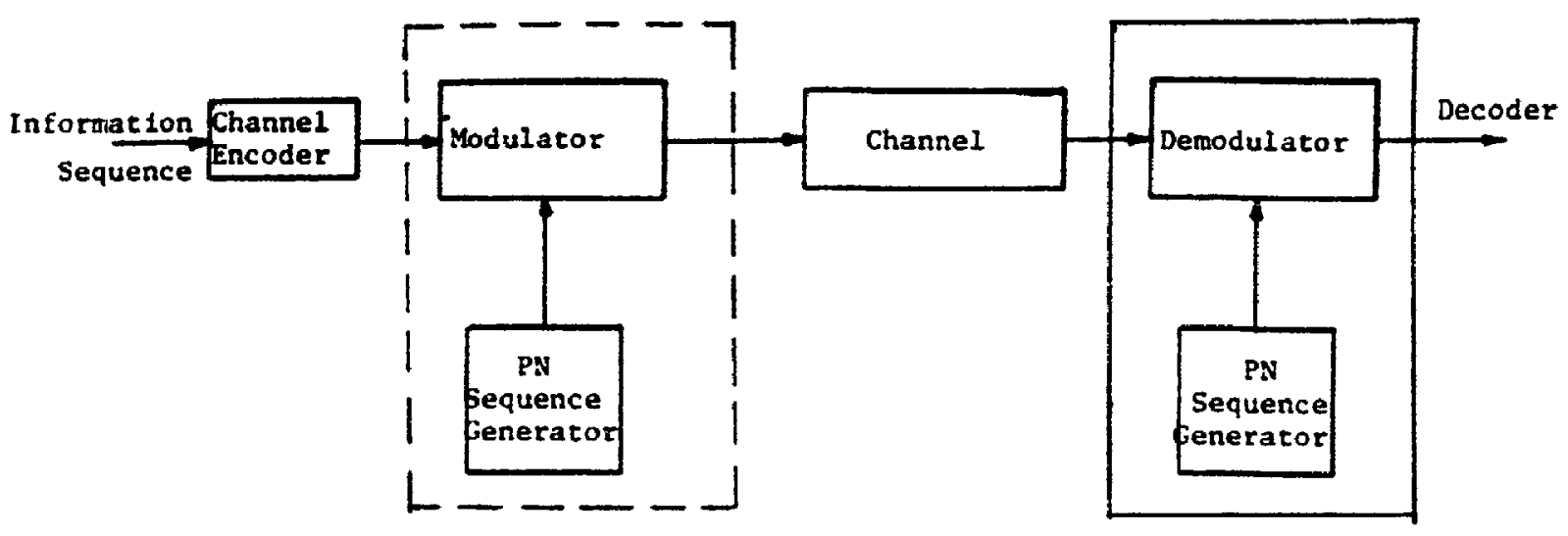

(a)

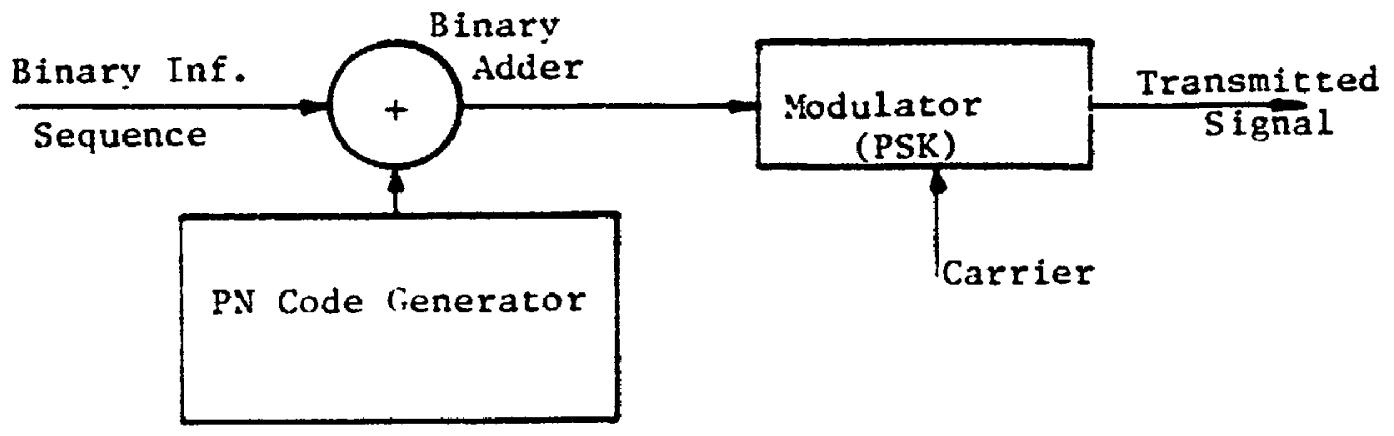

(b)

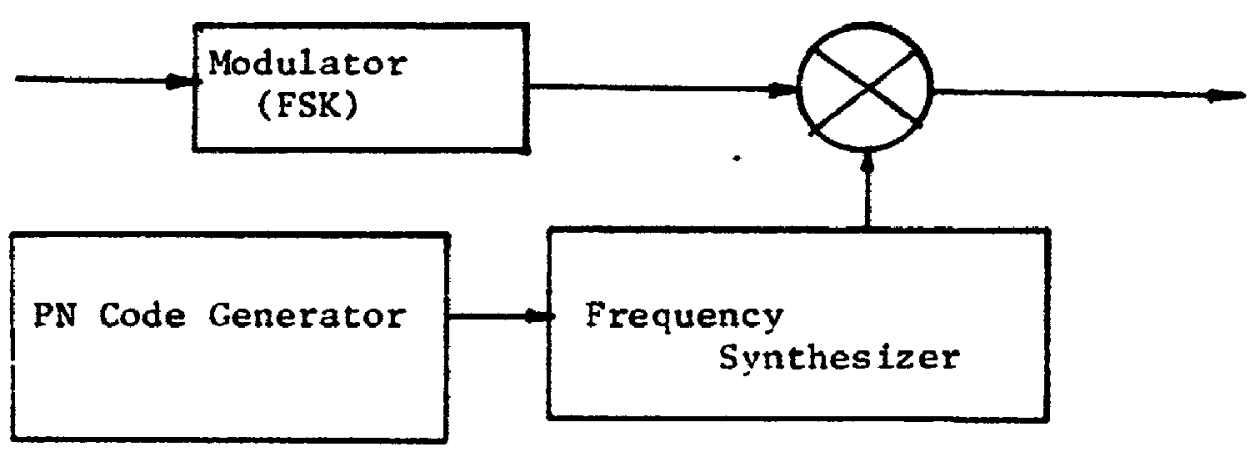

(c)

Figure 1.7: (a): A general model for a spread spectrum communication system; (b): dashed line box for DS systems; (c): Dashed line box for. FH systems 
to the transmitter code in order to reconstruct the useful signal. Hence the receiver transforms the incoming synchronous signals from the coded (RF spread) bandwidth to the baseband. Non-synchronized input signals are spread over the whole PN bandwidth.

\section{(b) Frequency Hrpping (FH):}

The transmitter of a FH system is shown in Figure 1.7(c). In a FH system the available RF bandwidth is subdivided into a large number of contiguous frequency channels (bins). One or more of these frequency bins are sent for each information bit. There are "fast-hop" and "slow-hop" FH systems. In a slow-hop system the hopping rate is lower than or equal to the information symbol rate. In fast-hopping systems the transmitter issues more than one frequency hops per information symbol. The modulation is usually Binary Frequency Shift Key (BFSK) or Multiple FSK (MFSK). If BFSK is used two frequencies are assigned to 1 and 0 message bit states. The output sequences of the PN generator determines the output frequency of the synthesizer which, in turn, is mixed with the output of the modulator and the resultant frequency-translated signal is transmitted over the channel.

If any one frequency bin is interfered with, the information symbols contained in the corresponding time interval will be lost. Hence in order to prevent high bit error rates, error-correction coding of the message should be used, either in the form of an encoder before the modulator or by sending three or more hops per information bit such that if any hop is interfered with, a majority rule could be used in order to get the correct decision about the bit being transmitted. However, in this second case, the system would have to hop three or more times faster. Alternatively, let $N$ be the number of frequencies produced by the synthesizer. The frequencies will be separated by the double-sided hopping bandwidth $B_{H}$ given by the following equation: 


$$
B_{H} \simeq \frac{2}{t_{\text {hop }}}
$$

where $t_{\text {hop }}$ is the hop-frequency duration, which is also known as the dwell time. Then the RF bandwidth is equal to:

$$
B_{\text {oys }}=N \cdot B_{H}
$$

However the frequency bin spacing can be smaller than given by (1.24).

Example: To transmit at an information rate of $10 \mathrm{~kb} / \mathrm{s}$ (message bit duration $\left.t_{m}=\left[10 \times 10^{3}\right]^{-1}\right)$, at three frequency chips per bit, we need a bandwidth $B_{H}=$ $2 / t_{\text {hop }}$. However, $t_{\text {hop }}=\left[3 \times 10 \times 10^{3}\right]^{-1}$. Hence $B_{H}=60 \mathrm{KHz}$. To spread over a $500 \mathrm{MHz}$ bandwidth, 8,333 frequency bins, each $60 \mathrm{KH} / \mathrm{L}$ apart, are needed. The hopping rate is $30 \mathrm{Khops} / \mathrm{sec}$.

\section{(c) Hybrid SS Systems:}

Combinations of two SS systems are often used in order to overcome the shortcomings of a particular technique. The most usual and interesting combination is DS/FH, which is examined in more detail below.

DS/FH systems are used to eliminate the main disadvantages of both DS (fast PN generators) and FH (large number of frequencies) systems, while retaining the same spreading. These disadvantages become more obvious when the required RF bandwidth becomes larger. Hence when the maximum reliable PN generator clock speed is reached in DS or when a limit in the number of frequency hopping channels is obtained, then this kind of hybrid system is especially useful.

Example: For an RF bandwidth $B_{\text {sye }}$ equal to $500 \mathrm{MHz}$ in a DS/FH system, combine a $1 \mathrm{MHz}$ DS system and a 500 channel FH system with 1-MHz channel 
spacing. The DS system will need a $250 \mathrm{MHz}$ clock for the PN generator (very high). The FH system will require 100,000 channels set $5 \mathrm{KHz}$ apart for a $5 \mathrm{KHz}$ information signal.

In Figure 1.8 a hybrid DS/FH modulation-demodulation system is depicted. The DS/FH modulator uses a single PN generator to provide both the direct spreading of information and the frequency hopping pattern. The DS code rate is always much faster than the frequency hopping rate. The main difference from the modulator of a simple DS system is that the carrier frequency is continuously hopping instead of being constant. Hence the frequency difference between successive chips of the synthesizer will be equal to the bandwidth of the DS-spread message (PN code bandwidth) which when mixed with a hopped carrier will produce a bandlimited signal at a particular point in the frequency spectrum at a given time instant. This is indicated by the solid output spectrum in Figure 1.8. The dotted line outputs indicate the various positions of the bandlimited DS-spread message dictated by the frequency hopping pattern.

In Figure 1.8 one can also see the structure of the demodulator where the RF spread signal is mixed with the local reference which is offset by an amount equal to IF frequency. The result of that mixing is then passed through an IF filter whose output is the baseband modulated information.

It should be mentioned here that both the incoming signal and the local reference in the receiver must be spread and hopped. The only differences are the offset IF and the absence of the information modulating the DS sequence.

From Figures 1.7(c) and 1.8 is can be seen that a hopping frequency synthesizer is an integral part of any communication system incorporating frequency hopping. The primary advantages of SS systems are: [Dixon 84], [Cooper 86]

(a) Antijam capability; 


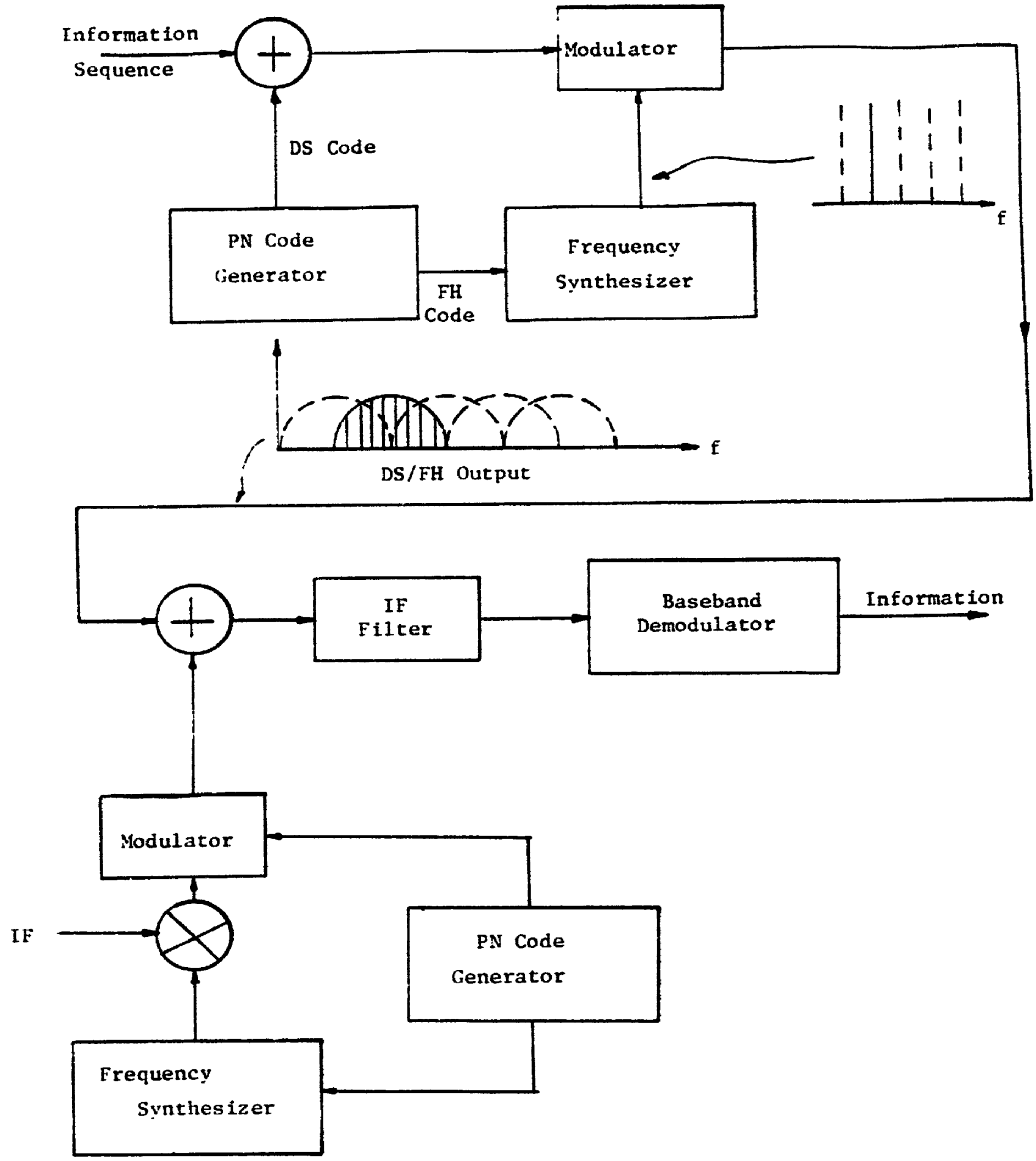

Figure 1.8: A hybrid DS/FH transmitter-receiver system 
(b) Rejection of interference;

(c) Improved spectral efficiency by means of energy density reduction;

(d) Multiple-access capability; and

(e) Resistance to multipath fading.

\subsection{FREQUENCY SYNTHESIS BY A MICROPROCESSOR-CONTROLLED VCO}

The general theory of time-domain frequency stability characterization of hopping sources, can be applied to synthesis of frequency hopping sources as shown in the next chapter. Although it has been introduced by R.G. Harrison and has been described in more detail by [Barsalou 82], it will be briefly outlined here. The main attributes of this synthesizer technique are wide bandwidth ( $\sim 500 \mathrm{MHz})$ and fast switching time ( $1 \mu \mathrm{sec})$.

As shown is Sections 1.3.1 and 1.3.4 the microwave iterative direct frequency synthesis method increases system complexity compared to the PLL approach and reduces spectral purity of the output frequency.

On the other hand, PLL single and multiloop systems (even hybrid) have lower phase noise levels than unlocked sources but they are too slow to be useful at $\mu$ sec hopping rates. (From Section 1.3.4 one can see that the switching time is always in the range of several tens of $\mu$ secs). Our approach involves controlling a wideband microwave VCO through a digital-to-analog converter (DAC). The operation of this system is as follows: (see Figure 1.9)

(a) The tuning voltage-frequency characteristic of the VCO is stored in ROM. 


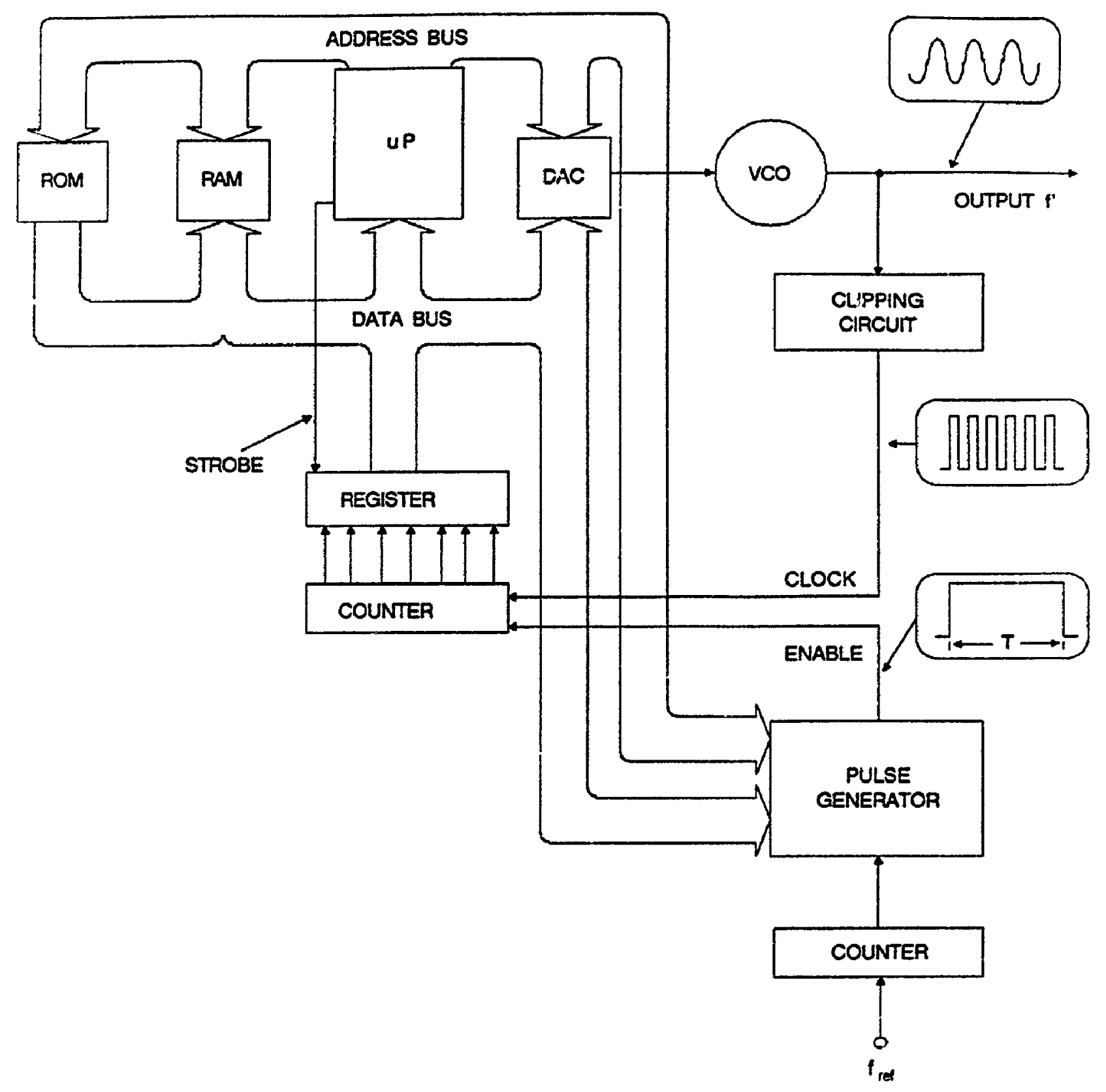

Figure 1.9: Basic frequency lock synthesizer (courtesy of Dr. R.G. Harrison) 
(b) At start-up this is loaded into RAM.

(c) The microprocessor $(\mu \mathrm{P})$ applies an appropriate digital word to the DAC, which causes the corresponding frequency to appear at the output.

(d) The validity of the applied DAC word is verified by counting the output frequency for a short period of time, which in this application, as will be seen later, is close to the dwell time.

(e) The $\mu \mathrm{P}$ calculates the expected count because it "knows" the desired írequency.

(f) The expected count is compared to the actual count and the resulting error is processed to adjust the appropriate digital word in RAM.

The processing of this error in order to adjust the DAC word is the main objective of the $\mu \mathrm{P}$. This adjustment will result in the elimination of frequency fluctuations due to power supply variations and temperature changes. The counter actually represents a feedback element in this system. The output noise and short-term frequency stability which are studied in Chapter 2 are those of the VCO. The short-term accuracy of the synthesizer is a function of the accuracy of the DAC words, which are stored in ROM. The long-term accuracy depends on the updating procedure of these words.

From the above description our system can be related to a PLL system by the fact that they both use feedback. However there are several differences the most important of which are: the nature of the feedback, the way this feedback operates and the ray that the DC tuning voltage is produced and applied to the VCO. Therefore the resulting operating limitations and characteristics of the two systems are different the most important of them being stability, settling time and acquisition time. A last aspect is that our system does not achieve locking in phase but in trequency. 
The approach described here provides less complexity and more flexibility than direct synthesis methods. On the other hand it has a faster settling time than the other indirect and hybrid synthesis methods. Moreover since it does not involve phase locking it eliminates the stability and acquisition time problems associated with the PLL approach. Even the filtering and mixing requirements are reduced compared to the PLL synthesis methods. Its main disadvantage compared to the PLL type of synthesis is that the close-in phase noise will not be filtered out. 


\section{Chapter 2}

TIME DOMAIN

CHARACTERIZATION OF THE SHORT-TERM FREQUENCY STABILITY OF MICROWAVE VCO'S WITH APPLICATION TO HOPPING SOURCES

\subsection{INTRODUCTION}

The main issue addressed in this chapter is the elucidation of a procedure leading to the noise characterization of frequency hopping sources.

In more abstract terms, this chapter presents a detailed study of time domain stability measures necessary for characterizing frequency instability of a class of pulsed signals such as FH signals, radar signals, etc. Ultimately our main interest is pointed at the noise behaviour of frequency agile sources such as pseudorandomly hopping frequency sources. Because of the rapidly increasing data handling capabilities of digital processors and microprocessors it is anticipated that digital methods 
are to be used f : - frequency stability characterization. As an alternative to time domain measures, which are mostly digital processing methods as will be seen further, there is the fast Fourier transform (FFT) frequency domain method. FFT techniques demand a lot more computations and more complex and sophisticated signal processing to perform spectral analysis. However our purpose is not to resolve the PSD of the frequency fluctuations but to obtain a time domain measure of the short term random fluctuations of the oscillator and study how this is affected by increasing the spacing and number of frequencies issued by a hopping oscillator as well as by the dictated frequency pattern. On the other hand equations like (1.13), in their more general form, can be resolved to obtain close approximations for $S_{y}(f)$ if needed.

Until now, various variance measures have been introduced and used [Barnes 71], [Barnes 66], [Allan 66], [Boileau 76], [Boileau 78], [Rutman 78] to characterize the short term frequency stability (STFS) of free running oscillators. In this chapter an attempt is made to apply the existing measures to frequency-hopping sources. Moreover we develop and derive in detail a generalized approach to obtaining closedform expressions for different variance measures. The effectiveness of our approach is demonstrated by applying it to a particular measure. Criteria are also established for choosing the best variance estimator to characterize STFS in the context of FHO.

As pointed out in the lilerature [Buswell 75], [Schoniger 77], [Freq Sources] thermal effects such as changes in junction temperature of the active elements, power dissipation changes and instability of the bias sources are the primary factors contributing to stort-term post tuning drift (PTD). All these changes are the result of commands to change the tuning voltage when the system is to be hopped from one frequency to another. Analog and digital noise coupled to the tuning voltage input of a voltage controlled oscillator can also effectively increase the frequency instability of the system [Manassewitsch 76]. When these effects are taken into account it becomes apparent that the STFS of a hopping system may exhibit behaviour unlike 
that of a free-running frequency source.

In more detail, we first present the definition of the variance, its importance and the role of the frequency counter in its measurement. In the second section, some variance estimators are presented in detail and their correspondence to an equivalent filtering operation is demonstrated. A criterion for making the proper choice is given in the third section. In the fourth section a generalized novel approach to deriving closed-form expressions for frequency stability variance measures is developed. Furthermore, the application of this method to one of the measures demonstrates its validity. After that the dependance of the estimators on certain parameters is estabiished and the description of the measurement system is given. Finally, time-domain and frequency-domain methods are briefly compared to demonstrate the applicability of the first in our application.

\subsection{VARIANCE DEFINITION AND IMPORTANCE, AND THE ROLE OF THE COUNTER IN ITS MEASUREMENT}

The short-term average fractional frequency fluctuation is given, as in eq.(1.8), by

$$
\bar{y}_{\tau}(t)=\frac{1}{\tau} \int_{t-\tau}^{t} y\left(t^{\prime}\right) d t^{\prime}
$$

A counter can measure this quantity by counting the number of zero-level-crossing points of $g(t)$ as defined in (1.1) within the averaging (counting) interval $\tau$. The output frequency is measured every $T_{1}$ seconds for $\tau$ seconds (see Figure 2.1(a)). $T_{1}$ represents the time between two successive measurements of the same output frequency. $p$ represents the number of samples needed to define the estimate of the variance. Considering $y(t)$ as a signal, eq.(2.1) represents a smoothing operation on 

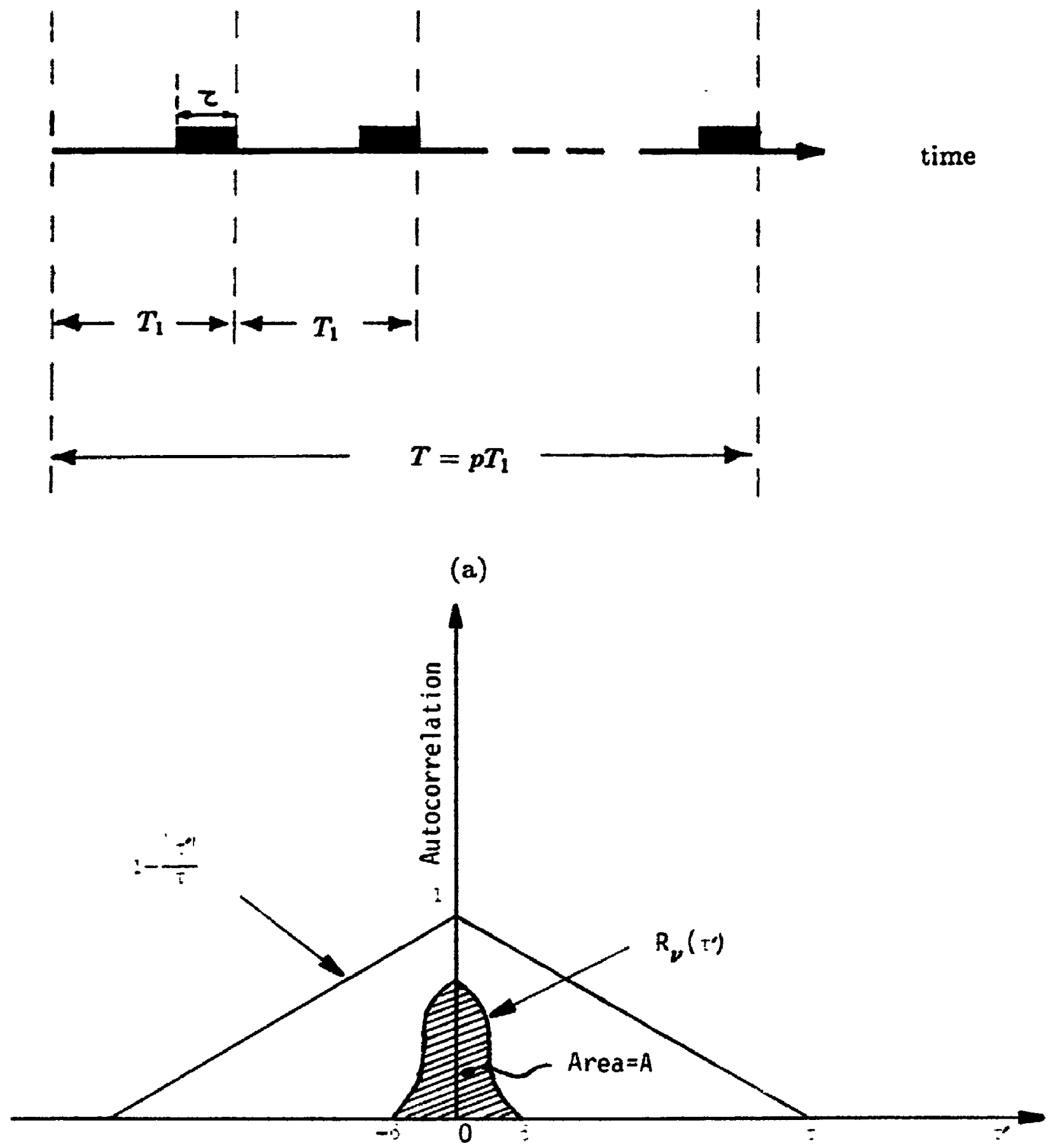

(b)

Figure 2.1: (a): Graphical representation of measurement procedure. [Kalivas 88]; (b): autocorrelation function $R_{\nu}\left(\tau^{\prime}\right)$ compared to the $1-\frac{\left|r^{\prime}\right|}{\tau}$ function. [Kalivas 90] 
$y(t)$. Following [Kalivas 90] we proceed to demonstrate the importance of properly selecting the averaging interval $\tau$.

As an example let us consider that the IFF consists of two terms. The measurable $f(t)$ and the high frequency noise term $\nu(t)$. We want a function $f(t)$ to be measured while we have access to the sum:

$$
y(t)=f(t)+\nu(t)
$$

where $\nu(t)$ represents noise with zero mean and autocorrelation $R_{\nu}\left(\tau^{\prime}\right)$. The shorter $R_{\nu}\left(\tau^{\prime}\right)$ is compared to $\tau$, the closer to white noise is the factor $\nu(t)$. We can assume here that $R_{\nu}\left(\tau^{\prime}\right)$ is of short duration compared to $\tau$ and that its area is equal to $A$ (Figure 2.1(b)). Therefore $f(t)$ contains all the information about $y(t)$. An estimate of $f(t)$ is the short-term average as given by eq.(2.1). The expected value of $\bar{y}_{r}(t)$ is:

$$
E\left\{\bar{y}_{\tau}(t)\right\}=\frac{1}{\tau} \int_{t-\tau}^{t} E\left\{y\left(t^{\prime}\right)\right\} d t^{\prime}=\frac{1}{\tau} \int_{t-\tau}^{t} f\left(t^{\prime}\right) d t^{\prime}
$$

The variance is derived as follows [Kalivas 90]:

$$
\begin{aligned}
\sigma_{\bar{y}_{\tau}}^{2} & =E\left[\bar{y}_{\tau}(t)-E\left(\bar{y}_{\tau}(t)\right)\right]^{2} \\
& =E\left[\frac{1}{\tau} \int_{t-\tau}^{t}\left[f\left(t^{\prime}\right)+\nu\left(t^{\prime}\right)\right] d t-\frac{1}{\tau} \int_{t-\tau}^{t} f\left(t^{\prime}\right) d t^{\prime}\right]^{2}=\frac{1}{\tau^{2}} E\left[\int_{t-\tau}^{t} \nu\left(t^{\prime}\right) d t^{\prime}\right]^{2} \\
& =\frac{1}{\tau^{2}} E\left[\int_{t-\tau}^{t} \int_{t-\tau}^{t} \nu\left(t_{1}\right) \nu\left(t_{2}\right) d t_{1} d t_{2}\right]=\frac{1}{\tau^{2}} \int_{-\tau / 2}^{\tau / 2} \int_{-\tau / 2}^{\tau / 2} R_{\nu}\left(t_{1}-t_{2}\right) d t_{1} d t_{2}
\end{aligned}
$$

The last equation is justified because of the stationarity of the $\nu(t)$ term. By using the same method as in [Papoulis 65] and letting $t_{1}-t_{2}=\tau^{\prime}$ we get: 


$$
\begin{aligned}
\sigma_{\frac{y_{\tau}}{2}}^{2} & =\frac{1}{\tau^{2}} \int_{-\tau}^{\tau}\left(\tau-\left|\tau^{\prime}\right|\right) R_{\nu}\left(\tau^{\prime}\right) d \tau^{\prime} \\
& =\frac{1}{\tau} \int_{-\tau}^{\tau}\left(1-\frac{\left|\tau^{\prime}\right|}{\tau}\right) R_{\nu}\left(\tau^{\prime}\right) d \tau^{\prime}
\end{aligned}
$$

The situation is depicted in Figure 2.1(b) where one can see that $R_{\nu}\left(\tau^{\prime}\right)$ becomes zero for $\left|\tau^{\prime}\right|>\delta$. Hence for $r>>\delta$ we finally get:

$$
\sigma_{\overline{y_{\tau}}}^{2} \approx \frac{1}{\tau} \int_{-\delta}^{\delta} R_{\nu}\left(\tau^{\prime}\right) d \tau^{\prime}=\frac{A}{\tau}
$$

The relation between $y(t), \bar{y}_{\tau}(t)$ and $f(t)$ is shown in Figures 2.2(a) and 2.2(b). From eq.(2.4) one can see that to reduce the variance of $\bar{y}_{\tau}, \tau$ must have a relatively large value. On the other hand $\tau$ should be small enough to ensure that $E\left\{\bar{y}_{r}(t)\right\}$ closely follows $f(t)$ at any instant $t$.

From eq. $(2.1), \bar{y}_{\tau}(t)$ is the output of a filter that has an impulse response as shown in Figure 2.2(c). From Figure 2.2(c) the following equations can be easily extracted:

$$
\begin{gathered}
|H(f)|^{2}=\left(\frac{\sin \pi f \tau}{\pi f \tau}\right)^{2} \\
S_{\bar{y}_{r}}(f)=S_{y}(f)\left(\frac{\sin \pi f \tau}{\pi f \tau}\right)^{2}
\end{gathered}
$$

where

$f$ represents a variable called the Fourier frequency

$S_{y}$ is the PSD of $y(t)$, and 


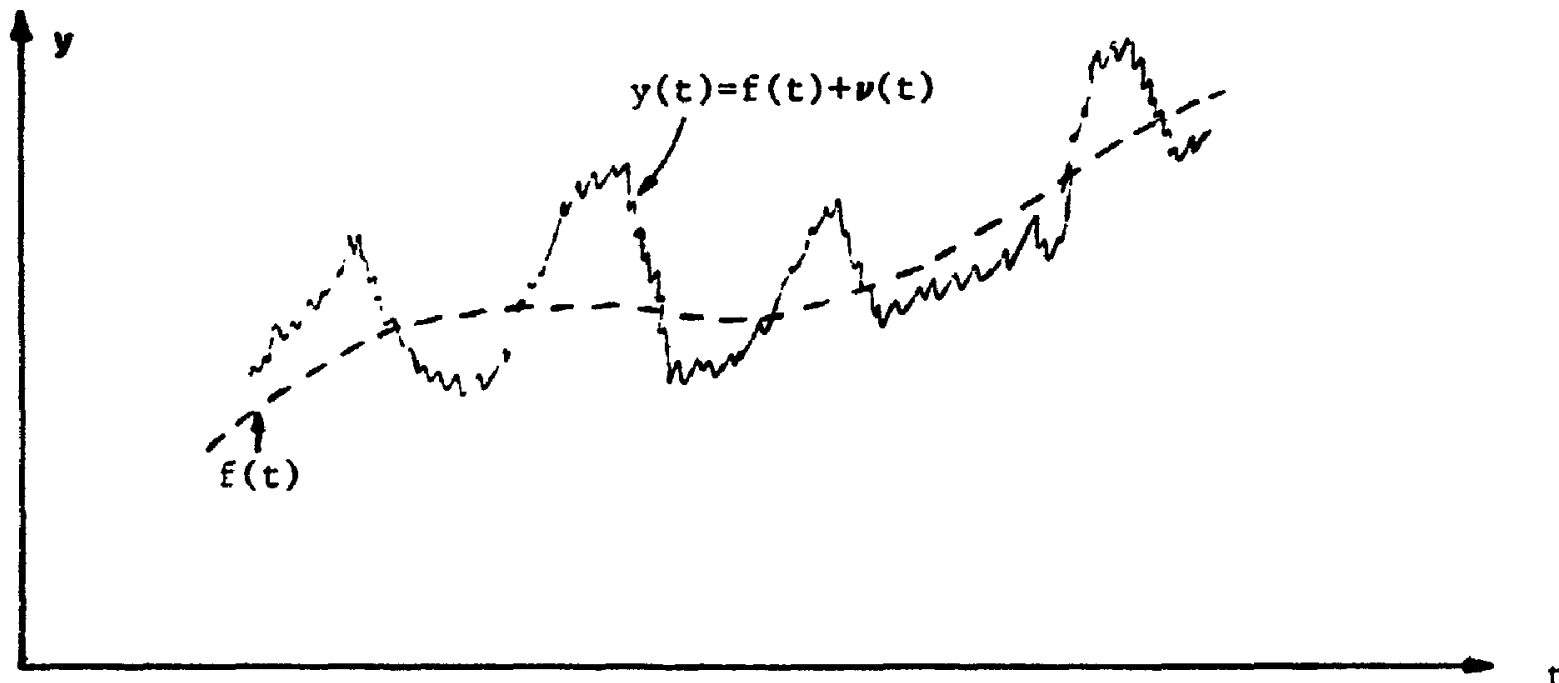

(a)

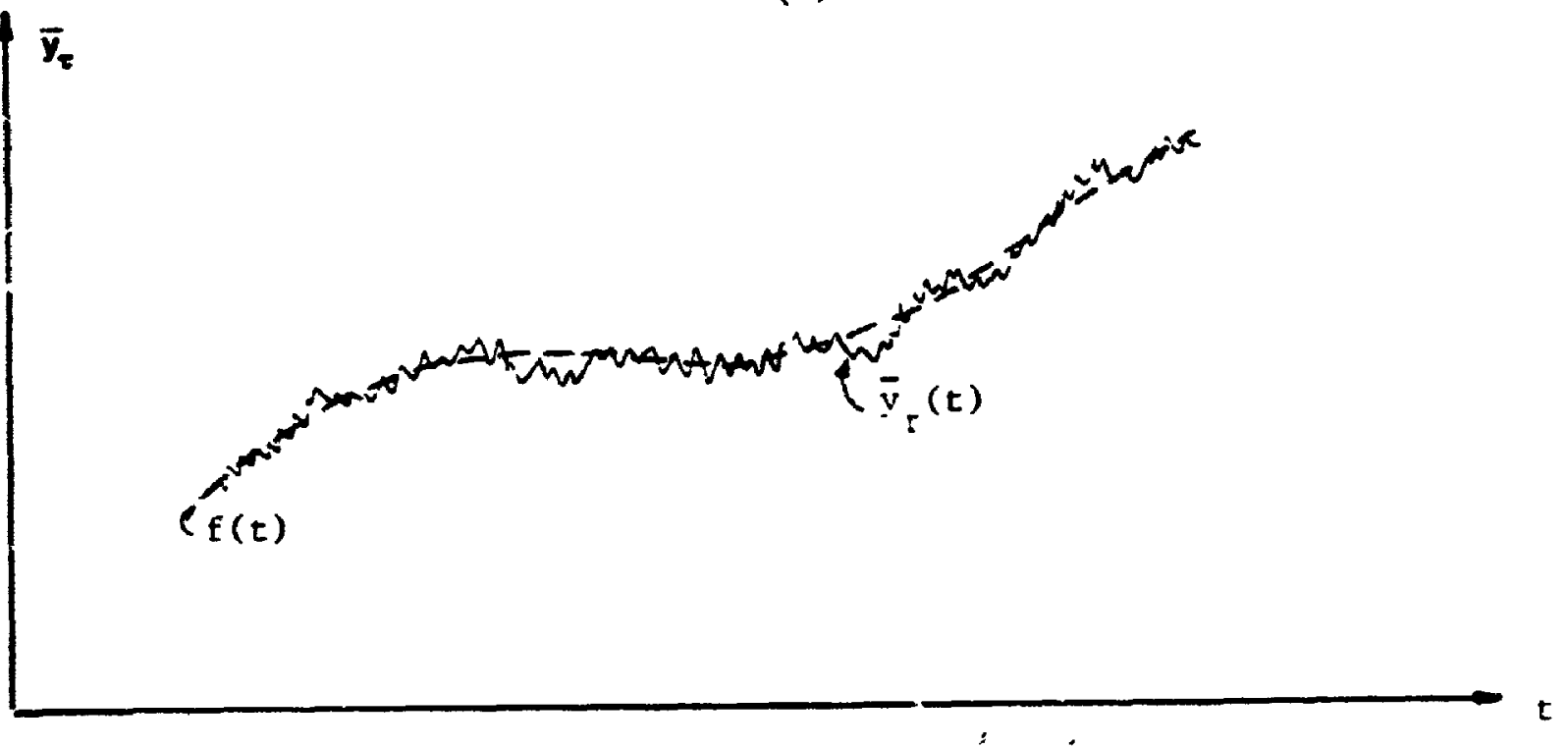

(b) $\quad \therefore$

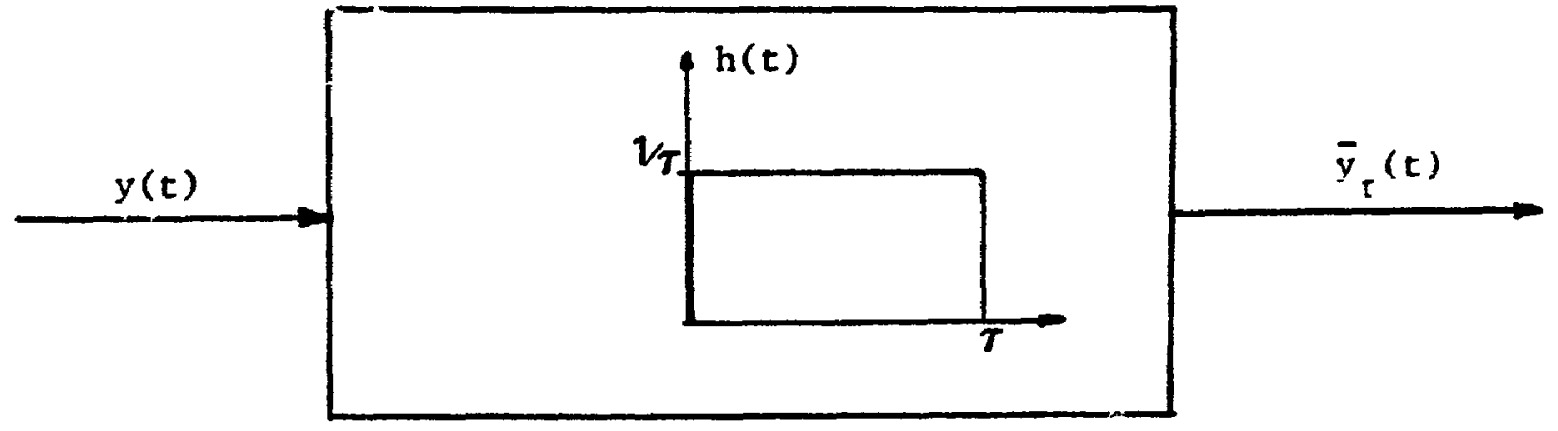

(c)

Figure 2.2: Reiationship between IFF $y(t)$ and short term average $\bar{y}_{r}(t)$ 
$S_{\bar{y}_{\tau}}$ is the PSD of $\bar{y}_{\tau}(t)$.

From eq.(2.6) it can be seen that $\tau$ should be small enough to ensure that shortterm noise, which corresponds to the high-frequency portion of the PSD will not be altered (i.e., filtered out) in any way. One can notice that the observations about how values of $\tau$ affect the spectrum, the variance or the measurement of $y(t)$ itself, are the same above and just after eq.(2.4) because equations (2.4) and (2.6) express actually the same result in the time and frequency domains. Ideally the measures in the two domains (variance and PSD) are related through the following equation:

$$
\sigma_{y}^{2}=\int_{-\infty}^{\infty} S_{y}(f) d f
$$

In reality eq.(2.7) can never be obtained. As a result of the finite number of measurements and of the way measurements are processed to approximate $\sigma_{y}^{2}$, there is an extra term in the integrand which can equivalently be represented as a filter gain term $G_{i}(f)$.

Thus it can be approximated by

$$
\sigma_{y}^{2} \approx \int_{-\infty}^{\infty}\left|G_{i}(f)\right|^{2} S_{y}(f) d f
$$

Concentrating now on the operation of the counter we have:

$$
\begin{aligned}
\bar{y}_{\tau} & =\frac{1}{\tau} \int_{t-\tau}^{t} y\left(t^{\prime}\right) d t^{\prime}=\int_{-\infty}^{\infty} y\left(t^{\prime}\right) h\left(t-t^{\prime}\right) d t^{\prime} \\
& =y(t) \otimes h(t)
\end{aligned}
$$

where 


$$
h(t)= \begin{cases}0 & t<0 \\ 1 & 0<t<\tau \\ \mathbf{T} & t>\tau\end{cases}
$$

From eq.(2.9) we can see that the counter, which gives a multiple of $\bar{y}_{\tau}$, actually performs a convolution of $y(t)$ with a time function $h(t)$ winich can be regarded as the inpulse response of a filter through which $y(t)$ is passed in order to obtain $\bar{y}_{\tau}(t)$. The count measured wy the frequency counter is given by:

$$
M=f_{0} r\left(1+\bar{y}_{\tau}(t)\right)
$$

This is equivalent to passing IFF $(y(t))$ through a filter with impulse response $h(t)$ as given by (2.10).

\subsection{VARIANCE ESTIMATES AND THEIR EQUIVALENT FILTER REPRESENTATION}

Specifically, the principal reason for the existence of the gain term in the variance estimator expressions is the finite number, $p$, of frequency measurements used to characterize the variance measures. However the form of this gain term depends on the way in which these measurements are processed, or to put it in other words on the particular variance measure $\hat{\sigma}_{i}^{2}$ [Barnes 71], [Boileau 76]. Hence the general expression for the variance is given by the following equation:

$$
\hat{\sigma}_{i}^{2}\left(\tau, p, T_{1}\right)=K_{i} \int_{-\infty}^{\infty}\left|G_{i}(f)\right|^{2} S_{\bar{v}_{r(n)}}(f) d f
$$

where 


$$
\bar{y}_{\tau}(n) \Delta \bar{y}_{\tau}\left(t_{n}\right)
$$

In this section, expressions for $\left|G_{i}(f)\right|^{2}$ are established using two different estimates for $\sigma_{y}^{2}$. The detziled derivation of $\left\{\left.G_{1}(f)\right|^{2}\right.$ is also given. A similar method can be used to derive $\left|G_{2}(f)\right|^{2}$. $K_{i}$ is a constant term depending on the variance measure [Kalivas 88].

\subsubsection{Barnes-Allan Variance}

This variance measure was introduced by [Barnes 66] and [Allan 66]. For each set of $p$ samples, the following estimate of the variance is calculated:

$$
\sigma_{1}^{2}\left(\tau, p, T_{1}\right)=P_{1}=\frac{1}{p-1} \sum_{n=1}^{p}\left[\bar{y}_{\tau}(n)-\frac{1}{p} \sum_{k=1}^{p} \bar{y}_{\tau}(k)\right]^{2}
$$

The resulting variance measure is given by:

$$
\hat{\sigma}_{1}^{2}\left(\tau, p, T_{1}\right)=E\left(P_{1}\right)
$$

where $E$ indicates expected value. To derive the gain term $G_{1}(f)$ we proceed as follows: For a random process $x(t)$, the following is true [Papoulis 65]

$$
E\left\{x^{2}(t)\right\}=R_{x}(0)=\int_{-\infty}^{\infty} S_{x}(f) d f
$$

where $n_{x}$ is the autocorrelation and $S_{x}(f)$ the PSD of $x(t)$ respectively.

Eq.(2.14) gives: 


$$
\begin{aligned}
\sigma_{1}^{2}\left(r, p, T_{1}\right) & =\frac{p}{p-1}\left\{\frac{1}{i} \sum_{n=1}^{p}\left[\bar{y}_{\tau}(n)-\frac{1}{p} \sum_{k=1}^{p} \bar{y}_{\tau}(k)\right]^{2}\right\} \\
& =\frac{p}{p-1}\left\{\frac{1}{p} \sum_{k=1}^{p} \bar{y}_{\tau}^{2}(n)-\left(\frac{1}{p} \sum_{n=1}^{p} \bar{y}_{\tau}(n)\right)^{2}\right\} \\
\text { or } \hat{\sigma}_{1}^{2}\left(\tau, p, T_{1}\right) & =\frac{p}{p-1}\left\{E\left[\frac{1}{p} \sum_{k=1}^{p} \bar{y}_{\tau}^{2}(n)\right]-E\left[\left(\frac{1}{p} \sum_{n=1}^{p} \bar{y}_{\tau}(n)\right)^{2}\right]\right\}
\end{aligned}
$$

The first expected value is:

$$
\begin{aligned}
E\left\{\frac{1}{p} \sum_{n=1}^{p} \bar{y}_{\tau}^{2}(n)\right\} & =\frac{1}{p}\left(E\left[\sum_{n=1}^{p} \bar{y}_{\tau}^{2}(n)\right]\right)=\frac{1}{p}\left\{E\left[\bar{y}_{\tau}^{2}(1)\right]+\right. \\
& \left.+E\left[\bar{y}_{\tau}^{2}(2)\right]+\cdots E\left[\bar{y}_{\tau}^{2}(n)\right]+\cdots E\left[\bar{y}_{\tau}^{2}(p)\right]\right\} \\
& =\frac{1}{p}\left\{p E\left[\bar{y}_{\tau}^{2}(n)\right]\right\}=E\left(\bar{y}_{\tau}^{2}(n)\right)
\end{aligned}
$$

Using eqs.(2.16) and (2.18) gives:

$$
E\left\{\frac{1}{p} \sum_{n=1}^{p} \bar{y}_{\tau}^{2}(n)\right\}=E\left[\bar{y}_{\tau}^{2}(n)\right]=\int_{-\infty}^{\infty} S_{\bar{y}_{\tau(n)}}(f) d f
$$

The crlculation of the second expected value in eq.(2.17) is more involved and is as follows. The expression $\sum_{n=1}^{p} \bar{y}_{\tau}(n)$ is produced by estimating the values of $z(t)$ at $t=0$ :

$$
z(t)=\frac{1}{p} \sum_{n=1}^{p} \bar{y}_{\tau}\left(t+n T_{1}\right)
$$


As shown below, $z(t)$ is the output of a filter with input signal equal to $\bar{y}_{\tau}(t)$ and impulse response $h(t)$ given by:

$$
h(t)=\frac{1}{p} \sum_{n=0}^{p-1} \delta\left(t+n T_{1}\right)
$$

This is because:

$$
\begin{aligned}
z(t) & =\bar{y}_{\tau}(t) \otimes h(t)=\int_{-\infty}^{\infty} \bar{y}_{\tau}(t-\alpha) h(\alpha) d \alpha \\
& =\frac{1}{p} \int_{-\infty}^{\infty} \bar{y}_{\tau}(t-\alpha) \cdot \sum \delta\left(\alpha+n T_{1}\right) d \alpha=\frac{1}{p} \sum_{n=0}^{p-1} \bar{y}_{\tau}\left(t+n T_{1}\right) \\
H(f) & =\int_{-\infty}^{\infty} h(t) e^{-j 2 \pi f t} d t=\frac{1}{p} \int_{-\infty}^{\infty} e^{-j w t} \sum_{n=0}^{p-1} \delta\left(t+n T_{1}\right) d t \\
& =\frac{1}{p} \sum_{n=0}^{p-1} e^{-j w\left(-n T_{1}\right)}=\frac{1}{p} \sum_{n=0}^{p-1} \exp \left(j n 2 \pi f T_{1}\right)
\end{aligned}
$$

We will show that:

$$
|H(f)|=\frac{1}{p} \frac{\sin p \pi f T_{1}}{\sin \pi f T_{1}}
$$

Eq.(2.23) represents a geometric series with ratio

$$
r \triangleq e^{j 2 \pi f T_{1}}, \text { where } a=2 \pi f T_{1}
$$

Hence 


$$
\sum_{n=0}^{p-1} r^{n}=\frac{1-r^{p}}{1-r}=\frac{r^{p}-1}{r-1}
$$

Hence

$$
|H(f)|=\frac{1}{p}\left|\sum_{n=0}^{p-1} r^{n}\right|=\frac{1}{p}\left|\frac{\exp \left(j 2 \pi f T_{1} p\right)-1}{\exp \left(j 2 \pi f T_{1}\right)-1}\right|=\frac{1}{p} \frac{\sin \left(\frac{p a}{2}\right)}{\sin \frac{a}{2}}
$$

which is eq.(2.24). From eq. (2.24) the second expected value in eq.(2.17) gives:

$$
\begin{aligned}
E\left[\left(\frac{1}{p} \sum_{n=1}^{p-1} \bar{y}_{r}(n)\right)^{2}\right] & =E\left[z^{2}(0)\right] \\
& =\int_{-\infty}^{\infty} S_{x}(f) d f=\int_{-\infty}^{\infty} S_{\bar{y}_{r}(n)}(f)|H(f)|^{2} d f \\
& =\int_{\infty}^{\infty} S_{\bar{y}_{r}(n)}(f)\left(\frac{1}{p} \frac{\sin p \pi f T_{1}}{\sin \pi f T_{1}}\right)^{2} d f
\end{aligned}
$$

Finally eqs.(2.17) with (2.19) and (2.27) give:

$$
\hat{\sigma}_{1}^{2}\left(\tau, p, T_{1}\right)=\frac{p}{p-1}\left\{\int_{-\infty}^{\infty} S_{\bar{y}_{r}(n)}(f) d f-\int_{-\infty}^{\infty} S_{\bar{y}_{r}(n)}(f)\left(\frac{1}{p} \frac{\sin p \pi f T_{1}}{\sin \pi f T_{1}}\right)^{2} d f\right\}
$$

or

$$
\hat{\sigma}_{1}^{2}\left(\tau, p, T_{1}\right)=\frac{p}{p-1} \int_{-\infty}^{\infty} S_{\bar{y}_{\tau}(n)}(f)\left[1-\left(\frac{\sin p \pi f T_{1}}{p \sin \pi f T_{1}}\right)^{2}\right] d f
$$

or equivalently: 


$$
\left.\begin{array}{l}
\hat{\sigma}_{1}^{2}\left(\tau, p, T_{1}\right)=K_{1} \int_{-\infty}^{\infty} S_{\bar{y}_{\tau}(n)}(f) \cdot\left|G_{1}(f)\right|^{2} d f \\
\quad \text { where }\left|G_{1}(f)\right|^{2}=1-\left(\frac{\sin p \pi f T_{1}}{p \sin \pi f T_{1}}\right)^{2} \\
\quad \text { and } K_{1}=\frac{p}{p-1}
\end{array}\right\}
$$

Equation (2.29) is the same as eq.(10) of [Boileau 78].

\subsubsection{Boileau-Picinbono Variance and Generalization of Variance Estimators}

The Boileau-Picinbono variance estimator has been introduced by Boileau [Boileau 76]. For each set of $p$ samples the estimate of the variance is given by:

$$
\sigma_{2}^{2}\left(\tau, p, T_{1}\right)=P_{2}=\left[\bar{y}_{\tau}\left(\frac{p+1}{2}\right)-\frac{1}{p} \sum_{n=1}^{p} \bar{y}_{\tau}(n)\right]^{2}
$$

where $p$ is an odd integer. The resulting measure is:

$$
\hat{\sigma}_{2}^{2}\left(\tau, p, T_{1}\right)=E\left(P_{2}\right)
$$

Notice that the expression inside the brackets in eq.(2.30) is derived from $\bar{y}_{\tau}(p+1 / 2)$ by means of a linear filter which subtracts from $\bar{y}_{\tau}(p+1 / 2)$ its time average over an observation interval. Using the same procedure as above one can show that [Boileau 76]:

$$
\hat{\sigma}_{2}^{2}\left(r, p, T_{1}\right)=\int_{-\infty}^{\infty} S_{\bar{y}_{r}(n)}(f)\left[1-\frac{\sin p \pi f T_{1}}{p \sin \pi f T_{1}}\right]^{2} d f
$$


or equivalently:

$$
\begin{aligned}
& \hat{\sigma}_{2}^{2}\left(\tau, p, T_{1}\right)=K_{2} \int_{-\infty}^{\infty} S_{\bar{y}_{r}(n)}(f) \cdot\left|G_{2}(f)\right|^{2} d f \\
& \text { where }\left|G_{2}(f)\right|^{2}=\left[\begin{array}{r}
\left.1-\frac{\operatorname{in} p \pi f T_{1}}{p \operatorname{iin} \pi f T_{1}}\right]^{2} \\
\text { and } K_{2}=1
\end{array}\right\}
\end{aligned}
$$

i he filter gain terms $\left|G_{1}(f)\right|^{2}$ and $\left|G_{2}(f)\right|^{2}$ are plotted in Figure 2.3(a) [Boileau 78]. One can sce that they are not good approximations to the function shown in Figure 2.3(b), which is the ideal transfer function needed to obtain an ideal rariance:

$$
\sigma_{y}^{2} \simeq 2 \int_{1 / T}^{1 / 2 T_{1}} S_{\bar{y}_{r}(t)}(f) d f \simeq 2 \int_{1 / T}^{\infty} S_{\bar{u}_{r}(t)}(f) d f
$$

To obtain a close approximation of $\sigma_{y}^{2}$, a more general estimate was introduced by Boileau in 1978 [Boileau 78]:

$$
\left.\begin{array}{l}
P_{3}=\left[\sum_{n=-N}^{N} R_{n} \bar{y}_{\tau}(-n)\right]^{2} \\
\hat{\sigma}_{3}^{2}=E\left(P_{3}\right)
\end{array}\right\}
$$

with

$$
\left.\begin{array}{l}
G_{3}(f)=\sum_{n=-N}^{N} R_{n} \exp \left(j n 2 \pi f T_{1}\right) \\
p=2 N+1
\end{array}\right\}
$$

where $R_{n}$ are given by the following expressions: 


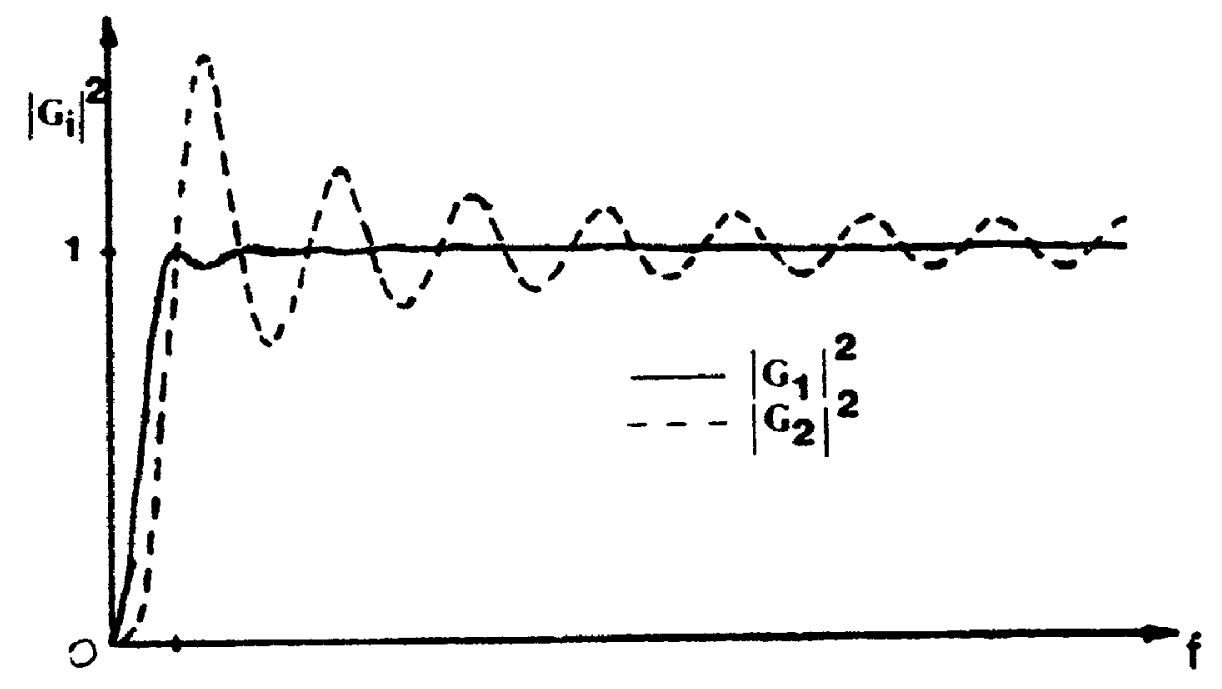

(a)

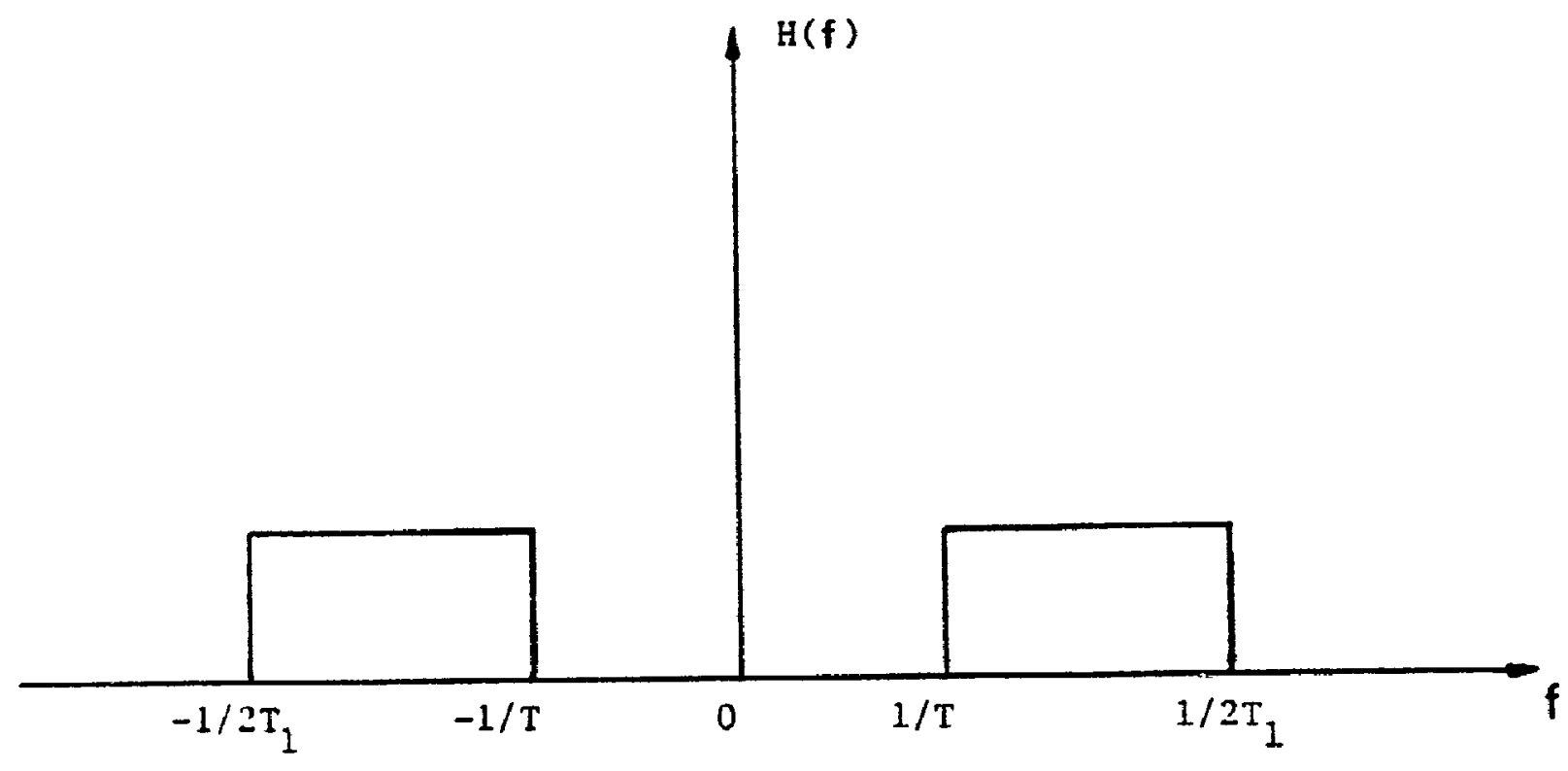

(b)

Figure 2.3: (a): Filter gains $\left|G_{i}\right|^{2}, i=1,2$, obtained with the procedures $P_{1}$ and $P_{2}$; (b): the ideal transfer function representing the optimum $G_{3}(f)$ 


$$
\left.\begin{array}{c}
R_{n}=C_{n}-\frac{1}{2 N+1} \sum_{n=-N}^{N} C_{n} \\
C_{0}=1-2 \frac{T_{1}}{T} \\
C_{n}=-\frac{1}{n \pi} \sin 2 \pi n \frac{T_{1}}{T}
\end{array}\right\}
$$

Similarly the resulting expression for $\hat{\sigma}_{3}^{2}\left(\tau, p, T_{1}\right)$ is:

$$
\hat{\sigma}_{3}^{2}\left(\tau, p, T_{1}\right)=\int_{-\infty}^{\infty} S_{\bar{y}_{\tau}(n)}(f) \cdot\left|G_{3}(f)\right|^{2} d f
$$

One can notice that $P_{3}$ in (2.36) is a generalization of $P_{2}$ and therefore $G_{3}(f)$ in eq.(2.37) is a more general expression of $1-|H(f)|^{2}$ where $H(f)$ is given by eq.(2.23).

The sacrifice for obtaining a variance measure closer to the ideal than $\hat{\sigma}_{1}^{2}$ and $\hat{\sigma}_{2}^{2}$, is the considerable increase in the number of calculations as indicated by eqs.(2.36) - (2.38).

\subsubsection{Criterion for the Choice of a Suitable Variance Estimator}

From the above it is concluded that the different variance measures correspond to different ways of filtering the measurable quantity $\bar{y}_{\tau}\left(t_{n}\right)$. Each of these filters corresponds to a different way of procrssing the measurements in an effort to obtain a suitable variance estimator. We hive just demonstrated three of these measures $(i=1,2,3)$, all of which comply with the generalized equation (2.12).

The expression "suitable variance estimator" has been used several times. Depending on the application, different variance estimators are suitable for different purposes. To be more specific, the usage of eq.(2.12) is twofold [Kalivas 90]:

(a) The first usage is as a measure of the behaviour of $\sigma_{i}^{2}$ as a function of various 
parameters such as $\tau_{1}, p$, and $T_{1}$.

In applications which are directly related to this aspect, the objective is not to obtain $S_{y}(f)$ itself but to test a model for $\hat{\sigma}_{i}^{2}$ with a finite number of measurements. In this case the properties of the filter $G_{i}(f)$ are determined by the definition of the estimate of the variance. This means that no special properties are required of $G_{i}(f)$ because the variance measures here are not intended for spectral analysis. Hence the properties of the filter $G_{i}(f)$ are determined by the definitions of the estimate of the variance. Clearly $G_{1}(f)$ and $G_{2}(f)$ are filters of this type. The main advantage of such filters is the simplicity of the computations needed to obtain $\hat{\sigma}_{i}^{2}\left(\tau, p, T_{1}\right)$. Let us call these, variance measures of the first kind.

(b) The second usage of eq.(2.12) is as a way to acquire a good approximation for the spectral density $S_{y}(f)$ of the frequency fluctuations.

In this case several restrictions are imposed on the filter gain term in order to obtain a specific shape for $\left|G_{i}(f)\right|^{2}$ so as to approximate (2.7) in the best possible way. One such example is the measure $P_{3}$ in eq.(2.36) - (2.38). In this way a globai function corresponding to the ideal variance $\sigma(T \rightarrow \infty)$ can be developed [Boileau 78] despite the fact that this global function will be a "finite time variance" since $T$ will always be finite. However, for this group of variance measures, complicated calculations are needed to obtain $\sigma_{i}^{2}$. Let us call this second group, variance measures of the second kind.

Applications of measures of the first kind are found in the. stability characterization of signal sources. Such characterization usually consists of graphs of $\hat{\sigma}_{i}^{2}$ versus $\tau$, or $p$ or $T_{1}$. This involves measurement of stability of cesium or rubidium frequency staudards [Babitch 74], Radar Sources [Andricos 80], [R. Wiley 82] measurements at very low Fourier frequencies etc. Applications of measures of the 
second kind are found in the spectral analysis region especially close to the carrier where complicated filter expressions are used to increase resolution and to eliminate spurious responses. Such measures are represented by Hadamard Variance and Binominal Weighting [Bough 71], [Rutman 78], [Boileau 78].

In our application concerning frequency-hopping systems we only need an estimate of the short-term noise and not an expression suitable for resolving the spectrum $S_{y}(f)$. Therefore measires of the first kind are adequate for this type of chararterization. Using a suitable value of $\tau$ (the effect of variation of which was demonstrated in Section 2.2) will allow a certain separation between short term fluctuations and slow drifts. This is pictorially shown in diagrams of $\hat{\sigma}_{1}^{2}$ versus $p$ (Figure 2.4).

From eq.(2.6) one can realize that low frequencies are not altered by the shortterm averaging performed by eq.(2.1) and the problem of slow drifts will remain the same for both $y(t)$ and $\bar{y}_{\tau}(t)$. On the other hand the dependance upon $r$ will disappear only if the value of $r$ is small enough so that fast fluctuations including short term noise will not be filtered out. Hence if $r$ is quite large there will not be any separation. This is shown in Figure 2.4 for several values of $\tau$.

A criterion for choosing between all the measures presented above can now be stated [Kalivas 87]:

"For frequency hopping oscillators use a $\hat{\sigma}^{2}$ of the first kind. This measure should exhibit an approximately "horizontal" portion on the $\hat{\sigma}^{2}$ versus- $p$ curve. If such a horizontal region exists, it can be interpreted as the short term variance of the hopping system. If there is not such a region, the value of $\tau$ should be decreased gradually until a horizontal part is obtained on the $\hat{\sigma}^{2}$-versus-p curve." 


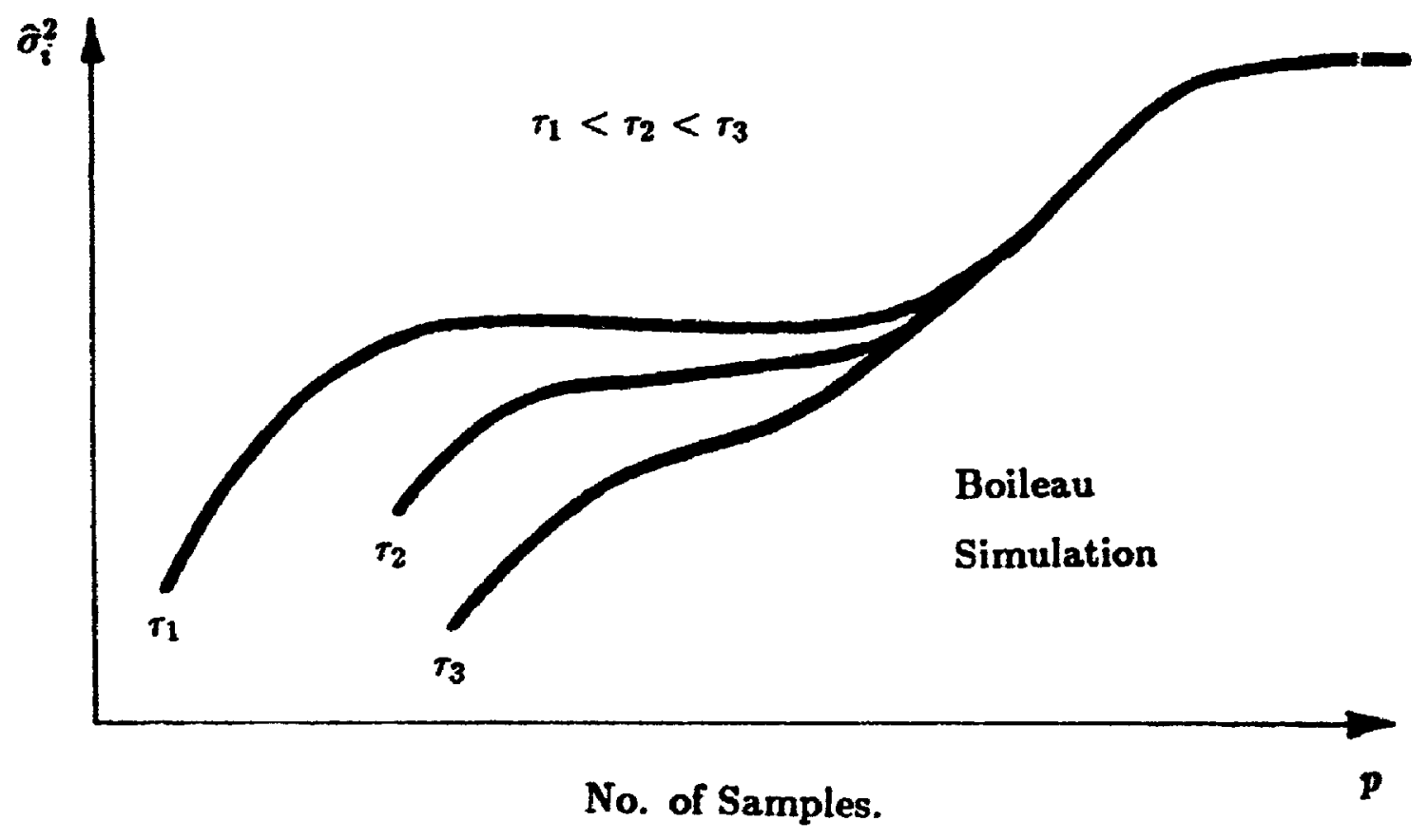

Figure 2.4: Behaviour of $\partial_{i}^{2}$ as a function of $p$ 


\subsection{A NOVEL APPROACH TO DERIVING CLOSED-FORM EXPRESSIONS FOR FREQUENCY STABILITY VARIANCE MEASURES}

The major part of this section is an extension of the work published in [Kalivas 88]. Up to here we have pointed out the significance of the different variance measures and the ability to express all of them in the form of the unifying equation (2.12). Now we concentrate on the possibility of deriving closed-form expressions for the integral (2.12) as functions of $\tau, p$ and $T_{1}$. This is of great importance since it allows the investigation of the behaviour of various $\hat{\sigma}_{i}^{2}$ for different values of $\tau, p$ and $T_{1}$. The more general the method is, the wider applicability it may find for research in TD frequency stability characterization.

Before elaborating on this aspect, let us first look at the directions followed by various researchers on the theory of frequency stability during the last 10 years. This is pictorially demonstrated in Figure 2.5 [Kalivas 88].

The "Sample Variance Approach" [Barnes 71], [Boileau 76], [Barnes 66], [Allan 66] begins from sample variances, proceeds to derivationf of integral expressions of the estimate of the variance (such as (2.12) for various $i$ ), and finally obtains closed form expressions for these estimates [Barnes 71, App.II].

According to the "Transfer Function Approach" [Rutman 74], [Rutman 78], [Boileau 78], the filter term $G_{i}(f)$ is chosen a priori such that certain characteristics are imposed on the estimator. From that point, one can either study the behaviour of $G_{i}(f)$ as a function of $f$ [Rutman 74], [Rutman 78] or proceed one step further, and by using the mathematical technique presented in this section, obtain closed form expressions for the estimate of the variance. Hence, this approach has as its central point the behaviour of the integral (2.12). 


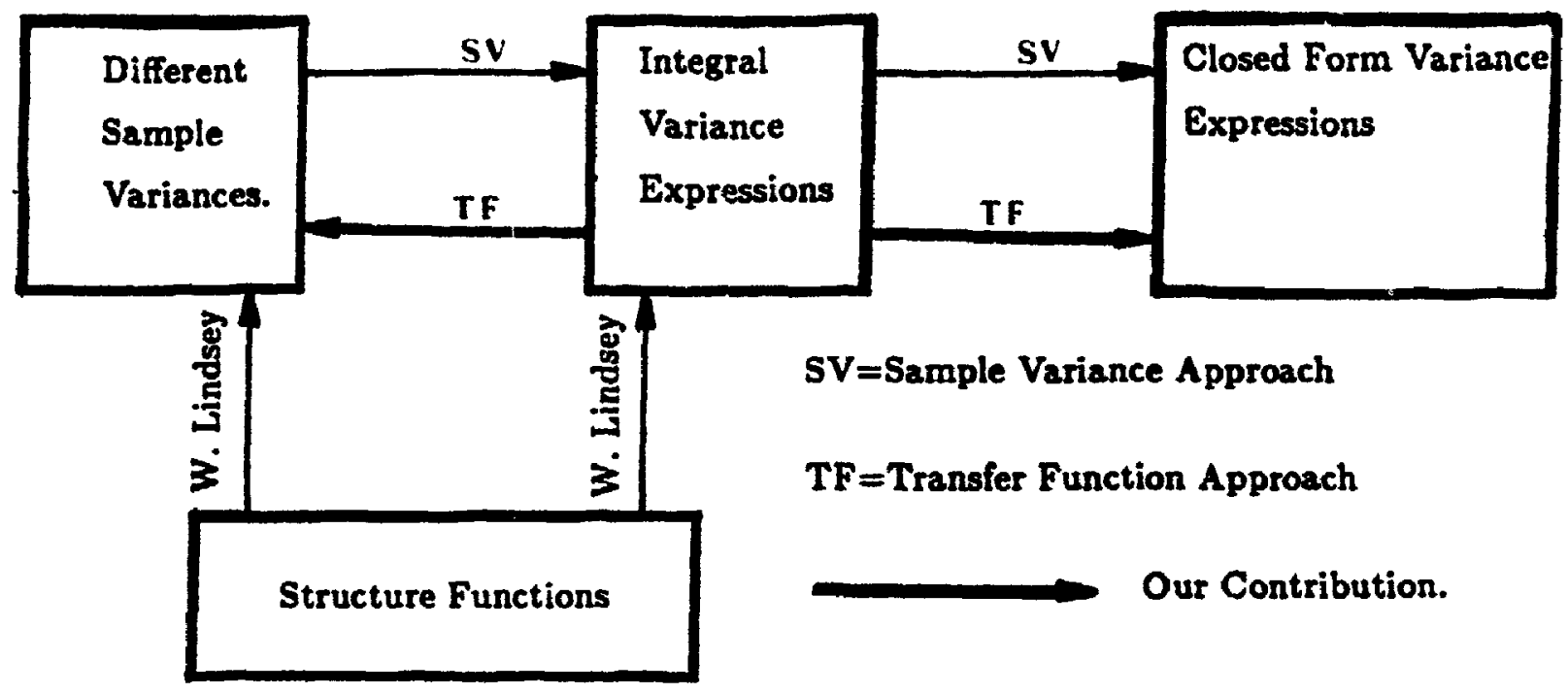

Figure 2.5: Interrelation of the various approaches to the study of frequency stability in the time domain (thick arrow represents contribution of this thesis) 
The "Structure Functions Approach" (Appendix 2.3, p.82) [Lindsey 76] starts from a different point by investigating the role which Kolmogorov structure functions have in the theory of frequency stability. First, relations are established between PSD $S_{y}(f)$ and the Mth-order structure function $D_{b}^{(M)}(\tau)$ as well as the autocorrelation function $\boldsymbol{R}_{\Delta \varphi}(k r)$ of the differences of the phase. Then interconnections are established between the "Two-Sample Allan Variance", the "L-Sample Allan Variance" and the corresponding Structure Functions. In Appendix 2.3 we outline how different estimates of the variance $\hat{\sigma}_{i}^{2}$ can be interrelated to structure functions by using also the approach developed below.

It has been found [Barnes 71], [Rutman 78], [Barnes 66], [Allan 66] that the spectral density of the frequency fluctuations of any oscillator is mainly composed of five different kinds of noise and can be expressed by the following equation:

$$
S_{y}(f)=\sum_{j=-2}^{2} h_{j} f^{j}
$$

where the $h_{j}$ are constants. The most important of these noise terms are called random walk frequency noise $(j=-2)$, Alicker frequency noise $(j=-1)$ and white frequency noise $(j=0)$. We wish to develop a way of deriving different closedform expressions for each pers existing variance measures. Tris means that we are required to calculate the integral in eq.(2.12) and derive expressions as functions of $p, \tau, T_{1}$. In this way a translation between time and frequency domain measures is obtained. The resulting expressions can be used to predict not only the contribution of each noise term to the total oscillator noise, but also the way in which these terms depend on $p, r, T_{1}$. Last but not least, by using experimental estimates of $\hat{\sigma}_{i}^{2}$ versus $p, \tau$ and $T_{1}$, one can even predict which of all the possible noise terms is the most significant for a particular oscillator. 
The first successful attempt to derive closed-form expressions has been made by Allan [Allan 66] and Barnes [Barnes 66], [Barnes 71]. Their method is rather straightforward provided that the autocorrelation function $\boldsymbol{R}_{\varphi}(\tau)$ of the phase noise is known, or better still, if the function $U(\tau)$, as shown below, is known:

$$
U(\tau)=2\left[R_{\varphi}(0)-R_{\varphi}(\tau)\right]
$$

Moreover, their approach needs an expression of the sample variance such as [Barnes 71, eq.(10)], [Rutman 74, eq.(4.13)]:

$$
\sigma_{y}^{2}\left(\tau, p, T_{1}\right)=\frac{1}{p-1} \sum_{n=1}^{p}\left[\bar{y}_{\tau}(n)-\frac{1}{p} \sum_{k=1}^{p} \bar{y}_{\tau}(k)\right]^{2}
$$

which is the same eq.(2.14). Expected values of such an expression must be evaluated [Barnes 71, Appendix I] to obtain the variance estimate as a closedform integral equation [Barnes 71], eq.23]. Our approach to obtaining closed-form expressions is more elaborate but it exhibits the following advantages:

(a) It is a unified structured systematic approach applicable to any variance measure for any kind of noise. Hence "flicker frequency noise" does not need any special treatment.

(b) Calculation of variance expressions, such as the two-sample variance, does not require knowledge of $\boldsymbol{R}_{\varphi}(\tau)$ or $U(\tau)$. Instead, variances are expressed as integrals, such as eq.(2.12), involving the spectral density of frequency fluc. tuations, which defines the noise process considered. Moreover, the integral form for variances emphasizes the smoothing of the spectral density by the numerical filter associated with the considered time series. For that purpose, autocorrelation functions are generally useless. 
(c) If the powerful "transfer function approach" ([Rutman 74], [Rutman 78], [Boileau 78]) is followed according to which the shape of $G_{i}(f)$ is chosen a priori, then our method is more effective because, in contrast to the AllanBarnes approach, an expression of the sample variance, such as in eq.(2.42) is not even needed.

\subsubsection{Formulation and General Rule}

From eqs.(2.40) and (2.6), for different kinds of noise we have

$$
S_{j}(f) \triangleq S_{\bar{v}_{r}(n)}(f j)=h_{j} f^{j}\left(\frac{\sin \pi f \tau}{\pi f \tau}\right)^{2}
$$

Using eq.(2.12) we define the generalized variance estimate as

$$
\hat{\sigma}_{i, j}^{2}\left(\tau, p, T_{1}\right) \triangleq K_{i} \int_{0}^{\infty}\left|G_{i}(f)\right|^{2} S_{i}(f) d f
$$

In this expression

- $i$ inciicates the different variance measures, for example, $i=1$ denotes the Allan-Barnes variance, while $i=2$ indicates the Boileau measure.

- $j$ is a parameter indicating the type of noise, for example, $j=-1$ is used for the "flicker frequency noise".

Defining

$$
x=\pi f \tau, \quad \alpha(\pi f \tau) \triangleq \sin ^{2}(\pi r f \tau)
$$


then, for compact notation, we put $A \triangleq r x$ so that

$$
\alpha(x) \triangleq \sin ^{2} A
$$

Since step-wise functions are used to define a sample of the different variance measures, all corresponding filter terms appear as functions of $\sin ^{2} p A$. This can be easily seen by the filter expressions $\left|G_{1}(f)\right|^{2},\left|G_{2}(f)\right|^{2}$ (eqs.(2.28), (2.33)) as well as by the following two equations [Rutman 78]:

Hadamard:

$$
\left|G_{3}(f)\right|^{2}=\left(\frac{\sin p \pi f T_{1}}{\cos \pi f T_{1}}\right)^{2}, K_{3}=1
$$

Binomial Coefficient:

$$
\mid G_{4}(f) !^{2}=2^{2(p-1)} \sin ^{2(p-1)} \pi f T_{1}, K_{4}=1
$$

But from trigonometry we know that $\sin p A$ can always be written as

$$
\sin p A=\sin A \sum_{k} \rho_{k} \sin ^{2 k} A
$$

where $\rho_{k}$ is a set of constants. From eq.( $(2.48)$ and the previous statement it can be concluded that

$$
\left|G_{i}(x)\right|^{2}=\sum_{k} Z_{k, i} \alpha^{k}(x)
$$

where $Z_{k, i}$ is another set of constants related to $\rho_{k}$ and depending on the kind of the filter (subscript i). Equation (2.49) simply states that any filter term can be 
expressed as a summation of powers of the $\sin ^{2} A$ term with suitable coefficients. By using (2.6), (2.45) and (2.49), eq.(2.44) gives:

$$
\begin{aligned}
\hat{\sigma}_{i, j}^{2} & =\frac{K_{i} h_{j}}{(\pi \tau)^{j+1}} \int_{0}^{\infty} x^{j-2} \sin ^{2} x \sum_{k=1}^{p-1} Z_{k, i} \alpha^{k}(x) d x \\
& =\frac{K_{i} h_{j}}{(\pi \tau)^{j+1}} \sum_{k=1}^{p-1} Z_{k, i} \int_{0}^{\infty} x^{j-2} \sin ^{2} x \sin ^{2 k}(r x) d x
\end{aligned}
$$

or equivalently,

$$
\begin{gathered}
\hat{\sigma}_{i, j}^{2}=\frac{K_{i} h_{j}}{(\pi \tau)^{j+1}} \sum_{k=1}^{p-1} Z_{k, i} I_{k, j} \\
I_{k, j} \triangleq \int_{0}^{\infty} x^{j-2} \sin ^{2} x \sin ^{2 k}(r x) d x
\end{gathered}
$$

Equation (2.51a) is of great importance and it constitutes the general rule according to which closed-form variance equations can be derived. From this equation two important facts can be deduced:

(a) For the same type of noise, no matter which variance measure we wish to derive, the integral factor $I_{k}$ remains the same. Hence when considering different types of noise, only $I_{k}$ need be changed.

(b) When the type of variance measure changes, it only affects the summation for factor $Z_{k}$ and not the integral $I_{k}$.

Now let us proceed with the calculation of the integral $I_{k}$. By using the following trigonometric identity we can find a general form for the integral $I_{k}$ : 


$$
\sin ^{2 n-1} A=\frac{(-1)^{n-1}}{2^{2 n-2}} \sum_{j=0}^{n-1}\left(\begin{array}{c}
2 n-1 \\
j
\end{array}\right)(-1)^{j} \cdot \sin (2 n-1-2 j) A
$$

We have:

$$
\begin{aligned}
\sin ^{2 k}(r x) & =\sin (r x) \sin ^{2 k-1}(r x) \\
& =\frac{(-1)^{k-1}}{2^{2 k-2}} \sum_{j=0}^{k-1}\left(\begin{array}{c}
2 k-1 \\
j
\end{array}\right)(-1)^{j} \cdot \sin (r x) \sin ((2 k-1-2 j) r x) \\
& =\frac{(-1)^{k-1}}{2^{2 k-1}}\{[\cos (2 k-2) r x-\cos 2 k r x] \\
& -\left(\begin{array}{c}
2 k-1 \\
1
\end{array}\right)[\cos (2 k-4) r x-\cos (2 k-2) r x] \\
& +\left(\begin{array}{c}
2 k-1 \\
2
\end{array}\right) \cdot[\cos (2 k-6) r x-\cos (2 k-4) r x] \\
& \left.+\cdots+(-1)^{k-1}\left(\begin{array}{c}
2 k-1 \\
k-1
\end{array}\right)[1-\cos 2 r x]\right\}
\end{aligned}
$$

Hence the integral $I_{k, j}$ from (2.51b can be written as follows:

$$
I_{k, j}=\frac{(-1)^{k-1}}{2^{2 k-1}}-\sum_{m=0}^{k-1}(-1)^{m}\left(\begin{array}{c}
2 k-1 \\
m
\end{array}\right) L_{j}(k-m)
$$

where

$$
L_{j}(s) \triangleq \int_{0}^{\infty} x^{j-2} \sin ^{2} x[\cos (2 r(s-1) x)-\cos (2 r s x)] d x
$$


Hence one needs only to calculate $L_{j}(s)$ for a particular value of $j$ in order to be able to calculate the integral $I_{k}$. This means that it is actually possible to calculate all integrals a priori for any type of noise (for all values of $j$ ) and these values will always be the same no matter which variance is to be estimated. Then only $Z_{k}$ has to be calculated to get the different variance estimators.

Before proceeding to particular applications of this general theory it should be emphasized again that our method is based on direct integration and therefore expressions for autocorrelation functions of phase and frequency instabilities are not needed. Hence it can be foreseen that the method will be particularly useful when the "transfer function approach" of Rutman and Boileau is employed, according to which the integral expressions of the variance estimate are chosen a priori. Using our method, these integrals can be evaluated without the need to calculate the corresponding sample of the variance. This is because, according to the general rule (eq.(2.51a)), the variance estimate contains two terms $\left(Z_{k, i}, I_{k, j}\right)$, independent from one another.

\subsubsection{Applications}

First we will show that eq.(2.49) is true for $\left|G_{1}(x)\right|^{2},\left|G_{2}(x)\right|^{2}$, and then we will derive the Allan-Barnes variance estimator for "flicker" and "random walk" frequency noises.

(a1) Allan-Barnes Estimator

Assume $p=2 n-1=$ odd number (same procedure for even numbers). From [Mangulis 65] we have:

$$
\sin (2 n-1) A=(2 n-1) \sin A\left[1+\sum_{k=1}^{n-1} R_{k} \sin ^{2 k} A\right]
$$


where

$R_{k} \triangleq(-1)^{k}\left[(2 n-1)^{2}-1^{2}\right]\left[(2 n-1)^{2}-3^{2}\right] \cdots$
$k \leq n-1$

and

$$
R_{k}=0 \quad \text { for } \quad k \geq n
$$

Using (2.45), (2.56) and (2.58) and after some algebraic manipulations, eq.(2.28) gives:

$$
\begin{aligned}
\left|G_{1}(x)\right|^{2} & =-\left(\sum_{k=1}^{n-1} R_{k} \sin ^{2 k} A\right)^{2}-2 \sum_{k=1}^{n-1} R_{k} \sin ^{2 k} A \\
& =-\left(\sum_{k=1}^{n-1} R_{k} \alpha^{k}(x)\right)^{2}-2 \sum_{k=1}^{n-1} R_{k} \alpha^{k}(x)
\end{aligned}
$$

and by omitting the argument $x$ for simplicity:

$$
\begin{aligned}
\left|G_{1}\right|^{2} & =-\left(R_{1} \alpha+R_{2} \alpha^{2}+R_{3} \alpha^{3}+\cdots R_{n-1} \alpha^{n-1}\right)^{2} \\
& -2\left(R_{1} \alpha+R_{2} \alpha^{2}+R_{3} \alpha^{3}+\cdots+R_{n-1} \alpha^{n-1}\right) \\
& =-2 R_{1} \alpha-\alpha^{2}\left(R_{1}^{2}+2 R_{2}\right)-\alpha^{3}\left(2 R_{1} R_{2}+2 R_{3}\right)
\end{aligned}
$$




$$
-\alpha^{4}\left(2 R_{4}+2 R_{1} R_{3}+R_{2}^{2}\right) \cdots-\alpha^{k}\left(\sum_{i=0}^{k} R_{i} R_{k-i}\right)
$$

or equivalently:

$$
\left|G_{1}(x)\right|^{2}=\sum_{k=1}^{2 n-2} Z_{k, 1} \alpha^{k}(x)
$$

where

$$
Z_{k, 1} \triangleq-\sum_{i=0}^{k} R_{i} R_{k-i}
$$

(a2) Boileau-Picinbono Estimator

By using again the assumption of $p$ being an odd number, eq.(2.33) gives the following:

$$
\left|G_{2}(x)\right|^{2}=\left(\sum_{k=1}^{n-1} R_{k} \alpha^{k}(x)\right)^{2}
$$

or equivalently

$$
\begin{aligned}
\left|G_{2}(x)\right|^{2} & =\sum_{k=2}^{2 n-2} Z_{k, 2} \alpha^{k}(x) \\
Z_{k, 2} & \triangleq \sum_{i=1}^{k-1} R_{i} R_{k-i}
\end{aligned}
$$

As one can see, eqs.(2.60) and (2.63) for the filter terms in the above examples are quite similar, the only difference being the summation limits. 
(b1) Allan-Barnes Variance for "Flicker" Frequency Noise

We set $i=1, j=-1$. The calculation then proceeds in three steps as follows:

Step 1: Calculation of Integral $I_{k-1}$

For this kind of noise it is shown in Appendix 2-1 that

$$
L_{-1}(s)=-\frac{1}{2}[Q(s)-Q(s-1)]
$$

where $Q(s)$ is given by

$$
Q(s) \triangleq 2(s r)^{2} \ln (s r)-(s r+1)^{2} \ln (s r+1)-(s r-1)^{2} \ln |s r-1| \cdot
$$

Using (2.65), the integral (2.54) becomes

$$
I_{k,-1}=\frac{(-1)^{k-2}}{2^{2 k}}\left(Q(k)+\sum_{m=1}^{k-1}(-1)^{m} \cdot\left[\left(\begin{array}{c}
2 k-1 \\
m-1
\end{array}\right)+\left(\begin{array}{c}
2 k-1 \\
m
\end{array}\right)\right] Q(k-m)\right)
$$

From combinatorics

$$
\left(\begin{array}{c}
2 k-1 \\
m-1
\end{array}\right)+\left(\begin{array}{c}
2 k-1 \\
m
\end{array}\right)=\left(\begin{array}{c}
2 k \\
m
\end{array}\right)
$$

so that eq.(2.67) simplifies to

$$
I_{k,-1}=\frac{(-1)^{k-2}}{2^{2 k}} \sum_{m=0}^{k-1}(-1)^{m}\left(\begin{array}{c}
2 k \\
m
\end{array}\right) Q(k-m)
$$

This result is true for $k \leq 2 n-2=p-1$. 
Step 2: Calculation of Summation Term $Z_{k, 1}$

To calculate $Z_{k, 1}$ we only need to express $R_{k}$ as a combination. From the definition (2.57) it is easy to verify that

$$
R_{k}=\frac{(-1)^{k} 2^{2 k}}{2 k+1}\left(\begin{array}{c}
n-1+k \\
2 k
\end{array}\right)
$$

Now from eqs.(2.61), (2.70) and [Egorychev 84] we get

$$
\begin{aligned}
Z_{k, 1} & =-(-1)^{k} 2^{2 k} \sum_{i=0}^{k} \frac{1}{n+i}\left(\begin{array}{c}
n+i \\
2 i+1
\end{array}\right) \frac{1}{n+k-i} \cdot\left(\begin{array}{c}
n+k-i \\
2 k-2 i+1
\end{array}\right) \\
& =-(-1)^{k} 2^{2 k+1} \Phi_{k}
\end{aligned}
$$

where

$$
\Phi_{k} \triangleq \frac{1}{(2 n+k)(2 n-1)}\left(\begin{array}{c}
2 n+k \\
2 k+2
\end{array}\right)
$$

Step 3: Calculation of Variance $\hat{\sigma}_{1,-1}^{2}$

Using (2.69), (2.71) and (2.72), (2.51a) becomes

$$
\hat{\sigma}_{1,-1}^{2}=-2 K_{1} h_{-1} \sum_{k=1}^{p-1} \Phi_{k} \sum_{m=0}^{k-1}(-1)^{m} \cdot\left(\begin{array}{c}
2 k \\
m
\end{array}\right) Q(k-m)
$$

By employing double summation manipulation (2.73) gives

$$
\hat{\sigma}_{1,-1}^{2}=-2 K_{1} h_{-1}\left\{\Phi_{1}\left(\begin{array}{c}
2.1 \\
0
\end{array}\right) Q(1)\right.
$$




$$
\begin{aligned}
+ & \Phi_{2}\left[\left(\begin{array}{c}
2.2 \\
0
\end{array}\right) Q(2)-\left(\begin{array}{c}
2.2 \\
1
\end{array}\right) Q(4)\right] \\
+ & \Phi_{3}\left[\left(\begin{array}{c}
2.3 \\
0
\end{array}\right) Q(3)-\left(\begin{array}{c}
2.3 \\
1
\end{array}\right) Q(2)+\left(\begin{array}{c}
2.3 \\
2
\end{array}\right) Q(1)\right] \\
+ & \ldots\} \\
= & -2 K_{1} h_{-1} \sum_{k=1}^{p-1} Q(k) \cdot\left\{\left(\begin{array}{c}
2 . k \\
0
\end{array}\right) \Phi_{k}-\left(\begin{array}{c}
2(k+1) \\
1
\end{array}\right) \Phi_{k+1} \ldots\right. \\
& \left.\ldots+(-1)^{\ell}\left(\begin{array}{c}
2(k+\ell) \\
\ell
\end{array}\right) \Phi_{k+\ell}+\cdots\right\}
\end{aligned}
$$

Hence:

$$
\hat{\sigma}_{1,-1}^{2}=-2 K_{1} h_{-1} \sum_{k=1}^{p-1} Q(k) \cdot \sum_{\ell=0}^{2 n-k-2}(-1)^{\ell} \Phi_{k+\ell}\left(\begin{array}{c}
2 k+2 \ell \\
\ell^{\prime}
\end{array}\right)
$$

From (2.72), the second summation of (2.74) becomes

$$
\begin{aligned}
\sum_{\ell=0}^{2 n-k-2}(-1)^{\ell} \Phi_{k+\ell}\left(\begin{array}{c}
2 k+2 \ell \\
\ell
\end{array}\right)= & \frac{1}{2 n-1} \sum_{\ell=0}^{2 n-k-2}(-1)^{\ell} \frac{1}{2 n+k+\ell} \\
& \cdot\left(\begin{array}{c}
2 n+k+\ell \\
2 k+2 \ell+2
\end{array}\right)\left(\begin{array}{c}
2 k+2 \ell \\
\ell
\end{array}\right)
\end{aligned}
$$

But according to Appendix 2-2, we have

$$
\Psi \triangleq \sum_{\ell=0}^{2 n-k-2}(-1)^{\ell} \frac{1}{2 n+k+\ell}\left(\begin{array}{c}
2 n+k+\ell \\
2 k+2 \ell+2
\end{array}\right) \cdot\left(\begin{array}{c}
2 k+2 \ell \\
\ell
\end{array}\right)=\frac{p-k}{2 p}
$$


Hence (2.74) finally gives

$$
\hat{\sigma}_{1,-1}^{2}=-\frac{h_{-1}}{p(p-1)} \sum_{k=1}^{p-1}(p-k) Q(k)
$$

which is exactly the same as eq.(90) in [Barnes 71].

(b2) Allan-Barnes Variance for "Random Walk" Frequency Noise

To derive the Allan-Barnes variance measure for the characterization of random walk frequency noise, we set $i=1, j=-2$. The calculation requires two steps (the calculation of $Z_{k, 1}$ is not needed because it has already been done above).

Step 1: Calculation of the Integral $I_{k_{1}-2}$

It is shown in Appendix 2-1 that:

$$
\left.\begin{array}{l}
L_{-2}(s)=\pi r \\
L_{-2}(1)=\pi r-\frac{\pi}{3}
\end{array} \quad, s>1\right\}
$$

Hence the integral in (2.54) becomes

$$
\begin{aligned}
I_{k,-2} & =\pi r \frac{(-1)^{k-1}}{2^{2 k-1}} \sum_{m=0}^{k-2}(-1)^{m}\left(\begin{array}{c}
2 k-1 \\
m
\end{array}\right)+\pi r \frac{(-1)^{2 k-2}}{2^{2 k-1}} \cdot\left(\begin{array}{c}
2 k-1 \\
k-1
\end{array}\right) \\
& -\frac{(-1)^{2 k-2}}{2^{2 k-1}}\left(\begin{array}{c}
2 k-1 \\
k-1
\end{array}\right) \frac{\pi}{3}
\end{aligned}
$$

or

$$
I_{k,-2}=\pi r \frac{(-1)^{k-1}}{2^{2 k-1}} \sum_{m=0}^{k-1}(-1)^{m}\left(\begin{array}{c}
2 k-1 \\
m
\end{array}\right)-\frac{\pi}{3} \frac{(-1)^{2 k-2}}{2^{2 k-1}}\left(\begin{array}{c}
2 k-1 \\
k-1
\end{array}\right)
$$


Step 2: Calculation of the Variance $\hat{\sigma}_{1_{0}-2}^{2}$

$Z_{k}$ is the same for a particular variance estimator no matter what type of noise is used. Hence we do not need to calculate $Z_{k}$ again. Therefore from (2.71), (2.72) and (2.80) we have

$$
\left.\begin{array}{c}
\hat{\sigma}_{1,-2}^{2}=K_{1}\left(\sum_{\alpha}+\sum_{\beta}\right) \\
\Sigma_{\alpha} \triangleq 4 h_{-2}(\pi \tau) \sum_{k=1}^{p-1} \Phi_{k} \sum_{m=0}^{b-1}(-1)^{m}\left(\begin{array}{c}
2 k-1 \\
m
\end{array}\right)(\pi r) \\
\Sigma_{\beta} \triangleq \frac{1}{3} h_{-2} \pi(\pi \tau) \sum_{k=1}^{p-1} \Phi_{k}(-1)^{3 k-2}\left(\begin{array}{c}
2 k-1 \\
k-1
\end{array}\right)
\end{array}\right\}
$$

Applying the technique used to get (2.74) from (2.73) we obtain

$$
\Sigma_{\alpha}=4 h_{-2}(\pi \tau) \sum_{k=1}^{p-1} \pi r \cdot \sum_{\ell=0}^{2 n-k-2}(-1)^{\ell} \Phi_{k+\ell}\left(\begin{array}{c}
2 k+2 \ell-1 \\
\ell
\end{array}\right)
$$

It is shown in Appendix 2-2 that the inner summation of the above equation gives

$$
\sum_{\alpha, i n} \triangleq \sum_{\ell=0}^{2 n-k-2}(-1)^{\ell} \Phi_{k+\ell}\left(\begin{array}{c}
2 k+2 \ell-1 \\
\ell
\end{array}\right)=\frac{1}{4 p^{2}}(p-k)(p-k+1)
$$

so that (2.82) becomes

$$
\Sigma_{\alpha}=h_{-2} \frac{\tau(2 \pi)^{2} r(p-1)(p+1)}{12 p}
$$

In Appendix 2-2 exactly the same method used for calculating $\boldsymbol{\Sigma}_{\alpha, \text { in }}$ gives for $\Sigma_{\beta}$ 


$$
\Sigma_{\beta}=-h_{-2} \frac{4 \pi^{2} \tau(p-1)}{12 p}
$$

Using (2.84), (2.85), (2.81) finally gives

$$
\hat{\sigma}_{1,-2}^{2}=h_{-2} \frac{(2 \pi)^{2} \tau}{12}[r(p+1)-1]
$$

which is again equivalent to eq.(85) in [Barnes 71].

Concluding this section we observe that, according to the general rule formulated in eqs.(2.51a) and (b), the whole proress can be divided into the calculation of two independent terms: a summation term $Z_{k, i}$ which depends only on the variance measure and an integral term $I_{k, j}$ which depends only upon the type of noise to be characterized. As applications of our method, the Allan-Barnes variance was evaluated for "Flicker" and "random walk" frequency noises. From these derivations we can draw the conclusions listed below.

(a) No special treatment of the flicker noise case is needed because the new technique fully separates the "noise dependent" term $I_{k}$ from the "measurement processing dependent" term $Z_{k}$. In contrast, a special treatment of flicker noise would be needed if the conventional autocorrelation function approach were to be used.

(b) There is no need for an expression for the sample variance. By following this new method, the "transfer function approach" is rendered more effective, since the filter term $G_{i}(f)$ is chosen a priori in order to impose certain characteristics on the estimator. The sample of the corresponding variance measure which represents the sampling procedure of the counter, can be derived afterwards when the properties of the chosen estimator are considered satisfactory. 


\subsection{DESCRIPTION OF THE MEASUREMENT SYSTEM AND THE DEPENDANCE OF THE VARIANCE ESTIMATORS ON \\ $T_{1}, p, r$}

After the new generalized method for variance estimator expressions has been presented we need to see how these expressions can be used in frequency stability characterization. In order to be able to describe the effect of variations of $\tau, p$, $r, T_{1}$ on the variance estimates $\hat{\sigma}_{i}^{2}$ or, to put it in other terms, in order to justify the need to fix or vary some of these parameters such that their impact on the variance calculation can be observed, we first have to give a descisption of the physical measurement system. In this way we may avoid more abstraction concerning these parameters and demonstrate their physical substance such that we can see the interaction between time domain measures, stability characterization of hopping oscillators and specific hopping scenarios.

The measurement system is illustrated in Figure 2.6. A digital tuning command coming from microprocessor is applied to the input [a] of the DAC. A particular digital word, $d_{r}$ will cause the DAC to issue a corresponding analog voltage at point [b]. This voltage is applied to the input of the VCO which in turn produces a particular frequency $f_{r 1}$. The VCO remains at this particular frequency for a time interval which is called the dwell time $r$ of the frequency hopping system. In this system this is usually between 50 and $100 \mu$ sec. After this time has elapsed, the system changes the output frequency to $f_{\mathrm{r} 2}$, by means of another digital command word. It remains at the new frequency for another $\tau$ sec. Figure 2.7(a) is representative of the situation where we have a system which is continually hopping between two fixed frequencies $f_{r 1}$ and $f_{r 2}$. However, we can increase the number of frequencies to hundreds by using an appropriate sequence of digital words to control the DAC output. The output frequency of the VCO is now mixed with a 


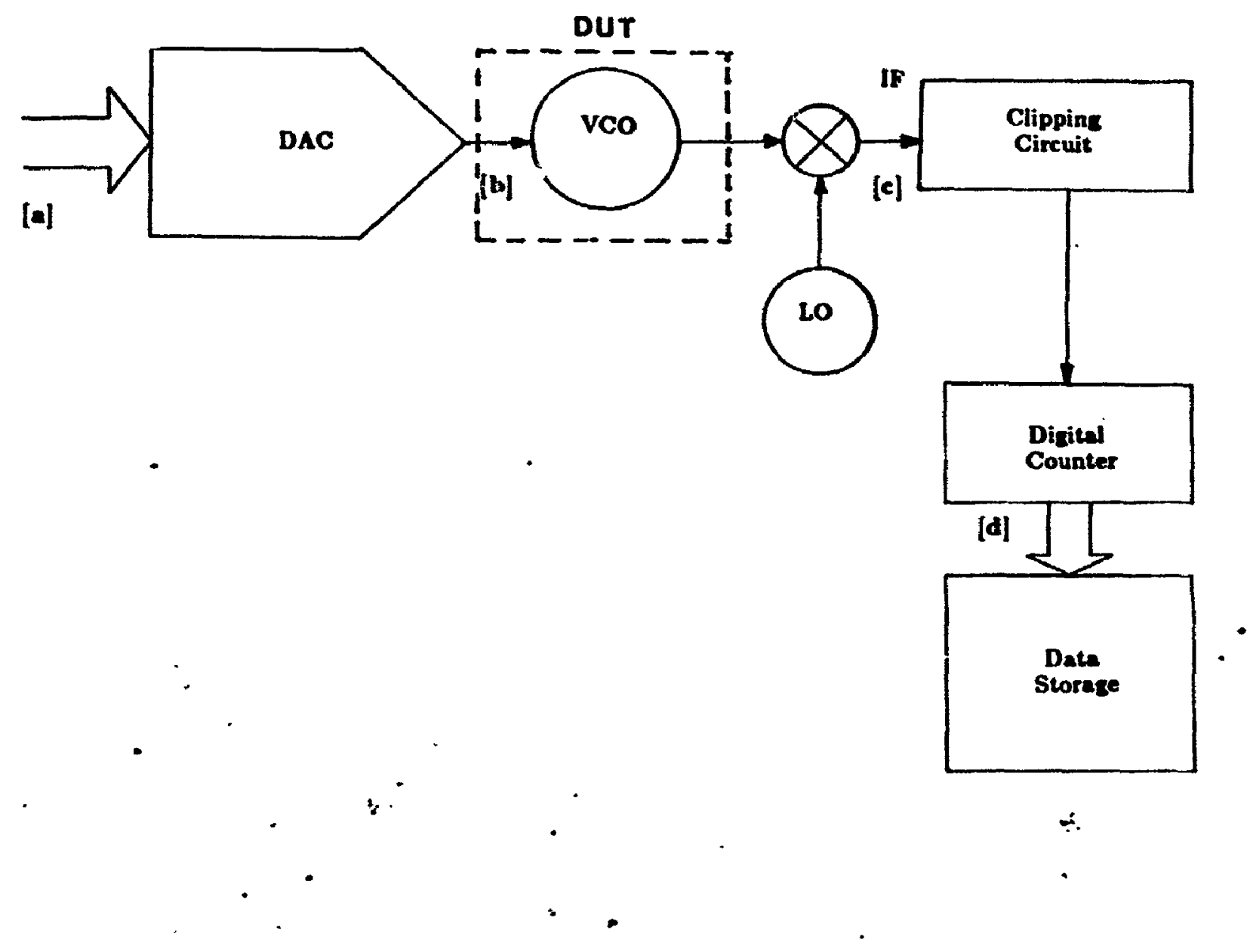

Figure 2.6: System implementation 

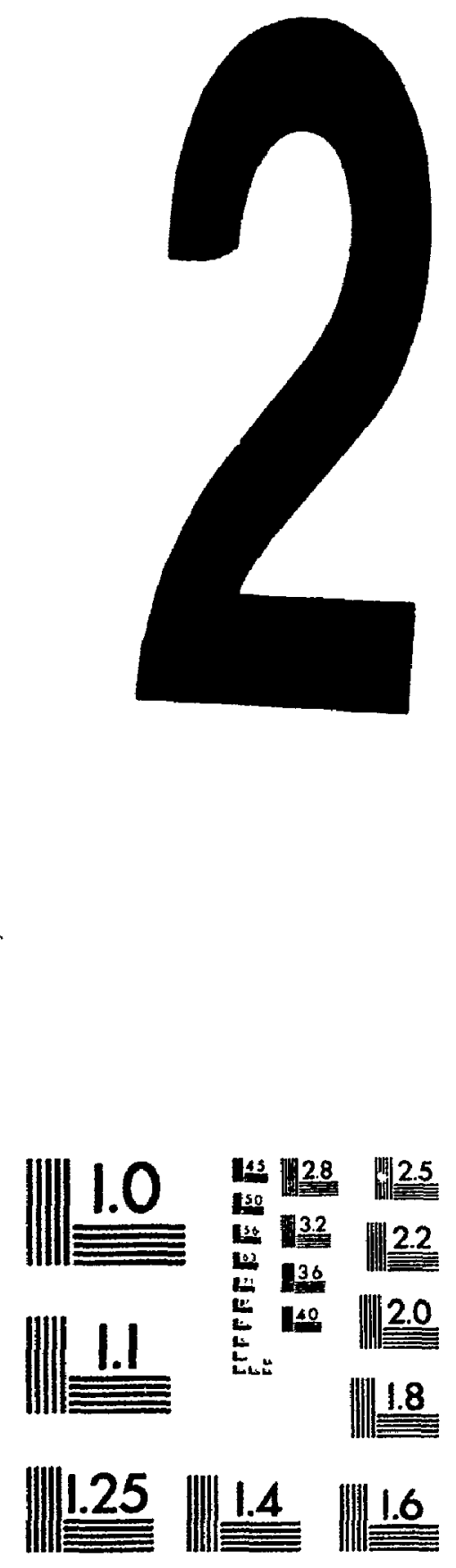

MICROCOPY RESOLUTION TEST CHART NATIONAL BUREAU OF STANDARDS STANDARD REFERENCE MATERIAL 1010 (ANSI and ISO TEST CHART No. 2) 

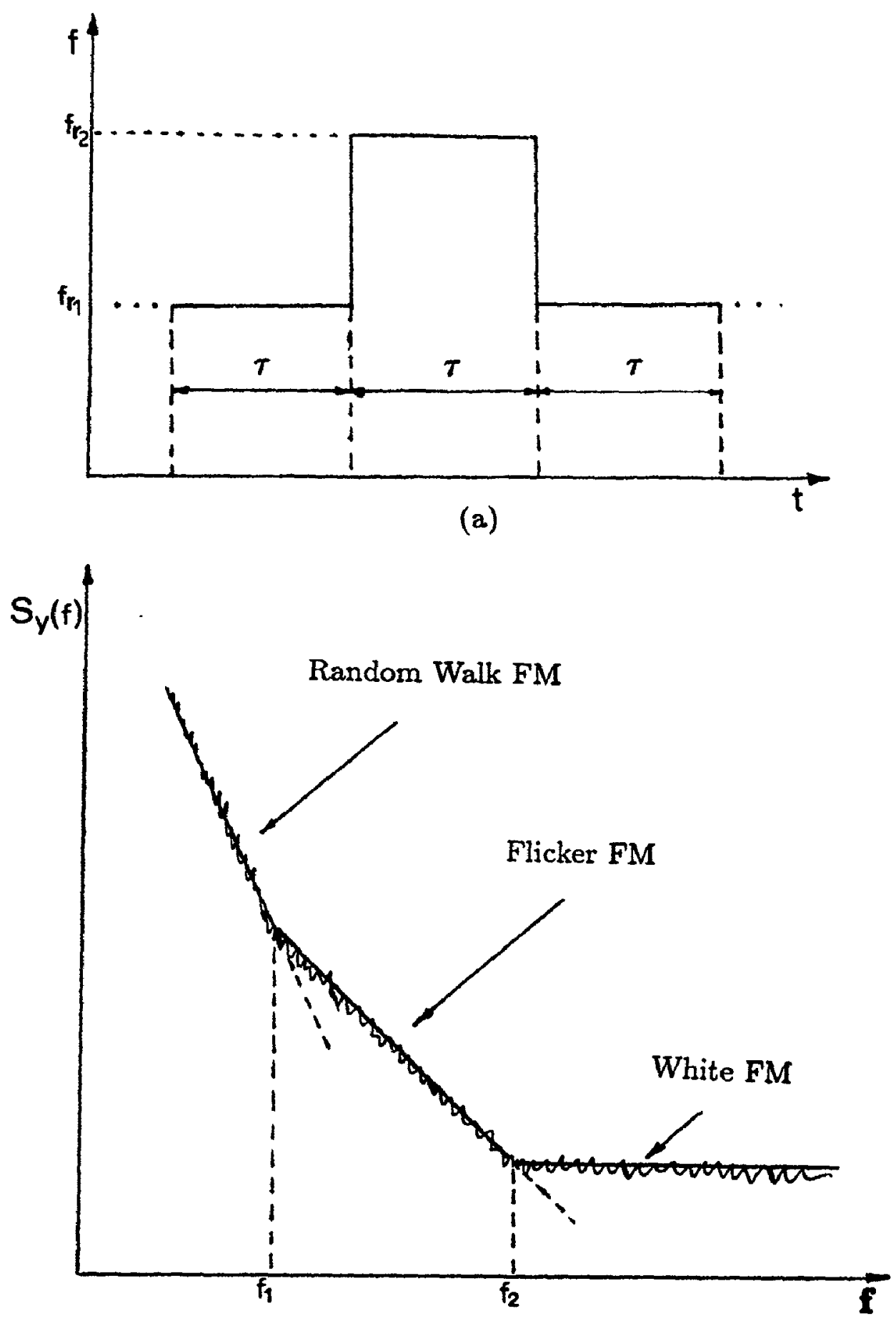

(b)

Figure 2.7: (a): System hopping between two frequencies; (b): typical spectrum of the frequency fluctuations 
very stable local oscillator (LO) frequency to produce a downconverted replica of the VCO output, which bears all of the STFS information [Barnes 71, Kartaschoff 78]. The mixing stage is needed because the frequencies are not directly counted within the VCO microwave operating range.

At point [c] of Figure 2.6, the IF signal is clipped and is then counted [d] for a period of $\tau$ secs every $T_{1}$ secs. (Figure 2.1(a)). The output of the digital counter is then stored. This output is actually given by $M$ in eq.(2.11). The key idea here is to have the system hopping and at the same time have the counter count during $\tau$ sec. Therefore the hopping rate is equal to $1 / \tau$. However, from the above it is obvious that we might not need to count every single VCO output frequency but once every $K$ frequencies. This constant $K$ will be directly related to $r=T_{1} / \tau$ because both of them have to do with the frequency data sampling rate.

The amount of data $(p)$ needed for $\hat{\sigma}_{i}^{2}$ characterization can also be determined by experimentation or theoretically from the closed form expressions, such that the variance exhibits a horizontal portion, as discussed in Section 2.3. Substituting $h_{-2}, h_{-1}$ given by (2.87) into eqs.(2.86), (2.77) for $\hat{\sigma}_{1,-2}^{2}, \hat{\sigma}_{1,-1}^{2}$, which were derived as applications of the generalized method in Section 2.4 , we get $(2.88,2.89)$ :

$$
\begin{aligned}
& \left.\begin{array}{c}
h_{-2}=S_{y}(f) f^{2} \\
h_{-1}=S_{y}(f) f
\end{array}\right\} \\
& \hat{\sigma}_{1,-2}^{2}=S_{y}(f) \cdot f^{2} \frac{(2 \pi)^{2} \tau}{12}[r(p+1)-1] \\
& \hat{\sigma}_{1,-1}^{2}=\frac{S_{y}(f) f}{p(p-1)} \sum_{n=1}^{p}(p-n)\left[-2(n r)^{2} \ln (n r)+(n r+1)^{2} \ln (n r+1)\right. \\
& \left.+(n r-1)^{2} \ln (n r-1)\right]
\end{aligned}
$$


Now if the $S_{y}(f)$ is given for example as in Figure 2.7(b) then we can apply values for $S_{y}$ in eq. $(2.88)\left(f<f_{1}\right)$ and get a value for $\hat{\sigma}_{1,-2}^{2}$ as a function of $\tau\left(\hat{\sigma}_{1,-2}^{2}(\tau)\right)$. Similarly for $f_{1}<f<f_{2}$ we apply a particular value to eq.(2.89) and calculate $\hat{\sigma}_{1,-1}^{2}$. Hence a resulting expression for $\hat{\sigma}$ would be:

$$
\hat{\sigma}_{1}^{2}=\hat{\sigma}_{1,-2}^{2}(\tau)+\hat{\sigma}_{1,-1}^{2}
$$

\subsection{SIGNIFICANCE AND APPLICABILITY OF TIME DOMAIN STABILITY MEASURES WITH RESPECT TO FREQUENCY DOMAIN MEASURES}

Comparing in principle Fourier transform (FT) methods with time domain (TD) variance measures it seems that FT are more general because no knowledge is needed about the spectrum $S_{y}(f)$ which is to be investigated. However for the reasons discussed below, variance analysis methods are more suitable for characterizing the stability of pulsed signal sources such as FHO. In the realm of frequency measurements, and among those who study frequency instability as a subject of its own, TD methods have had a wider applicability than FT techniques during the last 10 years. One reason for this is that frequency counters are easy to build and are required anyway for frequency measurements. Another reason is that variance measures are easy to compute, after frequency measurements have been collected. One need only look at the expressions (2.14) and (2.30), which give $\sigma_{1}^{2}$ and $\sigma_{2}^{2}$ respectively. Another advantage of TD techniques is that they make possible the measurement of noise at very low Fourier frequencies (i.e., below $1 \mathrm{~Hz}$ ). For the above reasons it is easily understood why TD measures and especially Allan-Barnes variance became increasingly importart in stability characterization in diverse fields 
of physics and communication electronics (lasers, masers, microwave radars).

On the other hand, FFT techniques require much more complex calculations and analysis. Quite sophisticated antialiasing filters have to be implemented to ensure low ripple in the passband and steep rolloff characteristics [Howe 81]. Furthermore leakage and picket-fence effects must be eliminated by choosing appropriate window functions.

On the contrary it became increasingly important to develop TD measures which would be suitable even for high resolution spectral analysis (i.e., Hadamard Variance). In this case, the more demands that are put on the compatabilities of these measures the more complicated computations they need. The above developments demonstrated the potential of TD methods for frequency stability characterization. As a result new measures were introduced in the last 7 years to enhance the capabilities of the old ones. One such measure is the modified Allan-Variance [Allan 81] which exhibits the capability to distinguish between white and flicker phase noise.

One last reason which particularly applies in our investigation is that since time domain adaptive algorithms are to be used for frequency correction of systematic drifts of the system, there should be a qualitative connection between the level of the time-domain variance and the error produced by the algorithm. 
Appendix 2.1

CALCULATION OF INTEGRAL TERMS OF THE FORM (2.55)

A. Calculation of $L_{-1}(s)$.

It will be shown that the following result is true:

$$
\begin{aligned}
L_{-1}(s) & =\int_{0}^{\infty} \frac{\sin ^{2} x[\cos 2 r(s-1) x-\cos 2 r s x]}{x^{3}} d x \\
& =-\frac{1}{2}[Q(s)-Q(s-1)]
\end{aligned}
$$

We will use Frullani's Theorem

$$
\int_{0}^{\infty} \frac{F(a x)-F(b x)}{x} d x=[F(0)-F(\infty)] \ln \frac{b}{a}
$$

$F(x)$ should be continuous. Here we introduce

$$
F(x)=\frac{\cos x-1}{x^{2}}
$$

By using L'Hospital's rule it is trivial to see that $F(0)=-1 / 2$, and that $F(\infty)=0$. $F(x)$ is also continuous. From trigonometry we have 


$$
\begin{aligned}
& \sin ^{2} x[\cos 2 r(s-1) x-\cos 2 r s x]=S_{1}+S_{2}+S_{3} \\
& S_{1} \triangleq 2 \cos (2 s r-2 r) x-2 \cos (2 s r) x \\
& S_{2} \triangleq \cos (2 s r+2) x-\cos (2 s r-2 r+2) x \\
& S_{3} \triangleq \cos (2 s r-2) x-\cos (2 s r-2 r-2) x
\end{aligned}
$$

Using simple algebraic manipulations

$$
\begin{aligned}
\frac{S_{1}}{x^{2}} & =2(2 s r)^{2}(F[(2 s r-2 r) x]-F[(2 s r) x]) \\
& +\left(8 r^{2}-16 s r^{2}\right) F[(2 s r-2 r) x] \\
\frac{S_{2}}{x^{2}} & =(2 s r+2)^{2}(F[(2 s r+2) x] \\
& -F[(2 s r-2 r+2) x]) \\
& +\left(8 s r^{2}+8 r-4 r^{2}\right) F[(2 s r-2 r+2) x] \\
\frac{S_{3}}{x^{2}} & =(2 s r-2)^{2}(F[(2 s r-2) x] \\
& -F[(2 s r-2 r-2) x]) \\
+ & \left(8 s r^{2}-8 r-4 r^{2}\right) F[(2 s r-2 r-2) x] .
\end{aligned}
$$


Adding and rearranging slightly we finally get

$$
\begin{aligned}
\int_{0}^{\infty} & \frac{\sin ^{2} x[\cos 2 r(s-1) x-\cos 2 r s x]}{x^{3}} d x \\
= & -\frac{1}{2}\left(2(s r)^{2} \ln \left[\frac{s r}{(s-1) r}\right]\right. \\
+ & (s r+1)^{2} \ln \left[\frac{(s-1) r+1}{s r+1}\right] \\
+ & (s r-1)^{2} \ln \left[\frac{(s-1) r-1}{s r-1}\right] \\
+ & r^{2} \ln \left[\frac{(s-1) r+1}{(s-1) r} \cdot \frac{(s-1) r-1}{(s-1) r}\right] \\
+ & 2 s r^{2} \ln \left[\frac{(s-1) r}{(s-1) r+1} \cdot \frac{(s-1) r}{(s-1) r-1}\right] \\
+ & \left.2 r \ln \left[\frac{(s-1) r-1}{(s-1) r+1}\right]\right) \cdot
\end{aligned}
$$

From (2.98), eq.( $(2.65)$ is derived as follows:

By defining,

$$
Q(s) \triangleq 2 s^{2} r^{2} \ln (s r)-(s r+1)^{2} \ln (s r+1)-(s r-1)^{2} \ln |s r-1|
$$

where 


$$
\left.\begin{array}{c}
Q_{1}(s) \triangleq 2 s^{2} r^{2} \ln (s r) \\
Q_{2}(s) \triangleq(s r+1)^{2} \ln (s r+1) \\
Q_{3}(s) \triangleq(s r-1)^{2} \ln |s r-1|
\end{array}\right\}
$$

we get:

$$
\begin{aligned}
& L_{-1}(s)=-\frac{1}{2}\left\{2 \ln \{(s-1) r\} \cdot\left[-s^{2} r^{2}-r^{2}+2 s r^{2}\right]+2 \ln \{s r\} s^{2} r^{2}\right. \\
& +\ln \{(s-1) r+1\} \cdot\left[r^{2}-2 s r^{2}-2 r+(s r+1)^{2}\right] \\
& -\ln \{s r+1\} \cdot[s r+1]^{2} \\
& +\ln \{(s-1) r-1\} \cdot\left[(s r-1)^{2}+r^{2}-2 s r^{2}+2 r\right] \\
& \left.-\ln \{s r-1\} \cdot(s r-1)^{2}\right\} \\
& =-\frac{1}{2}\left\{-2 \ln \{(s-1) r\}[s r-r]^{2} \leftarrow-Q_{1}(s-1)\right. \\
& +2 \ln (s r) \cdot s^{2} r^{2} \quad \leftarrow Q_{1}(s) \\
& +\ln \{(s-1) r+1\} \cdot[(s-1) r+1]^{2} \leftarrow-Q_{2}(s-1) \\
& -\ln \{s r+1\} \cdot[s r+1]^{2} \quad \leftarrow Q_{2}(s) \\
& +\ln \{(s-1) r-1\}[(s-1) r-1]^{2} \quad \leftarrow-Q_{3}(s-1) \\
& \left.-\ln \{s r-1\} \cdot(s r-1)^{2}\right\} \quad \leftarrow Q_{3}(s)
\end{aligned}
$$


and therefore

$$
L_{-1}(s)=-\frac{1}{2}\left\{Q_{1}(s)-Q_{1}(s-1)+Q_{2}(s)-Q_{2}(s-1)+Q_{3}(s)-Q_{3}(s-1)\right\}
$$

which gives:

$$
L_{-1}(s)=-\frac{1}{2}\{Q(s)-Q(s-1)\}
$$

where $Q(s)$ is defined in (2.66).

B. Calculation of $L_{-2}(s)$.

For $j=-2$ in (2.55) $L_{-2}(s)$ is equivalent to

$$
\begin{aligned}
L_{-2}(s) & =2 \int_{0}^{\infty} \frac{\sin ^{2} x \sin ^{2} r s x}{x^{4}} d x \\
& -2 \int_{0}^{\infty} \frac{\sin ^{2} x \sin ^{2} r(s-1) x}{x^{4}} d x
\end{aligned}
$$

By using [De Haan p.220], (2.102) becomes

$$
L_{-2}(s)=\pi r
$$

Separately, by using the same reference we have

$$
L_{-2}(1)=2 \int_{0}^{\infty} \frac{\sin ^{2} x \sin ^{2} r x}{x^{4}} d x=\pi r-\frac{\pi}{3}
$$


Appendix 2.2

\section{CALCULATION OF SUMMATIONS OF BINOMIAL PRODUCTS}

The following binomial identities are repeatedly used throughout this Appendix:

$$
\begin{gathered}
\frac{1}{2 n+k+\ell}\left(\begin{array}{c}
2 n+k+\ell \\
2 k+2 \ell+2
\end{array}\right) \\
=\frac{1}{2 n-1}\left(\begin{array}{c}
2 n+k+\ell \\
2 k+2 \ell+2
\end{array}\right) \\
-\frac{1}{2(2 n-1)}\left(\begin{array}{c}
2 n+k+\ell-1 \\
2 k+2 \ell+1
\end{array}\right) \\
(-1)^{m}\left(\begin{array}{c}
n+m-1 \\
m
\end{array}\right)=\left(\begin{array}{c}
-n \\
m
\end{array}\right) \\
\sum_{\ell=0}^{N}\left(\begin{array}{c}
x+l z \\
\ell
\end{array}\right)\left(\begin{array}{c}
y-\ell z \\
N-\ell
\end{array}\right)=\sum_{\ell=0}^{N} z^{\ell}\left(\begin{array}{c}
x+y-\ell \\
N-\ell
\end{array}\right)
\end{gathered}
$$

Equations (2.106) and (2.107) are combinatorial identities found in [Gould 1972].

\section{A. Proof of (2.76)}

Using (2.105) and (2.106), we get for $\Psi$ 


$$
\begin{aligned}
\Psi & =\frac{1}{2 n-1} \sum_{\ell=0}^{2 n-k-2}\left(\begin{array}{c}
2 n+k+\ell \\
2 k+2 \ell+2
\end{array}\right)\left(\begin{array}{c}
-2 k-\ell-1 \\
\ell
\end{array}\right) \\
& -\frac{1}{2(2 n-1)} \sum_{\ell=0}^{2 n-k-2}\left(\begin{array}{c}
2 n+k+\ell-1 \\
2 k+2 \ell+1
\end{array}\right) \\
& \cdot\left(\begin{array}{c}
-2 k-\ell-1 \\
\ell
\end{array}\right)
\end{aligned}
$$

or equivalently

$$
\left.\begin{array}{l}
\Psi=\frac{1}{p} \Psi_{1}-\frac{1}{2 p} \Psi_{2} \\
\Psi_{1} \triangleq \sum_{\ell=0}^{2 n-k-2}\left(\begin{array}{c}
2 n+k+\ell \\
(2 n-k-2)-\ell
\end{array}\right)\left(\begin{array}{c}
-2 k-\ell-1 \\
\ell
\end{array}\right) \\
\Psi_{2} \triangleq \sum_{\ell=0}^{2 n-k-2}\left(\begin{array}{c}
2 n+k+\ell-1 \\
(2 n-k-2)-\ell
\end{array}\right)\left(\begin{array}{c}
-2 k-\ell-1 \\
\ell
\end{array}\right)
\end{array}\right\}
$$

Both $\Psi_{1}$, and $\Psi_{2}$ are of the form in (2.107).

Inserting $x=-2 k-1, y=2 n+k, z=-1$, and $N=2 n-k-2$ in (2.107) we get for $\Psi_{1}$

$$
\Psi_{1}=\sum_{\ell=0}^{N}(-1)^{i}(N+1-\ell)
$$

Similarly for $\Psi_{2}$, (the only difference is, $y=2 n+k-1$ ) we get

$$
\Psi_{2}=\sum_{\ell=0}^{N}(-1)^{\ell}
$$

For $\mathrm{iV}=$ even we have 


$$
\begin{aligned}
\Psi_{1}^{e v} & =(N+1) \sum_{\ell=0}^{N}(-1)^{\ell}-\sum_{\ell=0}^{N} \ell(-1)^{\ell} \\
& =N+1-\frac{N}{2}
\end{aligned}
$$

and

$$
\Psi_{2}^{e v}=1
$$

Hence, from (2.109), we have

$$
\Psi^{e v}=\frac{N+1}{2 p}=\frac{p-k}{2 p} .
$$

It can be similarly proved that $\Psi^{\text {odd }}$ is exactly the same.

\section{B. Calculation of $\sum_{\alpha . i n}$.}

Comparing (2.76) with (2.83) one sees that the expression for $\Sigma_{\alpha . i n}$ is very similar to that for $\Psi$. Hence, the evaluation of $\sum_{\alpha, i n}$ is quite easy by following exactly the same steps. Nevertheless, for mathematical rigor the detailed calculation is given below.

From (2.106)

$$
(-1)^{\ell}\left(\begin{array}{c}
2 k+2 \ell-1 \\
\ell
\end{array}\right)=\left(\begin{array}{c}
-2 k-\ell \\
\ell
\end{array}\right)
$$

and by using (2.105) we take 


$$
\left.\begin{array}{l}
\sum_{\alpha . \text { in }}=\frac{1}{p}\left(\Theta_{1}-\Theta_{2}\right) \\
\Theta_{1} \triangleq \sum_{\ell=0}^{N_{k}} \frac{1}{p}\left(\begin{array}{c}
2 n \therefore k+\ell \\
N_{k}-\ell
\end{array}\right)\left(\begin{array}{c}
-2 k-\ell \\
\ell
\end{array}\right) \\
\Theta_{2} \triangleq \sum_{\ell=0}^{N_{k}} \frac{1}{2 p}\left(\begin{array}{c}
2 n+k+\ell-1 \\
N_{k}-\ell
\end{array}\right)\left(\begin{array}{c}
-2 k-\ell \\
\ell
\end{array}\right)
\end{array}\right\}
$$

where $N_{k}=2 n-k-2$.

Both $\Theta_{1}$ and $\Theta_{2}$ are of the form in (2.107). Inserting $x=-2 k, y=2 n+k, z=$ -1 , we get for $\Theta_{1}$

$$
\begin{aligned}
\Theta_{1} & =\frac{1}{p} \sum_{\ell=0}^{N_{k}}(-1)^{\ell}\left(\begin{array}{c}
2 n-k-\ell \\
N_{k}-\ell
\end{array}\right) \\
& =\frac{1}{2 p} \sum_{\ell=0}^{N_{k}}(-1)^{\ell}\left(N_{k}+2-\ell\right)\left(N_{k}+1-\ell\right) .
\end{aligned}
$$

Similarly for $x=-2 k, y=2 n+k-1, z=-1$ we have

$$
\begin{aligned}
\Theta_{2} & =\frac{1}{2 p} \sum_{\ell=0}^{N_{k}}(-1)^{\ell}\left(\begin{array}{c}
2 n-k-1-\ell \\
N_{k}-\ell
\end{array}\right) \\
& =\frac{1}{2 p} \sum_{\ell=0}^{N_{k}}(-1)^{\ell}\left(N_{k}+1-\ell\right) .
\end{aligned}
$$

Hence, $\sum_{\alpha . i n}$ becomes 


$$
\left.\begin{array}{c}
\Sigma_{\alpha, \text { in }}=\frac{1}{p^{2}}\left[\left(N_{k}+1\right) \Sigma_{1}-\left(N_{k}+1\right) \Sigma_{2}+\Sigma_{3}\right] \\
\Sigma_{1} \triangleq \sum_{\ell=0}^{N_{k}}(-1)^{\ell}\left(N_{k}+1-\ell\right) \\
\Sigma_{2} \triangleq \sum_{\ell=0}^{N_{k}}(-1)^{\ell} \ell, \Sigma_{3} \triangleq \sum_{\ell=0}^{N_{k}}(-1)^{\ell} \ell^{2} .
\end{array}\right\}
$$

For $N_{k}=$ even and using (2.112) we get

$$
\Sigma_{1}=\frac{N_{k}+1}{2}, \quad \Sigma_{2}=\frac{N_{k}}{2}
$$

It can also be easily shown that

$$
\Sigma_{3}=\frac{N_{k}\left(N_{k}+1\right)}{2}
$$

(2.116) - (2.119) give

$$
\Sigma_{\alpha . \text { in }}^{e v}=\frac{1}{2 p^{2}} \frac{\left(N_{k}+1\right)\left(N_{k}+2\right)}{2}
$$

For $N_{k}$ odd the same result can be easily obtained.

\section{Calculation of $\Sigma_{\beta}$.}

From (2.81)

$$
\begin{aligned}
\Sigma_{\beta}= & \frac{4}{3} \frac{h_{-2}}{p} \pi(\pi \tau) \sum_{k=1}^{N_{\beta}} \frac{1}{2 n+k} \\
& \cdot\left(\begin{array}{c}
2 n+k \\
2 k+2
\end{array}\right)(-1)^{k}\left(\begin{array}{c}
2 k-1 \\
k
\end{array}\right)
\end{aligned}
$$


where $N_{\beta}=p-1$. By using (2.105) (with $\left.l=0\right)$, and (2.106) in

$$
\frac{1}{2 n+k}\left(\begin{array}{c}
2 n+k \\
2 k+2
\end{array}\right),(-1)^{k}\left(\begin{array}{c}
2 k-1 \\
k
\end{array}\right)
$$

we get, respectively

$$
\begin{aligned}
\Sigma_{\beta} & =h_{-2} \frac{4 \pi^{2} \tau}{3 p^{2}}\left[\sum_{k=1}^{N_{\beta}}\left(\begin{array}{c}
N_{\beta}+2+k \\
N_{\beta}-k
\end{array}\right)\left(\begin{array}{c}
-k \\
k
\end{array}\right)\right. \\
& \left.-\frac{1}{2} \sum_{k=1}^{N_{\beta}}\left(\begin{array}{c}
N_{\beta}+1+k \\
N_{\beta}-k
\end{array}\right)\left(\begin{array}{c}
-k \\
k
\end{array}\right)\right]
\end{aligned}
$$

and by using (2.107) again

$$
\begin{aligned}
\Sigma_{\beta} & =h_{-2} \frac{4 \pi^{2} \tau}{3 p^{2}}\left[\sum_{k=1}^{N_{\beta}}\left(\begin{array}{c}
N_{\beta}+2-k \\
N_{\beta}-k
\end{array}\right)(-1)^{k}\right. \\
& \left.-\frac{1}{2} \sum_{k=1}^{N_{\beta}}\left(\begin{array}{c}
N_{\beta}+\ell+k \\
N_{\beta}-k
\end{array}\right)(-1)^{k}\right]
\end{aligned}
$$

This easily gives

$$
\Sigma_{\beta}=-h_{-2} \frac{4 \pi^{2} \tau\left(N_{\beta}+1\right) N_{\beta}}{12 p^{2}}=-h_{-2} \frac{4 \pi^{2} \tau(p-1)}{12 p}
$$

which is what was to be proved (i.e., (85)). 


\section{RELATIONSHIP TO STRUCTURE FUNCTIONS}

Equations (2.51a) and (2.51b) give

$$
\hat{\sigma}_{i, j}^{2}=\frac{K_{j}}{(\pi \tau)^{j+1}} \sum_{k=1}^{p-1} z_{k, i} \int_{0}^{\infty}\left(h_{j} x^{j}\right) \frac{\sin ^{2} x \sin ^{2 k}(r x)}{x^{2}} d x
$$

From (2.56) and (2.52) we get

$$
\begin{aligned}
\sin ^{2 k}(r x) & =\sin ^{2 k-1}(r x) \sin (r x) \\
& =\frac{(-1)^{k-1}}{2^{2 k-2}}(\sin (2 k-1) r x \\
& -\left(\begin{array}{c}
2 k-1 \\
1
\end{array}\right) \sin (2 k-3) r x \\
& \left.+\cdots+(-1)^{k-1}\left(\begin{array}{c}
2 k-1 \\
k-1
\end{array}\right) \sin r x\right) \\
& \cdot r \sin x\left(1+\sum_{k_{0}=1}^{(r-1) / 2} R_{k_{0}} \sin ^{2 k_{0}} x\right) .
\end{aligned}
$$

Again using (2.56) to analyze each particular term of the form $\sin (2 k-j) r x$ in the above equation, it can be easily seen that $\sin ^{2 k} r x$ can finally be expressed as a summation of $\sin ^{2 M} x$ terms with different coefficients.

Hence, the integral in (2.125) will be a summation of integrals as the one below 


$$
\int_{0}^{\infty}\left(h_{j} x^{j}\right) \frac{\sin ^{2 M} x}{x^{2}} d x
$$

Having in mind that $h_{j} x^{j}$ corresponds to the Power Spectral Density $S_{\psi}(\omega)$ (according to Lindsey's notation in [Lindsey 76]) and that $x=\omega \tau / 2$ (see eq.(2.46)), it is easy to see that integrals like (2.127) corresponding to $M$ th order structure functions $D_{\psi}^{(M)}(\tau)\left((1.8)\right.$ in [Lindsey 76]) with autocorrelation function $R_{\Delta \psi}(k \tau)$ given by (1.6) in [Lindsey 76]

$$
\begin{gathered}
D_{\psi}^{(M}(\tau)=2^{2 M} \int_{-\infty}^{\infty} \sin ^{2 M}\left(\frac{\omega \tau}{2}\right) \frac{S_{\psi}(\omega)}{\omega^{2}} \frac{d \omega}{2 \pi} \\
R_{\Delta \psi}(k \tau)=4 \int_{-\infty}^{\infty} e^{-j k \omega \tau} \frac{S_{\psi}(\omega)}{\omega^{2}} \sin ^{2}\left(\frac{\omega \tau}{2}\right) \frac{d \omega}{2 \pi}
\end{gathered}
$$

Hence, there is always a relationship between structure functions and variance estimators. 


\section{Chapter 3}

\section{DEVELOPMENT OF THE MCMH SYNTHESIZER AND ITS MAIN CHARACTERISTICS}

\subsection{INTRODUCTION}

In the second chapter a general approach was developed for the characterization of the short-term noise of frequency hopping oscillators (FHOs). Together with that, a measurement system was described which can be used to obtain the quantitative noise behaviour of FHOs. It will be demonstrated here that by incorporating a microprocessor $(\mu \mathrm{P})$ to perform processing and control operations in the measurement system, we obtain a complete microwave frequency synthesizer system which we call a Microprocessor Controlled Microwave Hopping (MCMH) synthesizer. This is based on the idea that the frequency counter can be used both as a means of obtaining data for stability characterization and as a feedback element, in the sense that it continuously verifies the expected output from the VCO, which can be potentially corrected for possible offsets through the $\mu \mathrm{P}$.

This synthesis technique can be regarded as an application of the previously- 
described noise characterization procedure for hopping oscillators. These synthesizers for example could be applied to hybrid DS/FH spread spectrum systems. Hence in Section 3.2 of this chapter, the general requirements of such a spread spectrum synthesizer will be presented with the intention to desiga, implement and measure such a system in Chapter 5. In Section 3.3 the particular design will be given in more detail and its most important features will be discussed further. Section 3.4 is devoted on the function of the counter in the actual system and its equivalent "black box" characterization. Section 3.5 investigates the principle of applying adaptive time domain techniques to prevent the system from departing from its nominal frequency pattern assignment due to systematic drifts. Then, the design criteria and flexibility of implementation such as MCMH will be shown. Finally, a comparison will be made with other frequency hopping synthesis methods.

\subsection{REQUIREMENTS OF A HOPPING SYNTHESIZER EMPLOYED IN A SPREAD-SPECTRUM COMMUNICATION SYSTEM}

Assuming a real situation where there exists a transmitter (TX), a receiver (RX) and a hostile jammer located off the straight TX-RX line, it is reasonable to anticipate that the TX to hostile-jammer to RX path is at least $20 \mathrm{~km}$ longer than the direct TX-RX line in order to avoid follower jammers. This means that the direct signal arrives at least $67.6 \mu \mathrm{sec}$ before the reflected one. This consideration suggests a frequency-hopping system with a potential for a minimum hopping rate equal to $15.0 \mathrm{kh} / \mathrm{s}$. This rate will guarantee that the system is located at another frequency before the reflected signal could be received via the hostile jammer.

Since the purpose of this section is to demonstrate a representative system for 
SS application, the RF bandwidth of the system is not considered fixed. However for purposes of convenience we assume a required $\mathrm{RF}$ bandwidth of $500 \mathrm{MHz}$, which is a typical figure in an actual system whose centre frequency is located in the 1-2 GHz region [Drapac 87, Raines 86].

As has been discussed in the examples of DS and FH systems in Chapter 1 before, neither DS nor FH alone can be used effectively for a $500 \mathrm{MHz}$ bandwidth. DS requires a code rate of $250 \mathrm{Mb} / \mathrm{s}$ which is not easily obtained. On the other hand, FH needs 8,333 chip frequencies separated by $60 \mathrm{KHz}$ to transmit with an information rate of $10 \mathrm{~kb} / \mathrm{s}$ at three frequency chips per bit. This number of frequencies in conjunction with the required hopping rate $(30 \mathrm{kh} / \mathrm{s})$ is not easily implemented. ${ }^{1}$ If the hopping rate were to be reduced, the number of frequency channels would be increased. However as given in the example of hybrid systems, a DS/FH system is easily feasible with a $500 \mathrm{~kb} / \mathrm{s}$ clock rate and a frequency-hopping synthesizer with 500 frequencies, $1 \mathrm{MHz}$ apart, hopping at $15 \mathrm{kh} / \mathrm{s}$. This is the case when low-speed data have to be transmitted. For high-speed data transmission (of the order of 1 $\mathrm{Mb} / \mathrm{s}$ ) the information signal itself produces the spreading effect. Hence the same synthesizer hopping at $15 \mathrm{kh} / \mathrm{s}$ can be used without any direct sequencing. For a centre frequency of $1 \mathrm{GHz}$, a signal deviation between $0.01 \%$ ( 1 part in $10^{4}$ ) and $0.001 \%$ ( 1 part in $10^{4}$ ) is considered acceptable. The $0.01 \%$ figure corresponds to a deviation of $100 \mathrm{KHz}$ for a nominal $1 \mathrm{GHz}$ signal while $0.001 \%$ corresponds to 10 $\mathrm{KHz}$ for the same nominal signal. Depending on the value of the modulation sensitivity (MS) of a particular VCO, the acceptable frequency resolution corresponds to a specific VCO tuning voltage $\left(V_{t}\right)$ accuracy. For example if a VCO is to cover a $500 \mathrm{MHz}$ band with a MS equai to $50 \mathrm{MHz} / \mathrm{V}$ it needs a 10-Volt range. For a signal deviation of $0.001 \%(10 \mathrm{KHz})$ the $V_{t}$ deviation would be $200 \mu \mathrm{V}\left(\frac{1 V}{50 M H z} \times 10 K H z\right)$. Hence the required $V_{t}$ accuracy is $0.002 \%$.

\footnotetext{
${ }^{1}$ However some manufacturers (e.g., Comstron) provide synthesizers with 50,000 frequencies over $2 \mathbf{G H z}$ bands.
} 
Table 3.1: SYSTEM SPECIFICATIONS

\begin{tabular}{lr}
\hline 1. RF bandwidth & $\geq 500 \mathrm{MHz}$ \\
2. Signal deviation & 1 part in $10^{4}$ to 1 part in $10^{5}$ \\
3. Tuning voltage accuracy & $0.01 \%$ to $0.002 \%$ of F.S. \\
4. Settling time & ts $\leq 6.66 \mu \mathrm{sec}$ \\
5. Frequency resolution* & $100 \mathrm{KHz}$ to $10 \mathrm{KHz}$ \\
6. Hopping rate & $\geq 15 \mathrm{kh} / \mathrm{s}$ \\
7. Dwell period & $66.7 \mu \mathrm{sec}$ \\
8. Output power & $+10 \mathrm{dBm}$ \\
9. SSB Phase Noise (10 KHz away from carrier) & $-80 \mathrm{dBc} / \mathrm{Hz}$ \\
\hline
\end{tabular}

* Related to how many frequencies can be generated and how accurately a frequency can be set.

As far as frequency stability specification is concerned it is reasonable to accept a single sideband phase noise of $-80 \mathrm{dBc} / \mathrm{Hz}$ at $10 \mathrm{KHz}$ away from carrier. This is a representative figure for most modern frequency synthesizers (phase-locked and direct) [Kadpal 88], [Wolfson 89], [Dodson 81]. The required settling time of the system is usually determined frem the hopping rate by employing a design rule requiring that the system settle within $10 \%$ of its dwell time. For a $15 \mathrm{kh} / \mathrm{s}$ rate (and therefore a dwell time of $66.66 \mu \mathrm{sec}$ ) the synthesizer must settle within 6.66 $\mu$ sec. In Table 3.1 above, the specifications of an operational system based on the above discussion are indicated.

\subsection{FRFQUENCY SYNTHESIS BY A MICRO- PROCESSOR CONTROLLED VCO}

Now that we have outlined some general specifications for a hopping synthesizer, we may proceed to describe the MCMH synthesizer in more detail. Figure 3.1 depicts the complete system. One can see now that with the microprocessor it constitutes a complete frequency synthesis system. The $\mu \mathrm{P}$ receives as input the counts from the counter and issues two digital words: one applied as an input to the DAC to 


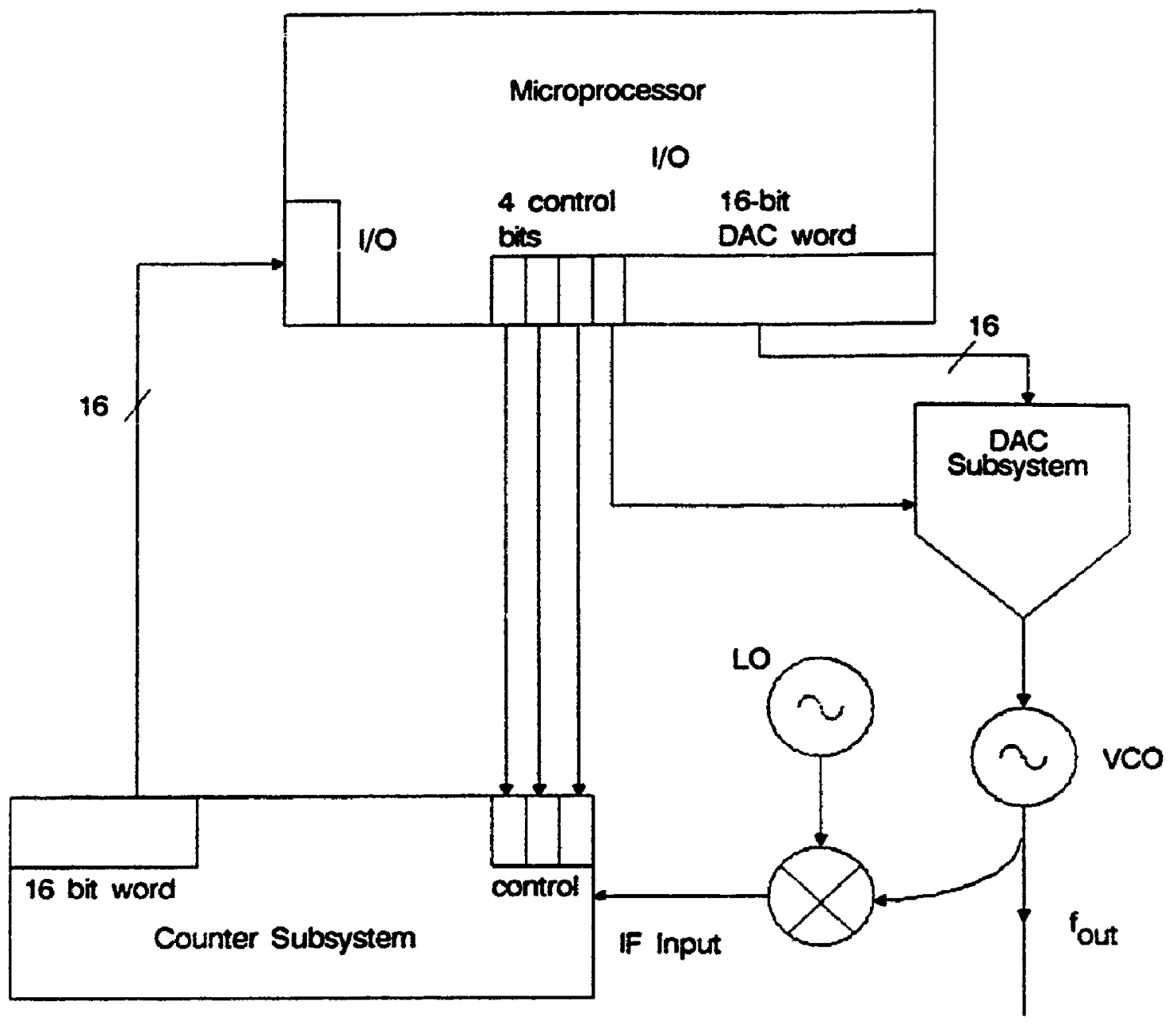

Figure 3.1: Block diagram of the MCMH synthesizer 
dictate the output frequency of the system and the other used as a control word to control the whole system. The $\mu \mathrm{P}$ is also used for either processing the time domain (TD) data to characterize the stability of the hopping system or to perform adaptive cancellation of frequency offsets. The processing for stability characterization can be done off-line while the adaptive frequency correction has to be performed on-line. When no real-time adaptive correction is done, the system does not use the feedback from the counter to adjust appropriately the output frequency and therefore it is said to be operating in the open-loop mode. However, when continuous adjustment takes place through an adaptive algorithm, we say that the system is operating in the closed-loop mode.

The VCO is the most important part of the system since it constitutes the frequency-generating source. This means that the quality of the frequencies generated by the synthesizer is directly related to the noise behaviour of the VCO. On the other hand the VCO inposes the primary restrictions on the design of the system in terms of tunable bandwidth and tuning speed. The tuning speed is determined not only by the VCO response but also by the DAC number of bits. The required accuracy and tuning bandwidth specifications usually dictate the number of bits (b) that the DAC should have, by the following relation:

$$
b \geq \log _{2}\left(\frac{\text { Tuning Bandwidth }}{\text { Frequency Accuracy }}\right)
$$

where $b$ should be an integer. For example, for a $500-\mathrm{MHz}$ bandwidth, and resolution of $10 \mathrm{KHz}$, a 16-bit DAC is needed. Present state-of-the-art indicates that the fastest DACs can tune within $1 \mu \sec$ for a full voltage step to useful resolution. The full voltage step is $\mathbf{1 0}$ to 15 Volts. Therefore a reasonable choice for VCO tuning characteristics would be to settle in less than $1 \mu \mathrm{sec}$ to $0.01 \%$ accuracy with a tunable bandwidth of $500 \mathrm{MHz}$. The full tuning voltage step should correspond to the full voltage step in the output of the DAC (10-15V). As will be seen in Chapter 
5, to meet these specifications for both VCO and DAC and to keep the noise of the system to a reasonably low level is not an easy task.

The output of the VCO is then downcoverted using a stable LO. This is done in order to be able to digitize the IF and count it. Therefore the LO should be located at the middle of the $500 \mathrm{MHz}$ band so as to divide it into two equal bands of $250 \mathrm{MHz}$ each. In this way the counter is only required to count up to $250 \mathrm{MHz}$.

The $\mu \mathrm{P}$ should be able to do the necessary processing within a dwell time period, so that, if needed, it can make the necessary adjustments every time a digital command is issued to the DAC. However this is the most stringent limit for the processing time because it is very likely that the $\mu \mathrm{P}$ does not need to do calculations for every frequency but for every $K$ frequencies ( $K$ being any integer between 10 and 100). However using the calculated error the output frequencies should be corrected. Therefore, a reasonably simple processing algorithm should be chosen such that all processing is performed within less than $100 \mu \sec$ when the system is continuously adjusting its output frequency. Summarizing, in order to implement an MCMH system as described in the previous two sections we need:

- A microwave VCO with tuning voltage range of 10 to $15 \mathrm{~V}$ and output bandwidth of $500 \mathrm{MHz}$. It should settle within $0.01 \%$ of the final value within 100 nsec.

- A 16-bit DAC tuning in full voltage step (10-15V) within $1 \mu \sec$ with an accuracy of $0.01 \%$ of the final value.

- A fast counter subsystem being able to count up to an IF of $250 \mathrm{MHz}$.

- A $\mu \mathrm{P}$ for data storage, processing and control being able to perform relatively simple processing tasks within 60-100 $\mu$ sec. 


\subsection{THE COUNTER AS A LINEAR SYSTEM AND ITS IMPORTANCE AS A CRITICAL ELEMENT IN THE SYSTEM}

In Chapter 2, the counter was described as an element of a measurement system needed to collect the TD data to be used for stability characterization. In the previous sections we saw that the counter is also the feedback element of the system, since based on recent counts and their processing the output frequency can be adjusted and always stay where it should be within a few KHz. This increased importance of the counter leads us to present in more detail its operation as a linear system and its enhanced abilities as a TD stability and spectral analysis tool. Several publications [Allan 75, Peregrino 76, Howe 81] devote their major parts to the analysis and demonstration of counter capabilities. Following [Peregrino 76] we can outline the operation of the counter as a linear system as follows.

Figure 3.2(a) shows the counter as a black box with an impulse response $h(t)$ as in Figure 2.2(c). Figure 3.2(b) shows a black box which is both a counter and a "function generator". We call it "function generator" because the output $G(t)$ is given by:

$$
G(t)=\sum_{i=0} \alpha_{i} \bar{y}_{i}
$$

where the $\alpha_{i}$ are weight factors. Hence choosing various sets $\left\{\alpha_{i}, i=1,2, \cdots\right\}$ one can generate different "functions" of ihe sequence of measurements $\bar{y}_{i}$. The variance is:

$$
E\left\{G^{2}(t)\right\}=\hat{\sigma}_{G}^{2}=\int_{-\infty}^{\infty}\left|H_{G}(f)\right|^{2} S_{\varphi}(f) d f
$$




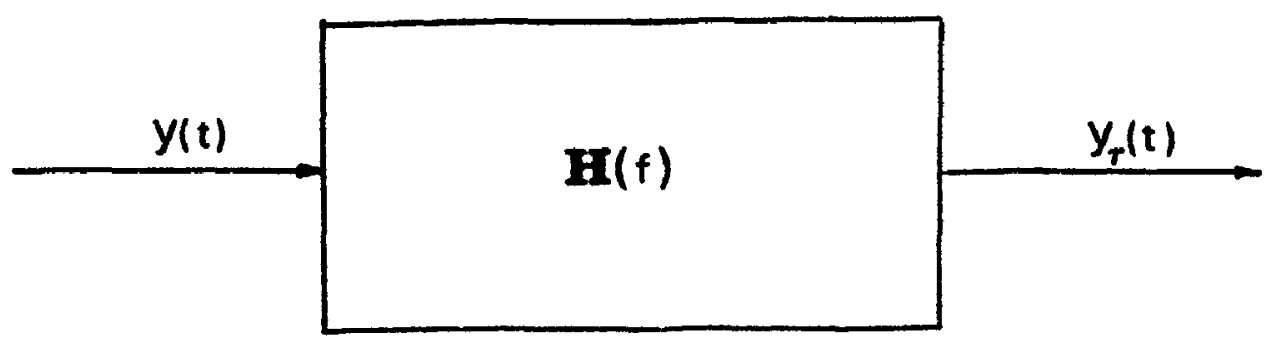

(a)

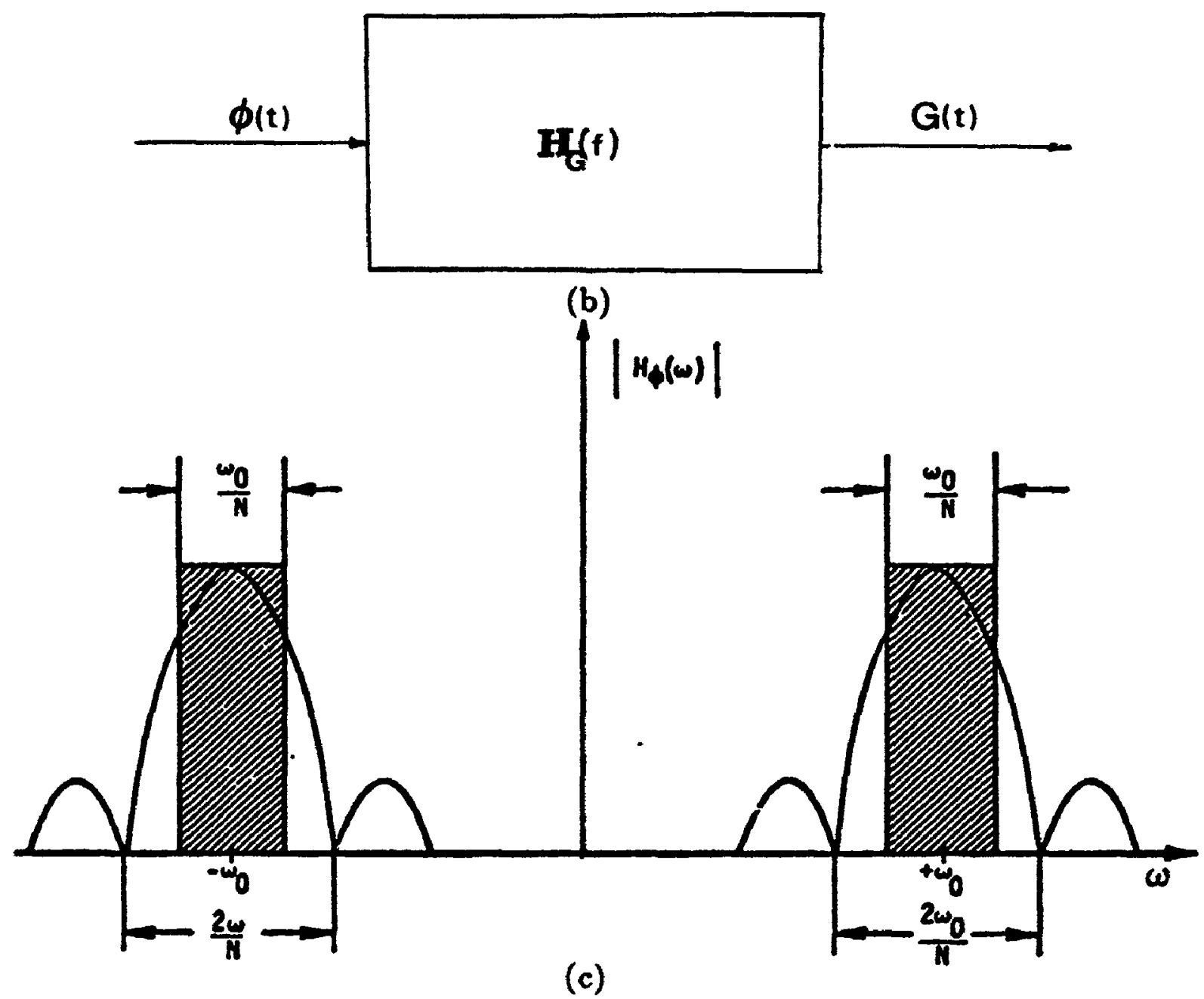

Figure 3.2: (a): The counter as a black box; (b): the counter and the "function generator"; (c): the shaded area represents the equivalent ideal filter 
where $\varphi(t)$ is the instantaneous phase of the oscillator and $S_{\varphi}(f)$ the respective PSD as denoted in the previous chapters. To calculate $H_{G}(f)$ one can proceed as follows:

$$
\begin{aligned}
G(t) & =\frac{\alpha_{0}}{2 \pi \tau}[\varphi(i)-\varphi(t-\tau)]+\frac{\alpha_{1}}{2 \pi \tau}\left[\varphi\left(t-T_{1}\right)-\varphi\left(t-T_{1}-\tau\right)\right] \\
& +\cdots+\frac{\alpha_{M}}{2 \pi \tau}\left[\varphi\left(t-M T_{1}\right)-\varphi\left(t-M T_{1}-\tau\right)\right]
\end{aligned}
$$

Taking Fourier transforms in eq.(3.4) we get:

$$
H_{G}(\omega)=\left(1-e^{j \omega \tau}\right)\left[\frac{\alpha_{0}}{2 \pi \tau}+\frac{\alpha_{1}}{2 \pi \tau} e^{-j \omega T_{1}}+\frac{\alpha_{2}}{2 \pi \tau} \epsilon^{-j 2 \omega T_{1}}+\cdots+\frac{\alpha_{M}}{2 \pi \tau} e^{-j M \omega T_{1}}\right]
$$

or

$$
H_{G}(f)=f\left[\alpha_{0}+\alpha_{1} e^{-j \omega T_{1}}+\alpha_{2} e^{-j 2 \omega T_{1}}+\cdots+\alpha_{M} e^{-j M \omega T_{1}}\right] \frac{\sin \pi f \tau}{\pi f \tau} e^{-j \pi f \tau}
$$

and the magnitude squared is:

$$
\left|H_{G}(f)\right|^{2}=f^{2}|G(f)|^{2}\left(\frac{\sin \pi f \tau}{\pi f \tau}\right)^{2}
$$

with

$$
G(f) \triangleq\left|\alpha_{0}+\alpha_{1} e^{-j 2 \pi f T_{1}}+\cdots+\alpha_{M} e^{-j 2 \pi f M T_{1}}\right|
$$


Now it is easy to see how useful the "Transfer Function" approach can be, because we can approximate a desired filter $G(f)$ by choosing suitable values for the $\alpha_{i}$ factors. As an example [Peregrino 76] consider $\alpha_{i}=(-1)^{i}$ which gives:

$$
G(t)=\bar{y}_{0}-\bar{y}_{1}+\bar{y}_{2}-\bar{y}_{3}+\cdots+\bar{y}_{N-2}-\bar{y}_{N-1}
$$

where $N-1$ is an odd number. Eqns.(3.8) and (3.7) can easily give:

$$
|G(f)|^{2}=\left(\frac{\sin N \pi T_{1} f}{\cos \pi T_{1} f}\right)^{2}
$$

which is exactly the Hadamard Variance (eqn.(2.46)). This filter can be shown to be much narrower than the Barnes or Boileau equivalent filters given in Chapter 2. This means that it is more suitable to measure spectral density if it is needed. The reason for that can be easily seen because the objective is to make the counter equivalent to a very narrow bandpass filter. This will allow us to estimate $S_{\varphi}\left(f_{0}\right)$ at a particular frequency $f_{0}$, for eqn.(3.3) as follows:

$$
S_{\varphi}\left(f_{0}\right) \approx \frac{\hat{\sigma}_{G}^{2}}{\left|H_{G}\left(f_{0}\right)\right|^{2} \frac{2 f}{(N+1)}}
$$

Figure 3.2(c) shows this more clearly. $\omega_{0}$ is equal to $\pi / T_{1}$.

Concluding we may say that this section explains the implementation of the transfer function approach using a frequency counter. Using the counter we can even perform spectral analysis as well as TD stability analysis through a suitable choice of $\alpha_{i}$. This can be done provided that the resulting closed form expressions do satisfy the requirements of the design. This last aspect can be investigated by employing the method developed in Chapter 2. 


\subsection{ADAPTIVE TECHNIQUES USING TIME- DOMAIN MEASURES TO OBTAIN AN ACCEPTABLE ERROR FOR VCO DRIFT CANCELLATION}

The increasing applicability of adaptive digital filter algorithms in subjects such as noise cancellation, spectral estimation frequency estimation and system identification has led researchers to develop single and multiple processor architectures for implementing such filters [Falconer 80], [Barnes 86]. In this way real-time adaptive filtering can be performed with minimum possible speed limitations. On the other hand the applications of microprocessors even to the design of simple analog frequency [Sutoria 82] and temperature [Gerber 85] compensating circuits indicates their potential as processing and controlling elements in the design of more complex systems. Therefore microprocessors have become a necessary building element in modern control and communications systems.

Taking advantage of the above developments and considerations it is important to investigate the applicability of adaptive algorithms to frequency hopping synthesizers and more specifically the ability of such algorithms to correct systematic frequency departures. Such departures of moderate magnitudes can bring a phase-locked synthesizer out of lock whereas the system under study (as will be demonstrated in Chapter 5) can always be kept in frequency lock even for frequency drifts of several MHz per sec.

It is the purpose of this section to investigate the application of adaptive algorithms to the type of frequency hopping being investigated in this thesis, in order to correct for systematic frequency departures due to:

(a) Temperature variations. 
(b) Slow discharging of the battery used to bias the VCO.

(c) Analog and digital noise, coupled to the de supply line as well as to the tuning input voltage line of the VCO.

A brief description of all these sources of frequency disturbance is given below.

(a) Temperature variations are the major source of systematic and monotonic (on the average) frequency departures. But since temperature variations are directly related to an exponential with large time constant, the effect on the output is a slowly changing frequency. The overall frequency departure can be quite large since usually there is a $2-3 \mathrm{MHz}$ frequency change for every $10^{\circ} \mathrm{C}$ of temperature change.

(b) A slowly-varying VCO power-supply voltage has an effect similar to temperature variations, the only difference being that the VCO can immediately respond to an abrupt supply voltage change. However abrupt voltage changes do not usually occur whereas a slowly-varying DC source is a quite frequent phenomenon.

(c) Analog and digital noise coupled to both the voltage supply and the tuning voltage inputs of the VCO can be due to inductive and capacitive coupling between two lines located relatively close to each other [Mohr 67]. This kind of coupling has been shown to produce uncorrelated noise when the transmission lines are long with respect to a wavelength [Cravis 63]. (Example: telephone lines). On the other hand, this type of coupling produces correlated noise when we deal with short coupled line lengths [BTL Tec. Staff 71] (Example: electronic devices and systems). The so-called "victim" lines correspond to the bias and tuning voltage lines of the VCO. Lines producing the coupling can include the standard $400 \mathrm{~Hz}, 1-50 \mathrm{~A}$ aircraft power distribution system lines or the high frequency (up to $400 \mathrm{KHz}$ ) lines used in modern circuitry, or even microprocessor control lines being "contaminated" by a ripple voltage due to poor isolation between input-output stages of the counters. Another source of considerable coupling can be the control lines carrying periodic 
square waveforms [Manassewitsch 76]. VCOs tuned over a bandwidth of $500 \mathrm{MHz}$ or more have modulation sensitivities between 50 and $100 \mathrm{MHz} / \mathrm{V}$. For the best case of $50 \mathrm{MHz} / \mathrm{V}$ we have a $10 \mathrm{KHz}$ frequency deviation for a change of $2 \times 10^{-4}$ volts. Without elaborating it can be shown using suitable equations from [Mohr 67], that if an open wire is used as the $V_{t}$ line, the induced voltage can have an amplitude of 1 to $10 \mathrm{mV}$. If coaxial cable is used for shielding purposes this noise voltage can be reduced to $10^{-4}$ volts which can still affect the VCO output as can be seen from the previous discussion.

\section{(a) Predictive Capability Requirements}

The main objective here is to use adaptive ilgorithm to correct for frequency departures caused by the three previous disturbances. Adaptive algorithms have been extensively used for system identification, spectral estimation, frequency estimation and prediction in a great variety of communication systems during recent years [Falconer 78], [Widrow 80], [Stein 88]. The two basic forms have been developed by R. Kalman [Kalman 61] and by B. Widrow [Widrow 76] and are known as the Kalman and LMS (Least Mean Square) algorithms respectively.

First of all we cannot afford a large number of iterations in order to achieve a reasonable mean square error (MSE) because:

(a) We do not know how often these disturbances will occur.

(b) They will usually happen at random and independently in the various transmitter-receiver modems, which means that not all of them will present the same problem simultaneously (as happens for example, with problems related to channel equalization). On the other hand every unit will be affected if one of them experiences a severe frequency departure. Hence the probability of poor communication between the various modems can be very high. Another thing we have to consider is the rate of frequency change which, in the case of the most severe 
frequency departure, can be 0.1 to $0.30 \mathrm{MHz} / \mathrm{sec}$ at the most. This can happen for the case of a slowly-discharging battery. This corresponds to $20-50 \mathrm{mV} / \mathrm{sec}$ voltage change on the dc bias as has been experimentally determined using two VCOs. For a more severe voltage change, the battery will completely fail within a few minutes. In this case, any prediction is not needed, since the battery will have to be immediately replaced.

Hence, we can consider that the predictive algorithm is satisfactory if the following two requirements are fulfilled:

1. It converges fast (within 30 iterations) within reasonable MSE.

2. It goes to a relatively small MSE on the average, after conversion. (A relatively small MSE average can be considered to be 1.2 to 1.3 times the noise variance).

The following is the correction procedure.

(a) When a considerable frequency departure is detected by the counter, the predictive algcrithm starts working until it has converged to relatively small MSE after a reasonably small number of iterations.

(b) After reaching convergence, the cumulative error is corrected by modifying the DAC word appropriately.

(c) The algorithm is now at steady state and the error is corrected continuously every few iterations, depending on the variance level.

Hence the main idea is that first we wait for the algorithm to converge and then we correct the error for the first time. This type of correction is, of course, based on the assumption that the frequency change is linearly related to the VCO tuning voltage in the neighborhood of a specific tuning voltage $V_{t}$. This is a very 
reasonable assumption since we are talking about linearity of the tuning curve over a frequency range of $15-200 \mathrm{KHz}$ while the bandwidth of the device is around 500 MHz.

(b) LMS Algorithm

A general form of an adaptive tapped-delay-line predictive error structure usually called a filter is shown in Figure 3.3. The input signal $x(t)$ is sampled and we get (the samples) $x(k)$ where $k$ is an integer. Then this is delayed by $d$ samples. Hence we form the signal vector:

$$
X^{T}(k)=[x(k-d), \quad x(k-d-1), \cdots x(k-d-L+1)]
$$

where the exponent $T$ signifies vector transposition. $x(t)$ represents the departure of the VCO output frequency from its nominal value. Now the output of the predictor at the $k$-th instant, based on $L$ previous data points, will be [Widrow 76], [Widrow 85]:

$$
\hat{x}(k)=\sum_{\ell=0}^{L-1} w_{\ell}(k) \cdot x(k-d-\ell)=X^{T}(k) \cdot W(k)
$$

where $W(k)$ is the weight coefficients vector. Hence the error at the $k$-th time instant will be:

$$
\epsilon(k)=x(k)-\hat{x}(k)=x(k)-X^{T}(k) \cdot W(k)
$$

and the mean square error will be:

$$
\xi \triangleq E\left\{\epsilon^{2}(k)\right\}
$$




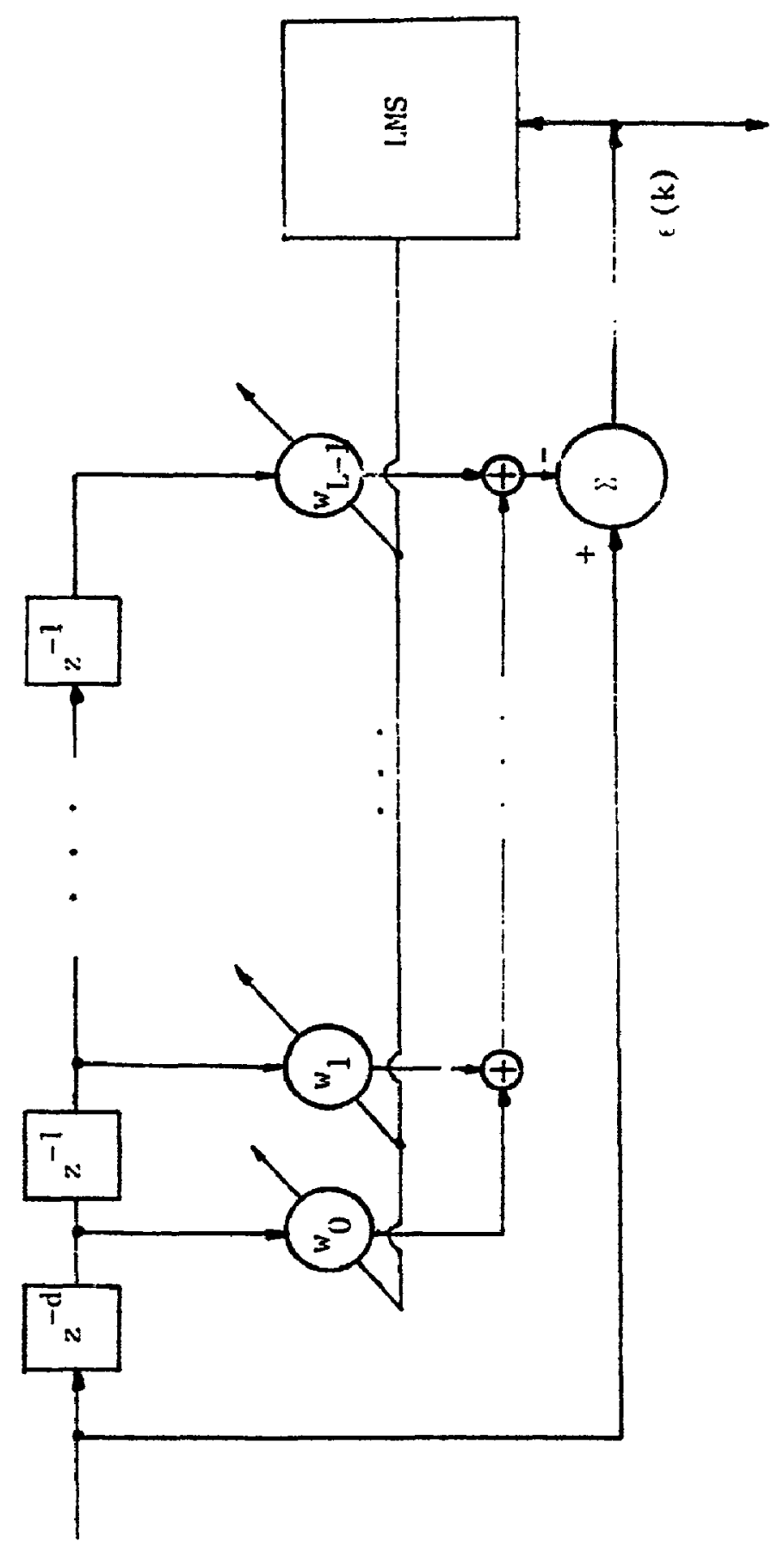

Figure 3.3: General form of adaptive linear prediction filter 
Now we take an estimate of the gradient using the square of a single error sample $\epsilon^{2}(k)$ [Widrow 85]:

$$
\hat{\nabla}_{k}=\left[\frac{\partial \epsilon^{2}(k)}{\partial w(0)}, \cdots \frac{\partial \epsilon^{2}(k)}{\partial w(L)}\right]^{T}=2 \epsilon(k) \cdot\left[\frac{\partial \epsilon(k)}{\partial w(0)}, \cdots \frac{\partial \epsilon(k)}{\partial w(L)}\right]^{T}
$$

which is equal to:

$$
\hat{\nabla}_{k}=-2 \epsilon(k) \cdot X(k)
$$

Hence the LMS algorithm can be written as follows:

$$
W(k+1)=W(k)-\mu \hat{\nabla}_{k}
$$

or

$$
W(k+1)=W(k)+2 \mu \cdot \epsilon(k) X(k)
$$

or in terms of individual vector components:

$$
\left.\begin{array}{c}
w_{\ell}(k+1)=w_{\ell}(k)+2 \mu \cdot \epsilon(k) x(k-d-\ell) \\
\ell=0,1, \cdots, L-1
\end{array}\right\}
$$

To derive the algorithm, Widrow [Widrow 76] assumes that the input signals and desired outputs (estimates) are stationary ergodic processes and that the input vector $X(k)$ is uncorrelated over time $\left(E\left\{X(k) \cdot X^{T}(k+i)\right\}=0 \forall i \neq 0\right)$. Note that stationarity of the input vector and decorrelation are not necessary for convergence of the LMS algorithm ([Widrow 85], p.103, [Bitmead 80]). 


\subsection{COMPARISON OF MCMH WITH THE OTHER SYNTHESIS TECHINIQUES IN THE CONTEXT OF FREQUENCY HOPPING}

In the previous sections a typical system representing an application of the TD stabilixy characterization has been laid out. Its main requirements and subsystems have been discussed in detail and adaptive techniques for acquiring frequency locking within $\mathbf{0 . 0 0 1 \%}$ of the final value have been outlined. Before proceeding to demonstrate the ability of adaptive algorithms to perform this type of frequency correction (Chapter 4) and finally to implement fully such a system and measure its performance and stability characteristics (Chapter 5 ), it is beneficial to demonstrate its special capabilities due to the particular implementation approach adopted here.

(a) Design Criteria and Flexibility of Implementation

Most of the features of this system can be changed as desired except for the tuning speed because the setting time appears to have a low limit of $1 \mu \sec$ when only one VCO is used. By changing the VCO we can change the output frequency range. By increasing the number of VCOs we can even speed it up. This can be done using a switch to allow the system to process and adjust the output of one VCO while the other is hopping. By simply changing some instructions and parameters in the controlling program in the $\mu \mathrm{P}$ we can have different numbers of frequencies and different frequency steps. Different frequency patterns can also be employed which can be pseudorandom, linear sweeping, etc. By adjusting the counting interval $\tau$ and the output bandwidth we can alter the frequency resolution.

(b) Comparison With Other Techniques

The absence of spurious responses is very important especially for Electronics 
Support Measures (ESM) systems [Drapac 87]. Since the operating frequency range is wide the problem is significant in an environment where we have many emitters present within a narrow bandwidth. In this design there is only one mixing stage to convert the output of the VCO into a countable IF. However hybrid multiloop systems have always more than 4 mixers (see Figure 1.6(a)). This increases the number of spurious frequencies. Even DDS systems exhibit discrete noise spikes which have to be removed [Drapac 87]. Hence in terms of amount of spurious, the system at hand seems to have an advantage compared to hybrid systems.

Compared with hybrid systems the present system exhibits almost infinite frequency locking and acquisition ranges as most of the typical FLL systems have. Its speed is also at least an order of magnitude higher than these systems. On the other hand DDS systems are faster but they have a limited output frequency range. Therefore DDS systems are good candidates for the fine step portion of a hybrid multiloop synthesizer but they cannot stand alone if operation with bandwidths and output frequencies of the order of hundreds of $\mathrm{MHz}$ is needed. 


\section{Chapter 4}

\section{FREQUENCY CORRECTION USING ADAPTIVE FILTERING TECHNIQUES}

\subsection{INTRODUCTION}

In Chapter 3 some of the main adaptation requirements of the system were outlined. Since one of the main concerns of adaptive correction of systematic frequency drifts in our system is whether it is able to converge fast and without using elaborate processing and excessive amount of calculations, it is of interest to investigate ways of doing that. The Kalman algorithm [Kalman 61] converges faster than the LMS algorithm but it involves a lot of calculations because the inverse of an autocorrelation matrix is needed at each iteration. On the other hand, the LMS algorithm might be slower than expected for system correction.

Therefore the main purpose of this chapter is to outline a modified version of the LMS algorithm and its ability to converge faster than the simple LMS. This is mainly due to the specific nature of the measured frequency data (MFD) of the system as will be seen shortly in the following sections. 
In the next section the modified LMS algorithm is developed and its stability and speed of adaptation are considered. In Section 3 some results are presented comparing the performances of simple LMS to the modified version by using actual measured frequency data from an early version of the system.

\subsection{THE MODIFIED LMS ALGORITHM (MDLMS)}

\subsubsection{Mathematical Formulation}

In Chapter 3 we outlined the simple LMS algorithm which is given by the following equations:

$$
\left.\begin{array}{c}
\hat{x}(k)=\sum_{\ell=0}^{L-1} w_{\ell}(k) \cdot x(k-d-\ell) \\
w_{\ell}(k+1)=w_{\ell}(k)+2 \mu \cdot \epsilon(k) \cdot x(k-d-\ell) \\
\ell=0,1, \cdots L-1
\end{array}\right\}
$$

where $\epsilon(k)$ is the error at time instant $k, \mu$ is the adaptation constant and $w_{\ell}(k)$ the individual weight vector components.

Before proceeding to the modified LMS algorithm (MDLMS) it is of importance to mention that:

(a) A unit distance prediction is used here $(d=1)$.

(b) All vectors are of dimension $3(L=3)$.

Actually $L$ can be even increased up to 4 . For higher values of $L$, the amount of calculations to be carried out at each iteration cannot be performed within the 
dwell time limit.

Disturbances such as long-term frequency drifts, abrupt temperature changes or slowly-varying VCO power-supply voltages can be represented by using the following model for the counted frequency:

$$
x(t)=\omega_{0, s}+\left(\omega_{1, t} \cdot t\right)+v(t)
$$

Here again, $x(t)$ represents the departure of the VCO output frequency from its nominal value. When none of the previous disturbances is present, the term $v(t)$ is referred to as the short term frequency instability whose behaviour was examined in Chapter 2.

In terms of discrete samples:

$$
x(i T)=\omega_{0, i}+\left(\omega_{1, i} \cdot i T\right)+v(i T)
$$

where $w_{0, i}$ and $w_{1, i}$ represent respectively the constant frequency term and the slope of the data versus time at a specific instant iT. The signal can also be written in the following form:

$$
x(i T)=\hat{\omega}_{0, i}+\left(\hat{\omega}_{1, i} \cdot i T\right)+v(i T)
$$

where $\hat{w}_{0, i}$ and $\hat{w}_{1, i}$ are reasonably good estimates of $w_{0, i}$ and $w_{1, i}$ respectively. $\hat{w}_{0, i}$ and $\hat{w}_{1, i}$ are $\varepsilon=t u a l l y$ random variables. Maximum Likelihood Estimates of $w_{0, i}$ and $w_{1, i}$ are derived in Appendix 4.1. As indicated above, $T$ is an integer representing the time interval between two successive measurements, in secs. By replacing $[\hat{R}(k)]^{-1}$ with $[E\{\hat{R}(k)\}]^{-1}$, the updating equations for the weight vector in Kalman-SER algorithms [Widrow 85] becomes: 


$$
W(k+1)=W(k)+\frac{2(\ell m)\left(1-\lambda^{k+1}\right)}{1-\lambda}[E\{\hat{R}(k)\}]^{-1} \cdot \epsilon(k) \cdot X(k)
$$

Where $\mathrm{lm}$ is a constant directly proportional to the average of the eigenvalues $\lambda_{a v}$ of $R(0<\ell m<1) . \hat{R}(k)$ is the estimate of the autocorrelation matrix given by

$$
\left.\begin{array}{c}
\hat{R}(k)=\frac{1-\lambda}{1-\lambda^{k+1}} \sum_{t=0}^{k} \lambda^{k-2} X_{0} X_{a}^{T} \\
0<\lambda<1 \quad, \lambda=2^{-1 / L S X}
\end{array}\right\}
$$

where LSX is the length of stationarity of $X$ (i.e., the number of samples during which $X$ is stationary), the exponent $T$ signifies vector transposition. Using $[E\{\hat{R}(k)\}]^{-1}$ instead of $[\hat{R}(k)]^{-1}$ is a matter of omitting second order statistics as is explained in Appendix 4.2. We want to calculate $E\{\hat{R}(k)\}$ first:

$$
E\{\hat{R}(k)\}=\sum_{s=0}^{k} \lambda^{k-s} \cdot E\left\{X_{*} X_{s}\right\}
$$

where

$$
\left.\begin{array}{l}
X_{s} \triangleq\left[x_{1 s} x_{2 s} x_{3 s}\right]^{T} \\
x_{1 s} \triangleq x[(s-1) T] \\
x_{2 s} \triangleq x[(s-2) T] \\
x_{3 s} \triangleq x[(s-3) T]
\end{array}\right\}
$$

Now using (4.4), (4.6a), (4.6b) and (4.7) we can derive expressions for $E\{\hat{R}(k)\}$. Only the main equations are given here. A detailed derivation is given in Appendix 4.3. For general $k, j$ such that $|k-j|=0,1,2$ we have: 


$$
\begin{aligned}
E\{x(k T) \cdot x(j T)\} & =E\left\{x_{k} \cdot x_{j}\right\}=E\left\{\hat{\omega}_{0, k-1} \cdot \hat{\omega}_{0, j-1}\right\} \\
& +T(k-1) \cdot E\left\{\hat{\omega}_{0, j-1} \cdot \hat{\omega}_{1, k-1}\right\}+T \cdot(j-1) \cdot E\left\{\hat{\omega}_{0, k-1} \cdot \hat{\omega}_{1, j-1}\right\} \\
+ & T^{2} \cdot(k-1) \cdot(j-1) \cdot E\left\{\hat{\omega}_{1, k-1} \cdot \hat{\omega}_{1, j-1}\right\}+\sigma_{x, k-j}^{2} \\
& \sigma_{x, k-j}^{2} \triangleq E\{v(k-1) \cdot v(j-1)\}
\end{aligned}
$$

As mentioned above, $\sigma_{x, k-j}^{2}$ represents the cumulative effect of short-term disturbances on the frequency source. This noise is assurned to be white Gaussian noise which means that $\sigma_{x, k-j}^{2}=0$ for $k \neq j$. This assumption is valid when the equations to be derived here are to be used for prediction of a region of data which represents a straight line plus white noise. One can usually subdivide the measured frequency data (MFD) versus time curves into regions which present this property, since long term drifts such as effects due to temperature variations and slowly-varying supply voltages correspond to slow monotonic (on the average) frequency departures. Figure 4.1 represents a typical MFD versus time curve.

Later we will see that by retaining only a predominant term of the estimated inverse autocorrelation matrix, the algorithm is transformed into an LMS with time-varying $\mu$ and the previous assumption is no longer needed.

Proceeding further in calculating the terms of (4.6a) we get: 


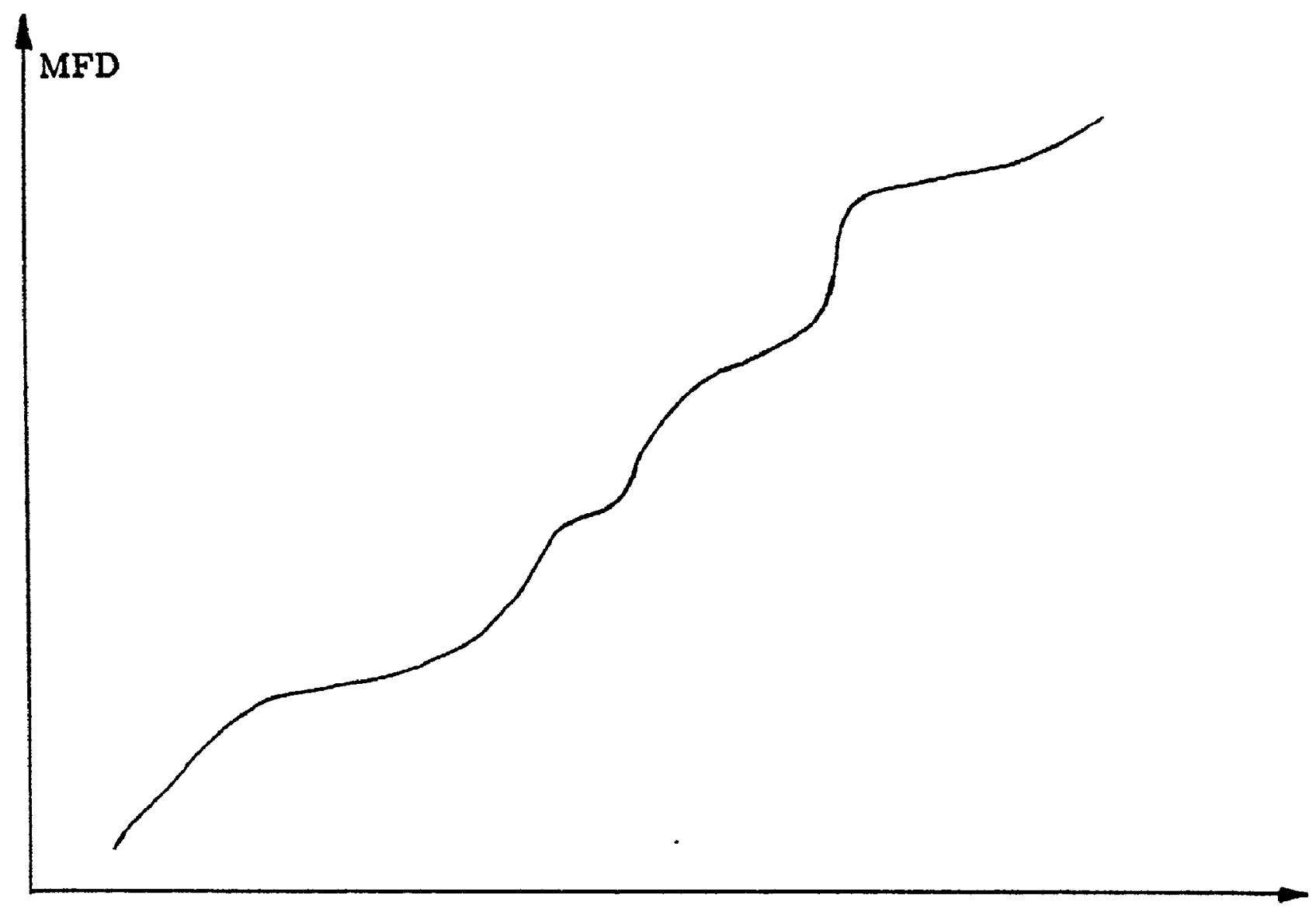

Time

Figure 4.1: Typical measured frequency data (MFD) versus time curve 


$$
\begin{aligned}
& E\left\{\hat{r}_{11}(n)\right\} \triangleq q_{11}(n)=\sigma_{0}^{2}+F_{1}-2\left(C_{1,1}+C_{2,1}\right)+C_{3,1} \\
& q_{12}(n)=F_{2}-3\left(C_{1,2}+C_{2,2}\right)+2 C_{3,2} \\
& q_{13}(n)=F_{3}-4\left(C_{1,3}+C_{2,3}\right)+3 C_{3,3} \\
& q_{22}(n)=\sigma_{0}^{2}+F_{2}-4\left(C_{1,2}+C_{2,2}\right)+4 C_{3,2} \\
& q_{23}(n)=F_{3}-5\left(C_{1,3}+C_{2,3}\right)+6 C_{3,3} \\
& q_{33}(n)=\sigma_{0}^{2}+F_{3}-6\left(C_{1,3}+C_{2,3}\right)+9 C_{3,3}
\end{aligned}
$$

Expressions for all right-hand-side terms of equations (4.10) are given in Appendix 4.3 where it is also explained that for $n \geq 10$ these expressions can be replaced by the following, resulting in an error of less than 3-5\%.

$$
\left.\begin{array}{l}
q_{11}(n)=\sigma_{0}^{2}+F-2\left(C_{1}+C_{2}\right)+C_{3} \\
q_{12}(n)=F-3\left(C_{1}+C_{2}\right)+2 C_{3} \\
q_{13}(n)=F-4\left(C_{1}+C_{2}^{\prime}\right)+3 C_{3} \\
q_{22}(n)=\sigma_{0}^{2}+F-4\left(C_{1}+C_{2}\right)+4 C_{3} \\
q_{23}(n)=F-5\left(C_{1}+C_{2}\right)+6 C_{3} \\
q_{33}(n)=\sigma_{0}^{2}+F-6\left(C_{1}+C_{2}\right)+9 C_{3}
\end{array}\right\}
$$

where

$$
\begin{gathered}
\sigma_{0}^{2}(n) \triangleq \sum_{s=0}^{n} \lambda^{n-s} \sigma_{x, 0}^{2} \\
F(n) \triangleq \sum_{s=0}^{n} \lambda^{n-s} \overline{\hat{\omega}_{0, s-1}^{2}}+2 T \sum_{s=0}^{n} \lambda^{n-s} \cdot s \cdot \overline{\hat{\omega}_{0, s-1} \cdot \hat{\omega}_{1, s-1}}+
\end{gathered}
$$




$$
\begin{aligned}
& +T^{2} \sum_{s=0}^{n} \lambda^{n-\bullet} \cdot s^{2} \overline{\hat{\omega}_{1, n-1}^{2}} \\
& C_{1}(n) \triangleq T^{\prime} \sum_{s=0}^{n} \lambda^{n-s} \overline{\hat{\omega}_{0, s-1} \cdot \bar{\omega}_{1, s-1}} \\
& C_{2}(n) \triangleq T^{2} \sum_{s=0}^{n} \lambda^{n-s} \cdot s \cdot \overline{\hat{\omega}_{1, s-1}^{2}} \\
& C_{3}(n) \triangleq T^{2} \sum_{s=0}^{n} \lambda^{n-s} \overline{\hat{\omega}_{1, s-1}^{2}}
\end{aligned}
$$

Here all horizontal bars represent $E\{\}$. It is interesting to see the form of these equations for a straight-line section of the frequency data. In this case all $\dot{\omega}_{0}$ are identical, and all $\hat{\omega}_{1}$ are equal to each other as well:

$$
\begin{aligned}
& \sigma_{0}^{2}(n) \triangleq \sum_{0=0}^{n} \lambda^{n-8} \sigma_{x, 0}^{2} \\
& F(n) \triangleq S_{1}(n) A_{00}+2 S_{2}\left(r_{i}\right) A_{0} A_{1} T+S_{3}(n) A_{11} T^{2} \\
& C_{1}(n) \triangleq S_{1}(n) A_{0} A_{1} T \\
& C_{2}(n) \triangleq S_{2}(n) \cdot A_{11} T^{2} \\
& C_{3}(n) \triangleq S_{1}(n) \cdot A_{11} \cdot T^{2}
\end{aligned}
$$

where 


$$
\begin{aligned}
& A_{0} \triangleq E\left\{\hat{\omega}_{0}\right\}, A_{1} \triangleq E\left\{\hat{\omega}_{1}\right\} \\
& A_{00} \triangleq E\left\{\hat{\omega}_{0}^{2}\right\}, A_{11} \triangleq E\left\{\hat{\omega}_{1}^{2}\right\} \\
& S_{1}(n) \triangleq \sum_{s=0}^{n} \lambda^{n-s} \\
& S_{2}(n) \triangleq \sum_{s=0}^{n} s \lambda^{n-s} \\
& S_{3}(n) \triangleq \sum_{s=0}^{n} s^{2} \lambda^{n-s}
\end{aligned}
$$

Closed-form and recursive expressions for $S_{1}(n), S_{2}(n), S_{3}(n)$ are given in Appendix 4.4.

The reasons for adopting the approximations reducing equations (4.10), to (4.11), are stated below:

(a) We get simpler expressions for $q_{i j}, i, j=1,2,3$.

(b) The coefficients of the part with the larger magnitude $F$ are all 1.

In this way, when we extract formulas for $[E\{\hat{R}(n)\}]^{-1}$, many simplifications occur. On the other hand, although equations (4.11) are accurate expressions for $q_{i j} s$ for $n \geq 10$, they are different from the exact values for $n \leq 10$. However they still retain the correct qualitative behaviour as terms of the autocorrelation matrix. This will be seen in detail as we proceed further. An obvious consequence of this approximation is that $\hat{R}(n)$ will not be a good approximation to $R(n)$ after a few iterations as happens with the Kalman-SER algorithms but will require around 10 iterations to do that. This can also be seen from the results of the last part.

The elements of the inverse matrix $[E\{\hat{R}(k)\}]^{-1}$ are:

$$
\alpha_{i j}=\frac{(-1)^{i+j} D_{i j}}{D_{0}} \quad i, j=1,2,3
$$


where $D_{i j}$ are the minors and $D_{0}$ is the determinant of the $3 \times 3$ symmetric matrix with $q_{i j}$ as its elements. Again in Appendix 4.3 we derive the following simplified expressions for $D_{i j}, D_{0}$ :

$$
\begin{aligned}
& D_{11} \simeq \sigma_{0}^{2}\left\{\sigma_{0}^{2}+2 F-10\left(C_{1}+C_{2}\right)\right\} \\
& D_{12} \simeq \sigma_{0}^{2}\left\{F-3\left(C_{1}+C_{2}\right)\right\} \\
& D_{13} \simeq \sigma_{0}^{2}\left\{-F+4\left(C_{1}+C_{2}\right)\right\} \\
& D_{22} \simeq \sigma_{0}^{2}\left\{\sigma_{0}^{2}+2 F-8\left(C_{1}+C_{2}\right)\right\} \\
& D_{23} \simeq \sigma_{0}^{2}\left\{F-5\left(C_{1}+C_{2}\right)\right\} \\
& D_{33} \simeq \sigma_{0}^{2}\left\{\sigma_{0}^{2}+2 F-6\left(C_{1}+C_{2}\right)\right\} \\
& D_{0} \simeq\left(\sigma_{0}^{2}\right)^{2}\left\{\sigma_{0}^{2}+3 F\right\}
\end{aligned}
$$

Equations (4.5), (4.14) and (4.15) constitute the modified MDLMS algorithm. It can be seen from these equations that another way to derive MDLMS is to use the orthogonalized LMS algorithm [Widrow 84]:

$$
W_{k+1}=W_{k}+2 \mu \cdot+\epsilon_{k}\left\{E\left[X_{k} \cdot X_{k}^{T}\right]\right\}^{-1} \cdot X_{k}
$$

and select

$$
\left.\begin{array}{l}
\omega_{0, n}^{2} \triangleq \sum_{k=0}^{n-1} \lambda^{n-k-1} \cdot \hat{\omega}_{0, k}^{2} \\
\omega_{0, n} \cdot \omega_{1, n} \triangleq \sum_{k=0}^{n-1} \lambda^{n-k-1} \cdot \hat{\omega}_{0, k} \cdot \hat{\omega}_{1, k}
\end{array}\right\}
$$

There will be some slight modifications of (4.12) but they will retain the same structure. Hence their values as a function of $n$ will be close to the existing expressions. We now set: 


$$
\sigma_{0, I N T}^{2}(n)=A \cdot \sigma_{0}^{2} \cdot \lambda^{n}+\sigma_{0}^{2}
$$

where $A$ is a large constant. We do this in order to establish stability in the first few iterations. This becomes more obvious if we write the equation for the updating of the first element of the weight vector from (4.5):

$$
\left.\begin{array}{ll} 
& w_{0}(k+1)=w_{0}(k)+C_{A}(k) \cdot E_{1}(k) \\
\text { where } & C_{A}=\frac{2(l m)\left(1-\lambda^{k+1}\right)}{1-\lambda} \cdot \epsilon(k) \\
& E_{1}(k)=\alpha_{11} x_{k}(1)+\alpha_{12} x_{k}(2)+\alpha_{13} x_{k}(3) \cdot
\end{array}\right\}
$$

Using (4.14) and (4.15) we get for a particular iteration:

$$
E_{1}=\frac{F[2 x(1)-x(2)-x(3)]+\sigma_{0}^{2} x(1)-\left(C_{1}+C_{2}\right)[10 x(1)-3 x(2)-4 x(3)]}{\sigma_{0}^{2}\left[\sigma_{0}^{2}+3 F\right]}
$$

From equation (4.18) it can be seen that using $\sigma_{0, I N T}^{2}$ in place of $\sigma_{0}^{2}$ is equivalent to using a large initial value $\delta$ in $Q_{0}^{-1}=\delta I$ for the first iteration of the KalmanSER algorithm [Widrow 85]. Apparently $\sigma_{0, I N T}^{2}$ becomes approximately equal to $\sigma_{0}^{2}$ within a few iterations depending on the values of $A$ and $\lambda$. Another way to see it is the following: From (4.12) or (4.13) one can notice that if we choose $T$ such that:

$$
\omega_{1} T \ll 1
$$

then the following relationship is true as well:

$$
\sigma_{0}^{2} \widehat{x(1)} \gg F[\widehat{2 x(1)-x(2)-x(3)}]-\left(C_{1}+C_{2}\right)[\widehat{10 x(1)-3 x(2)-4 x(3)}]
$$


where " stands for statistical average. Of course equation (4.18b) does not hold for any range of values for $x(1)$ but, as has already been emphasized, after some iterations there will be always a correction of the synthesizer's departure from the nominal frequency, which equivalently means that the data sequence $x_{i} i=1 \cdots \infty$ will always be bounded by an upper limit of relatively small magnitude. Note that equation (4.18a) expresses a quantitative condition suggesting equivalently that the lower $\omega_{1} T$ is, the more valid is the replacement of $[\hat{R}]^{-1}$ by $[E\{\hat{R}\}]^{-1}$ in the development of the algorithm as has been done in Appendix 4.2.

Now observing equation (4.18) and taking into account (4.18b), one can see that the predominant term of the denominator is $3 F$ which is directly related, according to equation (4.12b), to the estimated average signal power in the neighbourhood of $n$. Hence a highly simplified form of the algorithm can be thought of as a simple LMS with time-varying $\mu$ as follows:

$$
\left.\begin{array}{c}
w_{\ell}(k+1)=w_{\ell}(k)+2 \mu_{e q}(k) \cdot \epsilon(k) \cdot x(k-\ell-1) \\
\ell=0,1,2
\end{array}\right\}
$$

Let us call this "the modified LMS" or MLMS algorithm, it is this form that will be investigated in more detail in the following sections because of its evident usefulness due to its simplicity. This MLMS algorithm could also have been derived by using the known result for LMS [Haykin 84]:

$$
\mu(k)=\frac{1}{\operatorname{trace}(\mathrm{R})}=\frac{1}{\sum_{i=1}^{3} E\left[x_{k}^{2}(i)\right]}
$$

where again equations ( $4.15 \mathrm{~b})$ have to be used. 
Some justification for the effectiveness of equation (4.19b) will now be given. We do this by comparing it with the LMS algorithm with time varying $\mu_{\text {eq }}$, which has been discussed by various authors (e.g., [Widrow 85]). According to this approach:

$$
\mu_{e q}(k)=\frac{1}{P_{a v}(k)}
$$

We know also (Appendix 4.3) that:

$$
F(k)=\lambda F(k-1)+2 k T \overline{\hat{\omega}_{0, k} \cdot \hat{\omega}_{1, k}}+\overline{\hat{\omega}_{0, k}^{2}}+T^{2} k^{2} \overline{\hat{\omega}_{1, k}}
$$

Now the development of the MLMS, as was done even under restrictive assumptions (Appendix 4.3, equation (4.64)), makes it legitimate to use, not $\boldsymbol{P}_{a v}$ (at k) but $\boldsymbol{P}_{\text {av }}$ (in the neighborhood of $k$ ) in the calculation of $\mu_{\text {eq }}$, where the neighborhood is defined as the region around $k$ where the restrictive assumptions are valid. The width of this region depends on the value of $\lambda$ as well. Now using equation (4.20) we can relax the restrictive assumptions. This leads to the following equations:

$$
\begin{aligned}
F_{\text {accurate }}(k+p) & =\lambda^{p+1} \cdot F(k-1)+\sum_{z=0}^{p} \lambda^{p-z} \overline{\hat{\omega}_{0, z+k}^{2}}+ \\
& +2 T \sum_{z=0}^{p} \lambda^{p-z}(k+z) \overline{\hat{\omega}_{0, z+k} \cdot \hat{\omega}_{1, z+k}}+ \\
& +T^{2} \sum_{z=0}^{p} \lambda^{p-z}(k+z)^{2} \overline{\hat{\omega}_{1, z+k}^{2}}
\end{aligned}
$$




$$
\begin{aligned}
& +2 T \sum_{z=0}^{p} \lambda^{p-z}(k+z) \overline{\hat{\omega}_{0, k} \cdot \hat{\omega}_{1, k}} \\
& +T^{2} \sum_{x=0}^{p} \lambda^{p-z}(k+z)^{2} \overline{\hat{\omega}_{1, k}^{2}}
\end{aligned}
$$

Equation (4.22) implies that we do not have to estimate $\overline{\bar{\omega}_{0}}, \overline{\bar{\omega}_{1}}$ at every iteration between $k$ and $k+p$ but that we can use $\overline{\hat{\omega}_{0, k}}, \overline{\hat{\omega}_{1, k}}$ for the next $p$ iterations. Notice that the probability that the algorithm will diverge increases when the sensitivity $S E$ becomes large:

$$
S E=\frac{F_{\text {epprox }}(k+p)-F_{\text {ccour }}(k+p)}{F_{\text {ccaur }}(k+p)} \triangleq \frac{\Delta F(k+p)}{F(k+p)}
$$

However, $S E$ is quite small (close to zero) during the first $4-5$ iterations ( $p=1$ to 5) because the restrictive assumptions are true 4-5 iterations after estimates for $\omega_{0, k}$ and $\omega_{1, k}$ have been taken. After the first iteration $S E$ is still kept small because although $\Delta F(k+p)$ increases, $F(k+p)$ increases as well because of the nature of the data. Hence we can choose $p$ and $T$ such that we keep SE reasonably small, without having to update $\omega_{0}$ and $\omega_{1}$ at every iteration but only after every $p$ iterations. This method (depending on the value of $p$ ) can reduce the total number of arithmetic operations per $p$ iterations by $30-60 \%$.

From the above presentation and analysis in the appendices of this chapter, one can see that the LMS (equation (4.1)), MDLMS (equations (4.5), (4.14) and (4.15)) and MLMS (equation (4.19)) algorithms are the three obvious alternatives for frequency correction. We examine below the convergence stability and adaptation speed of these three algorithms. 


\subsubsection{Convergence Stability}

It is obvious that MDLMS converges always to the optimum $W^{*}$ since after 8-10 iterations, equations (4.14) and (4.15) approximate $[E\{\hat{R}(k)\}]^{-1}$ quite well. It can also be seen that despite the fact that our "signa" is always an increasing or decreasing (on the average) function of time, the LMS and MLMS algorithms do converge.

For this purpose, Bitmead's "second theorem", [Bitmead 80], is given as follows: "With nonstationary $\phi$-mixing input $\left\{u_{k}\right\}$ with mixing constants $\left\{\phi_{i}\right\}$ satisfying $\sum_{i=1}^{\infty} \phi_{i}^{1 / 2}<\infty$ having $\lim _{n \rightarrow \infty}\left\{\right.$ inf $\left.\left(\frac{1}{n}\right) \sum_{i=1}^{n} E\left\{u_{i} u_{i}^{\prime}\right\}\right\}$ positive definite, then the LMS algorithm is almost surely and mean-square exporentially convergent, provided there exisis a uniform bound $M$ such that $\left|u_{k}\right|=M<\infty$ for all $k$ and $\mu \in\left(0,2 / M^{2}\right)^{n}$. The $\phi$-mixing property implies that the distant future of the process is somehow dependent upon its present and past [Iosifesku 69].

This theorem simply states that under some very loose restrictions the LMS algorithm is exponentially convergent if the data sequence is bounded by a number $M$ and if we use $0 \leq \mu \leq 2 / M^{2}$. Now if we assume that we are trying to predict a data sequence using simple LMS within a specified time interval $\left(0, t_{T O T}\right)$, then choosing $\mu \leq \frac{2}{M^{2}\left(0, t_{T O T}\right)}$ the estimator will converge exponentially according to the above theorem. Using MLMS is equivalent to subdividing the whole data sequence into many separate regions as in Figure 4.2. Then within each region, the prediction is equivalent to a simple LMS. But since in each different region the bound $M$ is different, then $\mu$ is different as well. Hence we have: 


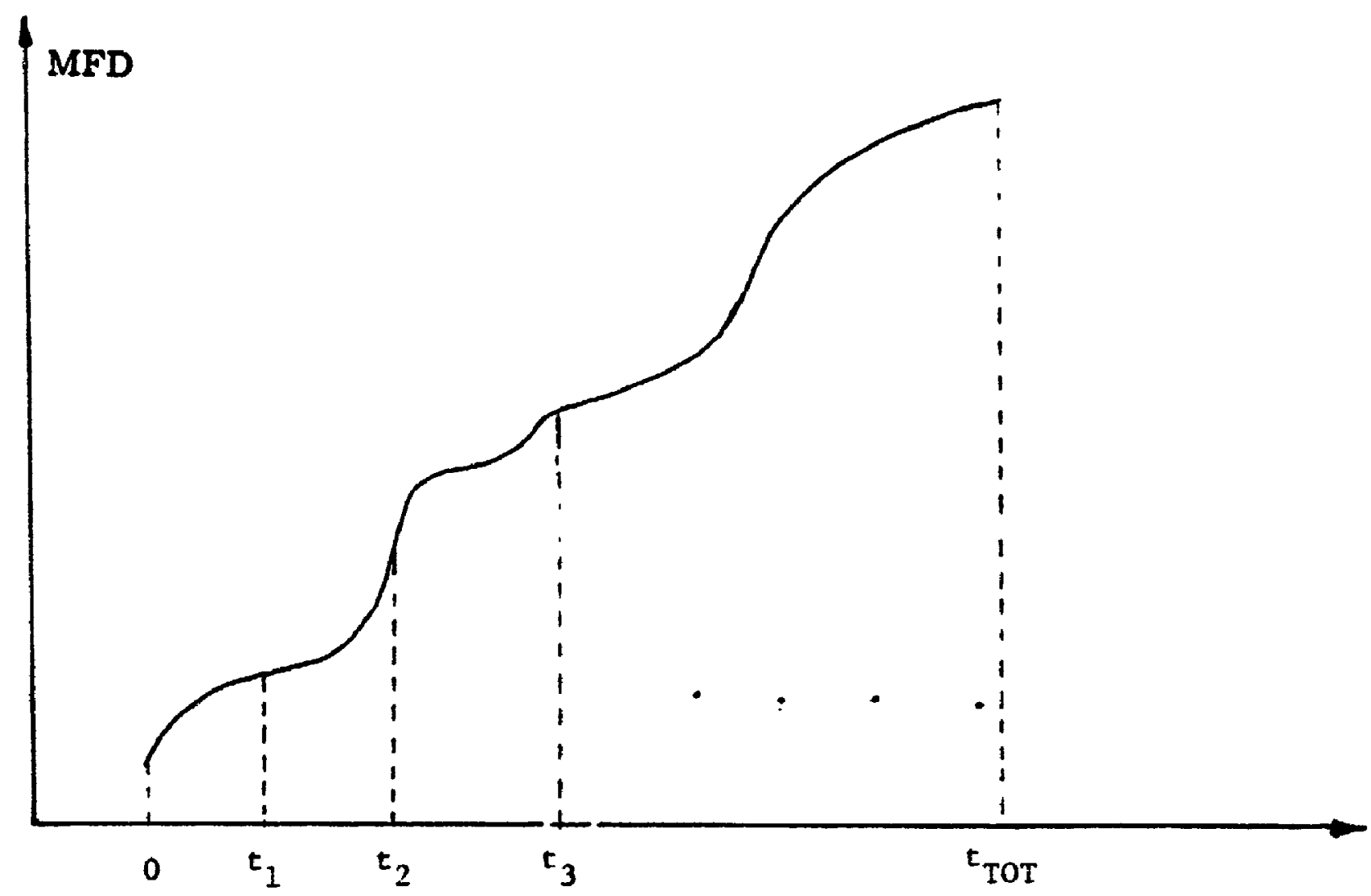

Figure 4.2: Subdivision of MFD into separate regions 


$$
\left.\begin{array}{ccc}
\mu\left(0, t_{1}\right) & \leq & \frac{2}{M^{2}\left(0, t_{1}\right)} \\
\mu\left(t_{2}, t_{2}\right) & \leq & \frac{2}{M^{2}\left(t_{1}, t_{2}\right)} \\
\vdots & & \\
\mu\left(t_{\text {TOT }-1}, t_{\text {TOT }}\right) & \leq & \frac{2}{M^{2}\left(t_{\text {TOT } \left.-1, t_{\text {TOT }}\right)}\right.}
\end{array}\right\}
$$

Equation (4.24) obviously gives:

$$
\mu\left(0, t_{1}\right)>\mu\left(t_{1}, t_{2}\right)>\mu\left(t_{2}, t_{3}\right) \cdots>\mu\left(t_{\text {TOT }-1}, t_{\text {TOT }}\right)=\mu_{L M S}
$$

The RHS's of equation (4.24) are directly proportional to the local "signal power" at the particular time intervals. $F$ is directly related to the estimated (not calculated at each iteration) average local signal power. Hence one can see that the $\mu_{e q}(k)$ in (4.19b) are directly related to the $\mu\left(t_{k-1}, t_{k}\right)$ of equation (4.24) which means that the MLMS algorithm is exponentially convergent as well.

\subsubsection{Speed of Adaptation}

It is well known ([Widrow 76], [Widrow 85], [Haykin 84]) that in the class of adaptive algorithms examined here, the speed of adaptation is mathematically related to an exponential decay of the learning curve, the time constant being inversely proportional to $\mu$. This means that as $\mu$ becomes smaller, the speed of adaptation decreases. It is now obvious after the preceding discussion that MLMS will always be faster than LMS because of equation (4.25) which implies that since $\mu_{\text {MLMS }}(t)$ is initially always larger than $\mu_{\text {LMS }}=$ const, the MLMS algorithm will converge faster than LMS. On the other hand one cannot easily conclude that MDLMS is superior to MLMS in terms of speed. We know that MDLMS will converge within 8 to 10 iterations as mentioned in Section 4.2.2. But depending on the nature of 
the data, MLMS may also converge within the same number of iterations. From the performance of the algorithms with real data as presented in the next part of this chapter it is concluded that MDLMS converges to steady-state 3-6 iterations before MLMS does. This is considered to be a negligible difference and for that reason MLMS is preferred to MDLMS.

In order to speed up the LMS convergence we can use the simple LMS after we have subtracted $\left(\hat{\omega}_{0}+\hat{\omega}_{1} n T\right)$ at each aimost-straight line section of the frequency data. This method is not a different algorithm but a different "processing procedure" and we designate it SLMS. Although it has the same disadvantages as straightforward LMS it is faster than that algorithm. The reason for this is that the new frequency data produced by the subtraction process will have a much smaller average slope with respect to time than do the original data. This new average slope will be directly proportional to the difference between $\omega_{1}$ and $\hat{\omega}_{1}$ in each particular data region. The new shape of the data (SMFD) will have the form shown in Figure 4.3. Hence the equivalent $\mu$ ( $t_{\text {TOT-1 }}, t_{\text {TOT }}$ ) (see equation (4.24)) will be smaller than the one corresponding to simple LMS which means faster convergence. But for the reasons discussed in Section 4.2.3 SLMS will still be slower than MLMS. This can be seen in practice, as shown in the following section.

\subsection{EXPERIMENTAL RESULTS}

First we use some simulated data to see the performance of SLMS, MLMS end MDLMS. For this purpose white Gaussian noise is added to a straight-line section and the data are represented by the following ${ }^{1}$ equation:

$$
\operatorname{RDATA}(\mathrm{I})=\mathrm{RNG}(\mathrm{I})+1.6 * I
$$

\footnotetext{
${ }^{1}$ Fortran-like.
} 


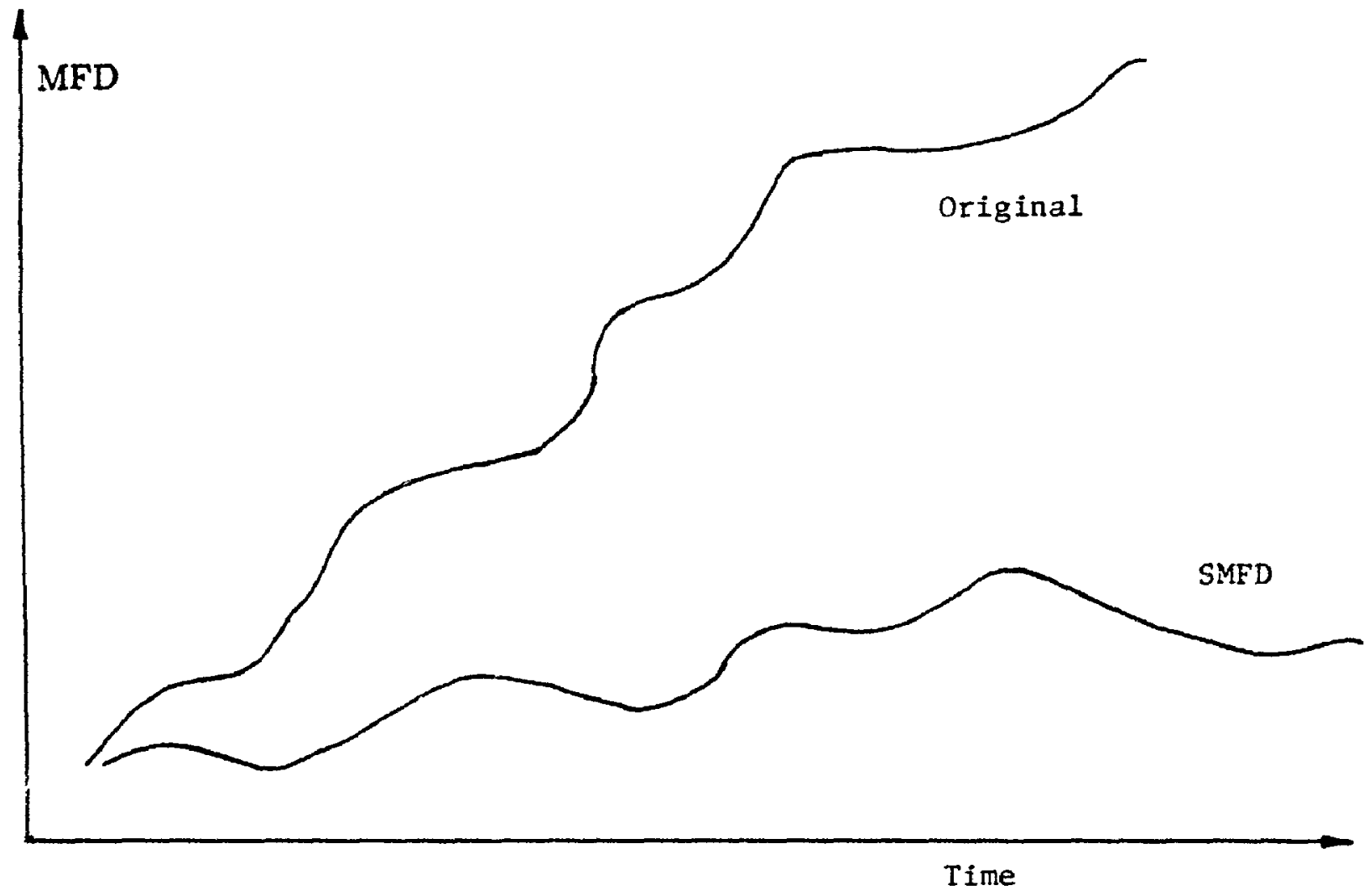

Figure 4.3: Original and new curves (SMFD) obtained as indicated in 4.2.3 
where RNG is the additive white Gaussian noise. Here, since a straight line section is assumed, equations (4.13a) and (4.13b) apply. We want to see how using different but very small amounts of data $(k: 3 \leq k \leq 10)$ will affect $A_{0}, A_{1}, A_{00}$, and $A_{11}$. This will affect the performance of SLMS but not that of MLMS as has been explained in the previous sections. From equation (4.26) having used $T=0.4$ we have $\omega_{1}=4$. Now when the variance of the noise sequence RNG is equal to 2.2, then using 5-6 data points we get $A_{1}=2.86$. If we use 8-10 data points we get $A_{1}=3.4$. Figures $4.4 \mathrm{a}$ and $4.4 \mathrm{~b}$ represent the performance of MLMS and SLMS respectively for the worst case estimate $A_{1}=2.86$. MLMS reaches steady-state within 15 iterations, while SLMS needs 34-40 iterations. It should be also noted that the average MSE after the 15th iteration is 3.34 for MLMS while it is 4.80 for SLMS after the 35th iteration. Hence in order to get a smaller error, a small $r \mu$ should be used in SLMS and this will make it even slower ( 70 iterations). in Figure 4.4c, which has a different vertical scale, one can see clearly this difference. In Figures $4.5 \mathrm{a}$ and $4.5 \mathrm{~b}$, the performance of the same algorithms is shown for $A_{1}=3.4$. It should be noted here that while the two algorithms converge within 13 (MLMS) and 35 (SLMS) iterations, now the average steady state MSE are close to each other (3.4 for MLMS, 3.75 for SLMS). From all these figures one conclusion is that as $A_{1}$ gets closer to $\omega_{1}$, the two algorithms converge to the same performance in terms of MSE. However, the more important conclusion is that MLMS does not need a good estimate of $\omega_{1}$ in order to converge fast, while SLMS is very sensitive to the error $\left(\omega_{1}-A_{1}\right)$ as has already been pointed out in Section 4.2.2. It was also observed that MDLMS has very similar serformance in the simulations to the performance of MLMS. 


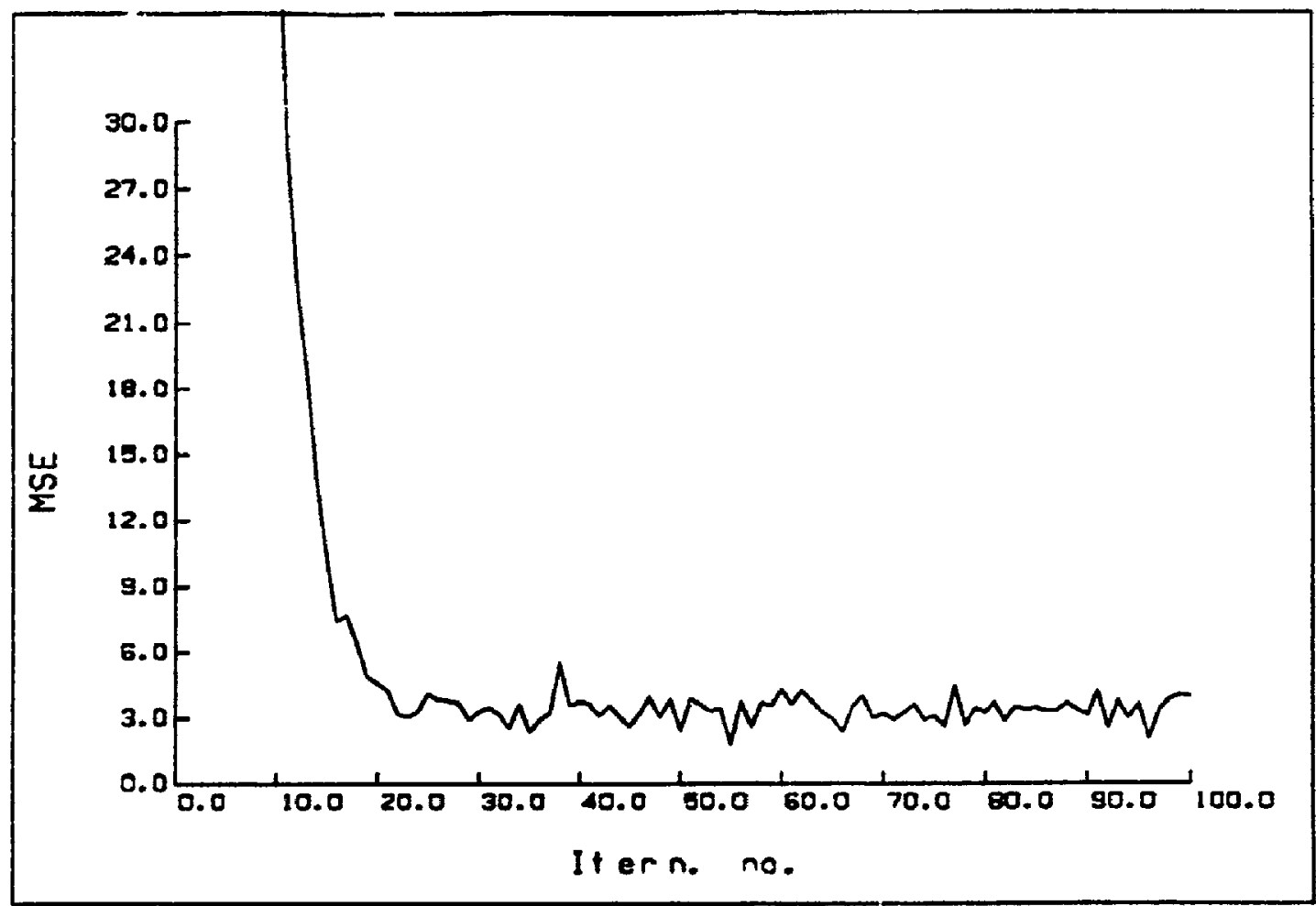

(a)

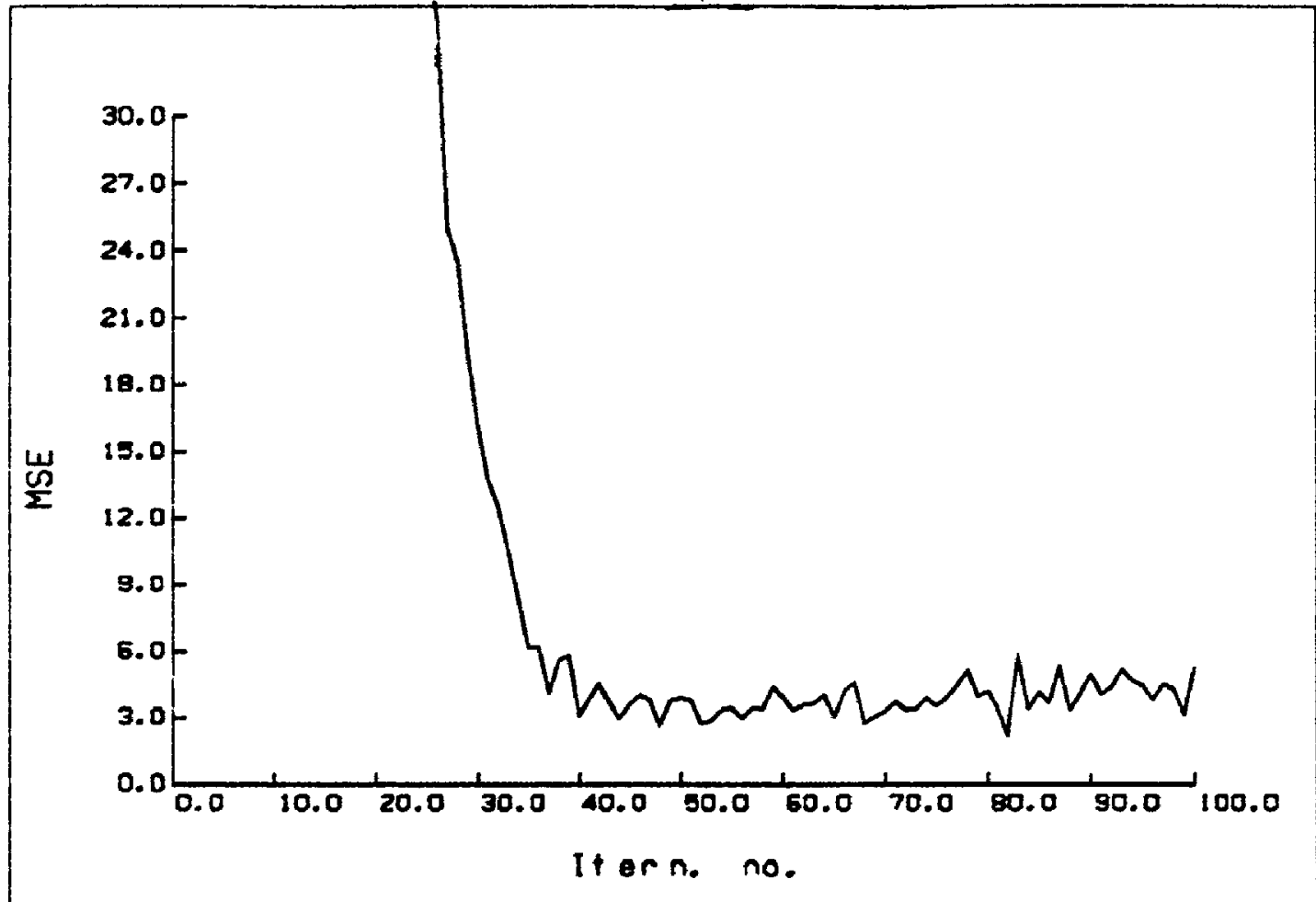

(b)

Figure 4.4: (a): MLMS with MFD-straight line and noise; (b): SLMS $*^{\circ} \cdot h$ MFD=straight line and noise 


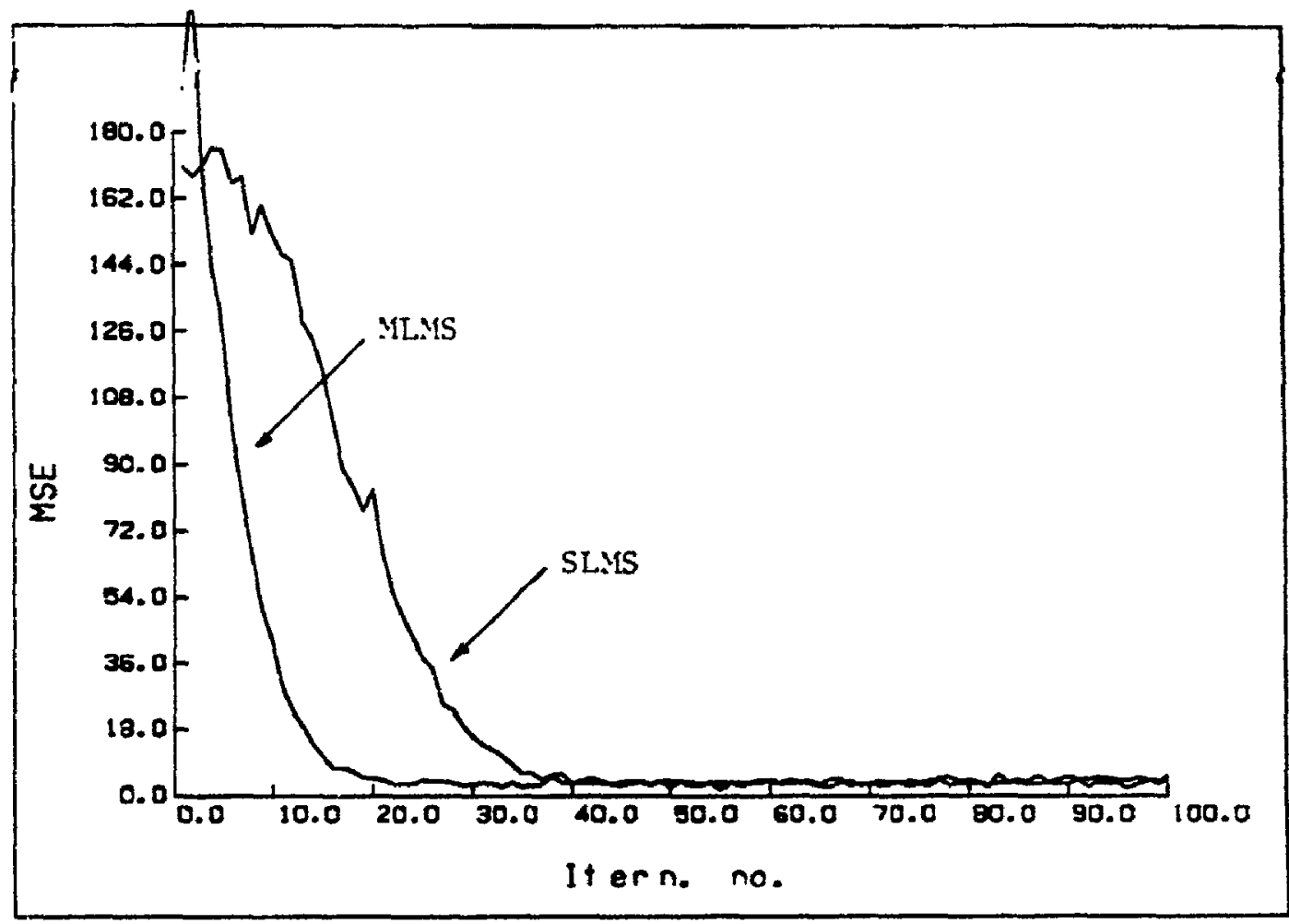

(c)

Figure 4.4 (continued): (c) MLMS compared to SLMS from Figures 4.4(a) and 4.4(b) 


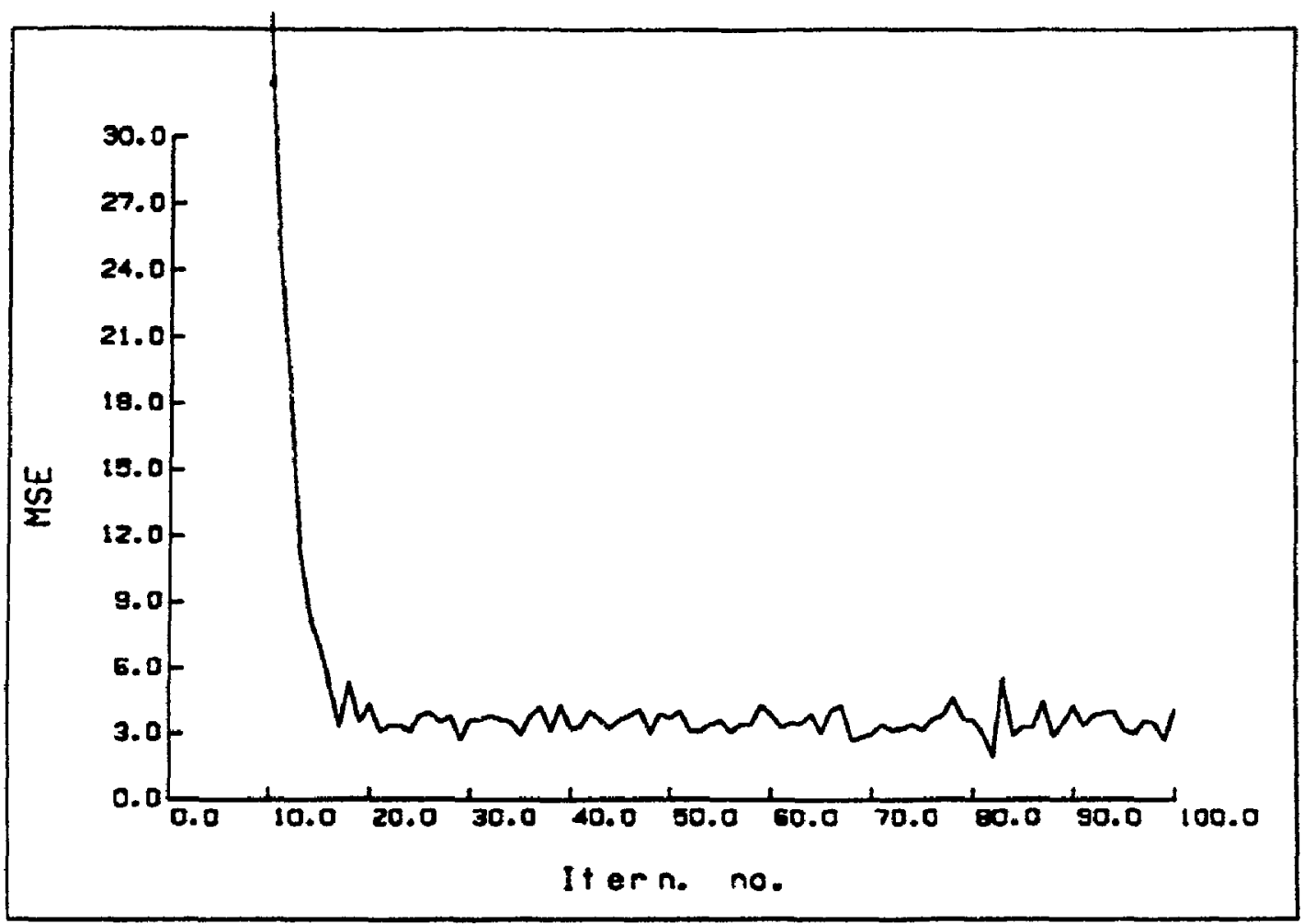

(a)

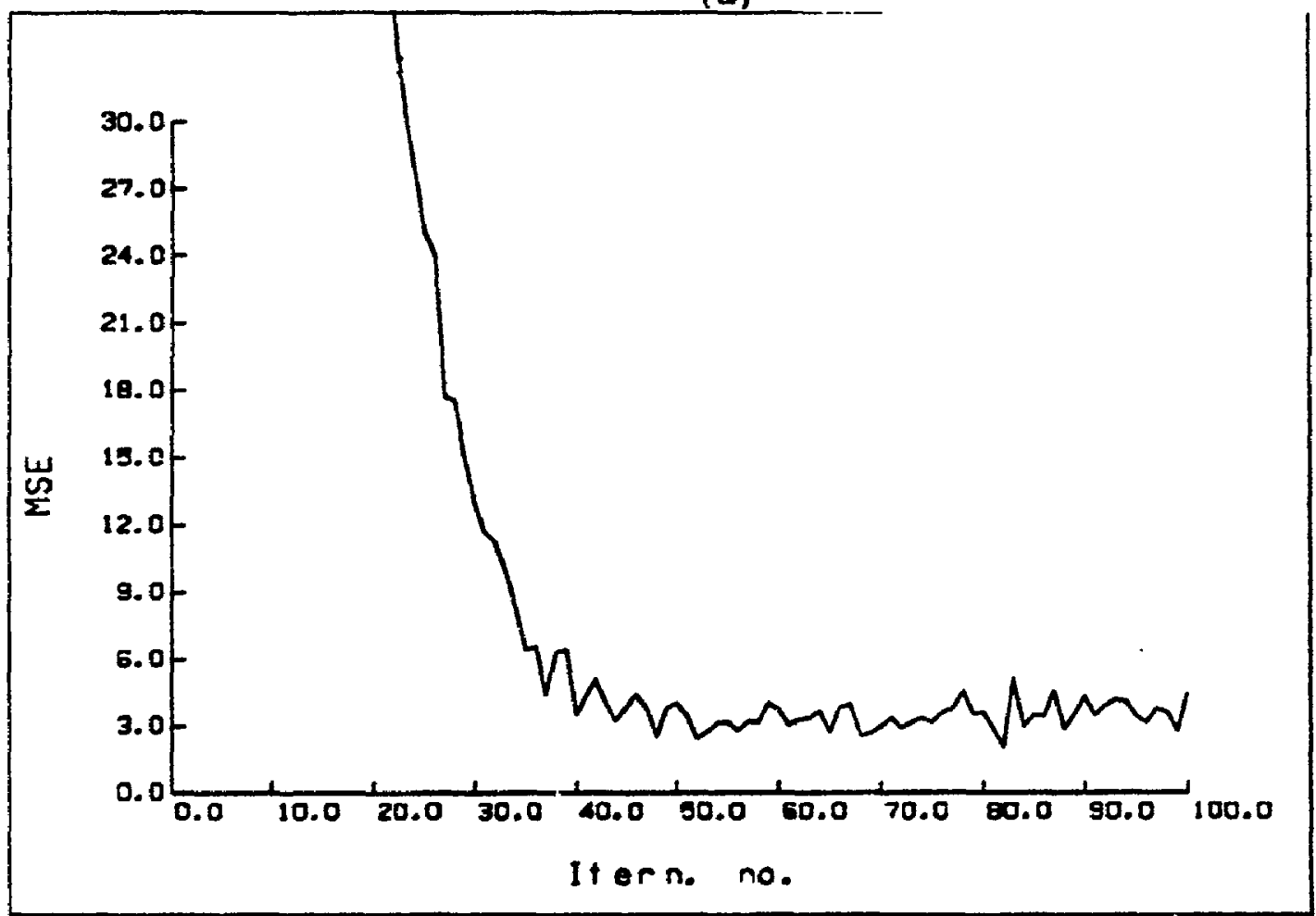

(b)

Figure 4.5: (a): MLMS with MFD=straight line and noise; (b): SLMS with $\mathrm{MFD}=$ straight line and noise 
Using the experimental set-up described in Section 2.5 (Figure 2.6) some real data files were obtained for various frequency hopping patterns. The system was set to hop between two frequencies with dwell time equal to $70 \mu \mathrm{sec}$, and to record the counter output every 5 msec. The VCO was heated using a heat gun from $20^{\circ} \mathrm{C}$ to $28^{\circ} \mathrm{C}$ while simultaneously hopping between two frequencies. That is how the two data files "var505" and "var175" were produced. Each data file contains around 14000 measurements. One out of every 20 data points was used to produce Figures 4.6a and 4.6b. For Figure 4.6a the difference between the two hopping frequencies was $f_{d}=5 \mathrm{MHz}$ while for Figure $4.6 \mathrm{~b}$ it was $f_{d}=20 \mathrm{MHz}$.

In Figure 4.6a one can even distinguish (after 280 time units) a slight systematic, almost sinusoidal, variation possibly due to the coupling described in the beginning of this chapter, superimposed on the long-term drift due to temperature variation. Figure 4.6c shows the performance of MLMS using the "var505" data file and adding the same amount of noise as in Figures 4.4 and 4.5. The difference here is that instead of only one $A_{1}$ we use two different values because the MFD is not a straight line any more. One $A_{1}$ is used for the first 35 iterations and another one for the rest. One can see from Figure 4.6c that MLMS still converges within 12-15 iterations. The performance of SLMS is again the same as in Figures 4.4 and 4.5.

Concluding this chapter we note the following:

1. A modified form of the LMS algorithm has been outlined starting from a formulation similar to the orthogonalized LMS or SER-Kalman algorithms, where an estimate of $[E\{\hat{R}\}]^{-1}$ or of $[\hat{R}]^{-1}$ is used.

2. The validity of approximating MDLMS to the useful form of MLMS is directly related to the range of values for which $\omega_{1} T$ obeys inequality (4.18a). This means that since $\omega_{1}$ is very small for our system, $T$ can be several times greater than the dwell time. This can give us a flexibility in the design of the 


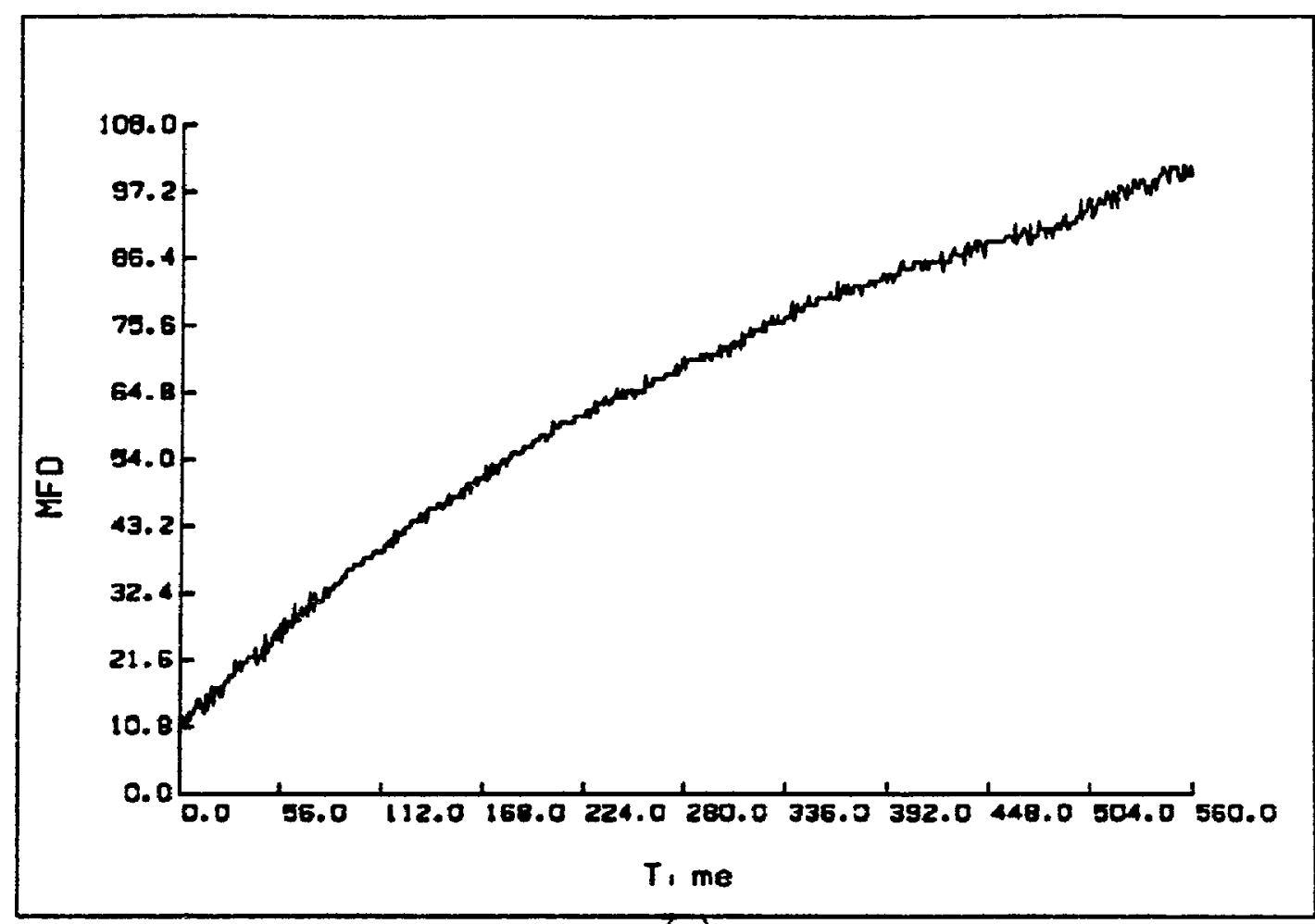

(a)

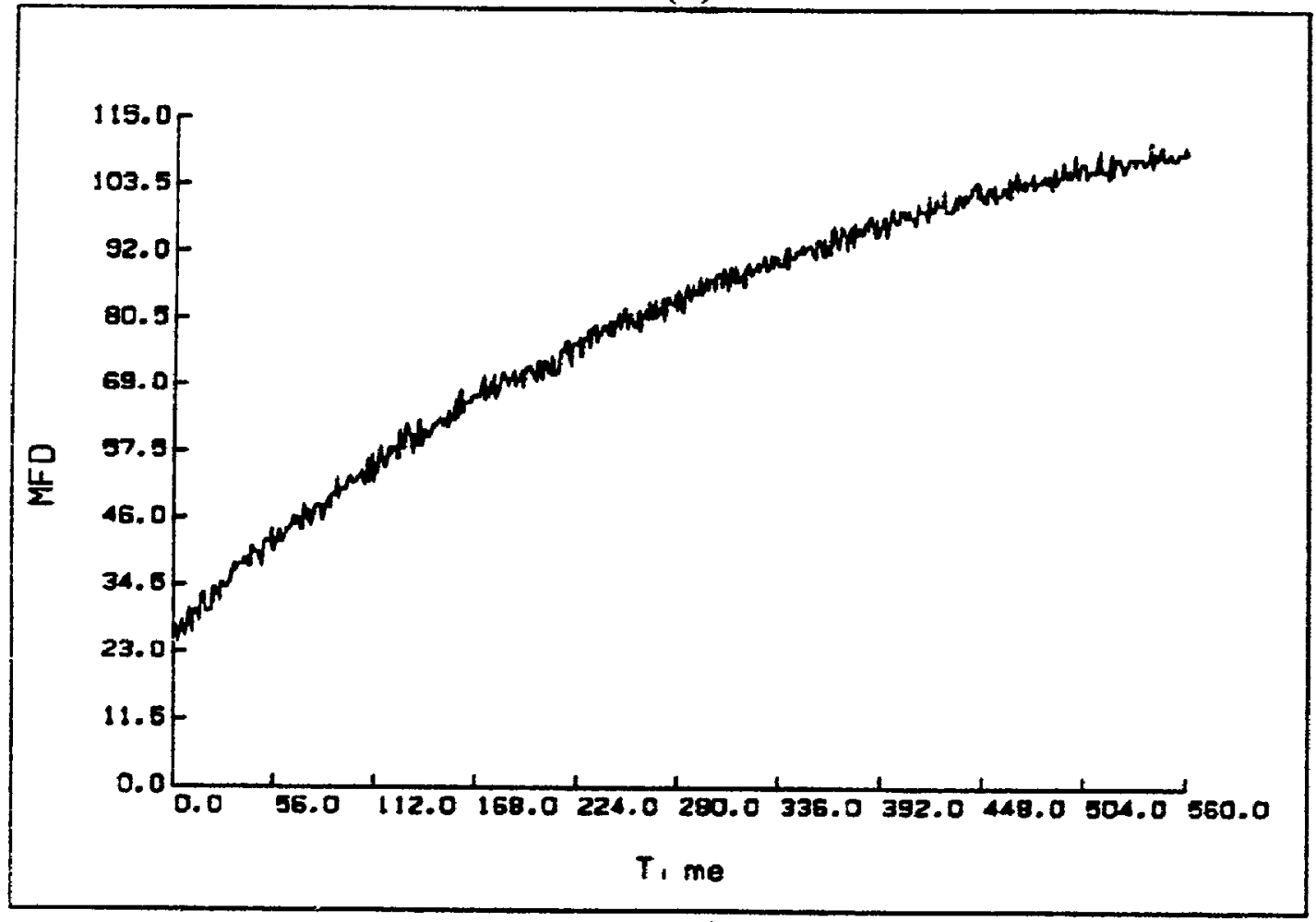

(b)

Figure 4.6: (a): "Var505" real MFD; (b): "Var175" real MFD 


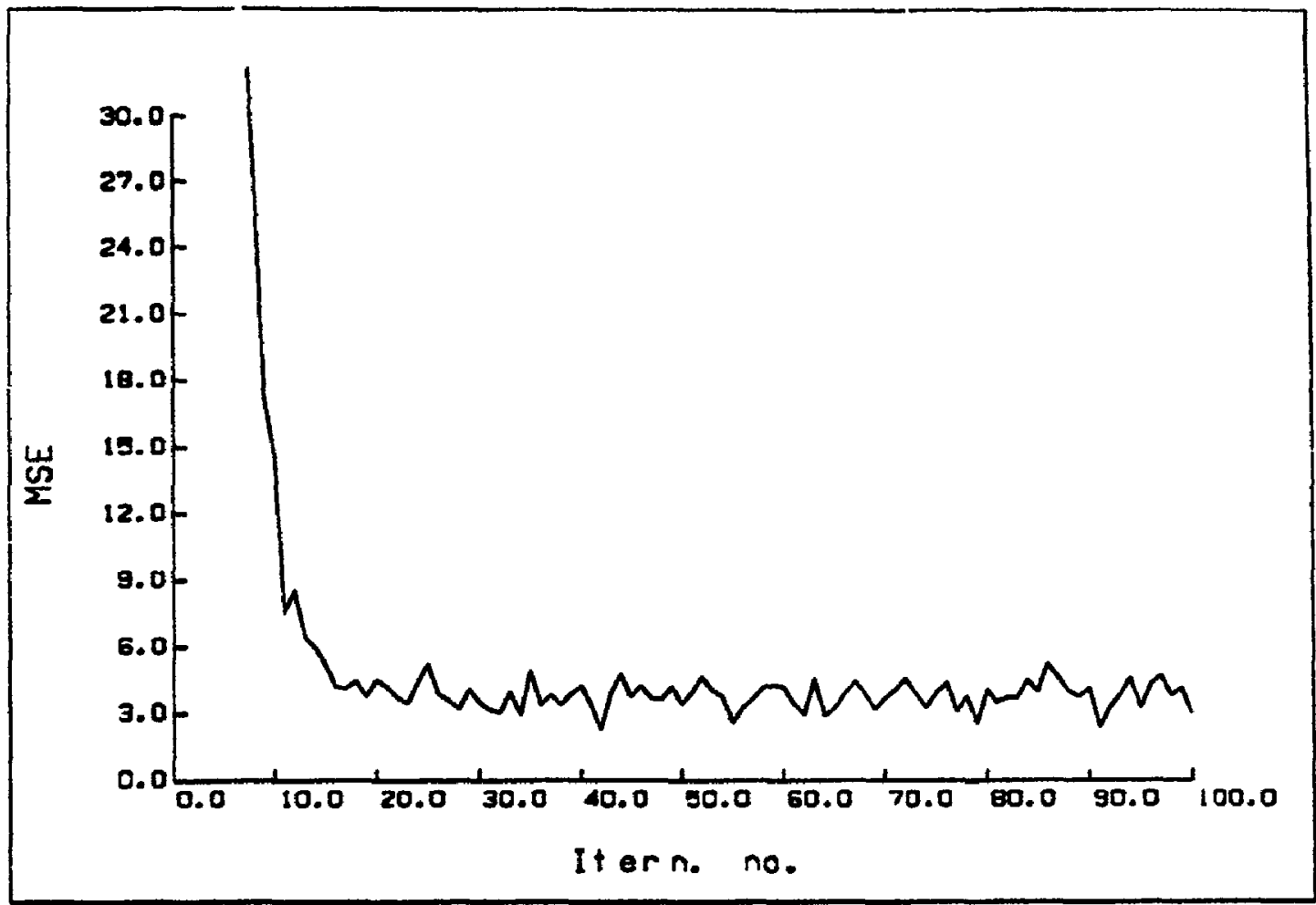

(c)

Figure 4.6 (continued): (c): MLMS with "Var505" data file and noise 
frequency-hopping strategy by transmitting information plus a small periodic burst of a "training sequence" consisting of several frequencies "transmitted" for 70-100 $\mu \mathrm{sec}$ each in order to be counted. This can increase the hopping speed of the synthesizer by 5 to 10 times because since we do not count during the information transmission, the dwell time can be minimized to several $\mu$ sec.

In general, it has been shown that by using adaptive algorithms for frequency correction, independence of dwell time and frequency counting resolution can be established, implying an increase of the hopping speed of the system. 


\section{APPENDIX 4.1}

CALCULATION OF MAXIMUM IIKELIHOOD ESTIMATES FOR $\omega_{0}$, $\omega_{1}$

We assume that for a straight-line portion of data we have:

$$
x(i)=\omega_{0}+\omega_{i} T \cdot i+v(i T)
$$

We want to derive ML estimates for $\omega_{0}, \omega_{1}$ ( $\hat{\omega}_{0}, \hat{\omega}_{1}$ respectively). According to equation (4.9):

$$
\sigma_{x, i-j}^{2}=\sigma_{x, 0}^{2} \cdot \delta(i-j)
$$

The MLE criterion is ([Nahi 69], p.197):

$$
\min _{\omega_{0}, \omega_{1}} \sum_{i=0}^{k}\left[x(i)-\omega_{0}-\omega_{1} \cdot i T\right]^{T} \cdot L^{-1}(i, i)\left[x(i)-\omega_{0}-\omega_{1} i T\right]
$$

where

$$
L(i, i) \triangleq \sigma_{x, i-i}^{2}=\sigma_{x, 0}^{2}
$$

In equation (4.29) we can omit $L^{-1}(i, i)$ because as seen from equation (4.30) it has the same value for all $i$, and since $x(i)-\omega_{0}-\omega_{1} i T$ is not a vector, equation (4.29) takes the form: 


$$
\min _{\omega_{0}, \omega_{i}} \sum_{i=0}^{k}\left[x(i)-\omega_{0}-\omega_{1} T i\right]^{2}
$$

which gives the following two equations:

$$
\begin{gathered}
\sum_{i=0}^{k}\left[x(i)-\hat{\omega}_{0}-\hat{\omega}_{1} T i\right]=0 \\
T \sum_{i=0}^{k} i\left[x(i)-\hat{\omega}_{0}-\hat{\omega}_{1} T i\right]=0 .
\end{gathered}
$$

Now we just have to solve equations (4.32) and (4.33) for $\hat{\omega}_{0}, \hat{\omega}_{1}$. Equation (4.32) gives:

$$
\sum_{i=0}^{k} x(i)-(k+1) \hat{\omega}_{0}-\hat{\omega}_{1} T \frac{k(k+1)}{2}=0
$$

Similarly from (4.33)

$$
\sum_{i=0}^{k} i x(i)-\frac{k(k+1)}{2} \hat{\omega}_{0}-\hat{\omega}_{1} T \frac{k(k+1)(2 k+1)}{6}=0 .
$$

Multiplying equation (4.34) by $-\frac{2 k+1}{3}$ we get:

$$
-\frac{2 k+1}{3} \cdot \sum_{i=0}^{k} x(i)+\frac{(k+1)(2 k+1)}{3} \hat{\omega}_{0}+\hat{\omega}_{1} T \frac{k(k+1)(2 k+1)}{6}=0
$$

and adding this to equation (4.35) we get:

$$
\left[\frac{(k+1)(2 k+1)}{3}-\frac{k(k+1)}{2}\right] \hat{\omega}_{0}=\frac{2 k+1}{3} \cdot \sum_{i=0}^{k} x(i)-\sum_{i=0}^{k} i x(i)
$$


which leads to:

$$
\hat{\omega}_{0}=\frac{2(2 k+1)}{(k+1)(k+2)} \cdot \sum_{i=0}^{k} x(i)-\frac{6}{(k+1)(k+2)} \cdot \sum_{i=0}^{k} i x(i)
$$

If we now put the value of $\hat{\omega}_{0}$ given by equation (4.36) into equation (4.35) we get:

$$
\hat{\omega}_{1} T=\frac{2}{k(k+1)} \sum_{i=0}^{k} x(i)-\frac{2}{k} \hat{\omega}_{0}
$$

Let us now calculate the means and variances of the random variables $\hat{\omega}_{0}$ and $\hat{\omega}_{1}$. Although this can be done by straightforward manipulations using equations (4.36) and (4.37) it is preferred to use the more elegant method of $\$ 8.4$ in [Nahi 69]. We briefly present the method and after that we apply it to the particular application at hand.

When the observetions are linear functions of the parameters to be estimated, then we have the following model for $x(i)$ and the following equations are true:

$$
x(i)=g^{T}(i) \cdot \omega+v(i) \quad i=1, \ldots k
$$

where $g(i)$ and $\omega$ are $n$-vectors and $x(i), v(i)$ are scalars. 


$$
\begin{aligned}
& x_{k}=[x(0), x(1), \ldots, x(k)]^{T} \\
& G_{k}=\left[\begin{array}{c}
g^{T}(0) \\
g^{T}(1) \\
\vdots \\
g^{T}(k)
\end{array}\right\} \\
& v_{k}=[v(0), v(1), \ldots, v(k)]^{T} .
\end{aligned}
$$

Hence $x_{k}$ and $v_{k}$ are $(k+1)$-vectors while $G_{k}$ is a $(k+1) \times n$ matrix. It is proved in the same reference ([Nahi 69]) that:

$$
E\left\{\hat{\boldsymbol{\omega}}_{k}\right\}=\boldsymbol{\omega}
$$

and

$$
E\left\{\left(\omega-\hat{\omega}_{k}\right)\left(\omega-\omega_{k}\right)^{T}\right\}=P_{k}
$$

where

$$
P_{k}=\left[G_{k}^{T} L_{k}^{-1} G_{k}\right]^{-1}
$$

and

$$
L_{k}=\operatorname{diag}\left(\sigma_{0}^{2}, \sigma_{1}^{2}, \ldots, \sigma_{k}^{2}\right)
$$

In our application we have: 


$$
\begin{gathered}
\omega=\left[\omega_{0}, \omega_{1}\right]^{T} \\
\hat{\omega}_{k}=\left[\hat{\omega}_{0}(k), \hat{\omega}_{1}(k)\right]^{T} \\
G_{k}=\left[\begin{array}{cc}
1 & 0 \\
1 & 1 \\
1 & 2 \\
\vdots & \vdots \\
1 & k
\end{array}\right],
\end{gathered}
$$

the transpose of which is

$$
G_{k}^{T}\left[\begin{array}{ccccc}
1 & 1 & 1 & \ldots & 1 \\
0 & 1 & 2 & \ldots & k
\end{array}\right]
$$

and

$$
L_{k}=\operatorname{diag}\left(\sigma_{x, 0}^{2}, \ldots, \sigma_{x, 0}^{2}\right)
$$

Using equation (4.44), equation (4.41) gives:

$$
\begin{aligned}
E\left\{\left(\begin{array}{c}
\omega_{0}-\hat{\omega}_{0}(k) \\
\omega_{1}-\hat{\omega}_{1}(k)
\end{array}\right)\right. & \left.\cdot\left[\omega_{0}-\hat{\omega}_{0}(k), \omega_{1}-\hat{\omega}_{1}(k)\right]\right\}= \\
& =E\left\{\begin{array}{c}
{\left[\omega_{0}-\hat{\omega}_{0}(k)\right]^{2}} \\
\cdot
\end{array}=\left[\omega_{1}-\hat{\omega}_{1}(k)\right]^{2}\right\}
\end{aligned}
$$

Equation (4.42) gives: 


$$
\begin{aligned}
& P_{k}=\sigma_{x, 0}^{2}\left[\left(\begin{array}{ccccc}
1 & 1 & . & \ldots & 1 \\
0 & 1 & 0 & \ldots & k
\end{array}\right)\left(\begin{array}{ccccc}
1 & 0 & 0 & \ldots & \\
0 & 1 & 0 & \ldots & \\
& \vdots & & \ldots & 1
\end{array}\right)\left(\begin{array}{cc}
1 & 0 \\
1 & 1 \\
\cdot & 2 \\
\cdot & \cdot \\
\cdot & . \\
1 & k
\end{array}\right)\right]^{-1} \\
& =\sigma_{x, 0}^{2}\left[\left(\begin{array}{ccccc}
1 & 1 & \cdot & \ldots & 1 \\
0 & 1 & 2 & \ldots & k
\end{array}\right)\left(\begin{array}{cc}
1 & 0 \\
1 & 1 \\
\cdot & 2 \\
\cdot & \cdot \\
\cdot & \cdot \\
1 & k
\end{array}\right)\right]^{-1}=
\end{aligned}
$$

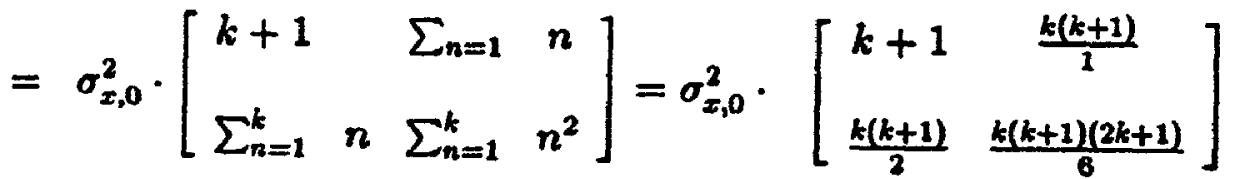

From equations (4.47) and (4.48) we get:

$$
\begin{gathered}
D=\text { Determinant }=\frac{(k+1)^{2} k(k+2)}{12} \\
E\left\{\left[\omega_{1}-\hat{\omega}_{1}(k)\right]^{2}\right\}=\sigma_{x, 0}^{2} \cdot \frac{k+1}{D}=\sigma_{x, 0}^{2} \cdot \frac{12}{k(k+1)(k+2)} \\
E\left\{\left[\omega_{0}-\hat{\omega}_{0}(k)\right]^{2}\right\}=\sigma_{x, 0}^{2} \cdot \frac{k(k+1)(2 k+1)}{6} \cdot D^{-1}=\frac{2 \sigma_{x, 0}^{2} \cdot(2 k+1)}{(k+1)(k+2)}
\end{gathered}
$$

Finally, it can be observed from equations (4.36) and (4.37) that recursive formulas can be developed which will enable us to estimate $\hat{\omega}_{0}(k+1), \hat{\omega}_{1}(k+1)$ based only on $\hat{\omega}_{0}(k), \hat{\omega}_{1}(k)$ and the most recent additional measurement. It can be shown that this io due to the linear dependence of data $x(i)$ on the vector $\omega$. This conclusion 
is very important because, choosing a window of finite width $k$ for the data, we can update the estimates $\dot{\omega}_{0}(k+1), \hat{\omega}_{1}(k+1)$ with a small amount of calculation regardless of how lerge $k$ is. This means that at steady state, with the same amount of calculation we can make variances (4.49) and (4.50) as small as we want, provided that the model of equation (4.27) is valid for the group of data used in a particular window of width $k$. 
REASONS FOR USING $[E\{\hat{R}(n)\}]^{-1}$ INSTEAD OF $[\hat{R}(n)]^{-1}$ IN EQUATION (4.5)

The following model can be used ([Eleftheriou 85]):

$$
\hat{R}(n)=E\{\hat{R}(n)\}+\tilde{R}(n)
$$

where $\tilde{R}(n)$ is a perturbation matrix whose elements are zero-mean random variables. Equation (4.51) gives:

$$
\hat{R}(n)=E\{\hat{R}(n)\} \cdot\left[I+(E\{\hat{R}(n)\})^{-1} \cdot \tilde{R}(n)\right]
$$

Hence:

$$
[\hat{R}(n)]^{-1}=\left[I+(E\{\hat{R}(n)\})^{-1} \cdot \tilde{R}(n)\right]^{-1} \cdot(E\{\hat{R}(n)\})^{-1}
$$

where $I$ is the identity matrix. We can assume that the following is true:

$$
\left\|(E\{\hat{R}(n)\})^{-1} \cdot \tilde{R}(n)\right\|<1
$$

where $\|\cdot\|$ represents a matrix norm. Equation (4.53) is a reasonable assumption especially for systems with slowly-varying autocorrelation matrix $R(n)$. This is the case for the application at hand since $\omega_{1} T$ is several times smaller than 1 as has been justified in Chapter 4. A justification for equation (4.53) is given below. We have: 


$$
\begin{aligned}
\hat{R}(n) & =\sum_{k=1}^{n} \lambda^{n-k} R(k)+\tilde{R}(n) \\
& \simeq \frac{1}{1-\lambda} R(n)+\tilde{R}(n)
\end{aligned}
$$

and from equation (4.51) we have:

$$
E\{\hat{R}(n)\} \simeq \frac{1}{1-\lambda} R(n)
$$

which gives:

$$
(E\{\hat{R}(n)\})^{-1} \cdot \hat{R}(n) \simeq(1-\lambda) \cdot[R(n)]^{-1} \cdot \tilde{R}(n)
$$

The norm of equation (4.56) obviously can be taken to be less than 1 . Using equation (4.53), (4.52) becomes:

$$
[\hat{R}(n)]^{-1} \simeq\left[I-(E\{\hat{R}(n)\})^{-1} \cdot \tilde{R}(n)\right] \cdot(E\{\dot{R}(n)\})^{-1}
$$

or

$$
[\hat{R}(n)]^{-1} \simeq(E\{\hat{R}(n)\})^{-1}-(E\{\hat{R}(n)\})^{-1} \cdot \tilde{R}(n) \cdot(E\{\hat{R}(n)\})^{-1}
$$

In equation (4.57) the last term of the RHS represents second-order statistics and hence can be omitted as being very small. 


\section{APPENDIX 4.3}

\section{DERIVATION OF THE ELEMENTS OF $[E\{\hat{R}(k)\}]^{-1}$}

If $q_{i j}$ are the elements of $E\{\hat{R}(k)\}$, then using equations (4.6) to (4.9) we get:

$$
\begin{aligned}
q_{11} & =\sum_{s=s_{1}}^{n} \lambda^{n-\bullet} \cdot E\left(x_{1,} \cdot x_{1 s}\right)= \\
& \left.=\sum_{s=s_{1}}^{n} \lambda^{n-1 \cdot} \overline{\left[\hat{\omega}_{0, s-1}^{2}\right.}+2 T(s-1) \overline{\hat{\omega}_{0, s-1} \cdot \hat{\omega}_{1, s-1}}+T^{2}(s-1)^{2} \overline{\hat{\omega}_{1, s-1}}+\sigma_{x, 0}^{2}\right] \\
& =\sum \lambda^{n-s} \overline{\left[\hat{\omega}_{0, \ell-1}^{2}\right.}+2 T s \overline{\hat{\omega}_{0, s-1} \cdot \hat{\omega}_{1, s-1}}-2 T \overline{\hat{\omega}_{1, \ell-1} \cdot \hat{\omega}_{0, s-1}}+ \\
& \left.+T^{2} s^{2} \overline{\hat{\omega}_{1, \ell-1}^{2}}+T^{2} \overline{\hat{\omega}_{1, \ell-1}^{2}}-2 T^{2} s \overline{\hat{\omega}_{1, s-1}^{2}}+\sigma_{x, 0}^{2}\right]
\end{aligned}
$$

Now we define:

$$
\begin{gathered}
F_{i}(n) \triangleq \sum_{s=s_{i}}^{n} \lambda^{n-s} \overline{\hat{\omega}_{0,2-1}^{2}}+2 T \sum_{s=s_{i}}^{n} \lambda^{n-*} \cdot s \cdot \overline{\hat{\omega}_{0, s-1} \cdot \hat{\omega}_{1,8-1}} \\
+T^{2} \sum_{s=s_{i}}^{n} \lambda^{n-s} \cdot s^{2} \overline{\hat{\omega}_{1, s-1}^{2}} \\
C_{1, i}(n) \triangleq T \sum_{s=s_{i}}^{n} \lambda^{n-s} \overline{\hat{\omega}_{0, s-1} \cdot \hat{\omega}_{1, s-1}} \\
C_{2, i}(n) \triangleq T^{2} \sum_{s=0, i}^{n} \lambda^{n-s} \cdot s \cdot \overline{\hat{\omega}_{1, s-1}^{2}}
\end{gathered}
$$




$$
C_{3, i}(n) \triangleq T^{2} \sum_{s=\Omega_{1}} \lambda^{n-8} \overline{\bar{\omega}_{1, \Omega-1}^{2}}
$$

The horizontal bar signifies the $E\{\}$ operator. Using equations (4.59) to (4.62), equation (4.58) becomes:

$$
q_{11}(n)=F_{1}(n)-2\left(C_{1,1}(n)+C_{2,1}(n)\right)+C_{3,1}(n)+\sigma_{0}^{2}
$$

Now we assume that three successive estimates of both $\omega_{0}$, and $\omega_{1}$ are almost equal:

$$
\left.\begin{array}{l}
\hat{\omega}_{0, \&-1} \simeq \hat{\omega}_{0, \&-2} \simeq \hat{\omega}_{0, \&-3} \\
\hat{\omega}_{1, \&-1} \simeq \hat{\omega}_{1, \ell-2} \simeq \hat{\omega}_{1, \&-3}
\end{array}\right\}
$$

Equations (4.64) are referred to throughout Chapter 4 as the "restrictive assumptions". These assurnptions are reasonable when there are no abrupt changes in the MFD, as in our application. Because the data used to produce the example $\hat{\omega}_{0,0-1}$ and $\hat{\omega}_{0, s-2}$ differ by only one data point,

$$
\begin{aligned}
q_{12} & =\sum_{s=s,}^{n} \lambda^{n-s} E\left\{x_{1} \cdot x_{2 s}\right\}= \\
& =\sum_{s=\infty 2}^{n} \lambda^{n-s}\left[\overline{\hat{\omega}_{0, s-1} \cdot \hat{\omega}_{0, s-2}}+T(s-1) \overline{\hat{\omega}_{0, s-2} \hat{\omega}_{1, \ell-1}}+\right. \\
& \left.+T(s-2) \overline{\hat{\omega}_{1, s-2} \cdot \hat{\omega}_{0, s-1}}+T^{2}(s-1)(s-2) \overline{\hat{\omega}_{1, \Omega-1} \cdot \hat{\omega}_{1, s-2}}\right]
\end{aligned}
$$

and using equation (4.64) we get: 


$$
q_{12}(n)=\sum_{s=\varepsilon_{2}}^{n} \lambda^{n-\infty}\left[\overline{\hat{\omega}_{0, \varepsilon-1}^{2}}+T(2 s-3) \overline{\hat{\omega}_{1, \varepsilon-1} \cdot \bar{\omega}_{0, \varepsilon-1}}+T^{2}(s-1)(s-2) \overline{\hat{\omega}_{1, s-1}^{2}}\right]
$$

and using again equations (4.59) to (4.62)

$$
q_{12}(n)=F_{2}(n)-3\left(C_{1,2}+C_{2,2}\right)+2 C_{3,2}
$$

Similarly:

$$
\begin{aligned}
q_{13}(n) & \simeq \sum_{s=s s}^{n} \lambda^{n-1}\left[\overline{\hat{\omega}_{0, s-1}^{2}}+2 T s \overline{\hat{\omega}_{1, \ell-1} \cdot \hat{\omega}_{0,8-1}}-4 T \overline{\hat{\omega}_{0, \ell-1} \cdot \hat{\omega}_{1, \ell-1}}\right. \\
& \left.+T^{2} s^{2} \overline{\hat{\omega}_{1, s-1}^{2}}-4 T^{2} s \overline{\hat{\omega}_{1, \&-1}^{2}}+3 T^{2} \overline{\hat{\omega}_{1,8-1}^{2}}\right]
\end{aligned}
$$

which gives:

$$
q_{13}(n)=F_{3}(n)-4\left(C_{1,3}(n)+C_{2,3}(n)\right)+3 C_{3,3}(n)
$$

Using the same procedure we can get the rest of equations (4.10) as given in the text. It should be noted here that $s_{1}=1, s_{2}=2, s_{3}=3$. The different lower limits for the series in each element $q_{i j}$ is due to the fact that the signal vector is empty at the beginning and is full 3 iterations later. This results in having $F_{1} \neq F_{2} \neq F_{3}$. But depending on the value of $\lambda$ this is true for a few iterations in the beginning, because (from the nature of $\hat{R}(n)$ ) the effect of the distant past is highly "attenuated". Hence it is obvious for example, that for $n=10, \lambda=0.70$ it will not make any differcnce if the lower limit of the series is 0 or 3 because these first terms are attenuated by $\lambda^{10}$ and $\lambda^{7}$ respectively which will give very small 
numbers compared to the 4-5 more recent terms. For these reasons the expressions for $q_{i j}$ can be given by equations (4.11). To calculate the inverse of the symmetric matrix with elements $q_{i j}, i, j=1,2,3$ we have first to calculate the minors $D_{i j}$ and the determinant $D_{0}$. The minor $D_{11}$ is given by:

$$
\begin{aligned}
D_{11} & =q_{22} q_{33}-q_{23}^{2}=\left[\sigma_{0}^{2}+F-4\left(C_{1}+C_{2}\right)+4 C_{3}\right] \cdot\left[\sigma^{2}+F-6\left(C_{1}+C_{2}\right)+9 C_{3}\right] \\
& -\left[F-5\left(C_{1}+C_{2}\right)+6 C_{3}\right]^{2} .
\end{aligned}
$$

After some algebraic manipulations we get:

$$
\left.\begin{array}{l}
D_{11}=\left(\sigma_{0}^{2}\right)^{2}+F\left(2 \sigma_{0}^{2}+C_{3}\right)-10 \sigma_{0}^{2}\left(C_{1}+C_{2}\right)-\left(C_{1}+C_{2}\right)^{2}+13 \sigma_{0}^{2} C_{3} . \\
D_{12}=F\left(\sigma_{0}^{2}+2 C_{3}\right)-3 \sigma_{0}^{2}\left(C_{1}+C_{2}\right)-2\left(C_{1}+C_{2}\right)^{2}+2 \sigma_{0}^{2} C_{3} \\
D_{13}=F\left(C_{3}-\sigma_{0}^{2}\right)+4 \sigma_{0}^{2}\left(C_{1}+C_{2}\right)-\left(C_{1}+C_{2}\right)^{2}-3 \sigma_{0}^{2} C_{3} \\
D_{22}=F\left(2 \sigma_{0}^{2}+4 C_{3}\right)+\left(\sigma_{0}^{2}\right)^{2}-8 \sigma_{0}^{2}\left(C_{1}+C_{2}\right)-4\left(C_{1}+C_{2}\right)^{2}+10 \sigma_{0}^{2} C_{3} \\
D_{23}=F\left(\sigma_{0}^{2}+2 C_{3}\right)-5 \sigma_{0}^{2}\left(C_{1}+C_{2}\right)-2\left(C_{1}+C_{2}\right)^{2}+6 \sigma_{0}^{2} C_{3} \\
D_{33}=\left(\sigma_{0}^{2}\right)^{2}+F\left(2 \sigma_{0}^{2}+C_{3}\right)-6 \sigma_{0}^{2}\left(C_{1}+C_{2}\right)-\left(C_{1}+C_{2}\right)^{2}+5 \sigma_{0}^{2} C_{3} .
\end{array}\right\}
$$

where $D_{i j}$ terms are obtained in a similar manner as $D_{11}$. Finally, we get for the determinant $D_{0}$ :

$$
\begin{aligned}
D_{0} & =3\left(\sigma_{0}^{2}\right)^{2} F+\left(\sigma_{0}^{2}\right)^{3}+12\left(\sigma_{0}^{2}\right)^{2}\left(C_{1}+C_{2}\right) \\
& +6 \sigma_{0}^{2}\left[F \cdot C_{3}-\left(C_{1}+C_{2}\right)^{2}\right]
\end{aligned}
$$


We observe now that the term $F C_{3}-\left(C_{1}+C_{2}\right)^{2}$ appears not only in $D_{0}$ but also in all minors with dufferent coefficients. For example in $D_{22}$ the coel..cient is 4 . In the last part of this Appendix we prove now that:

$$
F C_{3}-\left(C_{1}+C_{2}\right)^{2} \ll F
$$

The proof of equation (4.69) will justify the elimination of all $F C_{3}-\left(C_{1}+C_{2}\right)^{2}$ terms in equations (4.67) and (4.68) since in all these expressions there is a predominant $F$ term.

\section{Proof of (4.69)}

First from (4.12) we can easily see that:

$$
\begin{aligned}
& F(n)=\lambda F(n-1)+2 n T \overline{\hat{\omega}_{0, n} \cdot \bar{\omega}_{1, n}}+\overline{\hat{\omega}_{0, n}^{2}}+T^{2} n^{2} \overline{\hat{\omega}_{1, n}^{2}} \\
& C_{1}(n)=\lambda C_{1}(n-1)+T \overline{\hat{\omega}_{0, n} \cdot \hat{\omega}_{1, n}} \\
& C_{2}(n)=\lambda C_{2}(n-1)+T^{2} \overline{\hat{\omega}_{1, n}^{2}} \\
& C_{3}(n)=\lambda C_{3}(n-1)+T^{2} \overline{\hat{\omega}_{1, n}^{2}} .
\end{aligned}
$$

It can be easily seen as well using some simple algebraic manipulations that equation (4.69) is true for $n=1$ and $n=2$. Assuming that this is true for $n-1$ we will prove by induction that equation (4.69) is true for every $n$ : (We omit " and - on $\omega_{0}, \omega_{1}$ for simplicity of representation) 


$$
\begin{aligned}
& F(n) C_{3}(n)=\left[\lambda F(n-1)+2 n T \omega_{0, n} \omega_{1, n}+\omega_{0, n}^{2}+T^{2} n^{2} \omega_{1, n}^{2}\right] \cdot \\
& \text { - }\left[\lambda C_{3}(n-1)+T^{2} \omega_{1, n}^{2}\right] \\
& =\lambda^{2} F(n-1) C_{3}(n-1)+\quad \leftarrow A_{1} \\
& +\lambda F(n-1) T^{2} \omega_{1, n}^{2}+\left(2 n T \omega_{0, n} \omega_{1, n}+\omega_{0, n}^{2}+T^{2} n^{2} \omega_{1, n}^{2}\right) \\
& \text { - } \lambda C_{3}(n-\bar{I})+ \\
& \leftarrow B_{1} \\
& +T^{2} \omega_{i, n}^{2}\left(2 n T \omega_{0, n} \omega_{1, n}+\omega_{0, n}^{2}+T^{2} n^{2} \omega_{1, n}^{2}\right) \\
& \leftarrow C_{1} \\
& \left(C_{1}(n)+C_{2}(n)\right)^{2}=\left[\lambda C_{1}(n-1)+T \omega_{0, n} \omega_{1, n}+T^{2} n \omega_{1, n}^{2}+\lambda C_{2}(n-1)\right]^{2} \\
& =\lambda^{2}\left[C_{1}(n-1)+C_{2}(n-1)\right]^{2}+ \\
& \leftarrow A_{2} \\
& +T^{3} \omega_{0, n}^{2} \omega_{1, n}^{2}+T^{4} n^{2} \omega_{1, n}^{4}+2 T^{3} n \omega_{0, n} \omega_{1, n}^{3} \\
& \leftarrow C_{2} \\
& +2\left(T \omega_{0 n} \omega_{1 n}+T^{2} n \omega_{1, n}^{2}\right) \cdot \lambda\left[C_{1}(n-1)+C_{2}(n-1)\right] \leftarrow B_{2}
\end{aligned}
$$

Observing equations (4.71) and (4.72) one can see that $A_{1}-A_{2} \ll F$ because of the assumption. It is also obvious that $C_{1}-C_{2}=0 \ll F$. Hence we just have to prove that $B_{1}-B_{2} \ll F$ or:

$$
\begin{aligned}
\lambda F(n-1) T^{2} \omega_{1, n}^{2}+\lambda C_{3}(n-1) & \cdot\left(2 n T \omega_{0, n} \omega_{1, n}+\omega_{0, n}^{2}+T^{2} n^{2} \omega_{1, n}^{2}\right) \\
& -2 \lambda\left[C_{1}(n-1)+C_{2}(n-1)\right]
\end{aligned}
$$


- $\left(T \omega_{0, n} \omega_{1, n}+T^{2} n \omega_{1, n}^{2}\right) \ll F$.

Le us group the terms of the LHS of (4.73):

$$
\begin{array}{rlrl}
L H S & =[_{\sum_{k=0}^{n-1} \lambda^{n-k} \omega_{0 k}^{2}}+\underbrace{2 T \sum_{k=0}^{n-1} \lambda^{n-k} k \omega_{0 k} \omega_{1 k}}_{F_{1}}+\underbrace{T^{2} \sum_{k=0}^{n-1} \lambda^{n-k} k^{2} \omega_{1, k}^{2}}_{F_{3}}] T^{2} \omega_{1 n}^{2} \\
& +C_{3} \cdot\left(2 n T \omega_{0 n} \omega_{1 n}+\omega_{0 n}^{2}+T^{2} n^{2} \omega_{1 n}^{2}\right) & \\
& -2\left(C_{1}+C_{2}\right) \cdot\left(T \omega_{0 n} \omega_{1 n}+T^{2} n \omega_{13}^{2}\right) & \\
& =T^{2} \omega_{1 n}^{2} F_{3}-T^{2} n \omega_{1 n}^{2} C_{2} & \leftarrow A_{31} \\
& +T^{2} n^{2} \omega_{1 n}^{2} C_{3}-T^{2} n \omega_{1 n}^{2} C_{2} & \leftarrow A_{32} \\
& +T^{2} \omega_{1 n}^{2} F_{2}-2 T^{2} n \omega_{1 n}^{2} C_{1} & \leftarrow B_{31} \\
& +2 n T \omega_{0 n} \cdot \omega_{1 n} C_{3}-2 T \omega_{0 n} \omega_{1 n} \cdot C_{2} & \leftarrow B_{32} \\
& +T^{2} \omega_{1 n}^{2} \cdot F_{1}-T \omega_{0 n} \omega_{1 n} C_{1} & \leftarrow C_{31} \\
& +\omega_{0 n}^{2} C_{3}-T \omega_{0 n} \cdot \omega_{1 n} C_{1} . & \leftarrow C_{32}
\end{array}
$$

Now it can be seen that:

$$
\begin{aligned}
A_{31} & =T^{4} \omega_{1 n}^{2} \Sigma \lambda^{n-k} k^{2} \omega_{1 k}^{2}-T^{4} n \omega_{1 n}^{2} \Sigma \lambda^{n-k} k \omega_{1 k}^{2} \\
& =T^{4} \omega_{1 n}^{2} \Sigma \lambda^{n-k} \omega_{1 k}^{2} k(k-n),
\end{aligned}
$$




$$
\begin{array}{rl}
A_{32}= & T^{4} \omega_{1 n}^{2} \Sigma \lambda^{n-k} n^{2} \omega_{1 k}^{2}-T^{4} \omega_{1 n}^{2} \Sigma \lambda^{n-k} n k \omega_{1 k}^{2} \\
& =T^{4} \omega_{1 n}^{2} \Sigma \lambda^{n-k} n(n-k) \omega_{1 k}^{2}, \\
& A_{31}+A_{32}=T^{4} \omega_{1 n}^{2} \Sigma \lambda^{n-k} \omega_{1 k}^{2}(n-k)^{2} \\
B_{31} & 2 T^{3} \omega_{1 n}^{2} \Sigma \lambda^{n-k} k \omega_{0 k} \omega_{1 k}-2 T^{3} \omega_{1 n}^{2} \Sigma \lambda^{n-k} n \omega_{0 k} \omega_{1 k} \\
= & 2 T^{3} \omega_{1 n}^{2} \Sigma \lambda^{n-k}(k-n) \omega_{0 k} \omega_{1 k}, \\
B_{32} & =2 T^{3} \omega_{0 n} \omega_{1 n}\left(\Sigma \lambda^{n-k} n \omega_{1 k}^{2}-\Sigma \lambda^{n-k} k \omega_{1 k}^{2}\right) \\
& =T^{2}\left(\Sigma \lambda^{n-k} \omega_{1 n}^{2} \omega_{0 k}^{2}-\Sigma \lambda^{n-k} \omega_{0 n} \omega_{1 n} \omega_{0 k} \omega_{1 k}\right) \\
& =2 T^{3} \omega_{0 n} \omega_{1 n} \Sigma \lambda^{n-k}(n-k) \omega_{1 k}^{2}, \\
B_{31}+B_{32} & =2 T^{3} \Sigma \lambda^{n-k}(n-k) \cdot \omega_{1 k} \omega_{1 n}\left(\omega_{1 k} \omega_{0 n}-\omega_{1 n} \omega_{0 k}\right) \\
&
\end{array}
$$




$$
\begin{aligned}
C_{32} & =T^{2}\left(\Sigma \lambda^{n-k} \omega_{0 n}^{2} \omega_{1 k}^{2}-\Sigma \lambda^{n-k} \omega_{0 k} \omega_{1 k} \omega_{0 n} \omega_{1 n}\right) \\
& =T^{2} \Sigma \cdot \lambda^{n-k} \omega_{0 n} \omega_{1 k}\left(\omega_{0 n} \omega_{1 k}-\omega_{0 k} \omega_{1 n}\right) \\
& C_{31}+C_{32}=T^{2} \Sigma \lambda^{n-k}\left(\omega_{0 n} \omega_{1 k}-\omega_{0 k} \omega_{1 n}\right)^{2}
\end{aligned}
$$

Now assuming $T \omega_{1 n}<1$ we have:

$$
\begin{array}{ll}
\text { from (4.77): } & A_{31}+A_{32} \ll T^{2} \Sigma \lambda^{n-k} k^{2} \omega_{1 k}^{2}, \\
\text { from (4.80): } & B_{31}+B_{32} \ll 2 T \Sigma \lambda^{n-k} k \omega_{0 k} \omega_{1 k}, \\
\text { and from (4.83): } & C_{31}+C_{32} \ll \Sigma \lambda^{n-k} \omega_{0 k^{-}}^{2}
\end{array}
$$

Summing equations (4.84) we get:

$$
\begin{aligned}
L H S & \ll \Sigma \lambda^{n-k} \omega_{0 k}^{2}+2 T \Sigma \lambda^{n-k} k \omega_{0 k} \omega_{1 k}+T^{2} \Sigma \lambda^{n-k} k^{2} \omega_{1 k}^{2} \\
& =F
\end{aligned}
$$

which was io be proved. 
APPENDIX 4.4

Calculation OF THE SUR:S $S_{1}(n), S_{2}(n), S_{3}(n)$

(a)

$$
\begin{gathered}
S_{1}(n)=\sum_{s=0}^{n} \lambda^{n-s}=\lambda^{n}+\sum_{s=1}^{n} \lambda^{n-s} \\
\sum_{s=1}^{n} \lambda^{n-s}=\lambda^{n-1}+\lambda^{n-2}+\ldots+1=\sum_{s=0}^{n-1} \lambda^{*}
\end{gathered}
$$

Equations (4.85) and (4.86) give:

$$
\begin{aligned}
S_{1}(n) & =\lambda^{n}+\sum_{s=0}^{n-1} \lambda^{*}=\lambda^{n}+1+\sum_{*=1}^{n-1} \lambda^{*}=1+\sum_{s=1}^{n} \lambda^{*} \\
& =1+\frac{\lambda\left(1-\lambda^{n}\right)}{1-\lambda} \quad(\lambda \leq 1) .
\end{aligned}
$$

Hence

$$
S_{1}(n)=1+\frac{\lambda\left(1-\lambda^{n}\right)}{1-\lambda}
$$

(b)

$$
S_{2}(n)=\sum_{s=0}^{n} s \lambda^{n-1}=\lambda^{n-1}+2 \lambda^{n-2}+3 \lambda^{n-3}+\ldots
$$




$$
+(n-1) \lambda+n=\sum_{s=0}^{n-1}(n-s) \lambda^{S}=n \sum_{s=0}^{n-1} \lambda^{s}-\sum_{s=0}^{n-1} s \lambda^{s}
$$

But

$$
\sum_{n=0}^{n-1} s \lambda^{*}=\frac{\lambda-n \lambda^{n}+(n-1) \lambda^{n+1}}{(1-\lambda)^{2}}
$$

Equation (4.90) is obtained by differentiating equation (4.88). Hence:

$$
S_{2}(n)=n\left[1+\frac{\lambda\left(1-\lambda^{n-1}\right)}{1-\lambda}\right] \div \frac{\lambda-n \lambda^{n}+(n-1) \lambda^{n+1}}{(1-\lambda)^{2}}
$$

(c)

$$
\begin{aligned}
S_{3}(n) & =\sum_{s=0}^{n} s^{2} \lambda^{n-s}=1^{2} \lambda^{n-1}+2^{2} \lambda^{n-2}+\ldots \\
& +(n-1)^{2} \lambda+n^{2}=\sum_{s=0}^{n-1}(n-s)^{2} \lambda^{s}= \\
& =n^{2} \sum_{s=0}^{n-1} \lambda^{s}-2 n \sum_{s=0}^{n-1} s \lambda^{s}+\sum_{s=0}^{n-1} s^{2} \lambda^{s}
\end{aligned}
$$

In equation (4.92) only the last term need be calculated by differentiating equation (4.90) with respect to $\lambda$ :

$$
\sum_{s=0}^{n-1} s^{2} \lambda^{s}=\frac{\left[1-n^{2} \lambda^{n-1}+(n-1)(n+1) \lambda^{n}\right](1-\lambda)^{2}-(2 \lambda-2)\left[\lambda-n \lambda^{n}+(n-1) \lambda^{n+1}\right]}{(1-\lambda)^{4}}
$$

Hence finally equations (4.92) and (4.93) give: 


$$
\begin{aligned}
S_{3}(n) & =n^{2}\left[1+\frac{\lambda\left(1-\lambda^{n}\right)}{1-\lambda}-\lambda^{n}\right] \\
& -2 n \frac{\lambda-n \lambda^{n}+(n-1) \lambda^{n+1}}{(1-\lambda)^{2}} \\
& +\frac{\left[1-n^{2} \lambda^{n-1}+(n-1)(n+1) \lambda^{n}\right](1-\lambda)^{2}-(2 \lambda-2)\left[\lambda-n \lambda^{n}+(n-1) \lambda^{n+1}\right]}{(1-\lambda)^{4}}
\end{aligned}
$$

Recursive relationships are derived very easily:

$$
\begin{gathered}
S_{1}(n)=\lambda^{n}+\lambda^{n-1}+\ldots+1, \\
S_{1}(n+1)=\lambda^{n+1}+\lambda^{n}+\ldots+1
\end{gathered}
$$

Using equations (4.95) and (4.96) we obtain:

$$
S_{1}(n+1)=\lambda S_{1}(n)+1 .
$$

Similarly:

$$
\begin{aligned}
& S_{2}(n)=\lambda^{n-1}+2 \lambda^{n-2}+\ldots+(n-1) \lambda+n \\
& S_{2}(n+1)=\lambda^{n}+2 \lambda^{n-1}+\ldots+n \lambda+(n+1)
\end{aligned}
$$

Hence: 


$$
S_{2}(n+1)=\lambda \cdot S_{2}(n)+(n+1) \cdot
$$

Finally:

$$
S_{3}(n+1)=\lambda \cdot S_{3}(n)+(n+1)^{2}
$$




\section{Chapter 5}

\section{SYSTEM IMPLEMENTATION AND MEASUREMENTS}

\subsection{INTRODUCTION}

The main purpose of this chapter is to build a MCMH synthesizer and use it as a testing vehicle to study mainly TD short-term frequency stability (off-line prócessing) as well as adaptive frequency drift cancellation using a simple LMS algorithrs (on-line processing). Taking into account the approach to designing such a synthesizer, as presented in Section 3.3, we can consider that there are four major units which constitute the whole system: the VCO, the DAC subsystem, the digital counter. and the microprocessor-controller.

A similar system has been designed and tested in the past [Barsalou 82] but there are some basic differences to the one implemented here. The system built in [Barsalou 82] was meant to test the viability of the concept of this type of frequency synthesizer, whereas the system described in this chapter constitutes a complete MCMH synthesizer with enhanced capabilities of off-line frequency stability characterization. Its main function is to bop according to a predetermined frequency hopping pattern while at the same time achieving offset cancellation through on-line 
processing. Hence short-term VCO instability is being studied (Chapter 2) as well as the application of adaptive algorithms to correct for frequency departures due to temperature and VCO tuning voltage variations (Chapter 4). For this purpose, an INTEL 386 microprocessor together with the mathematics coprocessor (387) is used.

In Sections 5.2 to 5.5, the four major units, as mentioned in the '-eginning of this chapter, will be presented in some detail. It should be noted here, that the design follows the guidelines about the system requirements (as outlined in Section 3.4) which are meant to satisfy the specifications given in Table 3.1.

In Section 5.6, the development of the multipurpose software will be presented. This software is used (a) for verification and measurement of the system main characteristics, (b) for testing the TD short-term stability of the VCO, and (c) for adaptive cancellatión of systematic frequency drift: $\varepsilon$.

Finally, in Section 5.7, the operation and performance of the overall system will be presented. In the subsequent subsections, the behaviour of the source under various hopping patterns will be studied. In addition, the real-time adaptive frequency drift cancellation will be demonstrated.

\subsection{THE VOLTAGE-CONTROLLED OSCILLA- TOR}

It seems that several VCOs manufactured by Avantek [A vantek 84], Watkins Johnson [Watkins Johnson 82], and EMF [Barsalou 82] satisfy the primary requirements of tunable bandwidth (over $500 \mathrm{MHz}$ ) and tuning speed (settle to $0.01 \%$ accuracy within less than 100 nanoseconds for a full tuning voltage step). Specifically here, the Avantek VTO 8090 and the Watkins Johnson's V801 were the VCOs used throughout this work [Avantek 84], [Watkins Johnson 82]. Manufacturer's specifi- 
cations for these devices are also given in Appendix 5.1. However since it is difficult to produce a voltage step higher than $15 \mathrm{~V}$ to an accuracy of $0.01 \%$ in less than $1 \mu \mathrm{sec}$, only VCOs with a full voltage step less than 15 volts and producing more than $500 \mathrm{MHz}$ bandwidth are considered practical.

It should be mentioned here that hyperabrupt-junction-varactor VCOs produce a quite linear tuning curve in contrast to the abrupt junction models [Watkins Johnson 80 ] because the frequency-voltage relationship is given by the following equation:

$$
f=K \cdot V
$$

where $K$ is a constant.

On the other hand, if more bandwidth is needed for the same full voltage step we should expect to afford a higher modulation sensitivity (MS). Below, a definition is given for the "optimum" modulation sensitivity $M S_{0}$ :

$$
M S_{0}(M H z / V) \triangleq \frac{\text { Tunable Bandwidth (MHz) }}{\text { Full Voltage Step (V) }}
$$

The term "optimum" is added because it is well known that existing devices do not exhibit a uniform modulation sensitivity throughout their tunable bandwidth. The result of this is that there are regions in the tuning curve where the modulation sensitivity is somewhat higher than $M S_{0}$. Varactor-tuned VCOs exhibit an overall change in MS which is due to a decrease in the rate of change of the varactor capacitance as a function of voltage. By increasing MS in order to increase the tunable bandwidth of the VCO, the noise immunity of the system is degraded.

- Hence there should be a trade-off between higher noise and higher bandwidth. ,

There are several VCOs satisfying the $15 \mathrm{~V}$ full step requirement with $500 \mathrm{MHz}$ 
bandwidth. Several of these are shown in Appendix 5.1.

Now some aspects of the noise behaviour of wide frequency tuning sources will be examined and their differences to very limited tuning (up to 1\%) frequency sources will be pointed out.

In the technical literature sbout VCOs ([Watkins Johnson 80], [Frequency Sources 79], [Bisseger 77]) FM noise is the name given to random fluctuations of a VCO's output frequency. However in the theory of frequency stability, these fluctuations are specified as short term frequency stability and can be measured with counters as we have explained in detail in Chapter 2. It is also of importance to note, [Frequency Sources 79], that the VCO frequency noise is usually due to:

(a) thermal noise exhibiting a flat power spectral density ([Papoulis 65]),

(i) low frequency (flicker) "1/f noise".

All the important differences in noise behaviour between VCOs and frequency sources which have very limited tuning or are free running, derive from the fact that broadband VCOs are low-Q devices, mainly due to varactor losses. Because of the low $Q$ and high MS, even peripheral elements such as RF chokes ard bias filters can have an effect on frequency drift. This is how frequency pushing is created [Chalmers 79]. On the other hand, output frequency is easily pulled by external mismatches for the same reason. It is of importance to note that hyperabruptvaractor VCOs have a lower $\mathbf{Q}$ than the equivalent abrupt models making them more susceptible to all the above noise sources.

Another phenomenon, called short-term post tuning drift (PTD), is mainly caused by changes in the chip temperature of the varactor diode brought about by changes in the bias and RF power dissipation following a frequency step [Buswell 75]. Varactor charging which represents a build-up of impurity jons around the junction 
is the main cause of long-term PTD. The charging effect shanges the capacitance of the varactor resulting in this kind of disturbance.

\subsection{THE DIGITAL-TO-ANALOG CONVERSION SUBSYSTEM}

As was shown in Section 3.3 we need a 16-bit DAC tuning in full voltage step (10 to 15 volts) within $1 \mu \mathrm{sec}$ with an accuracy of $0.01 \%$ of the final value. Although the output of $\mathrm{D} / \mathrm{A}$ converters is often in the form of voltage, most manufacturers provide an output current instead of a voltage in their DACs. This happens because, due to the basic conversion process, a current output is quite fast, linear and free from offset [Analog Devices 80]. Present state-of-the-art indicates that there are no on-chip IC op-amps with submicrosecond settling time to useful resolution [Analog Devices 80]. The inevitable consequence expressed by design tradeoffs is that the op-amp will increase the settling time, limiting the performance of the DAC. However the speed of response can be controlled by the user if an external op-amp is connected to the current output of the DAC. It also depends on the user to choose between the inverting and the noninverting mode.

The DAC subsystem used here has the general form shown in Figure 5.1, whereas a detailed schematic of this system as built and used in this thesis is illustrated in Appendix 5.2. There are some 16-bit DACs reaching an accuracy of $0.01 \%$ with 1 microsecond [Harris 84]. There are also several video op-amps ([Burr-Brown 86], [Analog Devices 82]) settling to $0.01 \%$ in 1 microsecond. Hence

we can see that since VCOs settle within the required accuracy in several tens of nanoseconds, the major con! ributor to settling time is the DAC subsystem as has also been noted in [Barsalou 82].

The rest of this section is devoted to a brief explanation of the relationship 


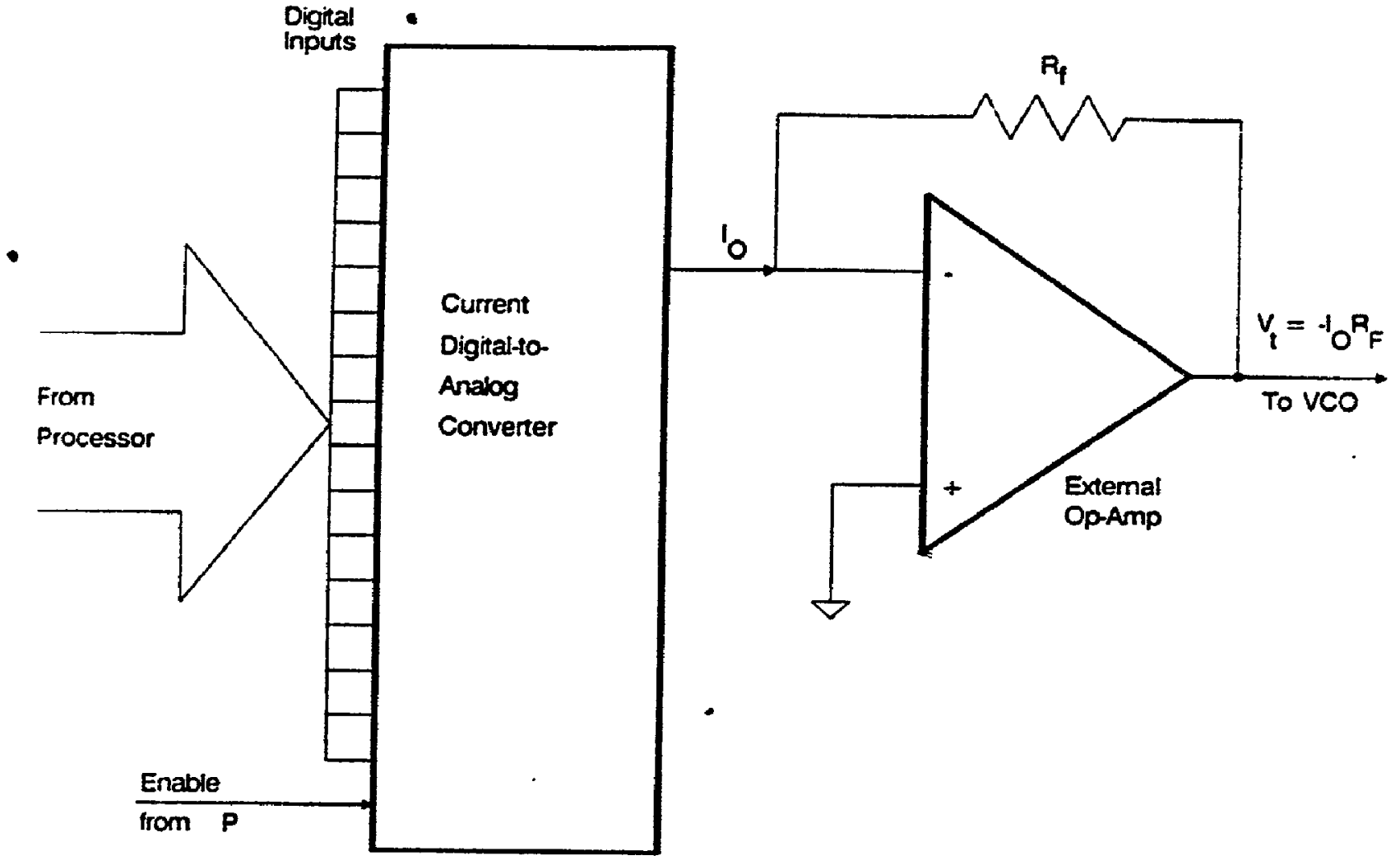

Figure 5.1: DAC subsystem 
between the settling time and both closed- and open-loop circuit parameters, the main reason being that settling time is the most critical factor imposing limits to the speed of the synthesizer and yet is sometimes misunderstood by systems designers.

First of all, the step response of an ideally linear $6 \mathrm{~dB} /$ octave amplifier with a closed-loop bandwidth (CLB) of $f_{0}$, as in equation (5.3) is shown in Figure 5.2(a). The CLB is

$$
f_{0}=\frac{1}{2 \pi R_{F} C_{\text {out }}}
$$

where $R_{F}$ is the feedback resistor (Figure 5.1) and $C_{\text {out }}$ is the output capacitance of the DAC.

Hence from Figure 5.1 and equation (5.3) one can see that by increasing $\boldsymbol{R}_{F}$ in order to get a higher output voltage $V_{t}$, the CLB decreases and from Figure 5.2(a) the response of the circuit becomes slower. However since the settling time of the op-amp is determined by both linear and nonlinear characteristics and because it is a closed loop parameter [Analog Devices 82] it cannot be calculated from the open-loop specifications such as slew rate, small signal bandwidth, etc. This can be readily seen in Figure 5.2(b) which shows that although there is a relationship between slew rate $S$ and settling time, this relationship is not linear. Despite this fact, some authors ([Schoniger 77]) provide empirical equations relating $S$ to the settling time via the rise time $t_{r}$ :

$$
S=\frac{\Delta V_{t}}{t_{r}}
$$

where

$$
t_{\boldsymbol{r}}=\mathbf{2 . 2 \tau}
$$



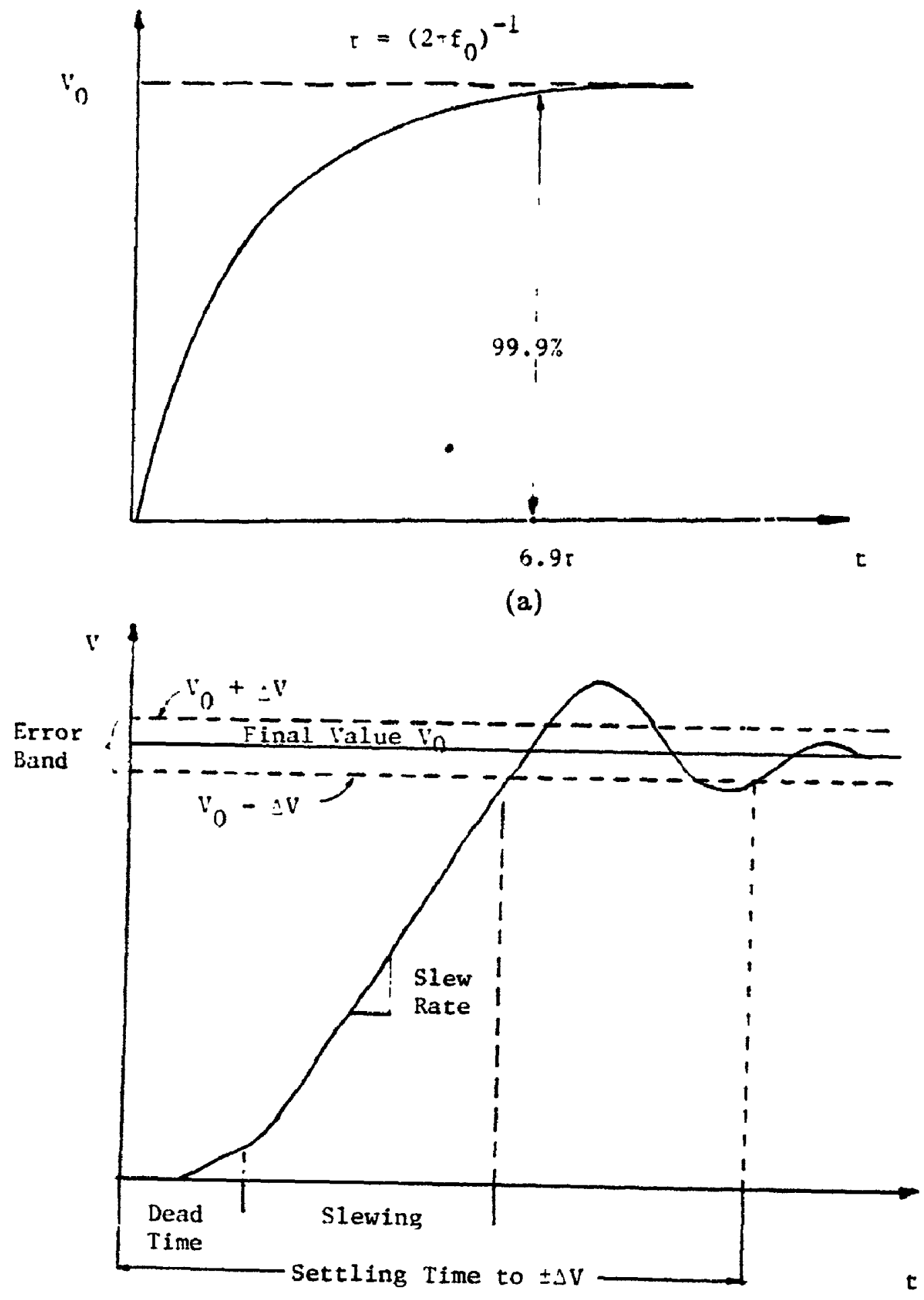

(b) Figure 5.2: (a): Step response for linear $6 \mathrm{~dB} /$ octave amplifier; (b): typical settling
time characteristics 
and

$$
\tau=\left(2 \pi f_{0}\right)^{-1}
$$

This rise time corresponds to the output response to a step input betwen the $10 \%$ and $90 \%$ points. According to the same author, the time required for the voltage $V$ to reach its final value $V_{t}$ within $0.01 \%$ is $9.2 \tau$ because the following equation is true:

$$
V=V_{t}\left(1-e^{-t / \tau}\right)
$$

Although these equations can help the designer to determine a region for the slew rate of the needed op-amp, it is anticipated that since the settling time is a nonlinear function (i.e., it depends on the input signal level and can be affected by external impedances of the amplifier), there is no way of predicting the settling time value from the above equations. Therefore the only guarantee of its value is the explicit specification of the op-amp manufacturer.

There are some video op-amps [Burr-Brown 86], [Analcg Devices 82] with submicrosecond settling times within $0.01 \%$ which exhibit slew rates of 100 to 200 $\mathrm{V} / \mu \mathrm{sec}$, which is considered reasonable for the application at hand.

\subsection{THE COUNTER}

As was stated in Chapters 2 and 3, the counter constitutes a major subsystem for the MCMH synthesizer since it is used for both the TD stability characterizaiion of the hopping VCO and it is the feedback path for the clo: $d$ loop system such that its output is used for the correction of the frequency offsets. 
The counting is performed by down-converting the output of the VCO through a stable local oscillator to a frequency range $(0-250 \mathrm{MHz})$ which can be directly handled by the counter.

In Appendix 5.3 one can see the specific circuit that was used, with the ECL and TTL parts as indicated. For an accuracy of approximately $15 \mathrm{KHz}$ a count period of $(15 \mathrm{KHz})^{-1}$ was found adequate.

Since it is not the purpose of this thesis to examine in detail how a high frequency counter is designed, the interested reader is referred to [Barsalou 82] and [Bloor 80]. However some general aspects of this design will be briefly addressed. In the first stage of the counter, ECL components should be used because there are no TTL counters going up to $300 \mathrm{MHz}$. However Motorola MC1654 4-bit counter is suitable for the fi-t stage since it goes up to $300 \mathrm{MHz}$. The subsequent stages of the counter are TTL since the highest ripple-carry-out from MC1654 will be less than $16 \mathrm{MHz}$. The counters used for the low frequency portion are 74161 synchronous counters [Texas Instruments 81]. Three of these chips are needed since for a maximum intermediate frequency (IF) of $250 \mathrm{MHz}$ and resolution of $10 \mathrm{KHz}$, the maximum count could be 25,000 .

In the ECL part of the counter there are three other types of ICs:

1. A comparator,

2. The ECL-to-TTL translators,

3. The TTL-to-ECL translators.

The comparator (MC1651) converts the analog IF to a digital square waveform. This waveform is then counted by the counter, the output of which is connected to the ECL-to-TTL translators. These are useful in order to make this portion of the 
counter TTL compatible. The third type of ECL ICs used here is the TTL-to-ECL translators which are needed to control the ECL part of the counter (enable-reset etc.), using TTL outputs coming from the microprocessor ( $\mu \mathrm{P})$. Because of the fast rise time of the 1600-series components, the ECL part of the counter needs a large ground plane and good impedance matching by using microstrip transmission line sections and suitable terminations. All these considerations indicate that the ECL part of the counter should be installed on a separate PC board, while the TTL portion may be wire-wrapped on another board which can also contain the LS373 latches used to latch the count into the microprocessor through an Intel 8255 Programmable Peripheral Interface (PPI). 100 mil wide lines must be used on the 62 mil-thick substrate to match the output of the IF mixer to the input of the MC1651 comparator. This provides an impedance of $Z_{0}=50 \mathrm{Ohm}$. After the comparator, 60 mil wide lines are needed $\left(Z_{0} \simeq 68 \mathrm{Ohm}\right)$ to interface the remaining $E C L$ chips. The parallel termination scheme is used, which refers to a line terminated at the receiving end, through a resistor of value equal to the charactèristic line impedance $Z_{0,}$ to -2 volts. In order to avoid this third voltage level of -2 volts, the other two being $+5 \mathrm{~V}$ and $-5.2 \mathrm{~V}$, the scheme indicated by a star $\left(^{*}\right)$ in Appendix 5.3 can be used according to which $V_{E E}\left(V_{E E}=-5.2 \mathrm{~V}\right)$ is applied via two resistors $R_{1}$ and $\boldsymbol{R}_{2}$. The parallel terminating scheme is preferred to the series scheme because it can drive distributed loads. The open-line termination is not suitable because there are restrictions imposing maximum line lengths. Finally it has to be noted that the ground plane need not cover $100 \%$ of the PC board surface but $30-40 \%$ can be removed for signal interconnections. 

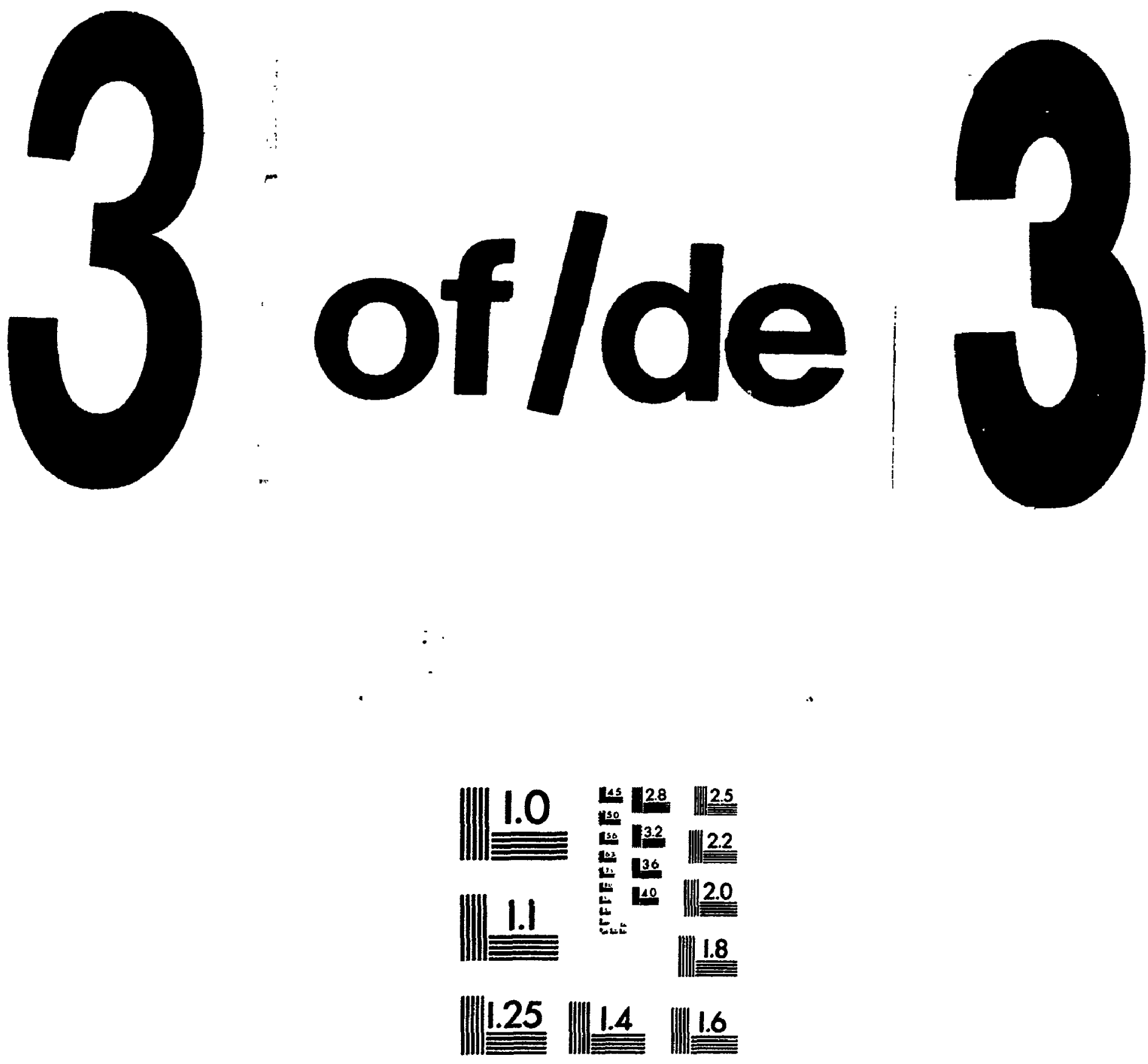

MICROCOPY ATE'SLUUTION TEST CHART

NATIONAL E:IPEAL' OF STANDARDS

STANDARD REFEKENCE MATERIAL 1010a

(ANSI and ISO TEST CHART NO 2) 
counter TTL compatible. The third type of ECL ICs used here is the TTL-to-ECL translators which are needed to contsol the ECL part of the counter (enable-reset etc.), using TTL outputs coming from the microprocessor $(\mu \mathrm{P})$. Because of the fast rise time of the 1600-series components, the ECL part of the cotnter needs a large ground plane and good impedance matching by using microstrip transmission line sections and suitable terminations. All these considerations indicate that the ECL part of the counter should be installed on a separate PC board, while the TTL portion may be wire-wrapped on another board which can also contain the LS373 latches used to latch the count into the microprocessor through an Intel 8255 Programmable Peripheral Interface (PPI). 100 mil wide lines must be used on the 62mil-thick substrate to match the output of the IF mixer to the input of the MC1651 comparator. This provides an impedance of $Z_{0}=50 \mathrm{Ohm}$. After the comparator, 60 mil wide lines are needed $\left(Z_{0} \simeq 68 \mathrm{Ohm}\right)$ to interface the remaining ECL chips. The parallel termination scheme is used, which refers to a line terminated at the receiving end, through a resistor of value equal to the characteristic line impedance $Z_{0}$, to -2 volts. In order to avoid this third voltage level of -2 volts, the other two being $+5 \mathrm{~V}$ and $-5.2 \mathrm{~V}$, the scheme indicated by a star $\left(^{*}\right)$ in Appendix 5.3 can be used according to which $V_{E E}\left(V_{E E}=-5.2 \mathrm{~V}\right)$ is applied via two resistors $R_{1}$ and $\boldsymbol{R}_{2}$. The parallel terminating scheme is preferred to the series scheme because it can drive distributed loads. The open-line termination is not suitable because there are restrictions imposing maximum line lengths. Finally it has to be noted that the ground plane need not cover $100 \%$ of the PC board surface but $30-40 \%$ can be removed for signal interconnections. 


\subsection{THE MICROPROCESSOR AND ITS INTER- FACE TO THE REST OF THE SYSTEM}

In this synthesizer the microprocessor $(\mu \mathrm{P})$ constitutes both the controlling and the processing element. Its main controlling functions are the following:

(a) To provide the digital word to the DAC at the appropriate time.

(b) To start the counter wi.en steady state conditiuns have been established at the VCO output.

(c) To stop the counter and retrieve the count through an input port.

(d) To clear the counter and start all over again.

This procedure should be repeated every $100 \mu \mathrm{sec}$ approximately. The main processing function of the $\mu \mathrm{P}$ is to use the last three samples and a simple adaptive algorithm in order to predict the expected excursion of the output frequency from its nominal value such that the $\mu \mathrm{P}$ can adjust the $\mathrm{DAC}$ words to prevent possible drifting in frequency of the output of the system. All these calculations and adjustments should be done within the time period during which the output frequency is counted ( $\sim 100 \mu \mathrm{sec})$. Finally, the system must have the capability of permanently storing several hundred Kbytes of digital words. This is mainly needed for off-line TD frequency stability characterization of the hopping VCO. Part of that storage space is needed to store a pseudorandom sequence that dictates the hopping pattern. A PC system built around the Intel $386 \mu \mathrm{P}$ was found to have adequate processing power and storage capabilities for our purpose. Of course, the $\mu \mathrm{P}$ has to interface 16-bit data with the counter, plus a 16-bit digital word applied to the DAC, plus 4-bits assigned for controlling the whole system. Hence there is a need for one 16-bit input port to retrieve the 16-bit count into the $\mu \mathrm{P}$, one 16-bit output 
port to issue the 16-bit digital word to the DAC and a 4-bit output port for control. This is done by using 2 Intel 8255 Programmable Peripheral Interface (PPI) chips. A descriptive schematic of the interface is shown in Figure 5.3, while the actual I/O interface used is shown in detail in Appendix 5.4. This $1 / O$ design was arrived at by expanding an idea in [Drummond 87]. Each of the two PPIs have 8-bit ports (A, B and C). The values that are set in the control register of the 8255 determine which of the three ports are inputs and which are outputs. But one 8255 cannot be used for word addressing ([Drummond 87]). However, two 8255 can be used with one chip corresponding to the most significant byte and the other corresponding to the least significant byte of the address. The chips are used in Mode 0 and control word \#2 is employed to make ports $A$ and ports $C$ outputs while ports $B$ are inputs ([Intel 82]). Since two 8255s are used, additional address decoding is needed using a 74LS138 decoder (see App.5.4). This chip uses as inputs $A_{0}, A_{3}$ and $A_{4}$ of the PC address bus to select the first or second 8255 by connecting two of its outputs to the $\overline{C S}$ input of the PPIs. (see App.5.4). The 74LS682 is used to impose the location of the physical addresses of the two $I / O$ in the memory space. This is done by appropriately setting, using a DIP switch, the $A_{5}$ to $A_{9}$ bits of the PC address bus.

The four control bits are generated from port $C$ of the second PPI (8255B) as shown in Figure 5.3, and they were used:

(a) To reset the counter (CLEAR).

(b) To enable the flip-flop of LS273 in order to latch the 16-bit word into the DAC (LATCH DAC).

(c) To enable the counter ( $\overline{S T O P})$.

(d) To latch the data from the counter into the $\mu \mathrm{P}$ through two LS373 chips (LATCH COUNT). 


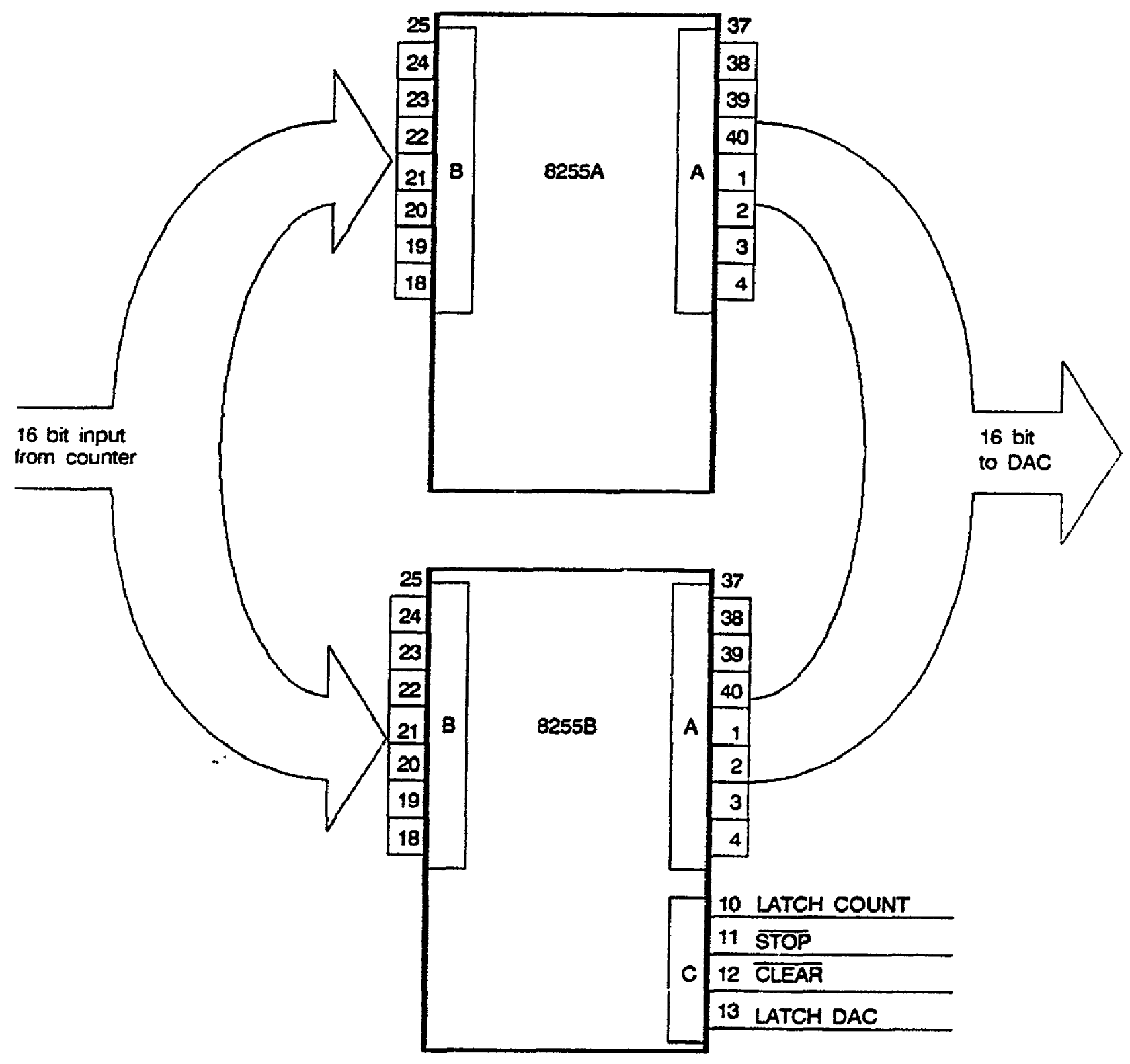

Figure 5.3: Schematic of $I / O$ interface with system 


\subsection{SOFTWARE DEVELOPMENT}

Figure 5.4 demonstrates the generation of the software and how it interacts with the system in order to produce either testing conditions for the system or actual hopping conditions. By the term "testing conditions" (Testing Hardware (TH) mode) we mean imposing an infinite hopping procedure (usually between two frequencies) which is useful to evaluate the performance of the hardware in terms of speed, timing and subsystem verification. (Counter, DAC etc.). During the actual Frequency Hopping (FH) mode, the system is commanded to operate either in the open loop mode (Time domain frequency stability evaluation) or in the closed loop (adaptive correction) mode. Results obtained by operating in TH or FH modes will be presented in detail in the next section. We now proceed to examine the software and its capabilities in more detail. To describe Figure 5.4 we refer to the numbers appearing therein:

1. The various hopping patterns are generated through a Fortran Controlling Program (FCP).

2. A specific hopping pattern is loaded to a memory space from which the Assembly System Commanding program (ASCP) can retrieve it through a suitable software interface between FCP and ASCP. The main instructions of the Fortran and Assembly programs creating this interface are shown in Figure 5.5.

3. Now the system executes ASCP through a batch file which links the object files of FCP and ASCP and produces an executable program to command and control the hardware (step 4.)

4. MCMH synthesizer is now operating either in the $\mathrm{TH}$ or the $\mathrm{FH}$ mode under the control of ASCP. 


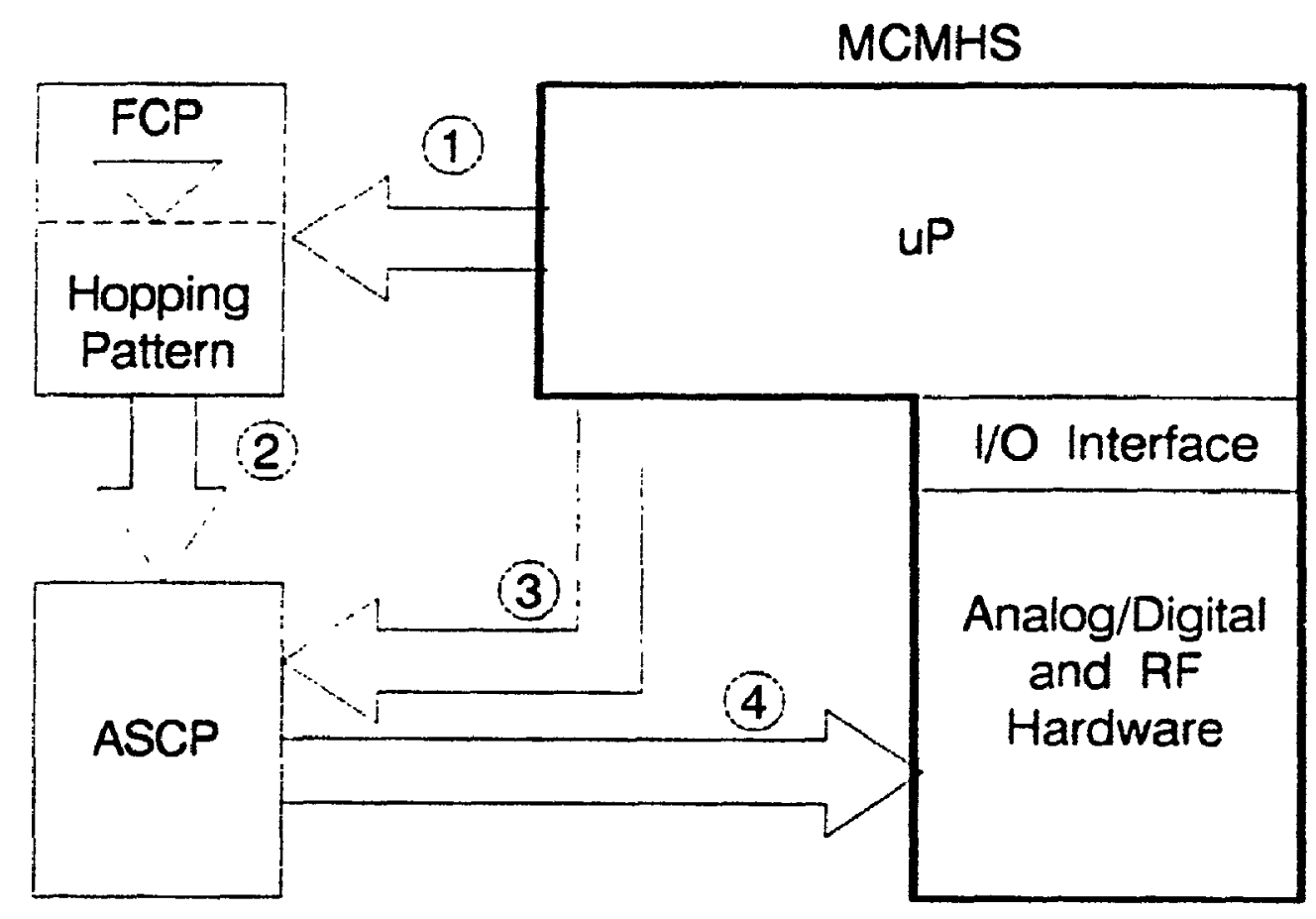

Figure 5.4: Software generation and interaction with the system 


\section{FCP}

common/bla/ rngl(16384), rng2(16384), res1(16384), res2(16384)

call adjhop (rng1,rng2)

\section{ASCP}

Public ADJHOP

LES BX,DWORD PTR[BP].8

SAR BX,1

SAR BX,1

SAR BX,1

SAR BX,1

MOV AX,ES

ADD AX,BX

MOV RNGSEG,AX

ADD AX,1000H

MOV ES,AX

$\vdots$

MOV DS,RNGSEG

MOV AL, $[\mathrm{BX}]$

FISTP WORD PTR ES:[DI]

Figure 5.5: Main instructions interfacing FCP to ASCP 
The way this is done is examined in detail in Figure 5.6. Here again, we proceed to describe in detail all the steps as shown in this figure by referring to their respective numbers:

1. The system is initialized by taking the following steps:

(a) In the port initialization step the two 8255 PPI ports $A$ and $C$ become outputs and ports $B$ inputs. This is done by suitable programming of the control words.

(b) In the DAC word-allocation and storage step 100 digital words representing 100 different points in the VCO tuning curve are placed in the data segment of the assembly program. These words represent frequencies on the $1.5 \mathrm{GHz}$ region located approximately $1 \mathrm{MHz}$ apart.

(c) The memory allocation for a random number array is done immediately after the data declaration. The DS register is used as the segment of address of the random number array with offset 0 . The occasional random number is an integer 1 to 100 which can be used as an index to address the DAC word table.

(d) The counter outputs or any other important variables that need to be stored are given a memory space using the ES register as the segment of their address with offset 0 .

(e) Finally initialization of the LMS adaptive algorithm is needed by assigning values for the adaptation coefficient $\mu$ as well as the initial signal and weight vectors.

2. The $\mu \mathrm{P}$ issues the command to apply a suitable $\mathrm{DAC}$ word which may have been adjusted during the previous iteration if the system is working in the closed-loop mode (see point 6.) or it may have been left unaffected for operation in the open-loop mode. 


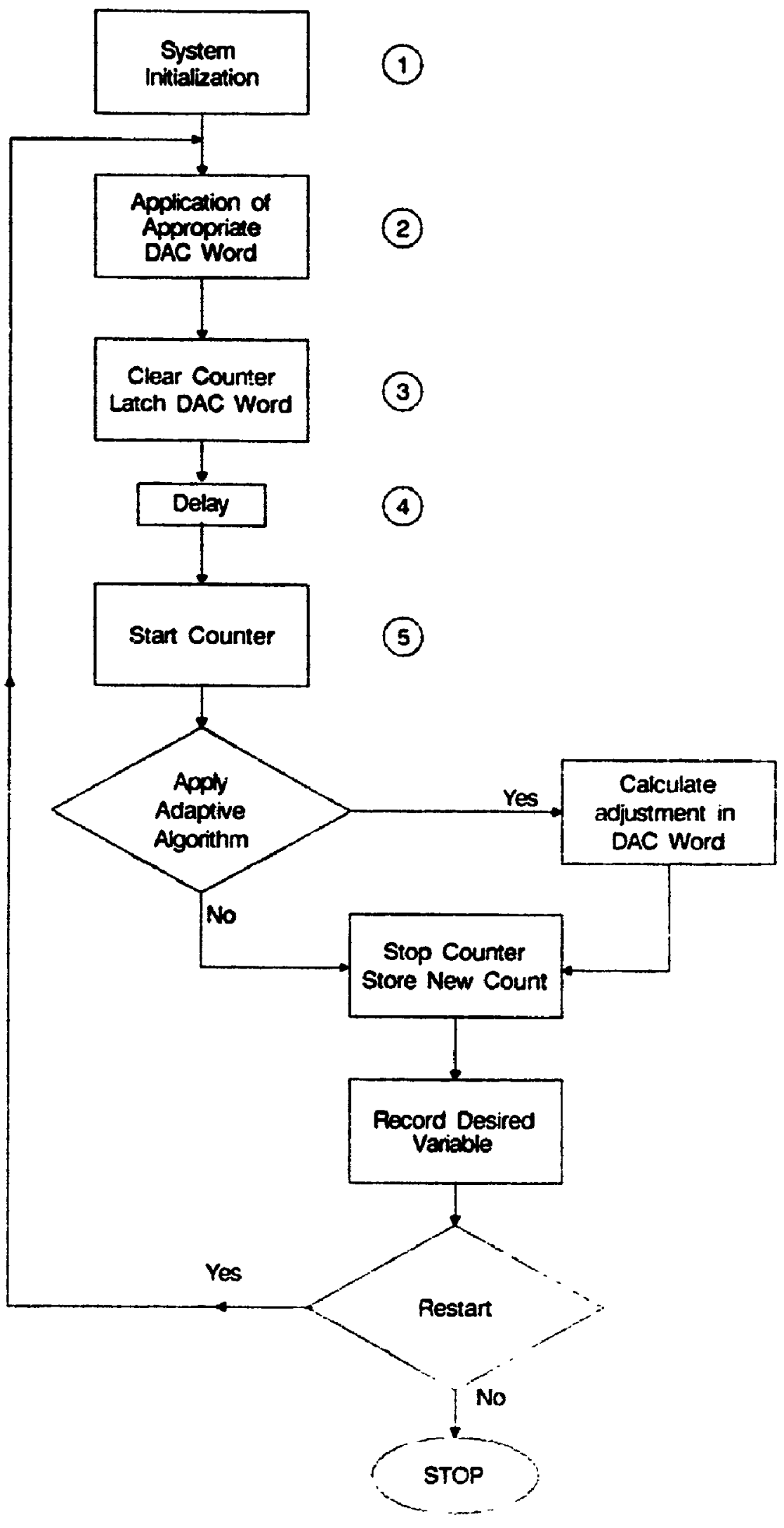

(6)

(7)

(8)

Figure 5.6: Assembly commanding program (ASCP) structure 
3. A control word is issued by the $\mu \mathrm{P}$ to clear the counter and latch the DAC word to the DAC through the two 74LS273 chips (App.5.2).

4. A small delay is added to allow steady state conditions to be established in both the VCO and the DAC subsystem. Now the system is operating at a particular frequency $f_{r}$.

5. After that, another control word is issued by the $\mu \mathrm{P}$ to start the counter.

6. While the counter is counting the down-converted version of $f_{r}$, the program performs calculations by using a simple LMS alyorithm to obtain a prediction of the value of the frequency currently counted. From the outcome of the algorithm an adjustment is applied to the DAC word.

All the above calculations are done only if the system operates in the closedloop mode. If not, we go immediately from 5 . to 7 .

7. After allowing the counter to count for approximately $125 \mu \mathrm{sec}$, a third control word is issued to stop the counter and store the outcome into the $\mu \mathrm{P}$ by using a pair of 74LS373 chips to interface the counter to the $\mu \mathrm{P}$ peripherals.

8. Finally we may need to store some variables such as the error between the predicted and the actual count, the new weight vector or any other variable that may be needed to test the system operation (i.e., we may need to verify that the system retrieves the random number array properly, and for this purpose we may store somewhere this variable and check it against the Fortran generated value to see if they match). 


\subsection{THE OVERALL SYSTEM: TESTING AND MEASUREMENTS}

\subsubsection{Hardware Verification}

The system is set to operate first in the TH mode by letting it hop between two frequencies in an infinite loop. In this way the following aspects can be verified.

(a) Counter Verification

This is done by assigning a "hopping pattern" of only one frequency and having the system operating indefinitely without stopping the counter such that we can connect the TTL outputs of the counter (MECL chip 10125) to a high frequency oscilloscope and obtain the counting waveforms seen in Figure 5.7. These were obtained using an IF input to the counter of $200 \mathrm{MHz}$. Figure 5.7(a) shows the LSB of the counter while Figure 5.7(d) represents the MSB. It should be noted that the voltage levels are 10 times higher than indicated in pictures ( $\times 10$ probe was used). The LSB is not a good square wave while there is some undershooting in all the waveforms possibly due to insufficient matching. The peak-to-peak voltage of the LSB is slightly lower than the rest. By decreasing the IF it was observed that the LSB becomes a good square wave.

(b) Adaptive Algorithm Timing

We let the system operate under the same conditions as in (a) with the exception that the counter is controlled as it would be under actual hopping conditions. We connect the scope probe on the control output which is maintained at a high logic level as long as the counter is counting. This is done while the $\mu \mathrm{P}$ performs the calculations and processing to obtain a predicted value for the count using the LMS algorithm. Figure 5.8(a) is a picture of this waveform where it is shown that 


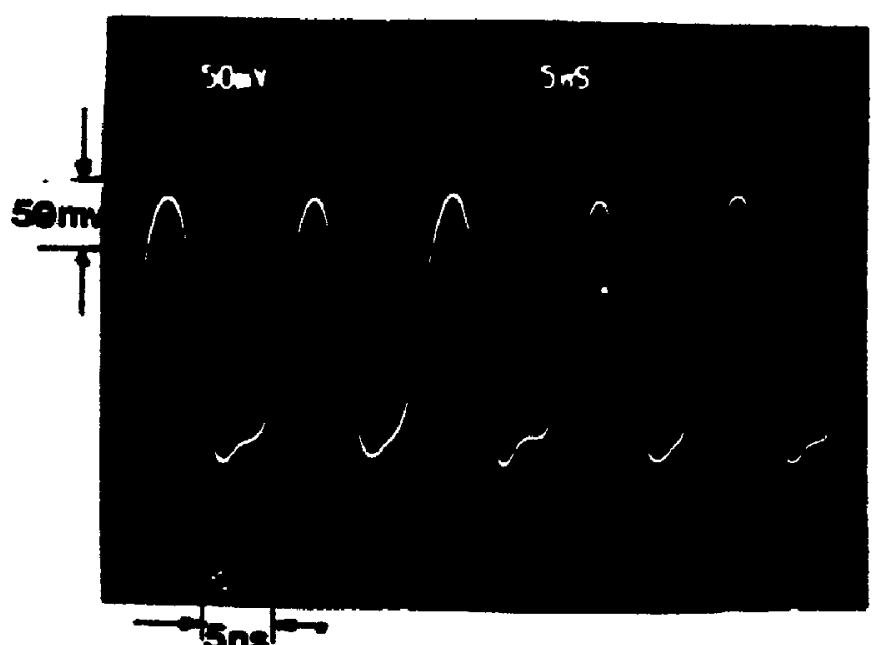

(a)

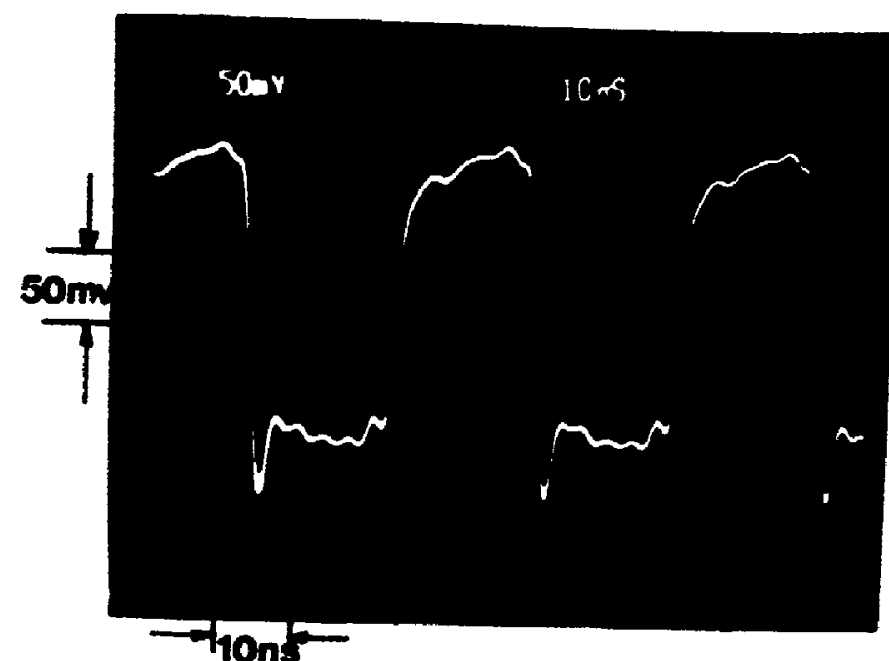

(c)

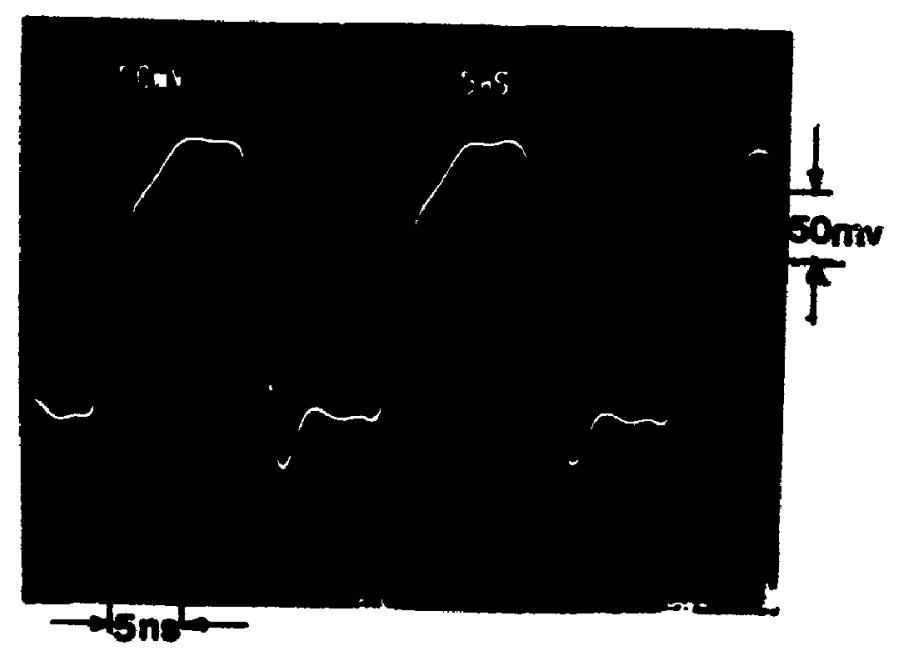

(b)

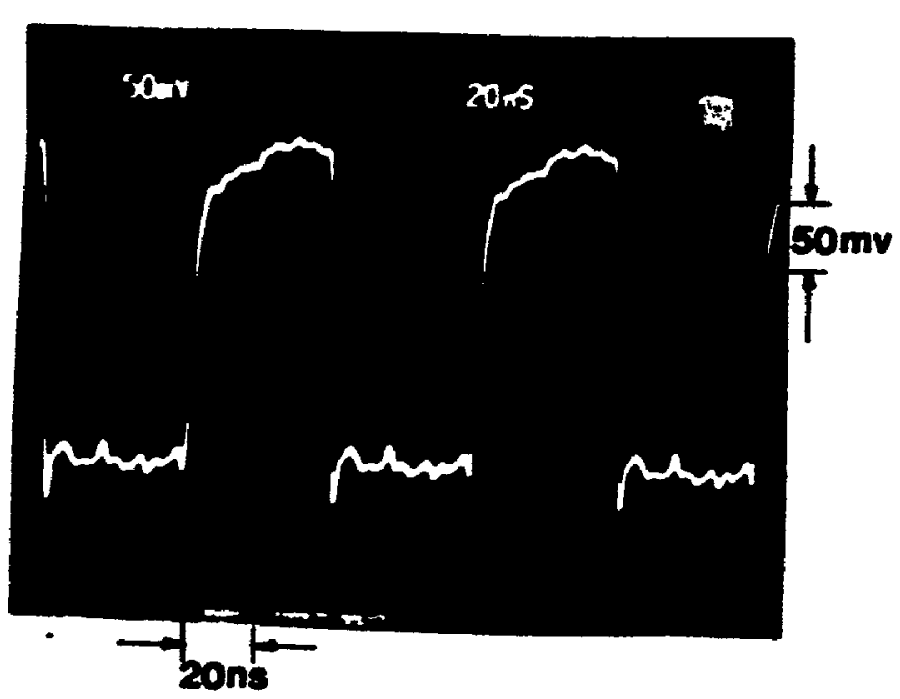

(d)

Figure 5.7: Counter output waveforms for an IF of $200 \mathrm{MHz}$ 


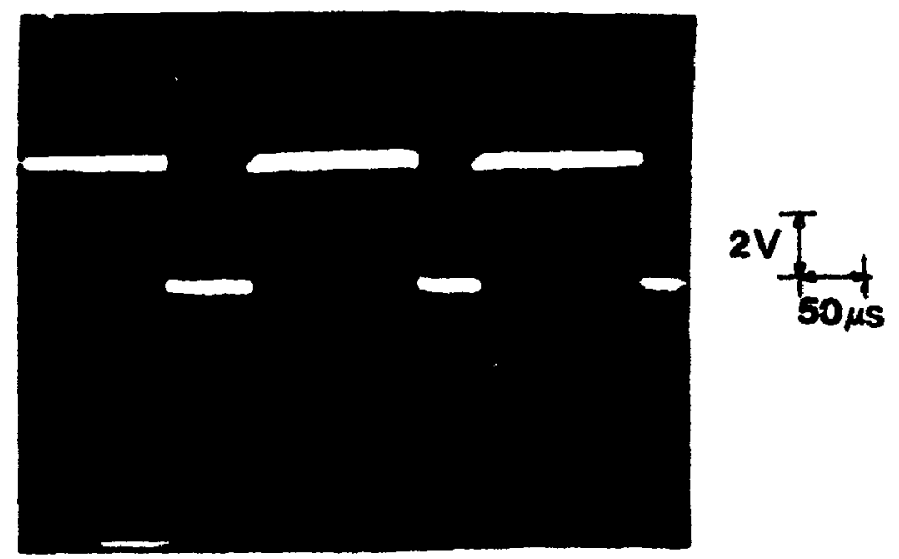

(a)

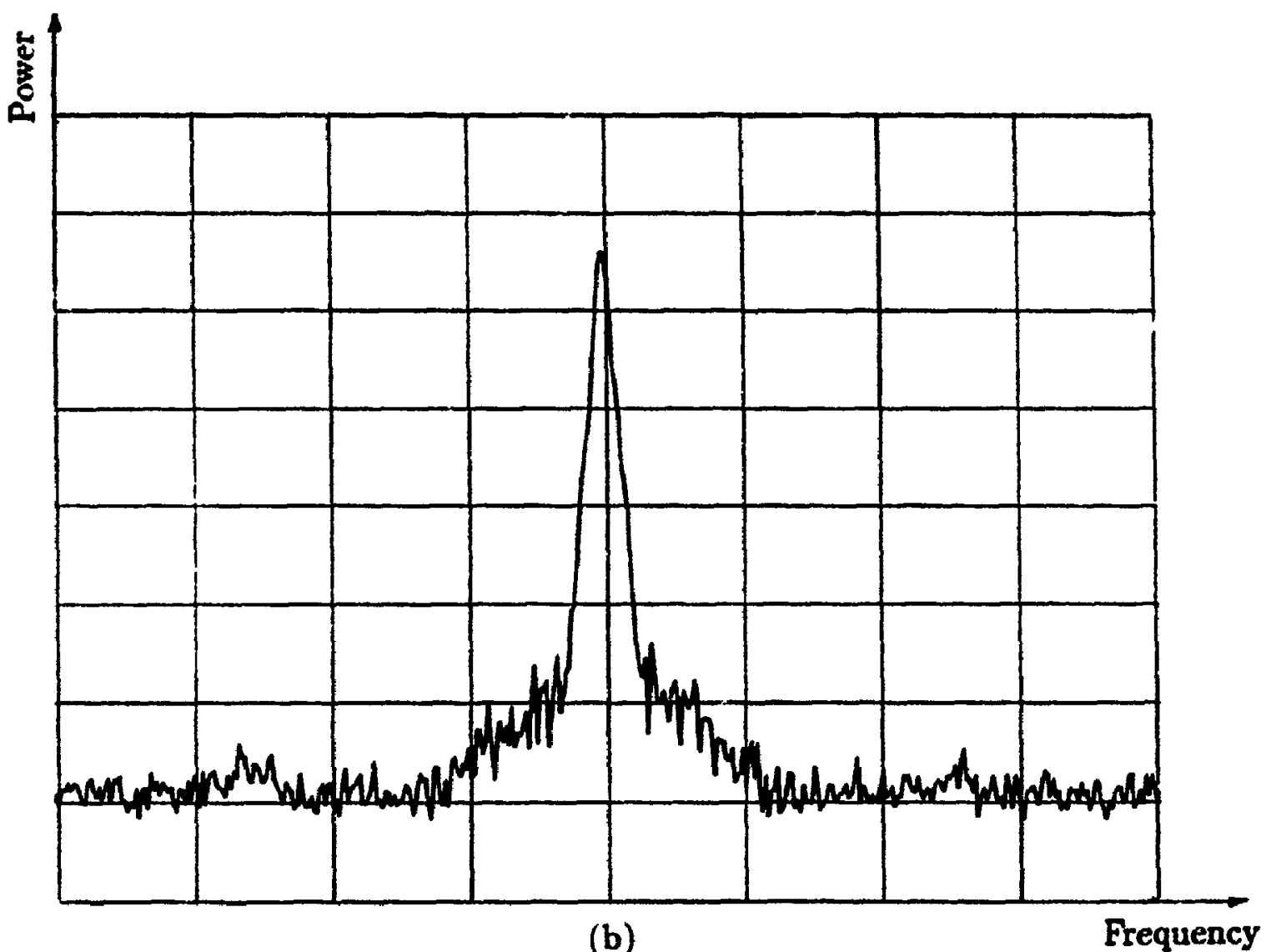

Figure 5.8: (a): Algorithm timing (high section of scope trace) (Horizontal: 50 $\mu \mathrm{sec} /$ div, Vertical: 2V/div; (b): VCO output while it continuously issues one frequency (CTR $=1.0239 \mathrm{GHz}$, Horizontal: $2 \mathrm{MHz} / \mathrm{div}$, Vertical: $10 \mathrm{~dB} / \mathrm{dir}, P_{\text {ref }}=18$ $\mathrm{dBm})$ 
the counter is gated for approximately $125 \mu$ sec.

(c) The VCO Output

The VCO was mounted on a PC board which was then put into a metal box. Figure 5.8(b) depicts the output spectrum of the VCO while the system is operating under the same conditions as in (b). A shielded coaxial cable connects the DAC output to the $V_{t}$ VCO input. Without a shielded cable connection the phase noise in the output spectrum is considerabiy higher.

(d) Transient Response

As discussed in Section 5.3, the elements limiting the speed of the system are the DAC and the video op-amp connected to the output of the DAC to convert the current output to a voltage output. The specification sheets for both the particular DAC and op-amp used here (see App.5.2) state that both settle within $0.01 \%$ of the final value in less than $1 \mu$ sec. An accurate determination of the transient response of this system to step inputs is quite difficult, but we can get a reasonable estimate of it by using the same measurement method as in [Barsalou 82]. A frequencyto-voltage converter (frequency discriminator) is used to transform frequency steps into the VCO output into voltage steps suitable for observation on a scope. The system is set again in an infinite hopping mode. However, this time there are two frequencies between which it is continuously switching. The input of the IF discriminator is connected at the IF output of the system (the one going to the counter) while its video output is connected to an oscilloscope. The RHG DT7020 Limiter/Discriminator (L/D) was used. It was found to exhibit an IF of $70 \mathrm{MHz}$ and linear bandwidth of approximately $30 \mathrm{MHz}$. Its response is the typical discriminator $S$ curve with positive and negative peaks at 85 and $50 \mathrm{MHz}$ respectively. There are nulls (zero voltage offset) at $35,45,70$ and $110 \mathrm{MHz}$. The settling time of the MCMHS was measured under different separations of the two hopping frequencies. Figure 5.9 shows the response for the two separations: $4 \mathrm{MHz}$ and $44 \mathrm{MHz}$. From 


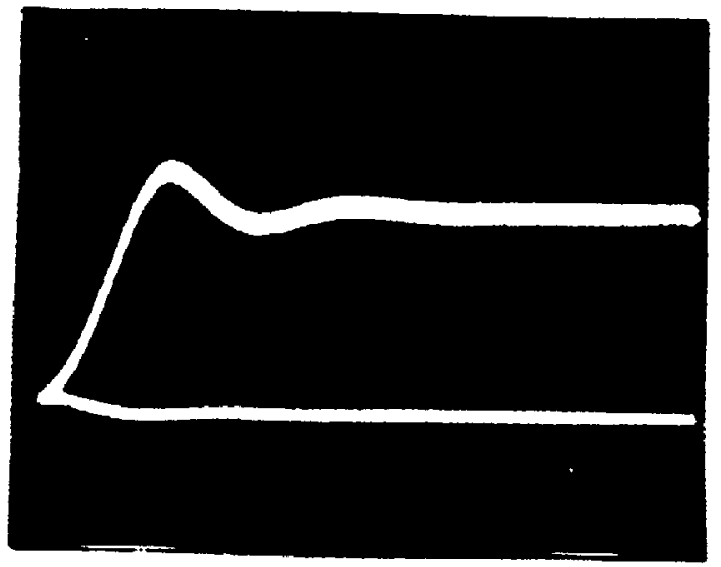

(a)

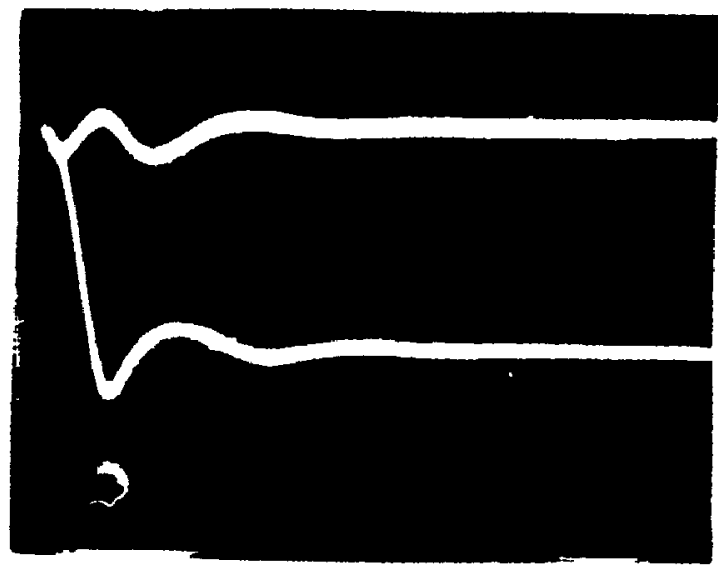

(c)

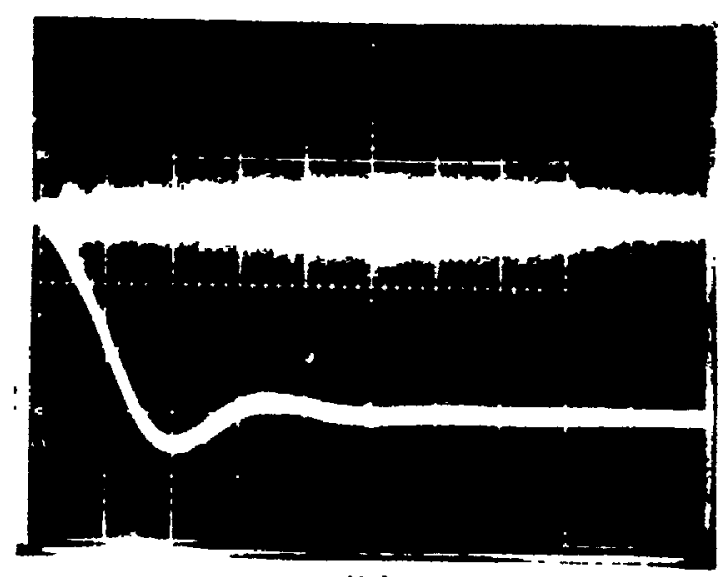

(b)

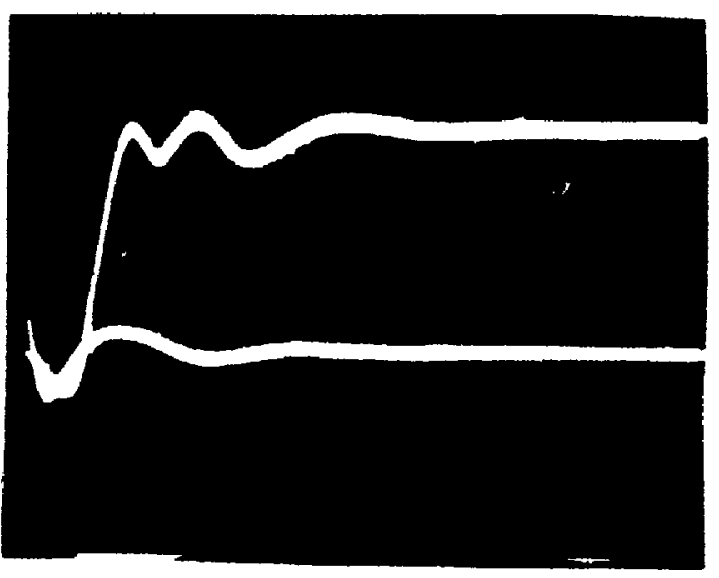

(d)

Figure 5.9: (a): VCO hopping from 1017 to $1021 \mathrm{MHz}$; (b): VCO hopping from 1021 to $1017 \mathrm{MHz}$; (c): VCO hopping from 1068 to $1024 \mathrm{MHz}$; (d): VCO hopping from 1024 to $1068 \mathrm{MHz}$. For (a) and (b): Horizontal: $0.2 \mu \mathrm{sec} / \mathrm{div}$, Vertical: 20 $\mathrm{mV} / \mathrm{div}$; For (c) and (d): Horizontal: $0.2 \mu \mathrm{sec} / \mathrm{div}$, Vertical: $50 \mathrm{mV} / \mathrm{div}$ 
these pictures it is seen that the system settles to $0.01 \%$ within $1.5 \mu$ sec. The second trace in the pictures represents just a reference indicating the magnitude of the voltage step.

(e) DAC Subsystem Verification

By establishing various logic levels in the DAC input and connecting a precision voltmeter at the op-amp output, the DAC subsystem was tested. The DAC was calibrated by adjusting the offset and gain potentiometers shown in Appendix 5.2. An acceptable resolution of $0.20 \mathrm{mV}$ was obtained by recording values of voltage outputs for digital words differing by 1 LSB all over the full output voltage range (0-10 V) of the DAC.

\subsubsection{Selected Hopping Patterns}

The system was set to operate in the open loop mode with no external disturbance. To observe the behaviour of $\hat{\sigma}$-versus-p curves several frequency patterns were selected. The frequency patterns were divided into two categories: deterministic and pseudorandom.

(a) Deterministic

Patterns of 2, 4 and 8 frequencies were chosen and two quantities were varied: the distance between the hopping frequencies $\left(d f_{h}\right)$ and the distance between the frequencies and the LO ( $\left.d f_{L O}\right)$. Hence we have 4 different experimental arrangements

Low $d f_{L O}(\sim 5$ to $10 \mathrm{MHz})$, low $d f_{h}$ ( $\sim 5$ to $10 \mathrm{MHz}$ )

High $d f_{L O}(\sim 80 \mathrm{MHz}), \quad$ low $d f_{h}(\sim 5$ to $10 \mathrm{MHz})$

Low $d f_{L O}(\sim 5$ to $10 \mathrm{MHz})$, high $d f_{h}(\sim 50$ to $80 \mathrm{MHz})$

High $d f_{L O}(\sim 80 \mathrm{MHz}) \quad$ high $d f_{h}(\sim 50$ to $80 \mathrm{MHz})$ 
In this way, the effect of increasing $d f_{L o}$ and $d f_{h}$ can be observed.

(b) Pseudorandom

The MCMH synthesizer was set to hop using PN patterns of 25, 50, 75 and 100 frequencies. The hopping frequencies were located in the $1500 \mathrm{MHz}$ region being approximately $1 \mathrm{MHz}$ apart from each other. Figure 5.10 (a) sisows a spectrum analyzer picture of 4 hopping frequencies (approximately $5 \mathrm{MHz}$ apart) while in Figure 5.10(b) the system was set to hop using a 100 frequencies PN pattern. The whole spectrum covers approximately $90 \mathrm{MHz}$.

\subsubsection{Short-Term Frequency Stability Measurements}

Figure 5.11(a) shows the $\hat{\sigma}^{2}$-versus-p curve for a system hopping between two frequencies with both $d f_{L O}$ and $d f_{h}$ low $\left(f_{L O}=1535 \mathrm{MHz}, f_{1}=1538.4, f_{2}=1544: \mathrm{MHz}\right)$. Figure 5.11(b) is the same curve with the same $f_{20}$ but now $f_{1}$ and $f_{2}$ are located $50 \mathrm{MHz}$ apart. It is seen that there exist flat horizontal portions for both curves. Similar situations were observed for high $d f_{\text {Lo }}$ as well. Although Figure 5.11 is representative of how the curves behave, numerous other curves were obtained for various values of $f_{L O}, f_{1}$ and $f_{2}$. It was noticed that for all two-frequency measurements, the normalized variances $\hat{\sigma}_{y, k}$ remained at the same levels regardless of the $d f_{L O}$ and $d f_{h}$ values. Normalized variances were produced from the absolute variance values shown on the figures by dividing by the nominal count $\left(f_{0} \tau\right)$ for each experiment. Repeatability of the measurements was established by obtained two or more data files with the same conditions during different periods of the day.

Figure 5.12 shows two $\hat{\sigma}^{2}$-versus-p curves for the system hopping between four frequencies, with $5 \mathrm{MHz}$ separations. The $\mathrm{LO}$ was set at $1537 \mathrm{MHz}$ while the lowest output frequency was located at $1540 \mathrm{MHz}$. These curves also exhibit horizontal portions. Figuie 5.13(a) depicts two variance curves using two hopping frequencies 


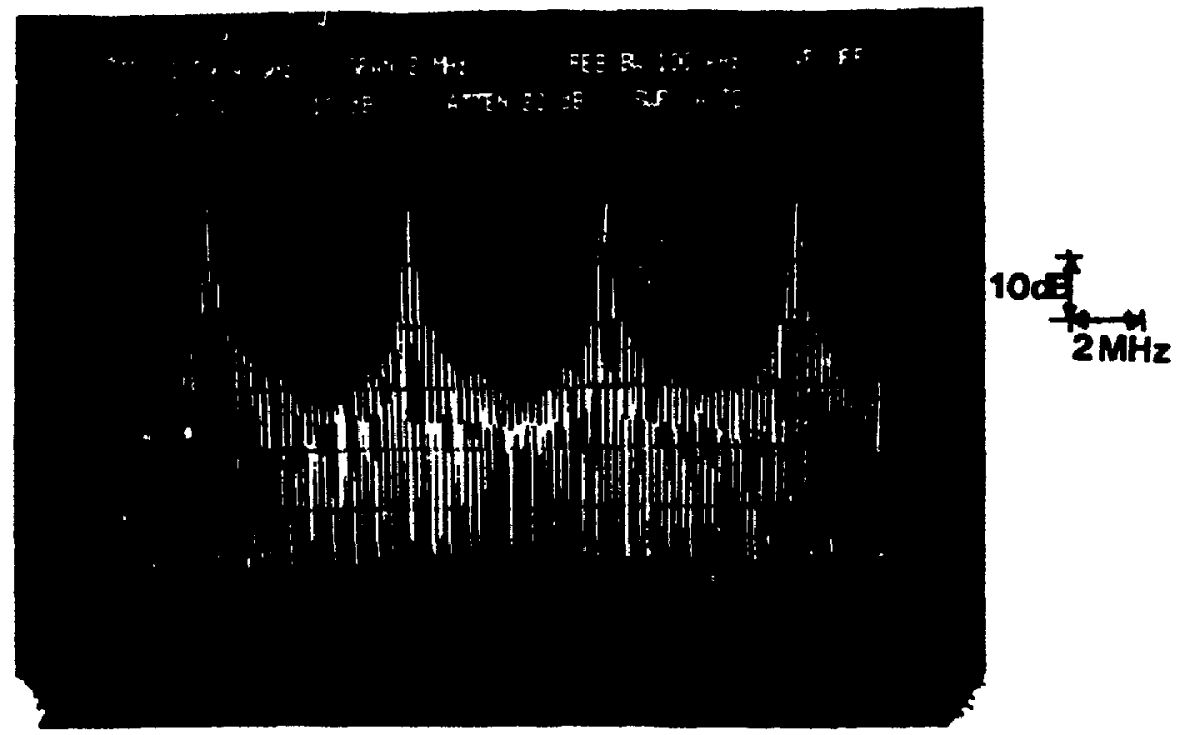

(a)

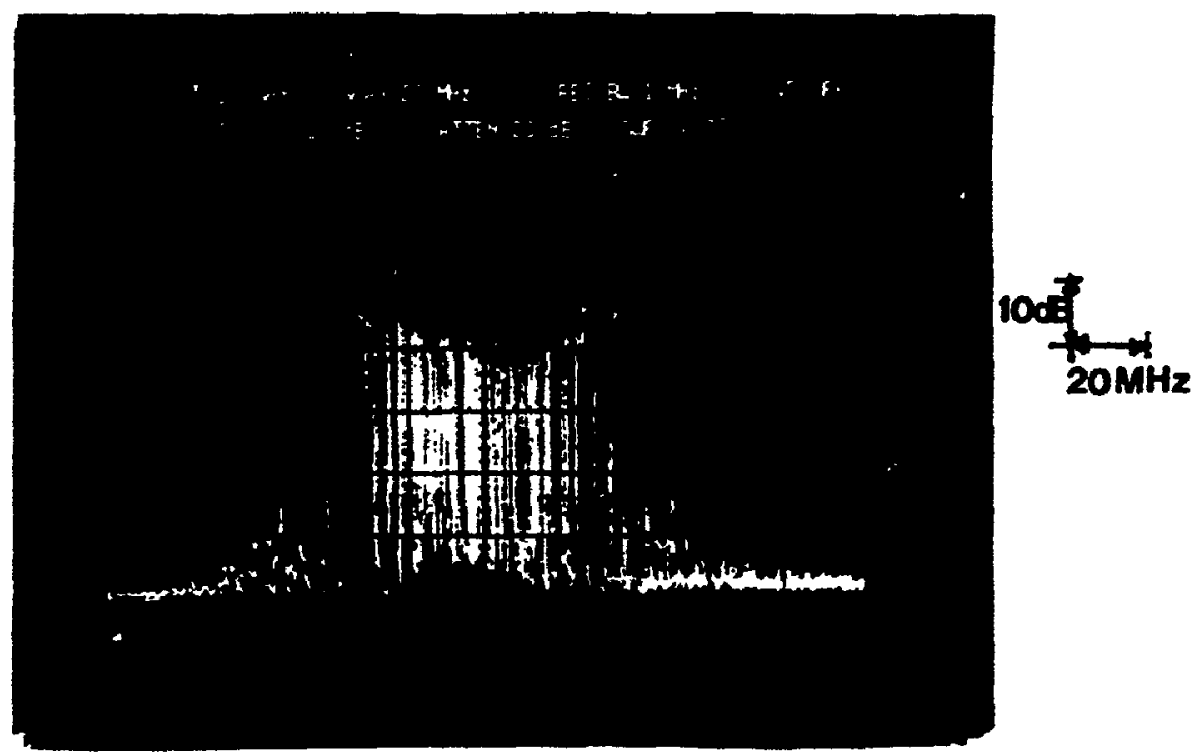

(b)

Figure 5.10: (a): MCMH synthesizer hopping between 4 frequencies; (b): Pseudorandom pattern frequency hopping (100 frequencies) 


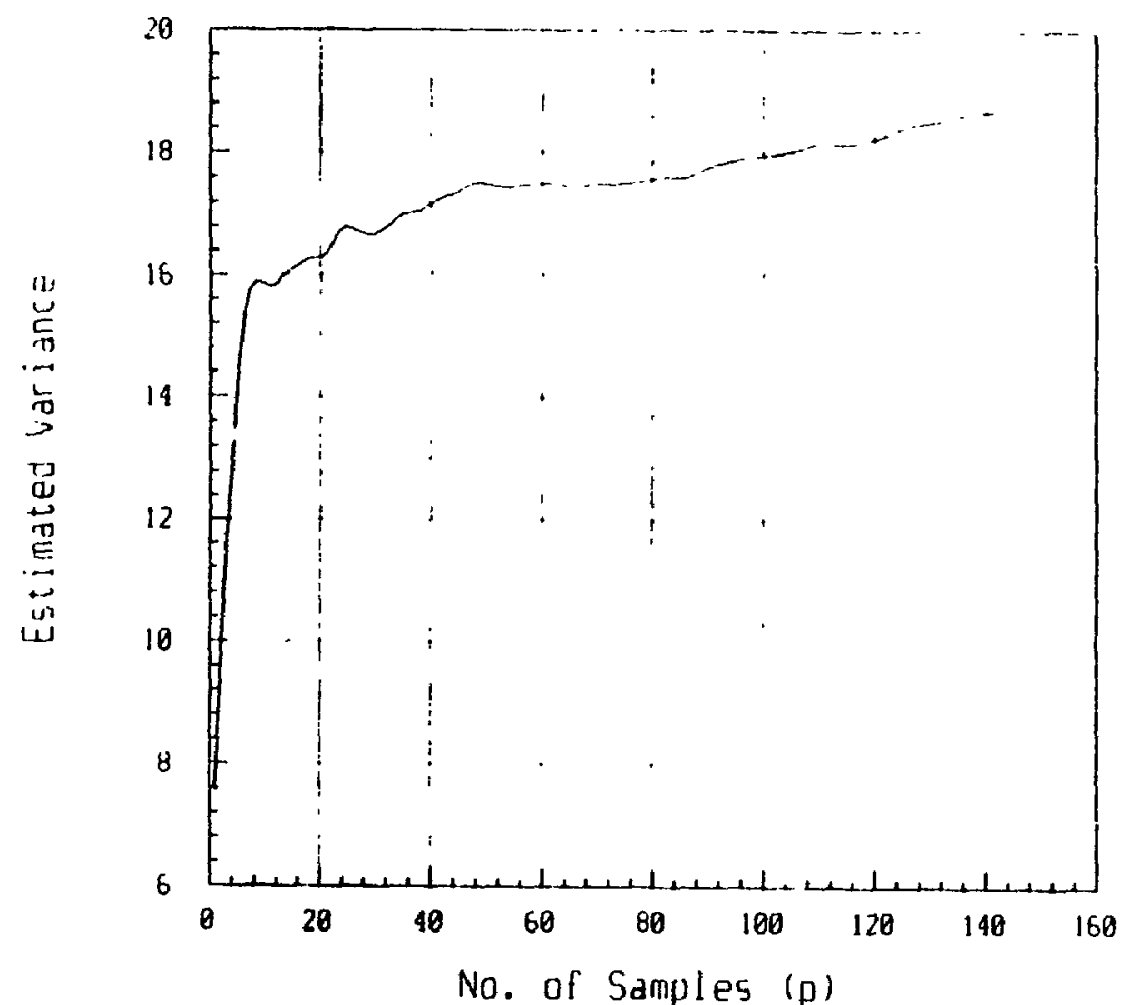

(a)

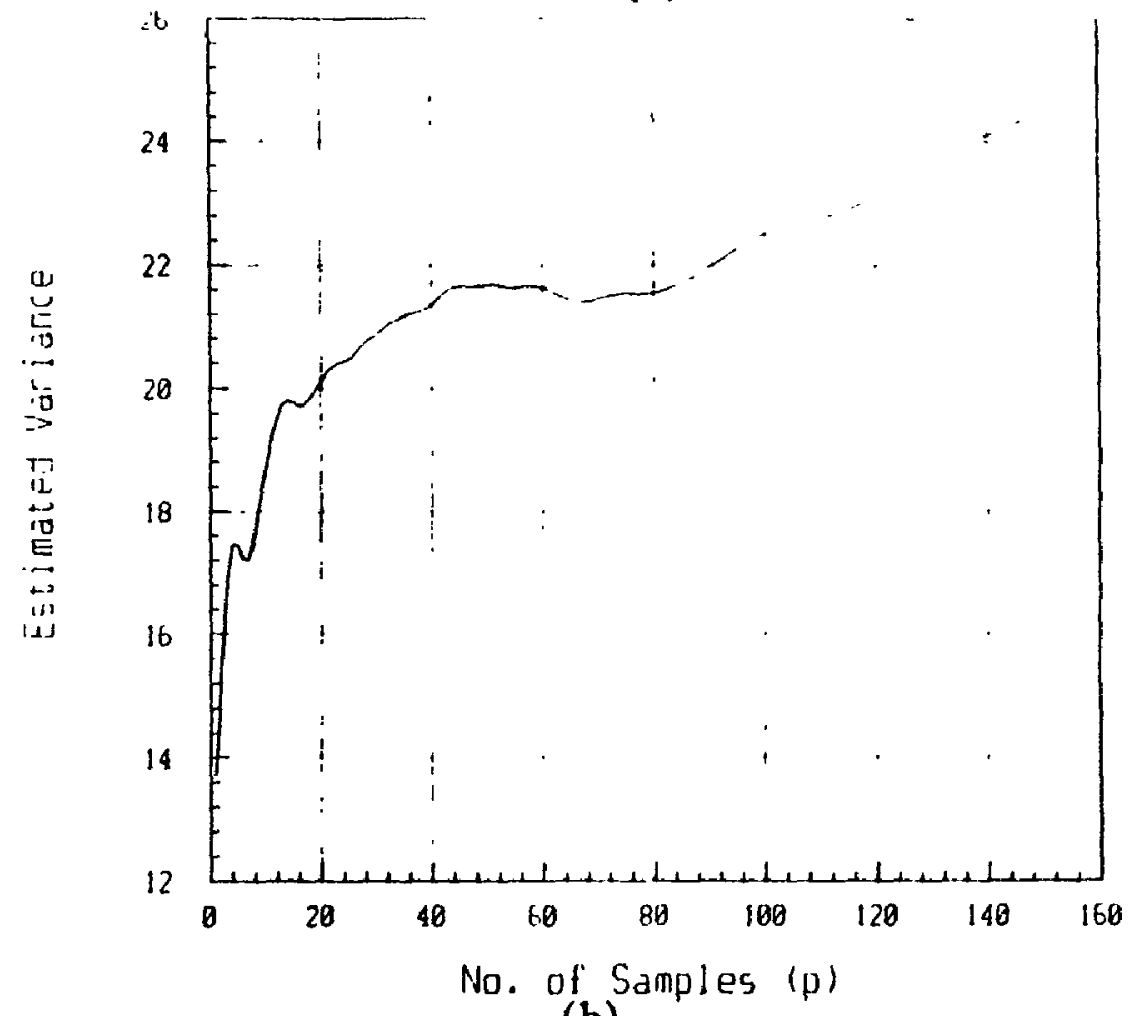

Figure 5.11: (a): $\hat{\sigma}^{2}$-versus- $p$ for system hopping between two frequencies: $\Delta f=5.5$ MHz; (b): $\hat{\sigma}^{2}$-versus-p curve for system hopping between two frequencies: $\Delta f=50$ $\mathrm{MHz}$ 


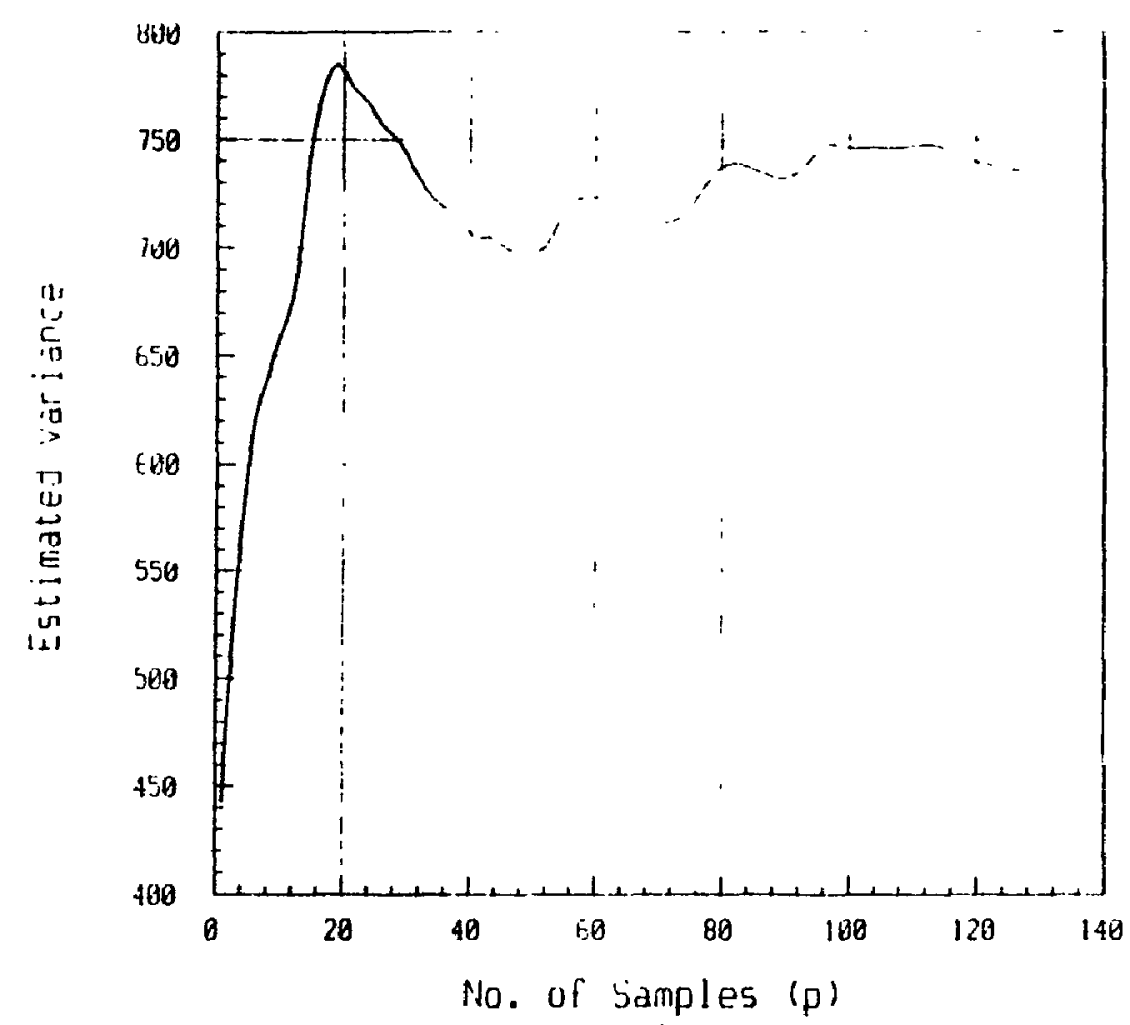

(a)

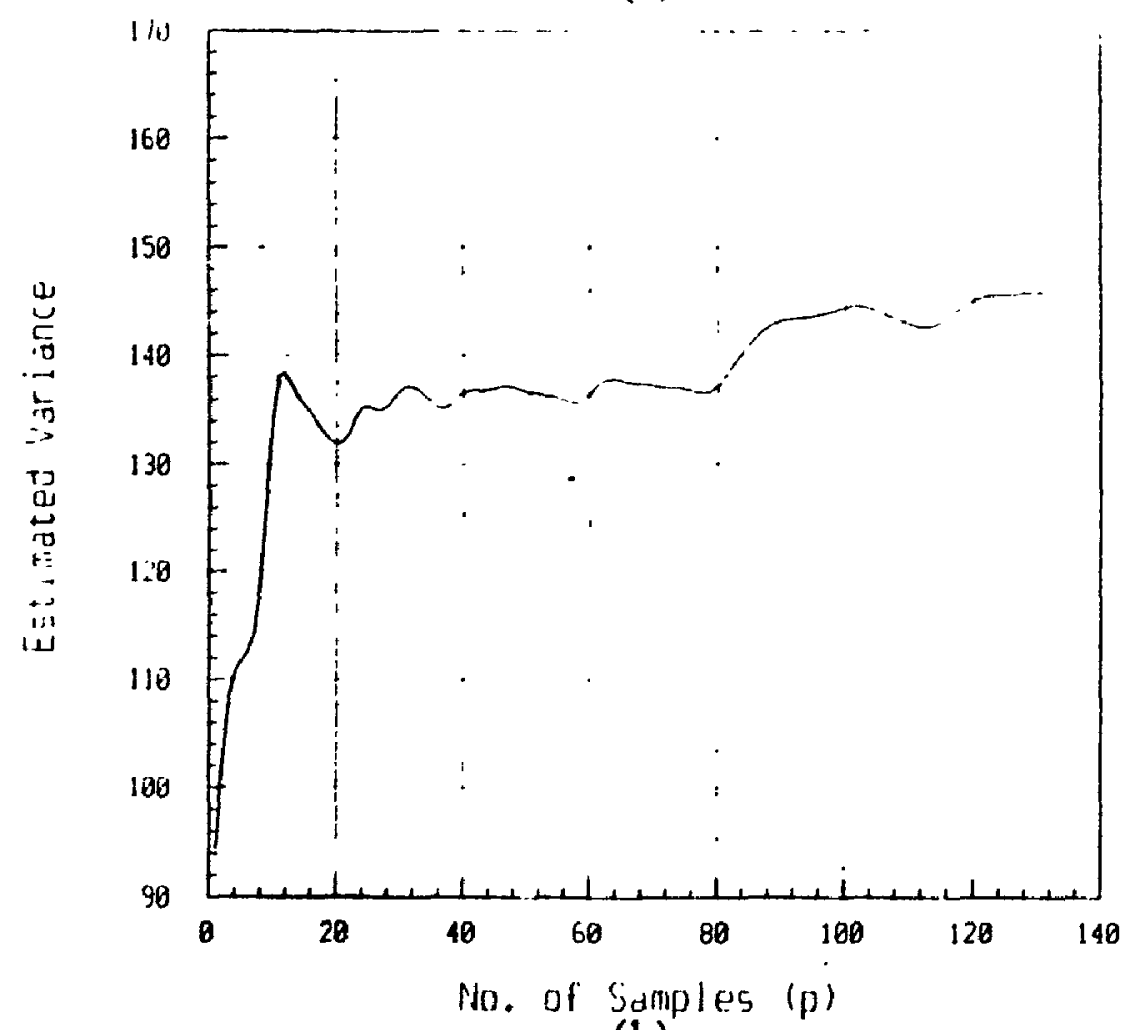

(b)

Figure 5.12: $\hat{\sigma}^{2}$-versus-p curves for system hopping between 4 frequencies with $\Delta f=5 \mathrm{MHz}$; (a) Data from $f_{1}=1540 \mathrm{MHz}$ were used; (b) Data from $f_{2}=1550$ $\mathrm{MHz}$ were used. 


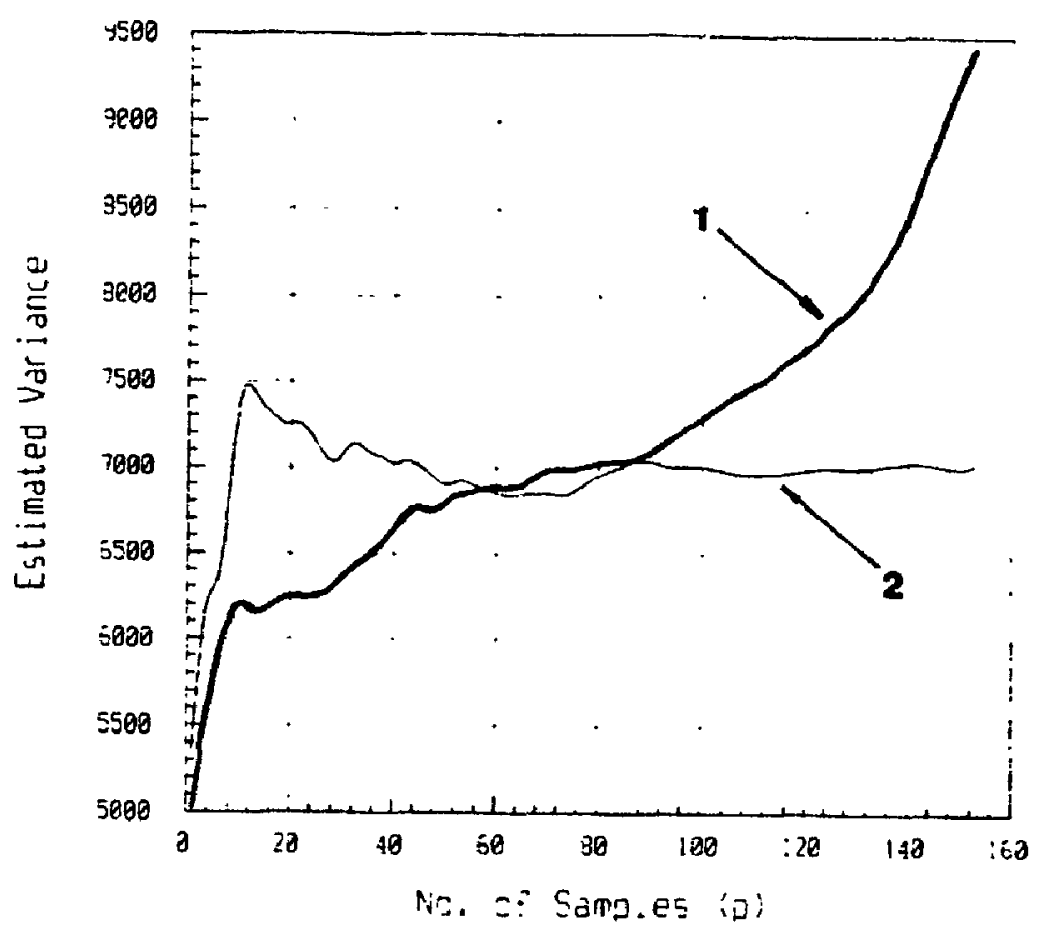

(a)

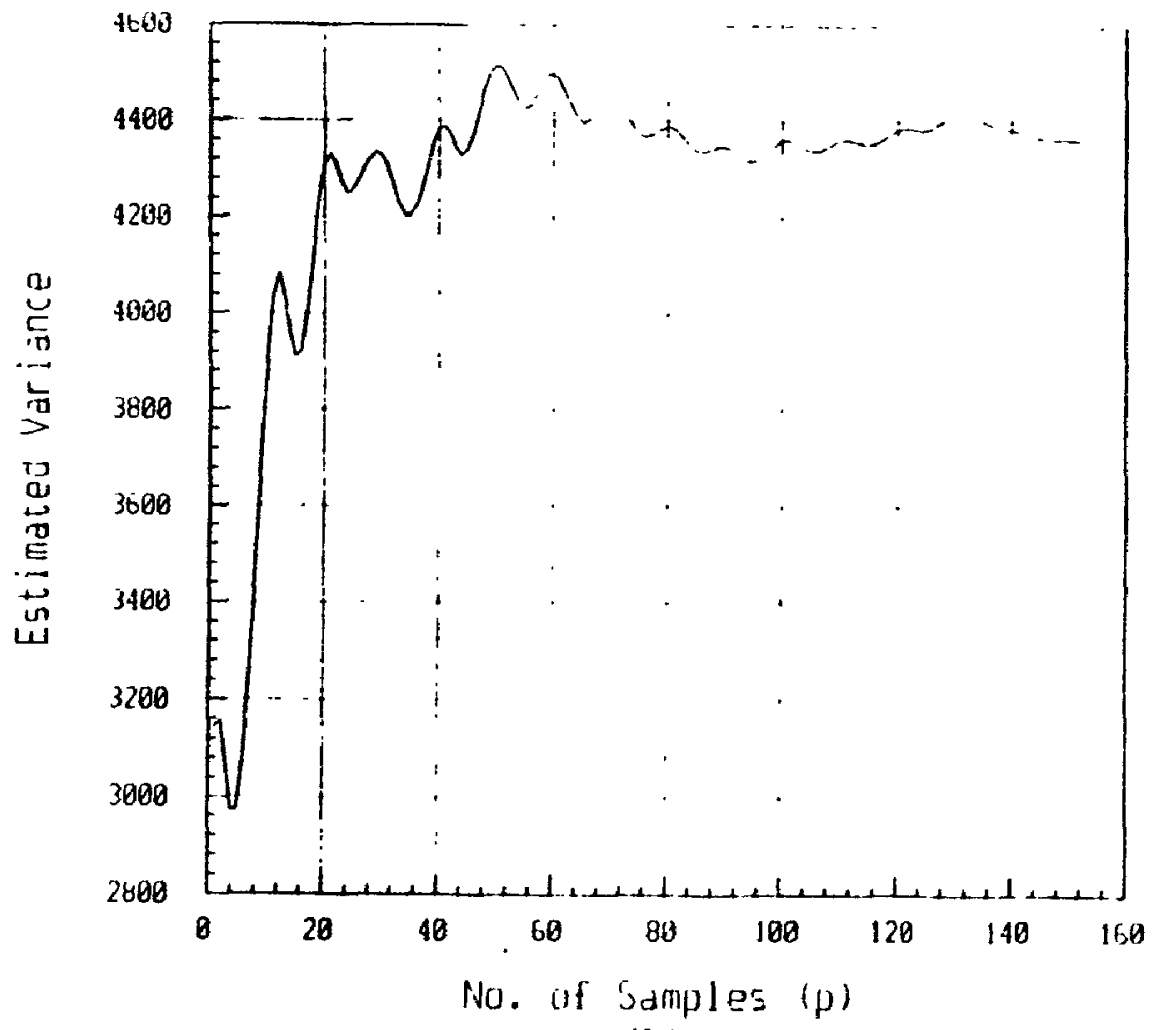

(b)

Figure 5.13: (a): $\hat{\sigma}^{2}$-versus-p curves with and without external disturbance to the VCO; (b) $\hat{\sigma}^{2}$-versus-p for PN frequency hopping (75 frequencies) 
and the following conditions: $f_{L O}=1470.0 \mathrm{MHz}, f_{1}=1536 \mathrm{MHz}, f_{2}=1540 \mathrm{MHz}$. The only difference is that a very slow drift was introduced in the first experiment (curve 1) involving an overall frequency drift of $\pm 600 \mathrm{KHz}$ within 40 minutes. This was done by successively heating and cooling the VCO. The second curve was obtained while the VCO was not disturbed by any external source. One can see that although curve 1 diverges quickly after $p \simeq 90$, it still exhibits a short horizontal part producing the same variance level as the undisturbed system (curve 2). Finally, Figure 5.13(b) shows the variance curve of the VCO while it is set to hop pseudorandomly among 75 frequencies. Again the horizontal portion is present.

From all the experiments involving 2, 4, 8, 25, 50, 75 and 100 frequencies it was found that the values of the normalized variance increase by approximately $10 \%$ each time the number of frequencies is doubled.

\subsubsection{Real-Time System Adaptation Measurements}

The system was set in the closed-loop mode and severe frequency offset was introduced by adjusting the fine voltage tuning of the dc bias of the VCO. This produces an offset of approximately $2 \mathrm{MHz}$ within $10 \mathrm{sec}$. The system was commanded to adjust its frequency cumulatively after the $10 \mathrm{sec}$. time interval. This means that no matter what the offset is during these $10 \mathrm{sec}$, at $t=10 \mathrm{sec}$. the frequency should go back to where it should be. A simple LMS algorithm was used for adaptation. Figure 5.14(a) demonstrates the outcome as shown on a spectrum analyzer. The leftmost curve represents the output frequency $f_{r}$ at $t=0_{t}$. At $t=0_{+}$, a linear offset was introduced which pushed $f_{r}$ slowly to the right by about 4 divisions ( $2 \mathrm{MHz}$ ). At $t=10 \mathrm{sec}$ the algorithm adjusts the system and brings the frequency back. The rightmost curve indicates the position of the adjusted output frequency. The two peaks differ by a distance close to $50 \mathrm{KHz}$. 


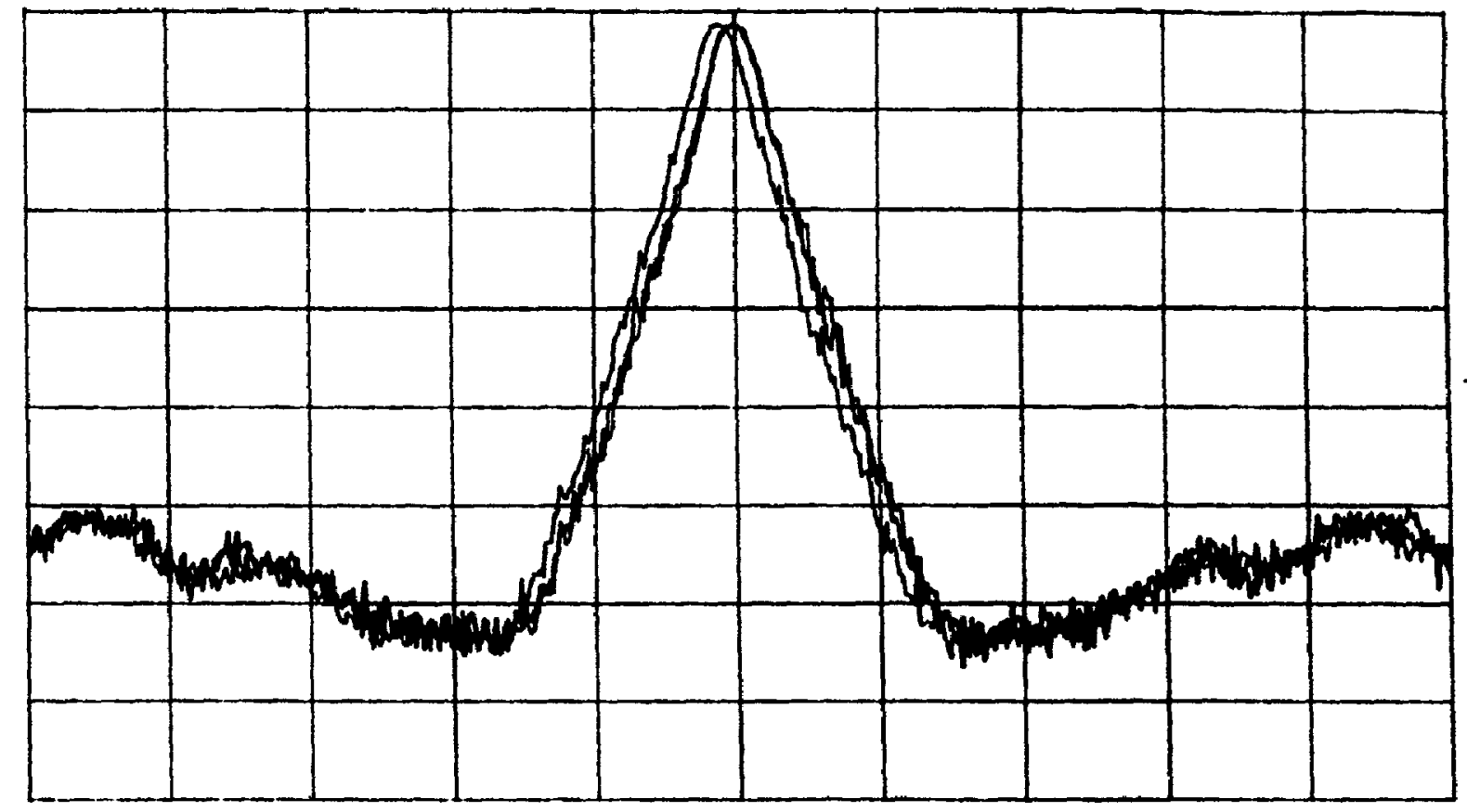

(a)

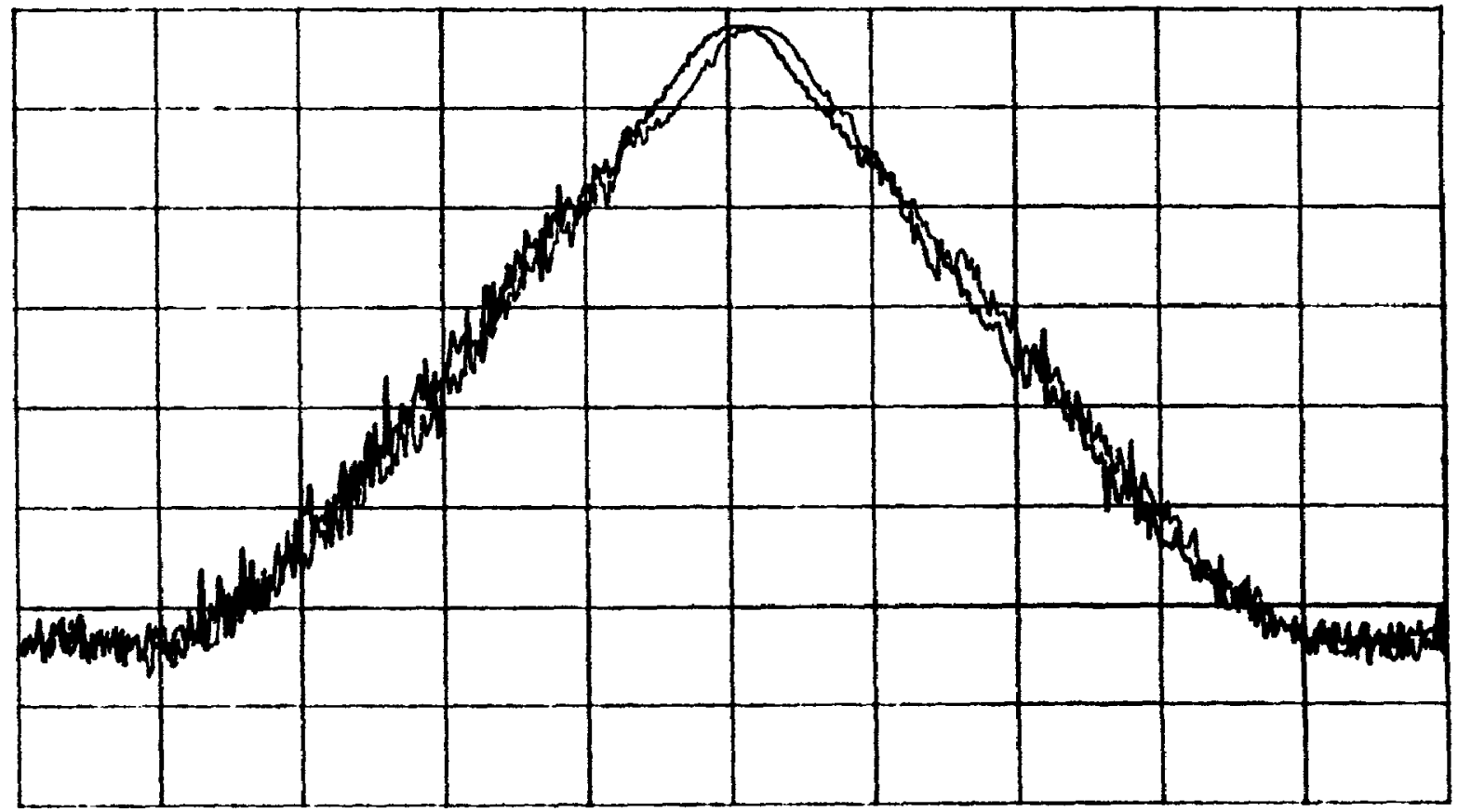

(b)

Figure 5.14: (a): Initial and final frequency using adaptation (linear offset); $(\mathrm{CTR}=1.5368 \mathrm{GHz}$ Horizontal $=500 \mathrm{KHz} / \mathrm{div}$ Vertical $=10 \mathrm{~dB} / \mathrm{div})$; (b): Initial and final frequency using adaptation (sinusoidal offset) (CTR=1.5364 GHz, Horizontal $=200 \mathrm{KHz} / \mathrm{div}$, Vertical $=10 \mathrm{~dB} / \mathrm{div}$ ) 
Figure 5.14(b) demonstrates a similar situation. The only difference is that an approximate sinusoidal offset was introduced instead of a linear one. Again the resulting difference is close to $40 \mathrm{KHz}$. Figures 5.15 depict the error between the counted and the predicted value for two different values of the adaptation coefficient $\mu$. In Figure 5.15(b) $\mu$ is lower than that in Figure 5.it5(a). As a result the MSE is lower than the one in Figure 5.15(a) and the convergence is slower as is theoretically expected. Finally, Figure 5.16 depicts the bebaviour of the algorithm for value of $\mu$ higher than the one demanded by the stability criterion. It is seen that the algorithm diverges and the error becomes as large as it can be $(\sim 32,000)$. 


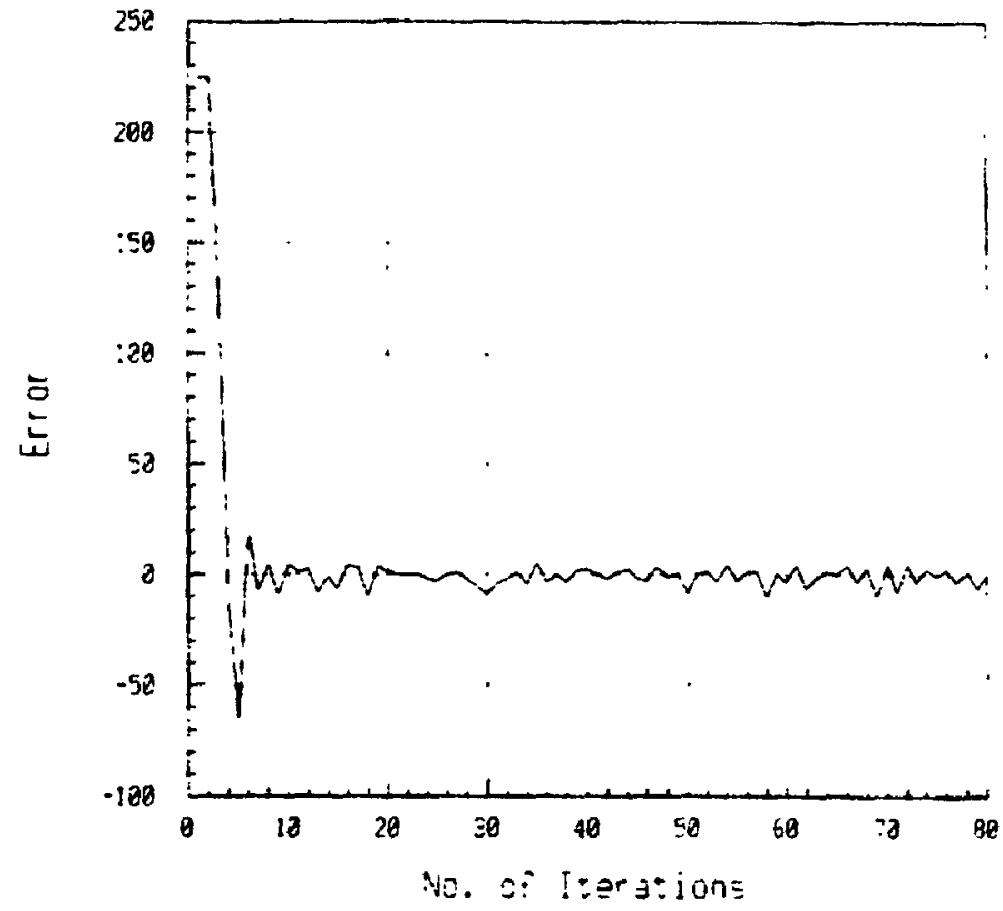

(a)

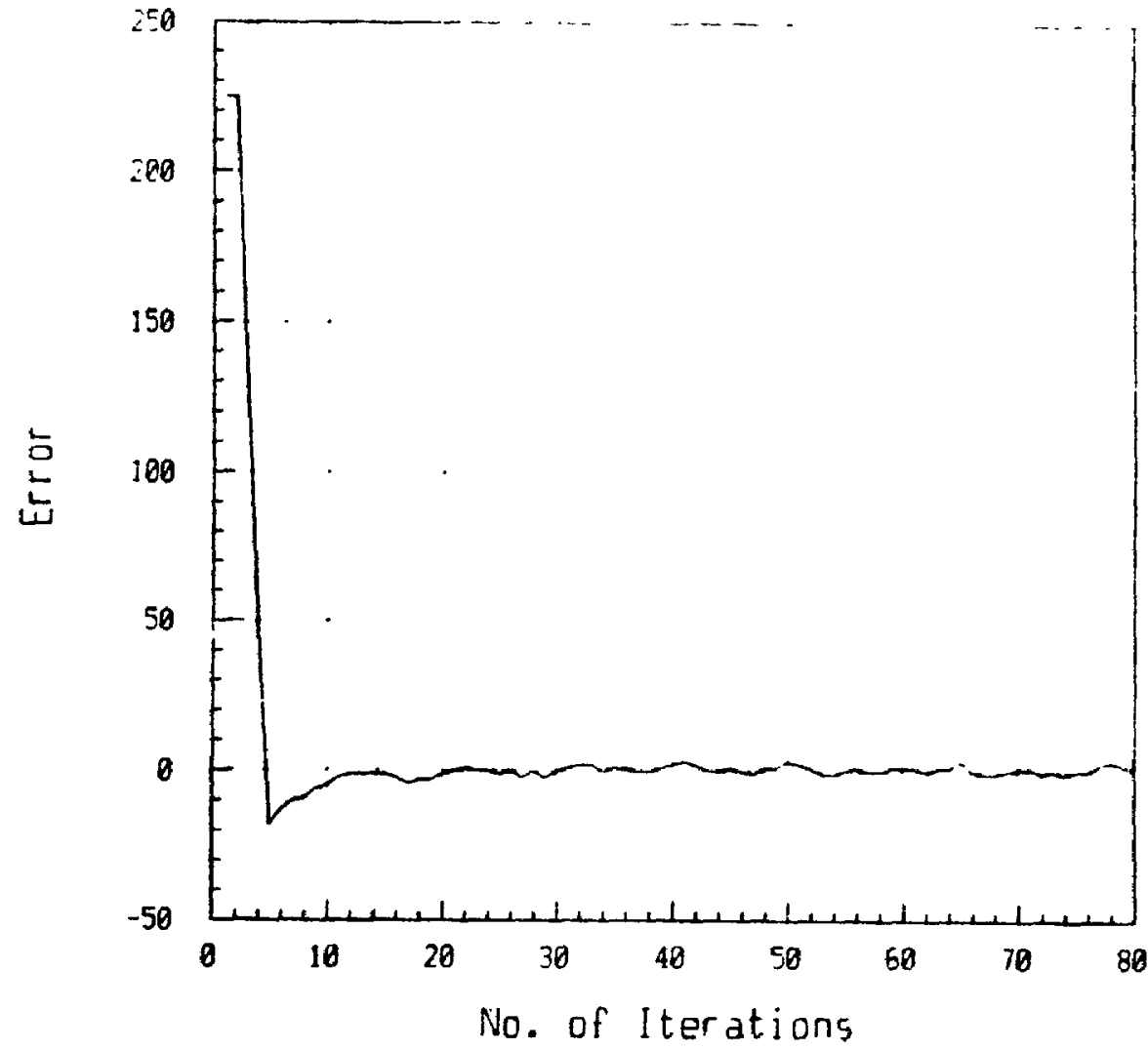

(b)

Figure 5.15: (a): Error between predicted and actual counts from LMS $\left(\mu=8 \times 10^{-6}\right)$; (b) Error between predicted and actual counts from LMS $\left(\mu=1 \times 10^{-6}\right)$ 


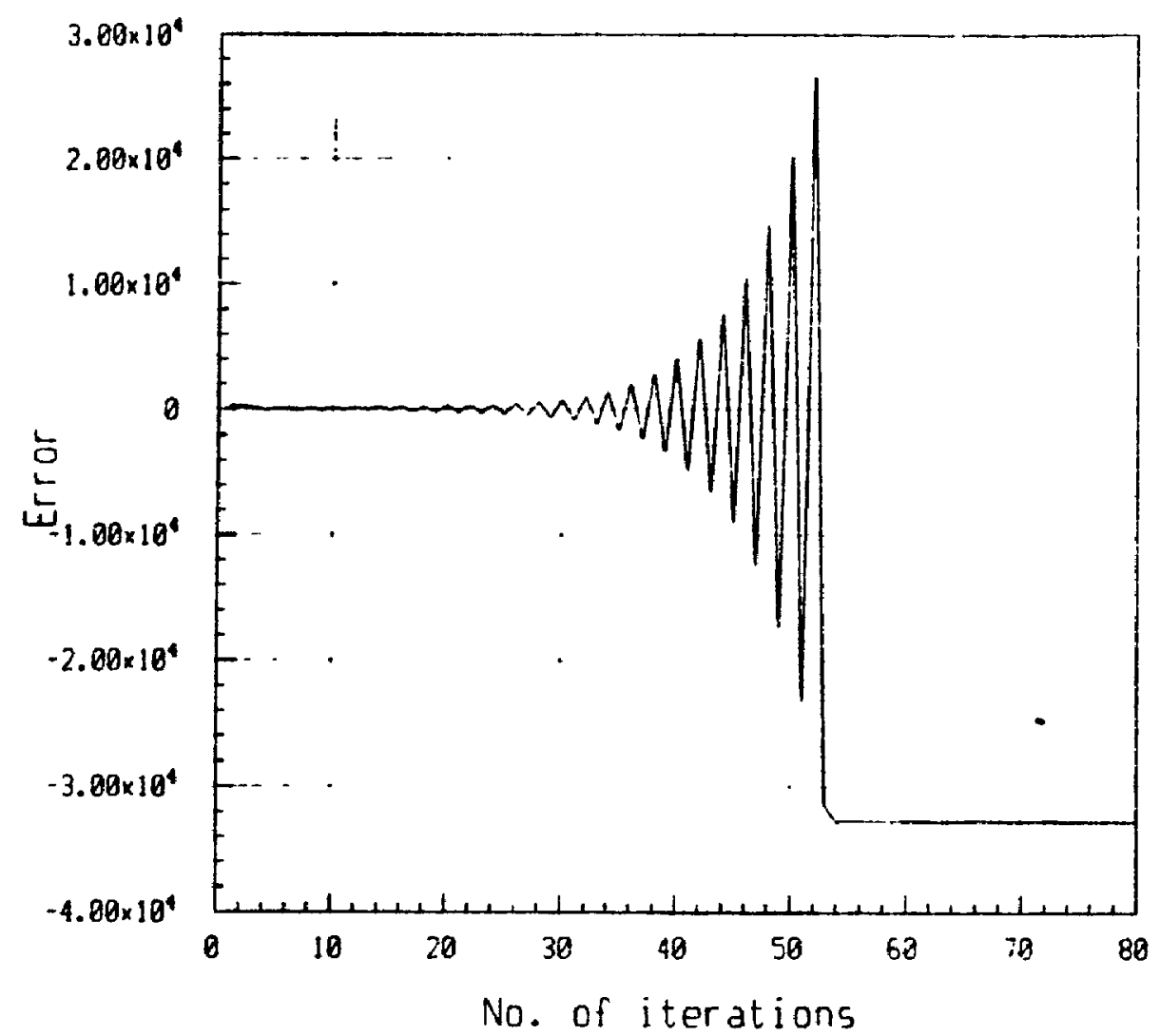

Figure 5.16: Error when $\mu=1.3 \times 10^{-5}$, creating instability 
Appendix 6.1

TYPICAL VCO CHARACTERISTICS SUITABLE FOR A $500 \mathrm{MHZ}$ FREQUENCY SYNTHESIZER

\begin{tabular}{|c|c|c|c|c|}
\hline Model & $\begin{array}{c}\text { Frequency } \\
\text { Range } \\
\text { (GHz) }\end{array}$ & $\begin{array}{c}\text { Power } \\
\text { Output (dBm) } \\
\text { Min. }\end{array}$ & $\begin{array}{c}\text { Thning } \\
\text { Voltage (VDC) } \\
\text { Max. }\end{array}$ & $\begin{array}{c}\text { Input } \\
\text { Capacitance } \\
\text { Typ. (pf) }\end{array}$ \\
\hline WJ-V801 & $1.5-2.5$ & 13 & $\overline{55}$ & 100 \\
\hline Avantel HTO-2600 & 2.6-5.2 & 10 & 20 & 55 \\
\hline WJ-V201 & $1.0-2.0$ & 10 & 50 & 100 \\
\hline EMF-VTW-4D & $1.0-1.5$ & 9 & 15 & \\
\hline Avantek VTO-8090 & $0.9-1.6$ & 13 & 56 & 180 \\
\hline Model & $\begin{array}{c}\text { All } \\
\text { Harmonics }(\mathrm{dBc}) \\
\text { Typ. }\end{array}$ & $\begin{array}{c}\text { Phase Noise } \\
\text { at } 100 \mathrm{KHz} \\
\text { From Carrier } \\
\text { Typ. (dBc/Hz) }\end{array}$ & $\begin{array}{l}\text { Pulling } \\
\text { All Phases } \\
\text { Typ. } \\
\text { (MHz) }\end{array}$ & $\begin{array}{c}\text { Pushing } \\
\text { (MHz/V) } \\
\text { Typ. }\end{array}$ \\
\hline WJ-V801 & 18 & 110 & $\overline{35}$ & $\overline{3}$ \\
\hline Avantek HTO-2600 & 12 & 95 & 18 & 25 \\
\hline WJ-V201 & 15 & 100 & 25 & 30 \\
\hline EMF-VTW-4D & 15 & 112 & & 20 \\
\hline Avantek VTO-8090 & 15 & $60^{1}$ & 25 & 6 \\
\hline
\end{tabular}

$1-50 \mathrm{KHz}$ away from carrier 


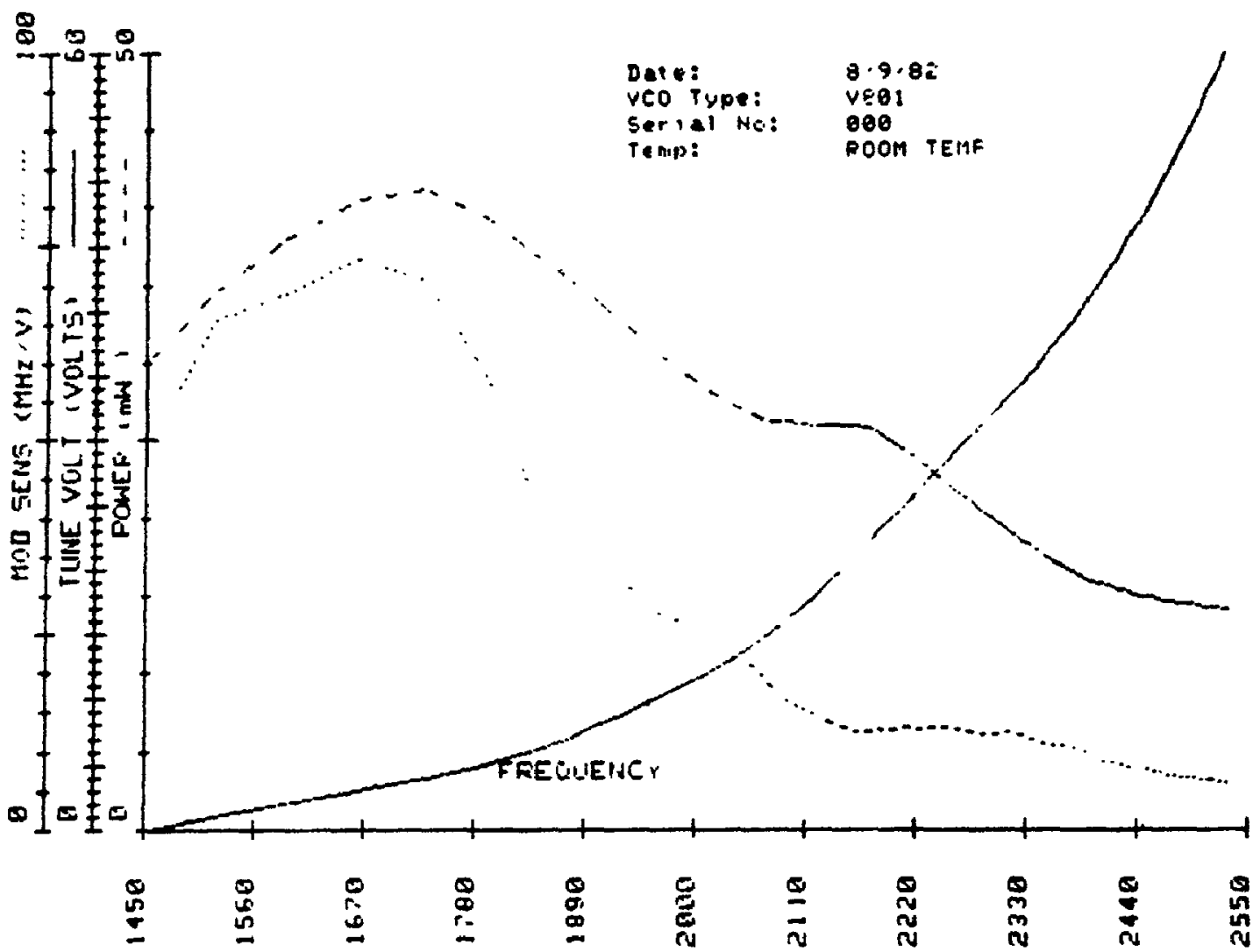

TYPICAL FM NOISE PERFORMANCE - U:I-V801

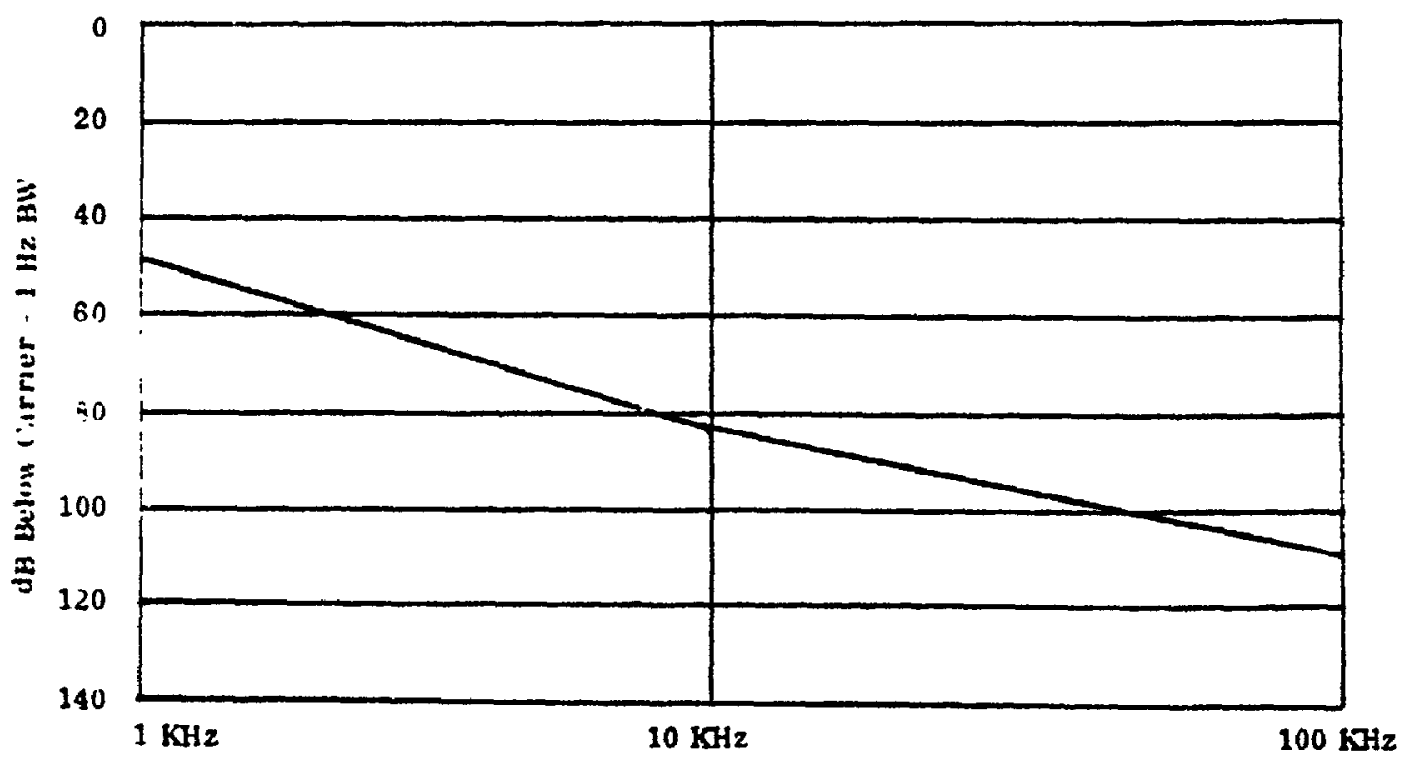

Frequency Offset From Carrier

Figure 5.17: Typical performance of WJ-V801 

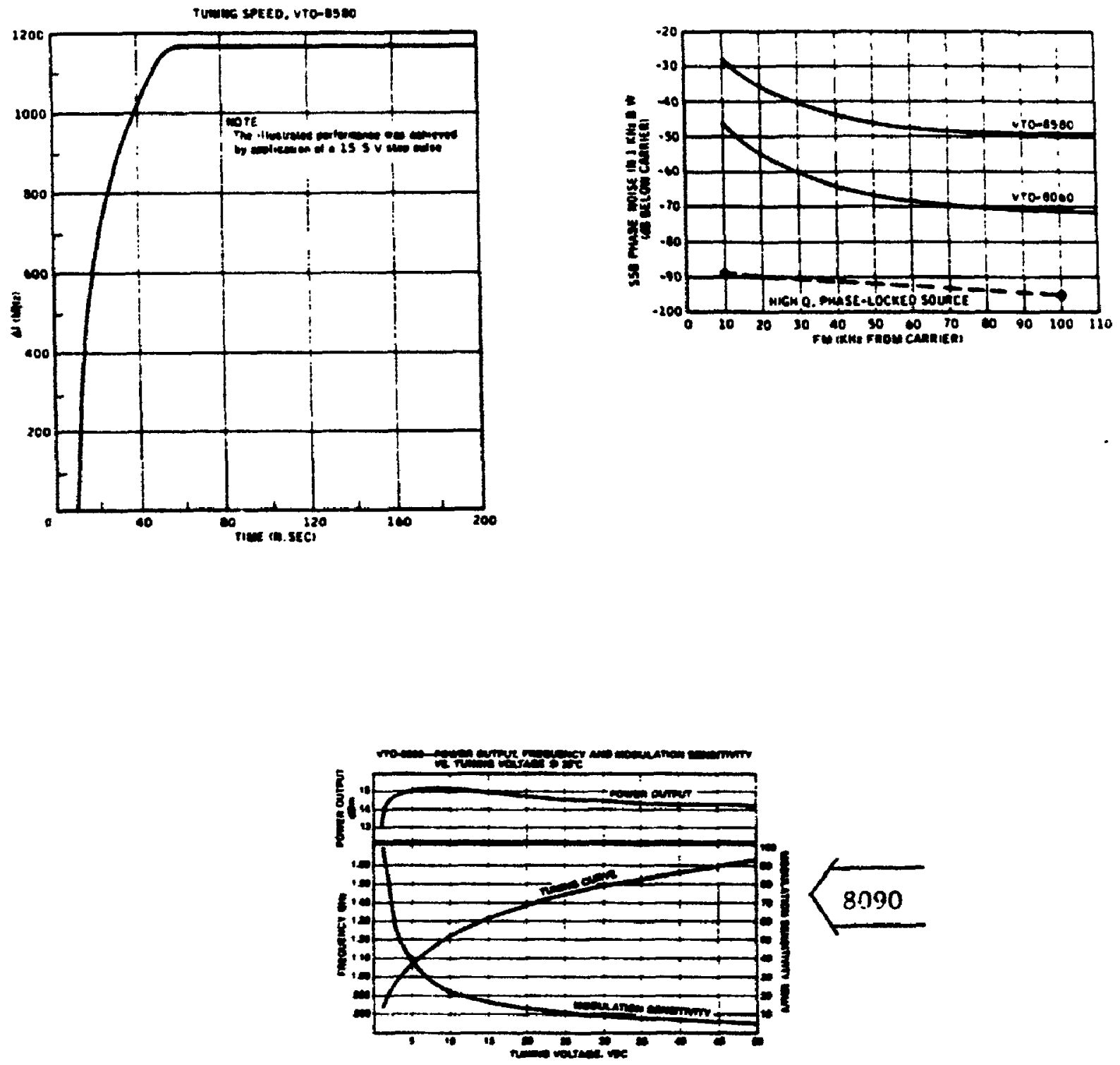

Figure 5.18: Typical performance of VTO-8090 


\section{Appendix $\mathbf{5 . 2}$}

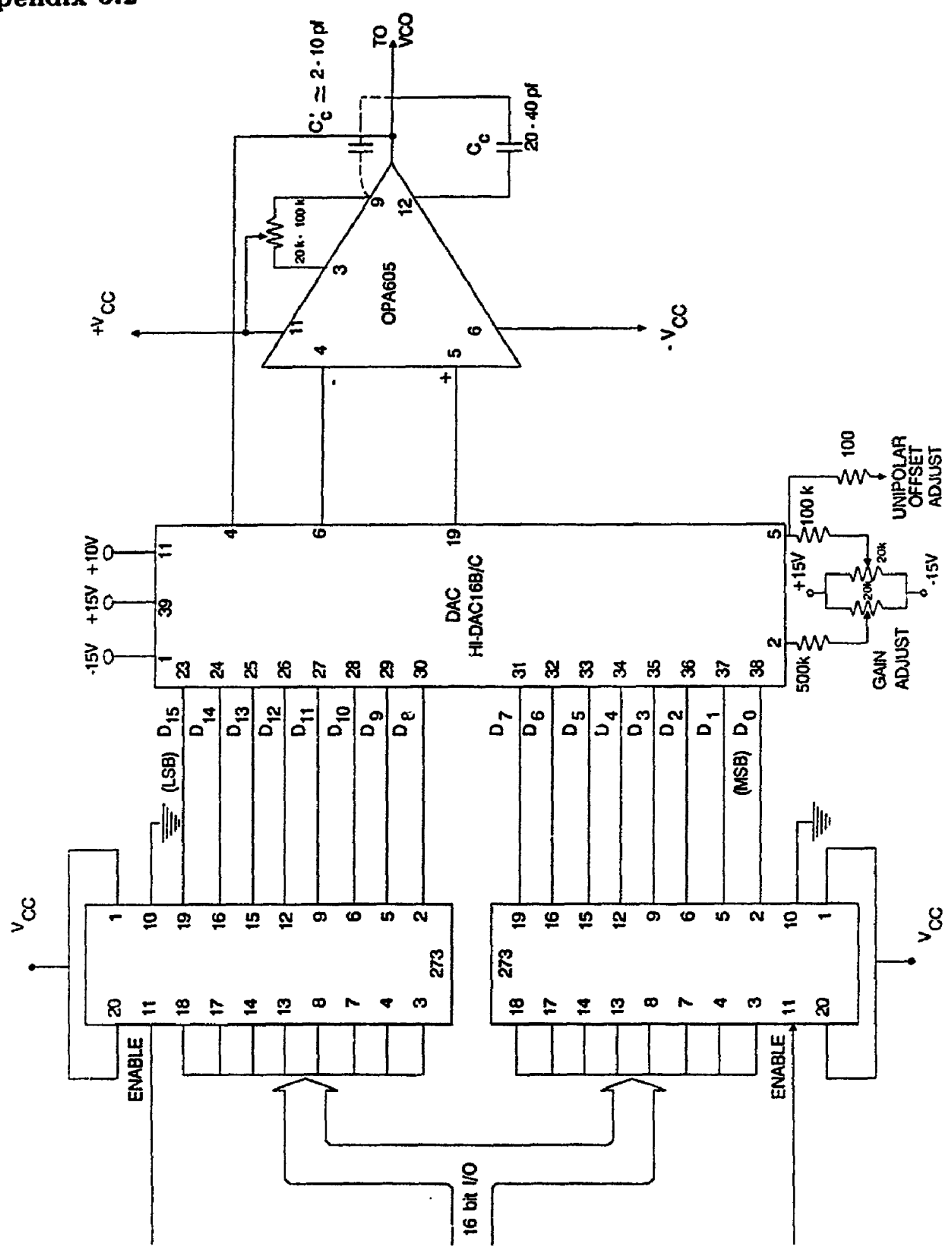

Figure 5.19: The DAC subsystem 


\section{Appendix 5.3}

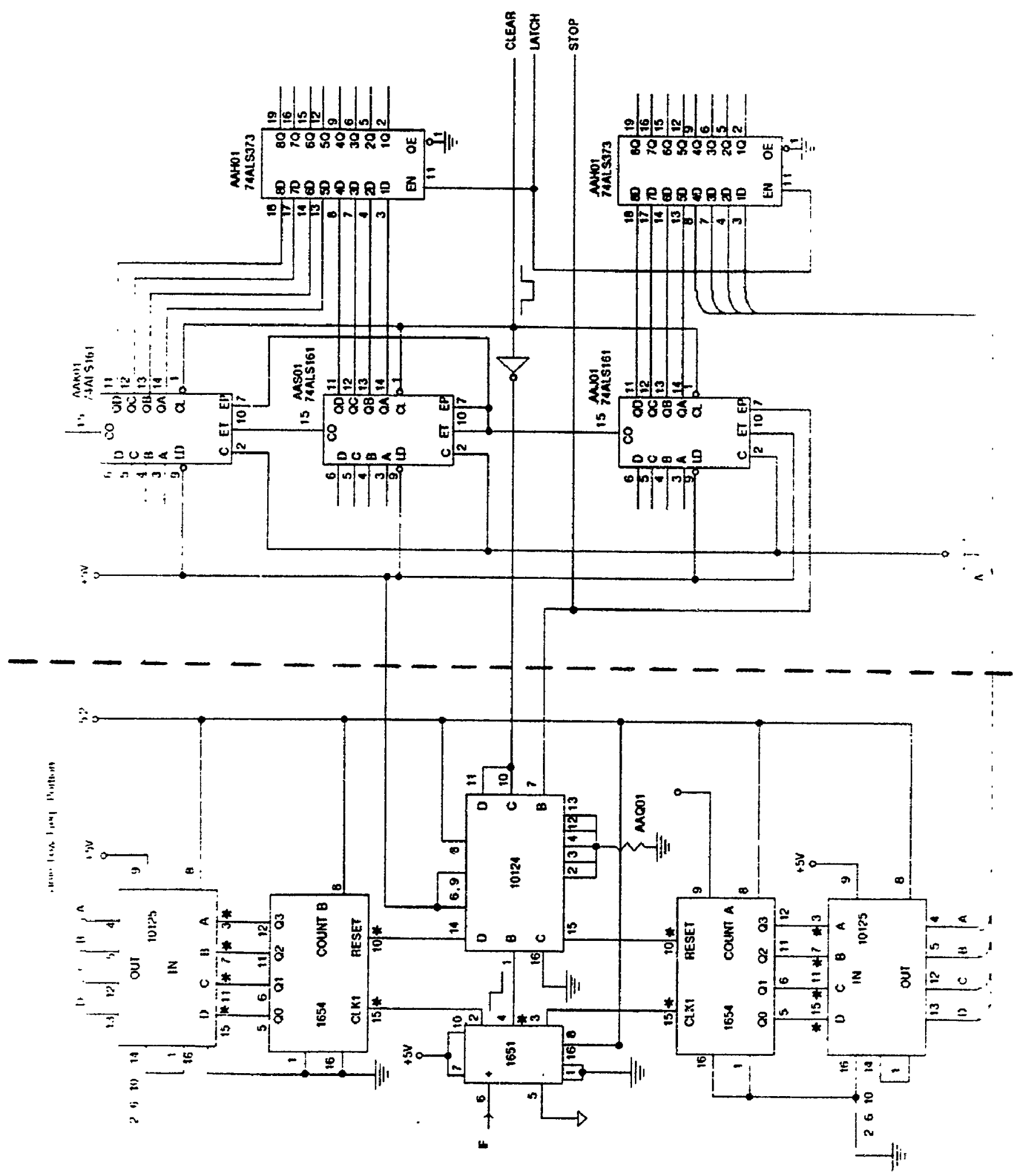

Figure 5.20: The high frequency counter (dashed line separates TTL from ECL) 


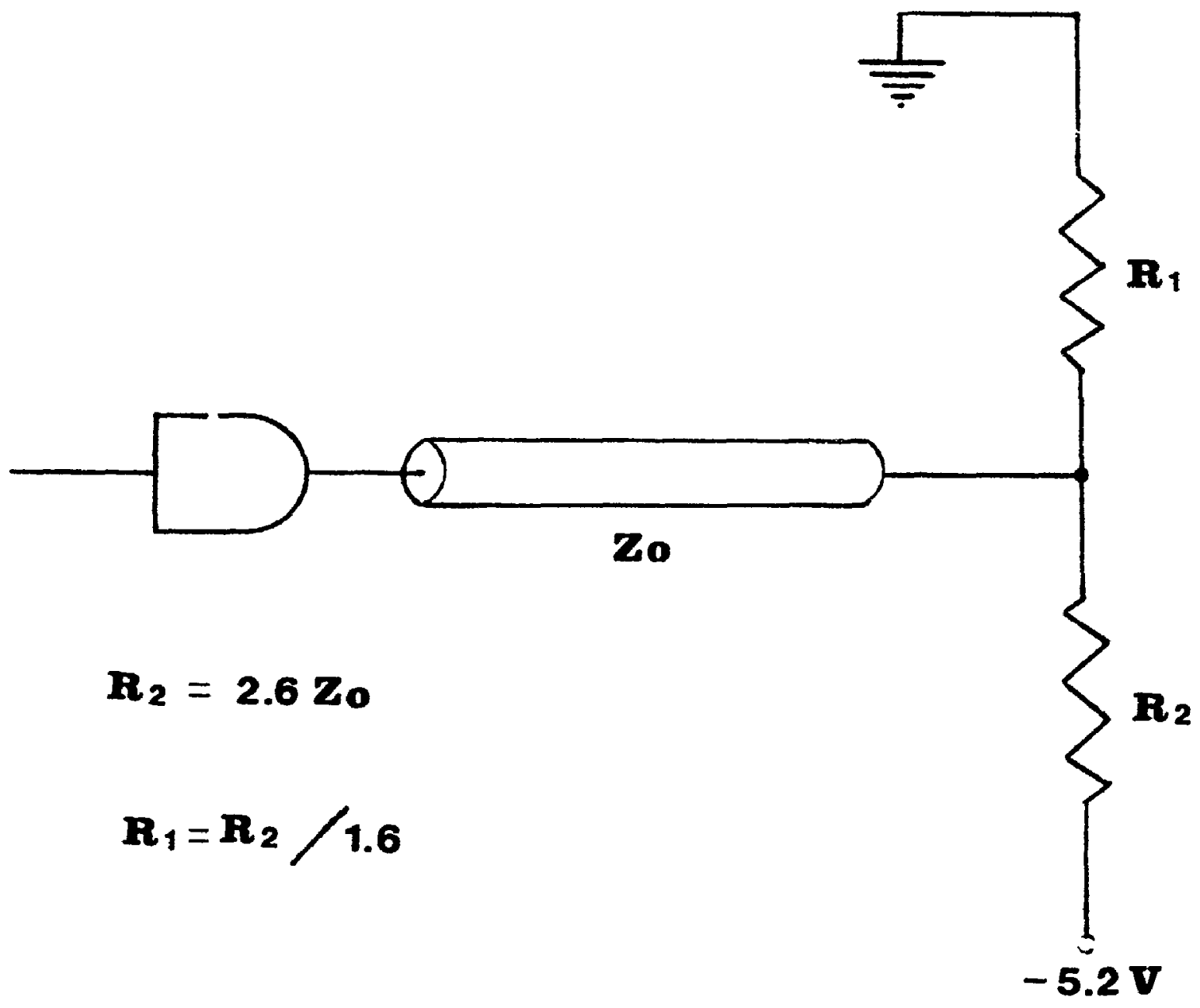

Figure 5.21: Parallel terminating scheme 
Appendix 5.4

THE I/O MICROPROCESSOR INTERFACE

Physical Address Locations Assigned to 8255 Ports

$\begin{array}{llll}300 & \text { 8255A } & \text { Port A } & R / W \\ 301 & \text { 8255B } & \text { Port A } & R / W \\ 302 & \text { 8255A } & \text { Port B } & R / W \\ 303 & 8255 B & \text { Port B } & R / W \\ 304 & \text { 8255A } & \text { Port C } & R / W \\ 305 & 8255 B & \text { Port C } & R / W \\ 306 & \text { 8255A } & \text { Control } & \text { W } \\ 307 & \text { 8255B } & \text { Control } & \text { W }\end{array}$

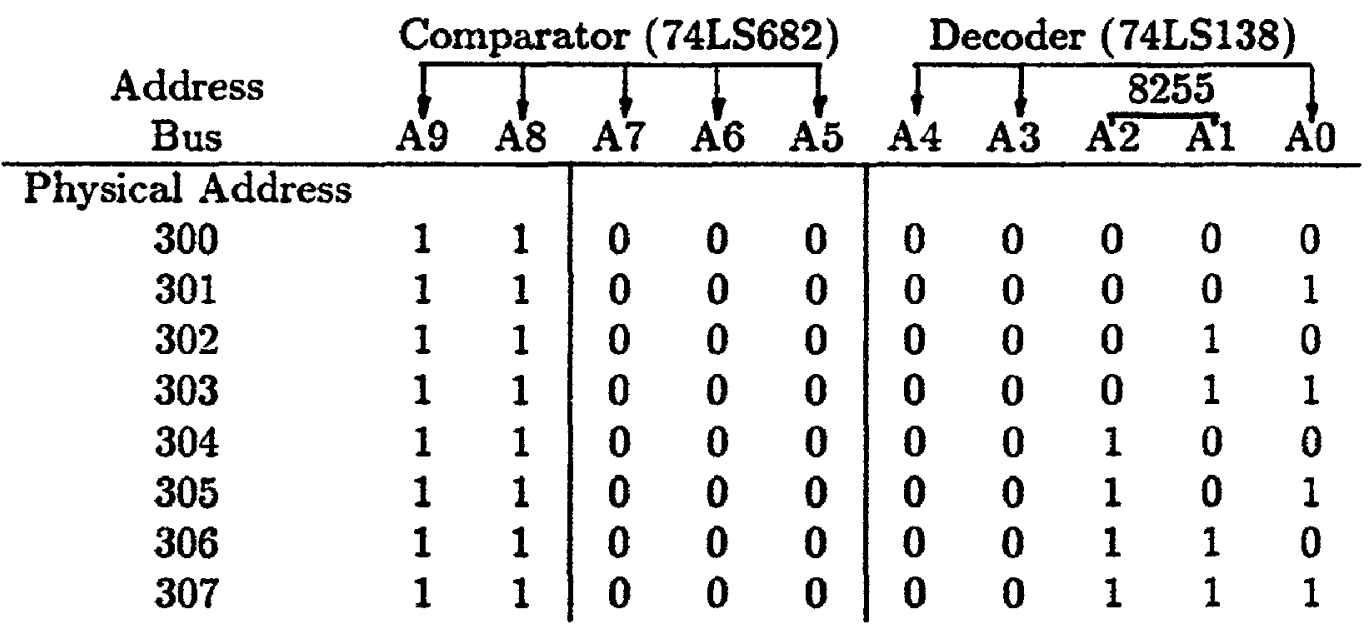




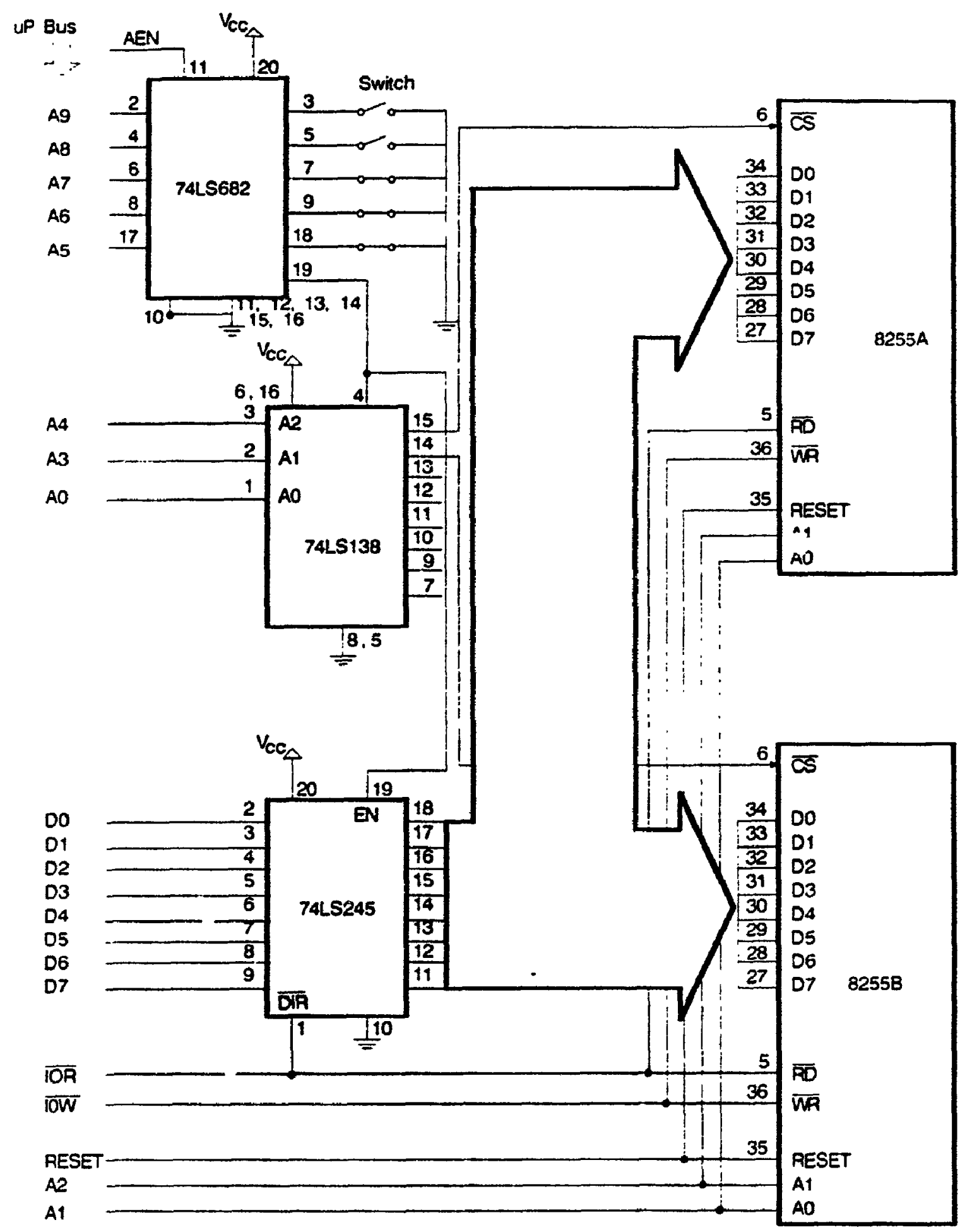

Figure 5.22: Detailed diagram of the $1 / 0$ interface 


\section{Chapter 6}

\section{CONCLUSIONS}

\subsection{Conclusions}

This thesis represents a comprehensive study of the TD stability of oscillators with special emphasis on frequency-hopping signals generated by agile sources such as VCOs. As an application of this characterization, a frequency synthesis method is presented which is particularly attractive for use in FH systems. According to this method a DAC is used to command a VCO, while a $\mu \mathrm{P}$ is the processing and controlling system. A frequency counter is used both as a means for frequency stability characterization and as a feedback element. The latter aspect provides the system with the ability to correct itself when systematic frequency offsets affect the VCO. A procedure to characterize the short term frequency stability is outlined, according to which $\hat{\sigma}_{i}^{2}$-versus-p curves exhibit a separation between short-term fluctuations and slow drifts in the form of flat horizontal parts. A novel approach for obtaining closed-form expressions for TD frequency stability measures is developed and its validity is demonstrated by applying it to the Allan-Barnes variance measure for flicker and random walk frequency noise. The general rule formulated in eqns.(2.51a) and (b) divides the procedure iuto the calculation of two independent terms: a summation term $Z_{k, i}$ which depends only on the measurement processing, 
and an integral term $I_{k, j}$ which depends only upon the type of noise. Since it is a unified structured systematic approach it can be applied to any variance measure for any kind of noise. This method can be very effective when the shape of the filter gain $G_{i}(f)$ in the integral in eqn.(2.12) is chose a priori. This is the transfer function approach.

By enhancing the TD measurement system with real-time processing abilities which take advantage of the use of the frequency counter as a feedback element, a fast-switching frequency synthesizer can be obtained. For this purpose, adaptive algorithms are used to frequency-lock the system with a resolution of approximately $15 \mathrm{KHz}$. This synthesis approach is less complex and very fast compared to the traditional phase locking approach. Compared to direct synthesis methods, it does not exhibit any spurious responses. This makes it attractive for use in ESM systems. In addition, flexibility is an important property of this system since most of its features can be altered by simple changes in the controlling program (dwell time, frequency resolution, output frequency range, different frequency steps and patterns, etc.).

A novel modified LMS algorithm has been outlined. As actually demonstrated by simulations, it is faster than the simple LMS algorithm. This is due to the nature of the usual systematic frequency offset disturbing the VCO.

Finally, the actual system was built and tested under various conditions. From measurements of the TD frequency stability we can conclude that:

(a) For $\tau$ in the region of $100 \mu \mathrm{sec}$ there always exists a flat horizontal portion on the $\hat{\sigma}^{2}$-versus-p curves. This happens regardless of the number of frequencies involved in hopping the spacing between them and the location of the LO frequency used for down conversion. Consequently the PTD, which is a significant factor in the noise of hopping oscillators, does not eliminate the flat region of the curve. 
(b) The only factor affecting the normalized variance level is the number of frequencies. This is to be expected because different frequency steps produce different PSDs. Therefore the higher the number of frequencies, the larger the variety of frequency steps. Hence, for a PN sequence of frequency steps we get a randomly varying short-term PTD. However, within the particular counting interval $\tau$, the integration of this random PTD in the short-term noise of the system still permits us to distinguish flat horizontal parts in the TD stability curves.

The adaptation measurements showed that after an imposed systematic frequency drift of $2 \mathrm{MHz}$, the LMS algorithm corrected the output frequency back to within $40-50 \mathrm{KHz}$ of the nominal value, even in the worst case. This is equivalent to a final offset of approximately 3 bits on the DAC since we know that the resolution of the system is close to $15 \mathrm{KHz}$. It is also found that the behaviour of the adaptive algorithm is as theoretically expected: for a smaller adaptation coefficient $\mu$ it converges to a smaller error within a higher number of iterations. When $\mu$ exceeds a critical value, the algorithm becomes unstable and diverges producing a huge error.

\subsection{Recommendations}

This thesis sets up a framework for future research in TD stability of free running and hopped oscillators with applications in the development of FH systems under optimal conditions in terms of speed, number of frequencies and processing complexity. The powerful method for obtaining expressions for different variance measures makes possible the design of variance measures, using the "transfer function approach". These measures could even be capable of performing spectral analysis (e.g., Hadarmard Variance). An immediate application of this approach would be to take several of the existing measures (such as the modified Allan and BoileauPicinbono variances) and obtain expressions for them as functions of $p, \tau$, and $T_{1}$, such that the behaviour of these measures can be examined by varying the above 
quantities.

Experimentally, the behaviour of the Barnes-Allan variance could be investigated for different ranges of values of $\tau$. The same can be done for other variance measures as well. This would reduce the observation time and computational complexity required for stability characterization. 


\section{REFERENCES}

[Allan 66]

[Allan 75]

[Analog Devices 80]

[Analog Devices 82]

[Andricos 80]

[Avantek 8i]

[Babitch 74]

[Barnes 66]

[Barnes 71]

[Barnes 86]
D.W.. Allan, "Statistics of Atomic Frequency Standards", Proc. IEEE, vol.54, pp.221-230, February 1966.

D.W. Allan, "Picosecond Time Difference Measurement System", Proc. 29th Annu. Symp. Frequency Contr., pp.404-411, May 1975.

"Analog-Digital Conversion Notes", by the Engineering Staff of Analog Devices, Analog Devices Inc., Norwood, Mass., 1980.

"Data Acquisition Data Book 1982", Volume 1, Analog Devices Inc.

C. Andricos, "An L- and S-band Radar Exciter Using Agile Low Noise Phase Locked Loop Synthesizers", Proc. S4th Annu. Symp. Prequency Contr., pp.202-212, May 1980.

"Solid State Microwave Components Catalog", Avantek Inc., April 1984.

D. Babitch, J. Oliverio, "Phase Noise of Various Cscillators at Very Low Fourier Frequencies", Proc. 28th Annu. Symp. Frequency Contr., pp.150-159, May 1974.

J. Barnes, "Atomic Timekeeping and the Statistics of Precision Signal Generators", Proc. IEEE, vol.54, pp.207-220, February 1966.

J. Barnes et al., "Characterization of Frequency Stability", IEEE Trans. Instrum. Meas., vol.20, pp.105-120, May 1971.

R.L. Barnes, S.H. Ardalan, "Multiprocessor Architecture for Implementing Adaptive Digital Filters", IEEE Int. Conf. in Comm., pp.7.3.17.3.6., 1980 . 
[Barsalou 82]

[Baugh 71]

[Best 84]

[Bisseger 77]

[Bitmead 80]

[Bjerede 76]

[Bloor 80]

[Boileau 76]

[Boileau 78]

B. Barsalou, "A Microprocessor-Controlled VCO as a Spread Spectrum Synthesizer", Master's Thesis, Carleton University, Ottawa, Canada, February 1982.

R. Baugh, "Frequency Modulation Analysis with the Hadamard Variance", Proc. 25th Annu. Frequency Contr., pp.222-225, April 1971.

R. Best, Phase-Lock! Isops: Theory, Design and Applications, McGraw-Hill, New York, 1984.

C.A. Bisseger, "VCO Subsystems: What To Test, How To Test It", Microwaves, vol.16, no.5, pp.60-64, May 1977.

R. Bitmead, B. Anderson, "Performance of Adaptive Estimation Algorithms in Dependent Random Environments", IEEE Trass. on Automatic Control, vol.25, no.4, pp.788-794, August 1980.

B. Bjerede, G. Fisher, "An Efficient Hardware Implementation for High Resolution Frequency Synthesis", Proc. 90th Annu. Symp. Frequency Contr., pp.318-321, May 1976.

R. Bloor, Jr., "MECL System Design Handbook", 3d edition, Motorolz Semiconductor Products Ine., 1980.

E. Boileau, B. Picinbono, "Statistical Study of Phase Fluctuations and Oscillator Stability", IEEE Trans. Instrum. Meas., vol.25, pp.66-75, March 1976.

E. Boileau, "Improvements of the Procedures Used to Study the Fluctuations of Oscillators", IEEE Trans. Instrum. Meas., vol.27, pp.210214, September 1978. 
[BTL Tech.Staff 71]

[Burr-Brown 86]

[Buswell 75]

[Chalmers 79]

[Cooper 86]

[Cravis 63]

[De Haan]

[Dixon 84]

[Dodson 81]

[Drapac 87]

[Egorychev 84]
"Transmission Systems for Communications", by members of the Technical Staff of Bell Telephone Laboratories, Chap.11, Fourth Edition, Bell Telph. Labs., New Jersey, 1971.

"Burr-Brown Integrated Circuits Data Book", Burr-Brown Technical Library, 1986.

Buswell, "VCO's in Modern ECM Systems", Microwave Journal, vol.18, no.5, pp.43-46, May 1975.

E. Chalmers, R. Carman, "Wideband VCO Uses Gun Varactor Tuning", Microwave System News, vol.9, no.1, pp.75-80, January 1979.

G.R. Cooper, Modern Communications and Spread Spectrum, McGraw-Hill, 1986.

H. Cravis, T. Crater, "Engineering of T1 Carrier System Repeatered Line", Bell Systems Tech. Jourmal, vol.42, no.3, pp.431-486, March 1963.

B. De Haan, Nouvelles Tables, New York, Hafner.

R. Dixon, "Spread Spectrum Systems", 2nd Edition, New York, J. Wiley, 1984.

D.J. Dodson, et al., "Advanced SAW-LSI Frequency Synthesizer", Proc. 85th Anru. Symp. Frequency Contr., pp.436-439, May 1981.

M.J. Drapac, "Advances in ESM Receiver Technology", Journal of Electronic Defense, pp.83-87, April 1987.

G.P. Egorychev, "Integral Representation and the Computation of Combinatorial Sums", Amer. Math. Society, vol.59, 1984. 
[Eleftheriou 85]

[Falconer 78]

[Falconer 80]

[Freq. Sources 79]

[Gardner 79]

[Gerber 85]

[Gould 72]

[Harris 84]

[Haykin 84]

[Howe 81]

[Iosifescu 69]
E. Eleftheriou, "Adaptive Filtering in a TimeVarying Environment With Applications to Equalization of Fading HF Channels", Ph.D. Dissertation, Carleton University, Decemberr 1985.

D.D. Falconer, L. Ljung, "Application of Fast Kalman Estimation to Adaptive Equalization", IEEE Trans. on Comm., October 1978, pp.14391446.

D.D. Falconer, V.B. Lawrence, S.K. Tewksbury, "Processor-Hardware Considerations for Adaptive Digital Eilter Algorithms", IEEE International Conf. on Comm., pp.57.5.1-57.5.6, 1980.

"What You Need to Know About Microwave VCO's", Frequency Sources Inc. App. Note, 1979.

F. Gardner, Phase Lock Techriques, 2nd Edition, New York, J. Wiley, 1979.

E. Gerber, A. Ballato, Precision Frequency Control Volume 2: Oscillators and Standards, Academic Press, Orlando, Florida, 1985.

H.W. Gould, Combinatorial Identities, revised edition, Morgantown, W. Va., 1972.

"Analog and Telecommunications Product Data Book", Harris Corporation, 1984.

S. Haykin, Introduction to Adaptive Filters, McMillan, New York, 1984.

D.A. Howe, D.W. Allan, J.A. Barnes, "Properties of Signal Sources and Measurement Methods", Proc. 35th Annu. Symp. Frequency Contr., pp.A1-A41, May 1981.

M. Iosifescu, R. Theodorescu, Random Processes and Learning, Springer-Verlag, New York, 1969. 
[Kalivas 87]

[Kalivas 88]

[Kalivas 90]

[Kalman 61]

[Kandpal 88]

[Kartaschoff 78]

[Lindsey 76]

[Lindsey 78]

[Lindsey 81]

[Manassewitch 76]
G. Kalivas, R. Harrison, "Frequency Stability Characterization of Hopping Sources", Proc. 41st Annu. Symp. Frequency Control, pp.122125, May 1987.

G. Kalivas, R. Harrison, "A Novel Approach to Deriving Closed Form Expressions for Frequency Stability Variance Measures", IEEE Trans. Instrum. Meas., vol.37, pp.231-239, June 1988.

G. Kalivas, R. Harrison, "Characterization of the Frequency Stability of Frequency-Hopping Sources", revised copy submitted for publication to IEEE Trans. Ultras. Ferr., Freq. Control.

R.E. Kalman, R.S. Bucy, "New Results in Linear Filtering and Prediction Theory", J. Basic Eng., Trans. ASME, vol.95, pp.107, 1961.

P.C. Kandpal, "A Phase-Locked 4-6 GHz Local Oscillator Using Microwave Prescaler", Proc. 42nd Annu. Symp. Frequency Contr., pp.364368, May 1988.

P. Kartaschoff, "Frequency and Time", Academic Press, London, 1978.

W.C. Lindsey, C.M. Chie, "Theory of Oscillator Instability Based Upon Structure Functions", Proc. IEEE, vol.64, pp.1652-1666, December 1976.

W.C. Lindsey, C.M. Chie, "Acquisition Behavior of a First-Order Digital Phase-Locked Loop", IEEE Trans. Comm., vol.26, no.9, pp.1364-1370, September 1978.

W. Lindsey, C.M. Chie, "A Survey of Digital Phase-Locked Loops", Proc. IEEE, vol.69, pp.410-431, April 1981.

V. Manassewitsch, "Frequency Synthesizers, Theory and Design", J. Wiley, New York, 1976. 
Mangulis 65]

[Mohr 67]

[Nahi 69]

[Papoulis 65]

[Peregrino 76]

[Phillips 78]

[Raines 86]

[Rb Jdes 80]

[Rutman 74]
V. Mangulis, Handbook of Series for Scientists and Engineers, New York, Academic, 1965.

R. Mohr, "Coupling Between Open and Shielded Wire Lines Over a Ground Plane", IEEE Trans. Electromagnetic Compatibililty, vol.9, no.2, pp.34-45, September 1967.

N. Nahi, Estimation Theory and Applications, J. Wiley, New York, 1969.

A. Papoulis, Probability, Random Variables and Stochastic Processes, McGraw-Hill, 1st edition, New York, 1965.

L. Peregrino, D.W. Ricci, "Phase Noise Measurement Using a High Resolution Counter with Online Data Processing", Proc. S0th Annu. Symp. Frequency Contr., pp.309-317, June 1976.

D. Phillips, "HF Frequency Synthesizer Adaptive to Various Tuning Time and Frequency Increment Requirements", Proc. S2th Arnu. Symp. Frequency Contr., pp.373-377, May 1978.

R.M. Raines, S.A. Blankenship, "The Impact of Advanced Modulation Techniques on EW", Defense Electronics, pp.81-98, October 1986.

R. Rhodes, et al., "Frequency $A_{3}$ 'le PhaseLocked Loop Synthesizer for a $P$ mmmunication Satellite." IEEE National Telecom. Conf., pp.22.3.1-22.3.6, 1980 .

J. Rutman, "Characterization of Frequency Stability: A Transfer Function Approach and Its Application to Measurement Via Filtering of Phase Noise", IEEE Trans. Irstrum. Meas., vol.23, pp.40-48, March 1974. 
[Rutman 78]

[Schoniger 77]

[Stanford Telecomm. 89]

[Stein 88]

[Sutaria 82]

[Texas Instruments 81]

[Watkins-Johnson 80]

[Watkins-Johnson 82]

[Widirow 76]

[Widrow 84]
J. Rutman, "Characterization of Phase and Frequency Instabilities in Precision Frequency Sources: Fifteen Years of Progress", Proc. IEEE, vol.66, pp.1048-1075, September 1978.

H. Schoniger, "Evaluating Tuning Response of VCO Subsystems", Microwave Systems News, vol.7, no.7, pp.45-54, July 1977.

“60 MHz CMOS 32-Bit Modulated NCO STEL1175", Stanford Telecommunications Inc., 1989.

S.R. Stein, R.L. Filler, "Kalman Filter Analysis for Real Time Applications of Clocks and Oscillators", Proc. 42nd Annu. Symp. Frequency Contr., pp.447-452, May 1988.

F. Sutaria, D. Vazirani, "Modern Techniques of FLL Tuning", IEEE Trans. on Consumer Electronics, Vol.CE-28, No.4, pp.536-540, November 1982.

"The TTL Databook for Design Engineers", 2nd edition, by the Engineering Staff of Texas Instruments Inc., Semiconductor Group, 1981.

"Hyperabrupt Varactor Tuned Oscillators", Watkins-Johnson Company Technical Note, 1980.

"Thin-Film Oscillators", Watkins-Johnson Com pany Catalog, 1982.

B. Wirirow et al., "Stationary and Nonstationary Learning Characteristics of the LMS Adaptive Filter", IEEE Proceedings, vol.64, no.8, pp.11511162, August 1976.

i. Widrow, E. Walach, "On the Statistical Efticiency of the LMS Algorithm with Nonstationany Inputs", IEEE Trans. Information Theory, vol.30, no.2, pp.211-221, March 1984. 
[Widrow 85]

[Wiley 82]

[Wolfson 89]
B. Widrow, Adaptive Signal Processing, Prentice-Hall, New Jersey, 1985.

R. Wiley, Electronics Intelligence: The Analysis of Radar Signals, Artech House, 1982.

H.M. Wolfson, "A Very Small Frequency Generator System for Spread Spectrum EHF Applications", IEEE Proceedings in Military Comm., pp.40.6.1-40.6.5, 1989 . 

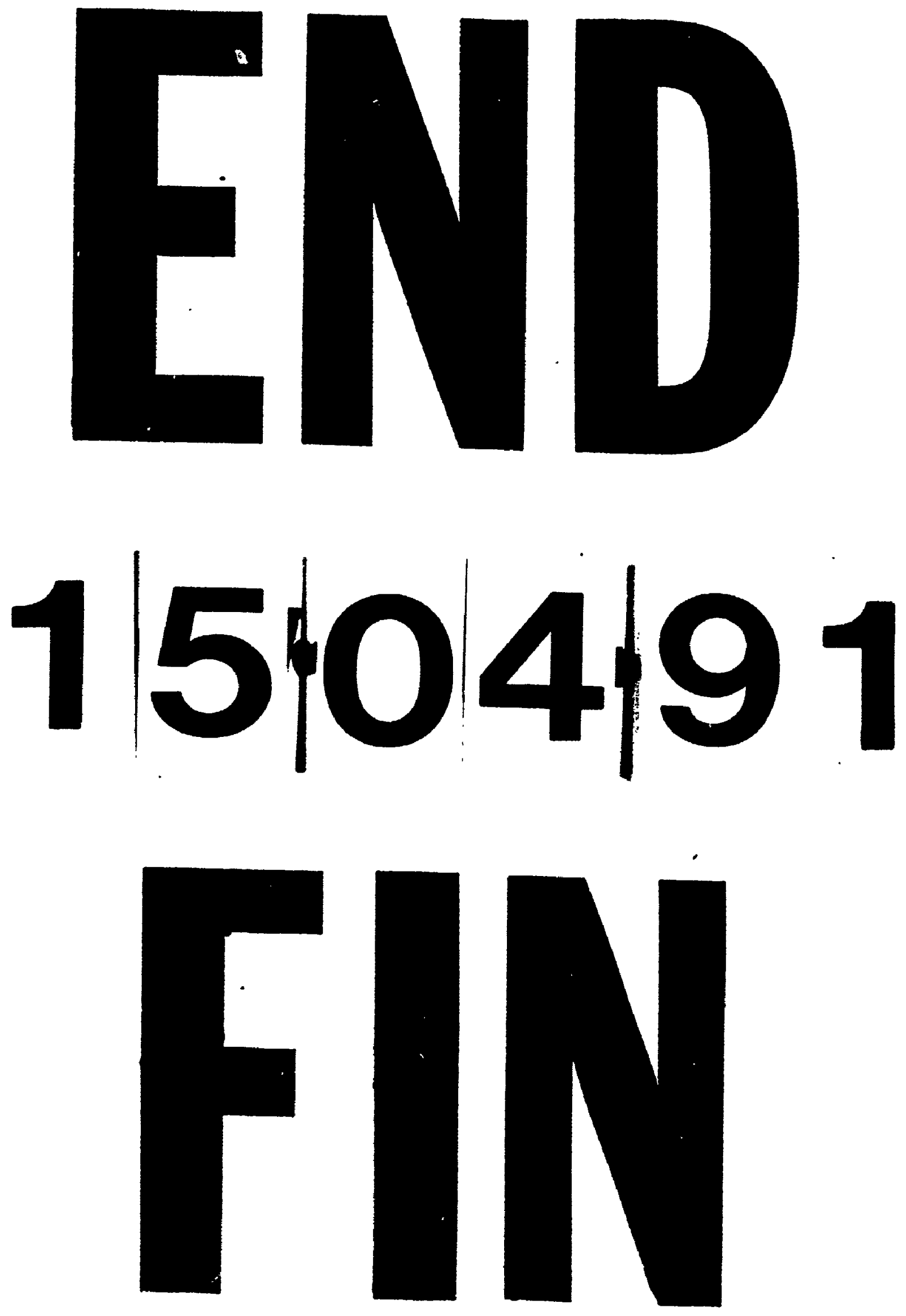